
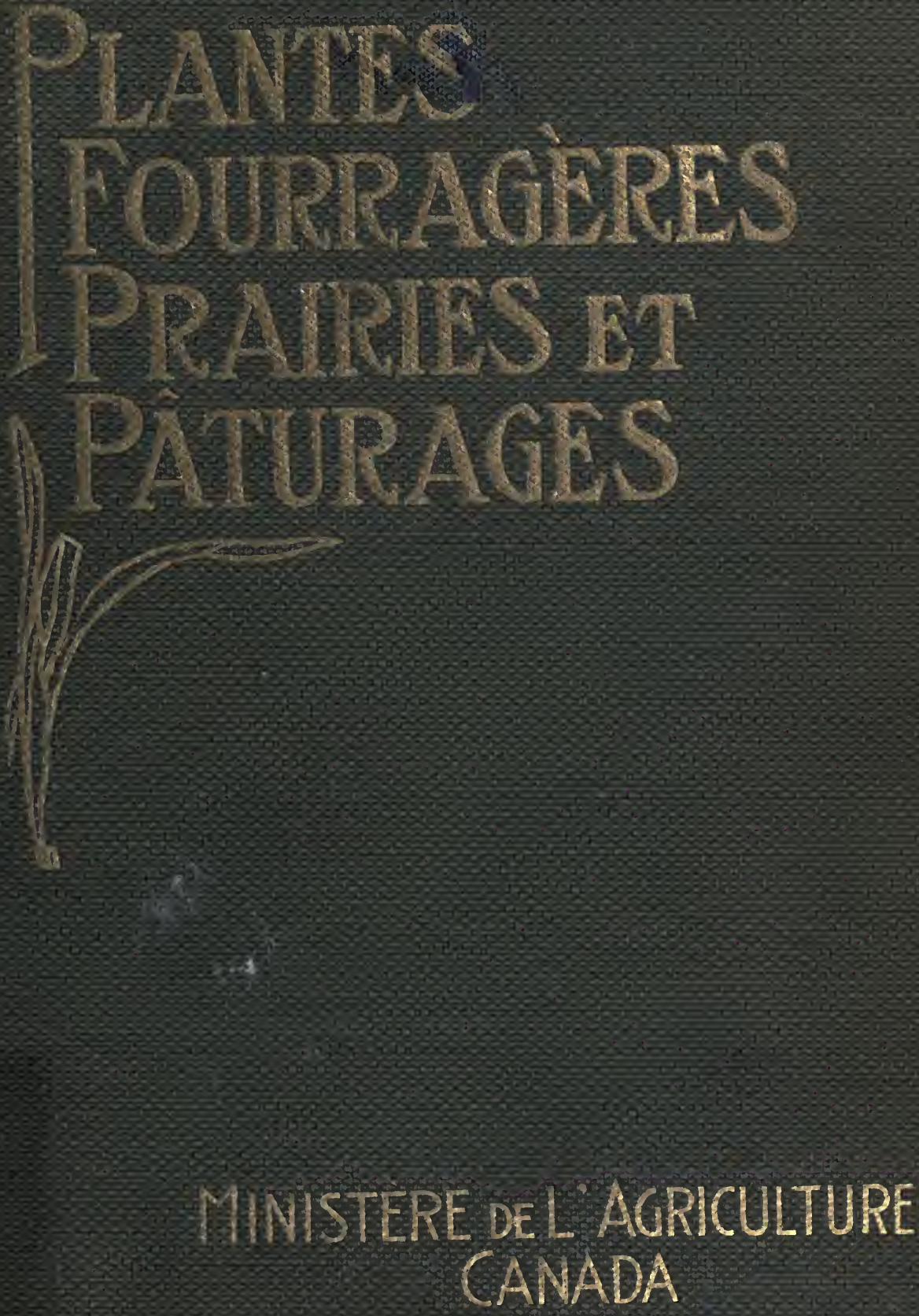


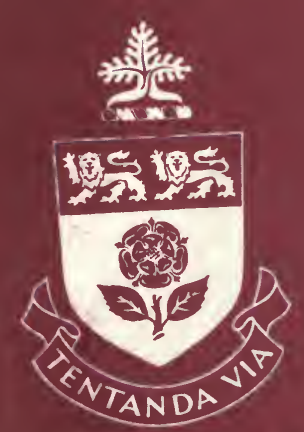

THE LIBRARY OF

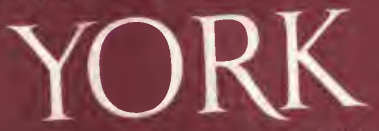
UNIVERSITY 


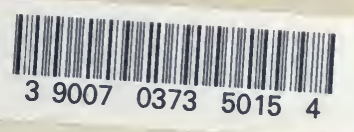





\title{
Plantes Fourragères Prairies et Pâturages
}

\author{
PAR \\ GEO. H. CLARK, B. S. A. \\ ET \\ M. OSGAR MALTE, Ph. D.
}

AVEC GRAVURES A L'AQUARELLE DE NORMAN GRIDDLE

Traduit au Bureau de Traduction du Ministère.

Publié par ordre de
l'Honorable Martin Burreli., Ministre de l'Agricultúre.
Ottawa, i9i3.

En vente au Bureau du Surintendant de la Papeterie, IMPRIMERIE DU GoUvernement, OtTAWA.

Prix, 50 Cents.

$28550-1$ 
SB

208
63
15314 
Trète incarnat. . ...................................... PAGE

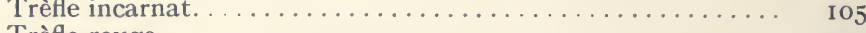

Trèfle rouge.............................. 107

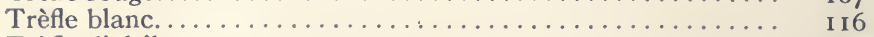

Trèfle d'alsike............................. I 9

Trèfle d'odeur............................... 123

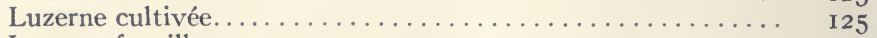

Luzerne faucille........................... I33

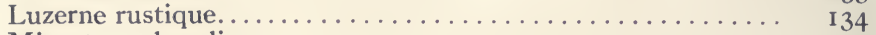

Minette ou lupuline....................... 137

Anthyllide vulnéraire........................ I 39

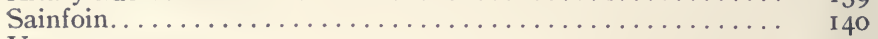

Vesce commune........................ I 42

Vesce velue. .......................... I 44

Fève à cheval........................... I 45

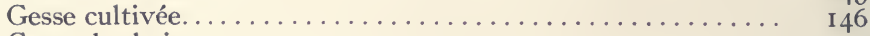

Gesse des bois.......................... I 47

Soja ou Soya............................. I 49

Navette. ......................... I 50

Glossaire........................... I 53

Index...................... 157 


\section{PRÉFACE.}

L'agriculture enrichit ceux qui la connaissent, tandis qu'elle laisse vivre dans la détresse le cultivateur ignorant, quelque peine qu'il se donne.--Xénophon, $l$ 'Economique, 434-355, A.C.

Les cultures fourragères, prairies et pâturages, occupent près d'un tiers de la superficie cultivée au Canada, d'où l'urgence de répandre des renseignements sur les plantes qui entrent dans leur composition. Du reste les nombreuses questions qui parviennent au Ministère fédéral de l'agriculture, surtout depuis quelques années, témoignent éloquemment de l'intérêt très vif que l'on apporte à ce sujet.

Cet ouvrage a donc pour but de fournir, dans un ordre qui en facilite la recherche, des renseignements assez complets sur les graminées, les trèfles et toutes les autres plantes fourragères dont la valeur est généralement reconnue au Canada. Nous y avons fait entrer également un bon nombre de plantes européennes ou récemment importées d'Europe, encore peu connues mais qui, pour une raison ou pour une autre, peuvent un jour se montrer utiles dans certaines localités sinon dans toute l'étendue de notre pays.

Nous ne présentons dans ce volume que des faits et des procédés bien établis; toutes les idées ou théories nouvelles ou douteuses ont été laissées de côté. Nous nous sommes efforcés d'en faire un résumé de données authentiques et indiscutables qui puisse être utile à tous les cultivateurs et agronomes intéressés au développement des vastes ressources agricoles du Canada.

Mûs par le désir d'être facilement compris de toutes les catégories de lecteurs, nous avons évité autant que possible l'emploi de termes scientifiques. Pour ces quelques termes techniques ou semitechniques qui ont dû être conservés, nous avons ajouté un glossaire donnant leur signification exacte.

Dans l'introduction de ce volume, les principaux groupes de plantes ont été étudiés dans un ordre semi-scientifique. Les plantes elles-mêmes ont été classées d'après le système adopté dans la dernière édition du manuel de botanique de Gray.

Les auteurs désirent exprimer leur reconnaissance à $\mathrm{M}$. Leonard S. Klinck, B.S.A., professeur de culture au Collège Macdonald, 
Québec, qui a bien voulu se charger de la critique du manuscrit, et à M. C. A. Zavitz, B.S.A., professeur de culture au collège d'agriculture de l'Ontario à Guelph, qui nous a communiqué nombre de renseignements utiles sur les plantes fourragères de l'Ontario. Les recommandations éminemment utiles de ces experts bien connus nous ont été du plus grand secours.

La version française de cet ouvrage est l'oeuvre du bureau de traduction du Ministère de l'agriculture, sous la direction de M. C. E. Mortureux, B.S.A.

Sans fourrage pas de bétail; sans bétail pas de fumier; sans fumier pas de récoltes.-Proverbe flamand.

Veux-tu du blé, fais des prés, malheur à qui ne fait pas de prés.-Celui qui a le tiers de ses terres labourables en prés est un bon cultlvateur, le quart n'est pas assez. "De temps à autre mets ta terre en pré; après tu es sûr d'avoir du blé. Ainsi ce n'est pas tout de fumer, il faut encore alterner. Alterner, c'est mettre la terre de labour en prairie, puis après un certain temps, remettre la prairie en labour, et toujours de même jusqu'à la fin du monde. C'est le grand secret de la culture; elle est toute là." Jacques Bujault, Euvres, I77I- 1842.

C'est chose convenue d'un chacun que le plus assuré gain est celui qui vient avec le moins de coat. sur laquelle maxime étant fondée, Caton, oracle de son temps, donna cette tant notable réponse: Que pour devenir bien riche, fallait bien paitre; pour devenir moyennement riche, moyennement paltre. Ainsi, à souhaiter le plus du domalne être employé en herbage, trop n'en pouvant avoir pour le blen de la mesnagerie, d'autant que, comme sur un ferme fondement, toute l'agriculture s'appuie 1à-dessus. Aussi voit-on que, moyennant le bétail, tout abonde en un lieu, tant pour le liquide qui, sans attente, en sort, que par les fumiers, causant abondance de toutes sortes de fruits.-Olivier de Serres, Theatre d'agriculture et Mesnage des champs, 1600.

La culture des prairies artificielles est un thermomètre invariable et sûr pour juger l'état de l'agriculture, l'aisance ou la richesse d'un royaume agricole. Une plus grande quantité de fourrages assure la multlpllcation des bestiaux, d'ou résulte nécessairement une masse plus considérable d'engrais qui sont la base essentielle de toute espèce de culture.-Mémoire présenté par la Société royale d'agriculture de France à l'assemblée nationale, le 24 octobre 1789.

Sans les herbages, il n'est pas d'agriculture possible;-avec eux, if en est rarement d'impossible.Maison Rustique du XIXe sizcle. 


\section{Plantes fourragères, prairies et pâturages}

\section{INTRODUCTION.}

I ES débuts de la civilisation sont étroitement liés à ceux de l'agricul-

ture. L'histoire nous apprend que les nations, après avoir longtemps sommeillé dans les ténèbres du barbarisme, dans un passé enveloppé de mythes et de légendes, en sont sorties un jour pour s'avancer lentement vers des idéals plus élevés. Or, toujours ce réveil était associé à un progrès dans la culture du sol. Il est donc exact de dire que l'agriculture est le point de départ des progrès de l'humanité; le degré de perfection qu'elle atteint dans un pays quelconque dépend du climat de ce pays et des facultés naturelles du peuple qui l'habite. C'est principalement à cause des conditions de climat que certaines tribus sont encore nomades et mènent une vie errante, tandis que d'autres se sont établies à demeure. Dans les parties tempérées du globe, où les céréales et autres plantes viennent aisément, l'agriculture est beaucoup plus ancienne que dans les régions reculées vers le nord, moins favorisées au point de vue du climat.

Au début on ne cultivait que ces plantes qui pouvaient servir à la nour riture del'homme; les prairies et les herbages naturels suffisaient aux animaux domestiques. De nos jours même il y a encore de grandes superficies où l'on ne s'exerce aucunement à obtenir des fourrages, mais à mesure que la population devient plus dense, la culture dıs céréales pour l'alimentation de l'homme augmente et la valeur de la terre s'accroît. Les herbages naturels disparaissent graduellement et le cultivateur doit se mettre en quête d'autres plantes pour alimenter ses bestiaux pendant les différentes saisons. C'est dans les régions tempérées, où les animaux ne peuvent paître pendant l'hiver, que la culture des plantes fourragères a atteint son plus grand développement.

Les prairies artificielles sont d'origine beaucoup plus récente que la culture des céréales. Les plus anciennes que l'on connaisse nous viennent des Romains. Les trèfles, qui en forment la partie la plus essentielle, ne sont devenus d'emploi général que vers la fin du seizième siècle. Depuis lors l'importance de ces plantes a été de mieux en mieux comprise.

Deux groupes de plantes sont employés dans la production des fourrages et des herbages, savoir: les graminées et les légumineuses, qui représentent deux grandes familles botaniques désignées respectivement sous ces mêmes nom. Toutes les plantes étudiées dans ce volume, à l'exception de la navette, appartiennent à l'une ou à 
l'autre de ces familles. La navette appartient à la famille de la moutarde, Crucifère.

\section{GRAMINÉES.}

Nom: On comprend souvent parmi les graminées certaines plantes comme les laîches et les carex qui, au point de vue botanique, n'ont aucune des caractéristiques des vraies graminées. D'autre part, beaucoup de gens ne considèrent pas que le maïs et le millet sont de vraies graminées. En agriculture, on fait une distinction entre les céréales et les graminées mais en botanique cette distinction ne saurait exister: le seigle, l'orge, l'avoine et le blé sont des graminées tout aussi bien que la fétuque des prés, l'agrostide et le mil (fléole des prés).

Graine: Si nous coupons avec un couteau tranchant un grain de blé d'Inde (maïs) parallèllement à sa surface la plus large, nous

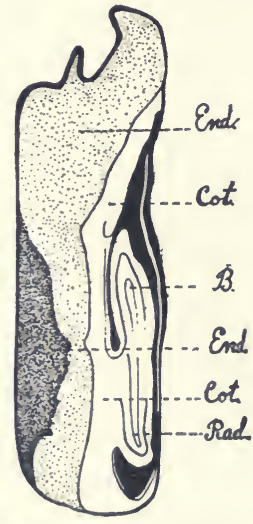

Fig. 1. Grain de mals fendu par le milieu.

Quatre fois la grosseur naturelle.

End.-Endosperme.

Cot.-Cotylodon.

B.-Bourgeon.

Rad.-Radicule. trouvons qu'une grande partie de ce grain se compose d'une masse blanche ou jaune, ne contenant aucune structure visible à l'œil nu. La partie du grain qui, dans la figure I, est marquée "End" est appelée endosperme ou albumen; elle fournit de la nourriture à la jeune plante. Le reste du grain est de couleur terne, on y distingue trois sections à l'œil nu. C'est l'embryon ou la jeune plante avant la germination. Il se compose du cotylédon (fig. I Cot.) qui est situé près de l'endosperme, d'un bourgeon ou "gemmule" (fig. I. B.) d'où naissent la tige et les feuilles de la plante en germination, et d'une radicule (fig. I Rad.) d'où part la première racine. La partie située entre la radicule et la gemmule est la tige de l'embryon.

Germination: Lorsque le blé d'Inde germe, le cotylédon fait l'office de suçoir, il passe à l'embryon la nourriture qui se trouve dans l'endosperme; il reste caché dans le grain pendant la germination. 
Les autres parties de l'embryon deviennent bientôt visibles, la radicule se transforme en racine et la gemmule étale bientôt quelques feuilles vertes. La racine primaire meurt peu après; elle est remplacée par des racines secondaires qui naissent des parties basses de la tige. Cette description représente, dans ses lignes principales, la germination de toutes les graminées.

Racine: La plupart des plantes de prairies ou d'herbages sont vivaces, c'est-à-dire leurs parties souterraines survivent d'une année à l'autre. Ces parties qui survivent se composent de tiges souterraines d'où naissent des racines et des tiges aériennes. Parfois ces tiges souterraines courent dans le sol, avec de longs entre-nœuds, alors les tiges aériennes paraissent clairsemées et toute la plante forme un tapis plus ou moins étendu, comme dans le cas de la fétuque rouge. Dans d'autres cas les entre-nœuds sont très courts, les tiges aériennes sont alors très près l'une de l'autre et la plante pousse en touffes, comme la fétuque ovine. Ce type est spécial à certaines espèces, mais il peut être modifié par les conditions du sol. Par exemple, un sol ferme et compact s'oppose au développement des tiges souterraines et la plante pousse plus ou moins en touffes. D'autre part, les plantes qui ont l'habitude de pousser en touffes forment des touffes plus ouvertes dans les sols légers et peu compacts que dans les argiles lourdes.

Tige: Les tiges des graminées, généralement appelées chaumes, sont creuses, à l'exception de celles du blé d'Inde qui sont pleines, mais elles sont bouchées, à intervalles, par des parties renflées, de diverses couleurs, que l'on appelle nœuds ou jointures. La partie de la tige comprise entre deux nœuds est appelée entre-nœud. Immédiatement au-dessus du nœud une petite partie de la tige reste molle et continue à pousser pendant presque toute l'existence de la plante, mais la partie supérieure de l'entre-nœud durcit rapidement et cesse de croître. Cette disposition permet aux tiges encore jeunes de se redresser lorsqu'elles ont versé sous l'effet des pluies et des vents. 
Feuilles: Les feuilles se composent de deux parties distinctes. La plus basse entoure les tiges comme un étui, généralement ouvert le long d'un côté; c'est la 'gaine'. La partie supé-

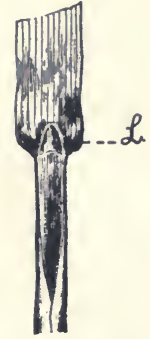

Fig. 2.-Gaine ou partie inférieure d'une feuille de mil.

Grosseur naturelle.

L.-Ligule. rieure, appelée 'limbe' est généralement longue et étroite. Quand les plantes ont une quantité suffisante d'humidité, les limbes sont plats; pendant une sécheresse ils sont souvent enroulés, hérissés, et tournent le dessus vers l'extérieur. La plante peut donc avoir des feuilles roulées pendant une sécheresse excessive et des feuilles plates lorsque l'humidité redevient abondante dans l'air ou le sol. Comme l'humidité obtenue par les racines s'évapore principalement par la surface inférieure des feuilles, cet enroulement des feuilles pendant une sécheresse a pour but de prévenir la perte d'humidité et d'empêcher la plante de mourir de soif. Lorsque le limbe est attaché à la gaine, il y a généralement un appenendice mince, membraneux, de forme et de dimension variables, appelé 'ligule' (fig. 2 L.).

Inflorescence: Les fleurs sont disposées en inflorescences d'aspect souvent très différent, mais toutes construites sur le même principe. L'inflorescence du pâturin bleu de Kentucky est typique (Planche ro). Elle se compose de branches arrangées en verticilles aux nœuds supérieurs de la tige principale. Lorsque les branches sont allongées, comme dans les pâturins, les agrostides, les fétuques, l'avoine, etc., l'inflorescence s'appelle panicule; lorsqu'elles sont très courtes comme dans les millets, vulpins, l'inflorescence a l'apparence d'un épi. Les inflorescences du mil (fléole des prés) (planche 3) et du vulpin des prés (planche 4 ) ressemblent étroitement à des épis réguliers, mais même dans ceux-ci le type est celui de la panicule ordinaire. Une preuve à l'appui de ce fait est que l'on rencontre parfois des inflorescences branchues dans le mil lui-même. Un épi de blé d'Inde est une modification d'une panicule, caractérisée par des branches extrêmement courtes, naissant d'une tige principale charnue.

Dans beaucoup d'herbes, la panicule présente des formes différentes aux diverses phases de son développement. Ainsi, dans l'agrostide et la fétuque ovine les branches sont étalées pendant la floraison et l'inflorescence est alors large et lâche. Lorsque les fleurs sont tombées, les branches se replient vers la tige principale et l'inflorescence devient resserrée et étroite. 


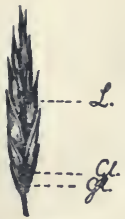

Fig.s. Epilletde brome inerme. Grosseur naturelle.

Gl.-Glumes stériles.

L.-Lemma.

Epillets: Les branches de l'inflorescence portent des épillets aux extrémités; c'est une sorte d'inflorescence partielle (fig. 3). A la base de l'épillet se trouvent deux glumes stériles (fig. 3 G1.) mais le raygrass italien et le ray-grass vivace n'en ont qu'une. Par-dessus ces glumes se trouvent un certain nombre de glumes fertiles appelés lemmas (fig. 3 L.) qui portent une fleur dans l'axe. Chaque fleur est entourée d'une glume délicate appelée palea (fig. $4 \mathrm{~Pa}$.) et se compose principalement de trois étamines (fig. 4 St.) et d'un pistil avec deux stigmates branchus et plumeux (fig. 4. p.).

Le nombre de fleurs varie dans les différentes graminées. Le brome inerme en a de 7 à 9 par épillet, tandis que l'agrostide n'en a qu'une. Dans cette dernière l'épillet entier se compose de deux glumes stériles (fig. 3. G1.), le lemma (fig. 3 L.) et le palea (fig. 4. Pa.) qui enveloppent la fleur proprement dite.

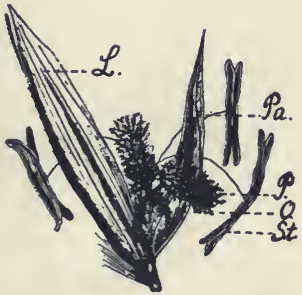

Fig. 4. Fleur d'avoine élevée entourée de ses glumes. Quatre fois la grosseur naturelle. L.-Lemma. P.-Stigmates. Pa.-Palea. O.-Ovaire.

St.-Etamine.

Fécondation: Avant la floraison,les glumes entourent étroitement les fleurs et l'on ne voit rien des étamines ni du pistil. A la floraison les glumes s'ouvrent généralement toutes grandes et l'on voit les étamines et le pistil (fig. 4). Les étamines produisent bientôt des masses poussiéreuses que le vent emporte. Cette poussière est le pollen qui, retenu par les branches des stigmates, féconde l'ovaire c'est-à-dire la partie basse du pistil, (fig. 4 , O.) et un fruit se forme.

Dans le blé, l'avoine et l'orge, le pollen est généralement transporté aux stigmates avant que les glumes de l'épillet commencent de se séparer; chaque fleur est donc fécondée par son propre pollen. Il n'en est jamais ainsi chez les graminées dont nous parlons dans ce volume. Les étamines ne sont prêtes à répandre le pollen qu'après que les glumes se sont ouvertes, et ainsi le pistil est toujours exposé à être fécondé par le pollen d'une autre fleur. En outre, dans beaucoup de graminées, les étamines et le pistil d'une même fleur ne mûrissent pas ensemble et cette circonstance favorise encore cette fécondation croisée. 
Fruit: Après avoir été fécondé, l'ovaire des graminées se développe en un fruit qui renferme une seule graine. A proprement parler, les graines de blé d'Inde, de blé et de seigle sont des fruits qui contiennent une semence, tout comme la noisette est un fruit qui renferme une semence. En réalité la graine de mil, (fléole des prés) dépouillée de sa balle, est un fruit qui renferme une seule semence. Dans la plupart des graminées le fruit reste enveloppé des glumes, et le tout est appelé la graine. C'est ce qui se passe par exemple chez les ray-grass, les fétuques, les pâturins, les agrostides, le mil non décortiqué, etc., dọt la semence, à proprement parler, est un fruit enveloppé de balles ou glumes. Comme cette expression 'graine' est généralement employée, nous nous en sommes servis dans cette description des graminées pour désigner le fruit enveloppé de ses glumes, comme on le trouve généralement dans le commerce.

Valeur agricole: Toutes les herbes sauvages, ou à peu près toutes, peuvent servir de nourriture au bétail à une phase ou à une autre de leur existence. Même les herbes des déserts ou des localités inhospitalières, qui sont sèches, ligneuses, peu savoureuses pendant la plus grande partie de l'année, peuvent fournir une nourriture nullement à dédaigner lorsqu'elles sont jeunes ou rafraîchies par la pluie. Toutefois, les herbes sauvages ont généralement beaucoup moins de valeur que les herbes cultivées. Ces dernières sont mieux soignées, elles ont plus de nourriture à leur disposition, la lutte pour la vie leur est moins pénible, elles peuvent donc faire une pousse plus énergique et rendre une meilleure qualité de foin ou de fourrage.

Lorsqu'on se propose de cultiver une herbe sauvage ou même une variété cultivée, il faut tout d'abord s'assurer si le climat et le sol lui conviennent et si elle répond au but pour lequel on la cultive. Différentes herbes peuvent avoir différentes exigences. Toutes, bien entendu, exigent une provision suffisante d'eau et de nourriture, mais ce qui suffit à une pourrait ne pas empêcher une autre de mourir de faim. Une quantité d'eau qui provoquerait une végétation luxuriante d'une certaine graminée pourrait nuire à une autre espèce, peut-être proche parente de la première. Par exemple, la fétuque ovine vit à l'aise là ou la fétuque des prés souffrirait beaucoup. D'autre part, le dactyle pelotonné languirait dans un terrain frais et acide où la calamagrostide, le vulpin des prés et le pâturin des marais pousseraient avec vigueur. Il importe donc de choisir des variétés adaptées à la localité.

Certaines herbes comme l'agrostide qui ont des racines rampantes et poussent à partir du début même du printemps jusqu'à la fin de 
l'automne, lorsque la température est favorable, conviennent tout particulièrement pour les pâturages car elles résistent au piétinement et fournissent une nourriture verte toute la saison. D'autre part elles ne valent pas grand chose pour faire du foin car la plupart des feuilles sont assez près de terre. Le mil (fléole des prés) et les herbes de ce genre sont moins bonnes pour les pacages, car leur croissance en touffes et leurs racines traçantes les exposent à être déracinées ou du moins à souffrir du piétinement, mais ce type de graminée fournit un foin excellent.

Le développement et la durée d'une herbe sont également des facteurs importants. Il en est qui se mettent à pousser très tôt au printemps et qui sont précieuses lorsqu'on a besoin de foin ou de pâturage hâtifs. D'autres commencent tard, poussent assez lentement et conviennent pour la production de foin ou de pâturages tardifs. Quelques herbes durent peu et meurent après la première ou la deuxième année: le ray-grass italien, par exemple, peut servir dans un assolement de courte durée, mais il ne vaut rien pour un pâturage permanent. La plupart des herbes vivaces atteignent leur complet développement la deuxième ou la troisième année après avoir été semées et sont utiles lorsqu'on désire établir une prairie ou un herbage permanent.

Il est rare que l'on cultive une variété seule, à moins que ce soit pour la production de la graine, car les mélanges de graminées ou de graminées et de trèfles donnent généralement un rendement plus élevé et de meilleure qualité. Par exemple le dactyle pelotonné est généralement cultivé avec d'autres variétés; cultivé seul, il fournirait un fourrage grossier, peu digestif et peu savoureux. Pour obtenir le plus gros rendement possible de bon fourrage, on emploie donc des mélanges qui donnent la plus grande quantité possible de foin ou d'herbe de la plus haute valeur nutritive.

Pour obtenir un fort rendement, il ne suffit pas de choisir ces herbes qui produisent beaucoup lorsqu'elles sont cultivées seules; il faut encore qu'elles soient adaptées au sol et au climat, qu'elles s'accordent ensemble et qu'elles utilisent au mieux possible chaque pouce de terrain. Quand on désire avoir du foin, la valeur du mélange ne dépend pas seulement de la valeur des herbes prises séparément mais aussi du fait qu'elles mûrissent ensemble. Un mélange idéal se compose d'espèces qui arrivent à floraison en même temps. Pour le foin, on fauche généralement au début de la floraison. Si l'on cultivait ensemble des espèces très hâtives et très tardives, le rendement serait relativement faible et la qualité du foin infér eure. Le sol, la chute de pluie et d'autres facteurs déterminent les sortes que l'on doit employer. 
On cultive souvent les trèfles avec les graminées; ce mélange donne une nourriture mieux équilibrée et n'appauvrit pas autant le sol que les graminées seules, qui exigent beaucoup d'engrais. Une tonne de foin de mil contient environ dix-huit livres d'azote, six livres et demie d'acide phosphorique et vingt-huit à trente livres de potasse; c'est là un peu plus que n'apporte à la terre une tonne de fumier de ferme frais ordinaire. Si l'on n'applique pas d'engrais chimiques, il est évident que la culture continue $\mathrm{du}$ mil épuisera rapidement le sol; il en est de même, d'une façon générale, des autres graminées. Les plantes légumineuses (voir page 20) sont très précieuses parce qu'elles accumulent l'azote de l'air et améliorent le sol. Les trèfles apportent de l'azote au sol et maintiennent ainsi une partie de sa fertilité.

\section{PLANTES LÉGUMINEUSES.}

Ces plantes appartiennent à une nombreuse famille, d'un type distinct, appelée Leguminosa. Elle comprend les pois, les vesces, les fèves, le trèfle rouge, le trèfle d'alsike et la luzerne, c'est-à-dire les plantes que le cultivateur appelle communément des légumineuses et des trèfles. Dans son acception habituelle, ce terme comprend les trèfle rouge, trèfle d'alsike, trèfle blanc, trèfle incarnat, luzerne, minette, mélilot et autres plantes légumineuses. Mais au point de vue botanique, les quatre premiers seulement sont des trèfles, au vrai sens du mot, c'est-à-dire ils appartiennent au genre Trifolium tandis que la minette, la luzerne et le mélilot appartiennent à un autre genre.

Graine: Quand on fend une fève ou un pois, on constate que les deux moitiés semblent n'être retenues ensemble que par l'enve-

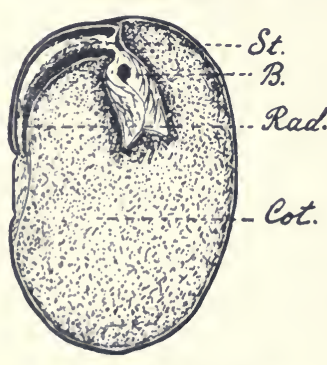

Pio 6. Feve fendue.

Quatre fois la grosseur naturelle. B.-Bourgeon. Rad.-Radicule. $\begin{array}{ll}\text { B.-Bourgeon. Rad.-Radicule. } \\ \text { St.-Tige. } & \text { Cos.-Cotylédon. }\end{array}$ loppe de la graine. L'une d'elle (fig. 5) a une surface lisse, plus ou moins luisante, sur laquelle aucune texture spéciale ne se révèle à l'œil nu. L'autre moitié porte, près de son extrémité supérieure, un organe singulier, composé de deux parties distinctes. La partie de dessus est un bourgeon (fig. 5 B) qui correspond à une formation semblable dans l'embryon des graminées (voir page 8). La partie de dessous, qui est en contact avec l'enveloppe de la graine, est épaissie à un bout (fig. 5 St.) et effilée, à l'autre bout (fig. 5 Rad.) 
Le bout épais est la tige de l'embryon, le bout effilé sa racine ou radicule. Les deux cotylédons de l'embryon remplissent, à eux deux, presque toute la semence. Un embryon de légumineuses a donc deux cotylédons tandis qu'un embryon de graminées n'en a qu'un. Mais une légumineuse n'a pas d'endosperme. La fonction de l'endosperme d'une graine de graminée, comme nous l'avons dit à la page 8 , est de fournir de la nourriture à l'embryon pendant la germination. Dans une plante légumineuse cette fonction est remplie par les deux cotylédons qui sont épais et remplis de nourriture.

Germination: Lorsque la graine d'une plante légumineuse germe, le bourgeon (Fig. 5, B.) se transforme en tige et en feuilles tandis que la radicule (Fig. 5, Rad.) donne naissance à la racine de la plante. La tige de l'embryon (Fig. 5, St.) se comporte de façon différente dans différentes plantes. Dans les haricots, elle se développe en longueur et soulève avec elle, au-dessus du sol, les cotylédons (Fig. 5, Cot.) qui s'aplatissent et s'amincissent graduellement. Dans les pois la tige est courte et les cotylédons restent longtemps cachés dans le sol, entourés de l'enveloppe de la semence.

Racine: Les légumineuses sont annuelles, bisannuelles ou vivaces. Dans les annuelles, comme le trèfle incarnat, ou dans les bisannuelles, comme le mélilot, la racine primaire de l'embryon se développe toujours en une racine pivotante. Dans les légumineuses vivaces, la racine est pivotante ou se compose d'une souche d'où partent des racines secondaires. Ces racines secondaires sont généralement peu profondes et les plantes qui en sont pourvues tirent leur nourriture de la surface. Par contre une racine pivotante descend généralement à une profondeur considérable et la plante tire une bonne partie de sa nourriture du sous-sol. Les racines secondaires, de même que les pivotantes, portent de petits tubercules ou groupes de renflements ou nodosités. Ces tubercules sont importants, nous en reparlerons à la page 20 .

Tiges: Les tiges des plantes légumineuses sont en général dressées ou ascendantes. Parfois, mais rarement, elles sont rampantes et émettent des racines secondaires de leurs noeuds. Les plantes de ce type forment des tapis plus ou moins étendus dans lesquels il est difficile de distinguer les plantes isolées. Il en est souvent ainsi lorsque les tiges partent d'une souche très étendue et très rameuse comme dans la gesse des bois. Dans certaines espèces et certains genres comme dans les pois et les vesces, les tiges sont faibles et 
s'accrochent, pour ne pas tomber à terre, à des organes spéciaux sur les feuilles, appelés vrilles (voir ci-dessous.)

Feuilles: Les feuilles des légumineuses sont composées, c'està-dire chaque feuille se compose d'un certain nombre de petites feuilles ou folioles, entièrement séparées les unes des autres. Nous en trouvons le type dans le sainfoin-une feuille composée d'un certain nombre de paires de folioles et se terminant par une seule foliole (Planche 23). Toutes les autres sortes ne sont que des modifications de ce type. Ainsi quand les folioles ne sont qu'au nombre de trois comme dans le trèfle rouge, la luzerne, le mélilot et autres, on obtient la feuille trifoliée bien connue. Dans d'autres espèces, comme les vesces (planches 24 et 25) et les pois, les limbes des folioles supérieures ne sont pas développés; il ne reste que leurs nervures qui se transforment en vrilles dont la fonction est de soutenir les tiges faibles.

Chacun sait que dans une récolte très épaisse de pois ou de vesces les plantes sont parfois si fermement attachées les unes aux autres qu'en les arrachant au bout d'une longue rangée on fait remuer les plantes à l'autre bout de la rangée. C'est parce que les vrilles s'enroulent autour des tiges et des branches des plantes voisines et les lient ensemble. Ces vrilles sont de merveilleux organes. Frottez-en une doucement avec un brin de paille, et elle répondra en se courbant. Donnez-lui l'occasion de saisir la branche d'une plante voisine et elle s'enroulera si étroitement autour de cette branche qu'il sera impossible de l'en détacher sans la rompre. Elle a le sens du toucher et la faculté d'agir. Sa sensibilité est si developpée que quelques vrilles peuvent sentir un poids d'un quart de milligramme. 


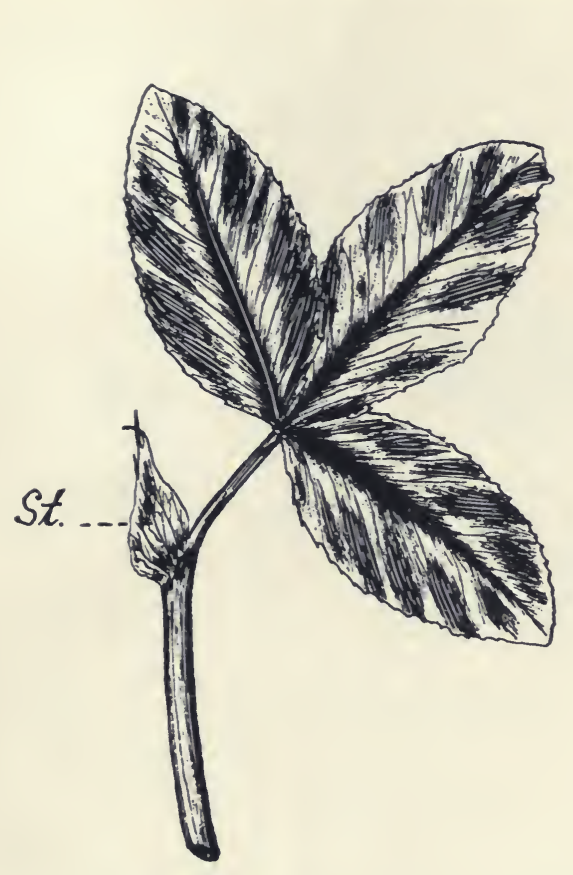

Fig. 6. Feuille de trèfle d'alsike. Grosseur naturelle. St.-Stipule.
Deux appendices, les stipules, sont attachés à la base de la queue de la feuille (Fig. 6, St.) Ils sont généralement étroits, d'aspect insignifiant, mais parfois, par exemple chez les pois, ils ont la même forme que les folioles et sont presque aussi gros que celles-ci.

\section{Inflorescence :}

Les fleurs des légumineuses sont en groupes, d'aspect différent mais toujours construits d'après le même principe. Parfois ils sont longs et recouverts d'un nombre de fleurs relativement restreint comme dans les vesces (planches 24 et 25). C'est ce

que l'on appelle des grappes. Dans d'autres plantes les grappes sont courtes et les fleurs serrées l'une contre l'autre, comme dans les trèfles rouge et d'alsike. Cette inflorescence porte le nom de capitule. Toutefois il est impossible d'établir une distinction bien nette entre un capitule et une grappe. Par exemple, l'inflorescence de la luzerne (pl. $2 \mathrm{I}$ ) et celle du trèfle incarnat (planche $\mathbf{1 7}$ ) ressemblent autant à des grappes courtes qu'à des capitules allongés.

Fleurs: Les fleurs de toutes les légumineuses ont la même structure générale, tout à fait différente des fleurs des autres familles de plantes. La partie inférieure (fig. 7, Sep.) est insignifiante. Elle se 28550-2 
compose de cinq organes verts, en forme de dents, appelés sépales, et reliés à la base. La partie supérieure, appelée communément la fleur, se compose de cinq pétales, la plupart de couleur voyante. L'un de ceux-ci, beaucoup plus gros que les autres, les enveloppe tous dans le bourgeon. C'est l'étendard (fig. 7, S.). Les pétales latérales, de forme irrégulière, sont appelés ailes (fig. 7, W.). Les deux autres poussent ensemble, formant un organe en forme de bateau que l'on.

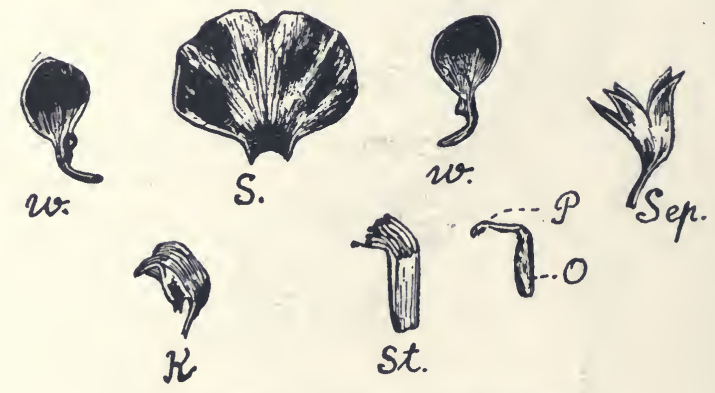

Fio. 7-Les différentes parties d'une fleur de pois.

Grosseur naturelle.

Sep.-Sépales.

S.-Etendard.

$$
\begin{array}{ll}
\text { W.-Ailes. } & \text { St.-Etamines. } \\
K \text {.-Carene. } & \text { O.-Ovaire du pistil. }
\end{array}
$$

$P$.-Stigmate du pistil.

appelle la carène (fig. $7, \mathrm{~K}$.) qui entoure les étamines et le pistil (fig. 7 , St.). Neuf des dix étamines poussent ensemble dans leurs parties inférieures, formant un tube qui enveloppe le pistil. Chaque fleur n'a qu'un pistil, lequel se compose d'une partie inférieure, large, qui est l'ovaire (fig. 7,0 .) et d'une partie supérieure, étroite, brusquement courbée à la base, qui se développe au sommet en un stigmate (fig. 7, P.).

Fécondation: Chez les plantes légumineuses la fécondation n'est jamais provoquée par les vents. Dans certains genres, comme les pois et les vesces, les fleurs se fécondent d'elles-mêmes, c'est-à-dire le pollen féconde automatiquement le pistil de sa propre fleur. Mais dans la plupart des plantes légumineuses le pollen est transporté d'une plante à l'autre par des insectes qui visitent les fleurs à tour de rôle pour en extraire le miel emmagasiné à la base. Dans les fleurs grosses et voyantes, l'étendard attire l'insecte, il lui indique où se trouve le miel. Dans d'autres espèces, les fleurs, assez petites, sont très nom- 
breuses et se voient de très loin. D'autres encore ont des fleurs très peu voyantes, portées près de terre. Certaines plantes comme le lupin noir, poussant à l'ombre d'autres, plus élevées, sont plus ou moins cachées, mais dans ce cas ces fleurs modestes et cachées ont un parfum prononcé qui attire les insectes. On trouvera une brève description de la fécondation, qui varie dans les différents genres, dans l'étude sur la luzerne, page I 25 et sur le trèfle rouge, page I07.

Fruit: Le fruit est une gousse; c'est-à-dire un fruit étroit, à parois de parchemin ou de cuir. Quand elle est mûre et sèche, cette gousse se fend sur toute sa longueur et laisse sortir les semences. Ses deux moitiés se tordent comme un tire-bouchon, parfois avec une telle violence que les graines sont projetées très loin. Dans certaines espèces et certains genres il n'y a qu'une graine, et alors la gousse tombe sans s'ouvrir, mais généralement les graines sont nombreuses.

Valeur agricole: Sur un terrain bien préparé, possédant une bonne quantité de principes fertilisants et surtout de potasse et d'acide phosphorique, les légumineuses donnent de fortes récoltes, très nourrissantes et dont raffolent tous les bestiaux. Sans parler de leur utilité dans la stabulation, les légumineuses peuvent être utilisées avec avantage pour le foin et pour les pâturages. Leur valeur comme fourrage dépend principalement de leur mode de développement. Règle générale, c'est au moment de la floraison ou un peu avant la floraison qu'elles sont le plus nourrissantes. Si l'on veut en faire du foin, il ne faut donc pas les couper trop tard. Il est vrai que parfois on obtient une plus forte récolte en ne fauchant qu'après la floraison, mais alors le foin est grossier et plus ou moins ligneux. Il perd de la succulence et les éléments qui forment le gras et le lait s'y trouvent en moins grande proportion. On a donc plus de foin mais moins de nourriture que si la plante avait été fauchée au bon moment. Enfin une coupe tardive nuit également au regain. Par exemple, quand le trèfle rouge et la luzerne commencent à fleurir, de nouveaux rejetons naissent au collet de la racine; si l'on retarde la coupe jusqu'à ce que ces rejetons soient assez développés pour être rognés par la faucheuse, il est évident que la deuxième pousse en souffrira beaucoup.

Certaines espèces, comme le trèfle blanc, se trouvent bien du piétinement du bétail, qui provoque chez elles une nouvelle végétation. Elles conviennent donc pour les pâturages. D'autres, au contraire, comme le trèfle rouge et la luzerne, dont le collet dépasse 
un peu le niveau du sol, souffrent du piétinement, surtout lorsque le sol n'est pas en bon état. Les pâturages de ces plantes exigent des soins particuliers. Comme la nouvelle pousse part du collet, il ne faut pas faire pâturer les plantes trop au ras de terre ni trop tard à l'automne.

On sait que les légumineuses enrichissent le sol; on attribuait autrefois cette faculté au développement de leurs racines. On prétendait que celles-ci allaient chercher dans les profondeurs du soussol une grande quantité de nourriture inaccessible aux racines plus courtes des autres plantes. On disait que les substances ainsi enlevées au sous-sol étaient utilisées dans le développement des racines de surface et des parties aériennes des plantes, qu'elles revenaient au sol de surface quand on enfouissait les tiges à la charrue. Il est très vrai que les légumineuses enlèvent au sous-sol des principes fertilisants qu'elles emmagasinent dans leurs parties supérieures, et cette explication n'est pas à dédaigner. Mais elle ne suffit pas car on observe d'autres phénomènes associés à cet enrichissement du sol par les légumineuses. Généralement la luzerne ne prend pas sur des sols où elle n'a jamais été cultivée auparavant. Bientôt la végétation s'arrête, les plantes jaunissent et finissent par mourir. Mais si l'on épand sur le sol de la terre prise sur une ancienne luzernière on obtient bientôt et sans aucune peine une récolte. Or, si l'on examine les plantes, on constate que les racines de celles qui poussent sur la vieille luzernière sont munies de nombreuses nodosités ou tubercules tandis que les racines des plantes faibles, sur la terre vierge, en sont dépourvues. Ce n'est que tout récemment que l'on a compris l'origine et l'importance de ces tubercules. Il a été démontré que c'est une sorte de gale, produite par certaines bactéries. Ces bactéries vivent dans le sol, attaquent le chevelu des racines, passent au travers de leurs parois minces et s'introduisent dans l'intérieur. Arrivées là, elles se propagent rapidement et forment ces tubercules dans lesquels elles vivent en colonies. Plus tard, la plupart de ces germes se décomposent et sont utilisés par les plantes qui en tirent un supplément de nourriture. Cette nourriture se compose de substances azotées que les bactéries puisent dans l'air passant par le sol poreux. Ainsi, par l'intermédiaire des bactéries, les plantes légumineuses se procurent leur azote de l'air. Elles l'accumulent, pour leurs propres besoins, sans en demander au sol et, quand elles meurent, en laissent une quantité dans la terre pour les récoltes suivantes.

'La terre d'une bonne luzernière renferme ces germes, et il suffit, pour rendre propre à la culture de la luzerne un champ jusque là 
réfractaire, d'y épandre un peu de cette terre. Il n'en faut pas beaucoup, 200 livres par acre suffisent. On peut aussi, au lieu d'employer de la terre de vieille luzernière, se servir de cultures artificielles de bactéries que l'on peut se procurer à un grand nombre de laboratoires. Ces cultures, avec des instructions pour leur emploi, sont offertes en vente en flacons, à bas prix.

Les bactéries qui forment ces tubercules sont indispensables au développement de toutes les sortes de plantes légumineuses. Mais ceci ne veut pas dire que les bactéries qui servent pour une certaine plante peuvent servir pour une autre. Tout au contraire, il est différentes espèces et différentes races de bactéries et chaque espèce ou chaque race produit des nodosités sur une certaine sorte de légumineuse. Ainsi les bactéries qui opèrent sur les racines de trèfle rouge sont différentes de celles qui produisent des tubercules sur les racines de la luzerne, et ne peuvent faire aucun bien à cette dernière plante. Quand on emploie des cultures artificielles de bactéries il faut donc avoir soin de choisir la sorte convenable.

\section{ÉTABLISSEMENT DES PRAIRIES ET DES PÂTURAGES.}

La préparation du sol, avant l'engazonnement, se fait principalement en vue des exigences de la plante qui doit abriter les herbes et les trèfles. Il faut que le sol de surface soit bien ameubli, bien émietté, bien égoutté, et qu'il soit riche en matières végétales en décomposition. Les jeunes herbes délicates exigent beaucoup d'humidité mais elles souffrent d'un excès de fraîcheur. Si le sol manque d'humus et qu'une crô̂te dure se forme à la surface, la végétation s'arrête et les jeunes plantes souffrent, même si la sécheresse ne devait durer que quelques jours.

Les semis d'herbes devraient être faits après une récolte nettoyante qui a été bien entretenue et bien binée. Avant d'établir une prairie, il faut, tout d'abord, détruire les mauvaises herbes vivaces. Les pentes de collines formées d'un sol argileux, exposé à durcir après de fortes pluies, profitent beaucoup d'une légère application de fumier bien pourri que l'on incorpore au sol à la herse. Les terres basses et humides doivent être égouttées aussi parfaitement que possible, même pour les herbes qui aiment beaucoup l'humidité. Sur les sols de prairie, plus secs, il faut rouler le sous-sol afin de faire monter l'eau près de la surface jusqu'à ce que les jeunes plantes soient devenues assez robustes. 
Plantes-abris: Le but principal des plantes-abris est de protéger la jeune herbe. On doit se servir d'une plante-abri lorsqu'il n'est pas nécessaire de réserver toute l'humidité du sol pour la prairie naissante. Elle protège les jeunes plantes contre la chaleur brûlante du soleil. Elle tient les mauvaises herbes en échec jusqu'à ce que les bonnes aient acquis assez de vigueur pour leur tenir tête. Enfin, elle permet d'obtenir une récolte tandis que la prairie se forme. On considère généralement que le blé ou l'orge font la plante-abri la plus satisfaisante. L'avoine, même semée claire, donne trop d'ombrage et mûrit trop tard. Dans une céréale ensemencée d'herbes on devrait toujours pouvoir voir la jeune herbe, à n'importe quel moment de la végétation, à dix ou douze pieds de l'endroit où l'on se tient. La plante-abri devrait être prête à être récoltée dès que les herbes commencent à taller et que les trèfles ou autres légumineuses commencent à émettre de nouveaux rejetons du collet.

Dans les districts où la chute de pluie n'atteint pas trente pouces de hauteur ou n'est pas bien répartie pendant la saison de végétation, il peut arriver que la plante-abri prive la jeune herbe de l'humidité nécessaire. En certaines saisons il est difficile d'obtenir une bonne levée de trèfle rouge, un peu à cause du manque d'humus dans la terre mais aussi parce que la plante-abri-généralement l'avoineenlève l'humidité dont les jeunes plantes auraient besoin pour se développer. Dans ce cas, si l'on attache plus d'importance à obtenir une prairie qu'une céréale, on fera bien, en saison sèche, de se passer de cette dernière, ou, si elle a été semée, de la couper pour en faire du fourrage avant que l'herbe meure de soif.

La profondeur des semis dépend de la sorte de graine employée, du caractère et de l'état du sol et de l'humidité. On dit que la graine ne devrait pas être semée à une profondeur de plus de quatre fois son diamètre. Les plantes de prairies et d'herbages qui poussent à l'état sauvage laissent tomber leurs graines mûres qui germent à la surface du sol ou très près de la surface. Mais le cultivateur ne saurait être aussi prodigue que la nature; il doit fournir les meilleures conditions possibles pour assurer une végétation parfaite.

Mode de semis: Quand le sol est très ferme, comme lorsqu'on sème au printemps dans un champ de blé d'automne, on peut, si la terre est raisonnablement sèche, semer à la volée puis herser; la terre traitée de cette façon est bien ameublie, la graine d'herbe bien recouverte et le blé en profite également. Quand on sème après ameublissement profond au printemps, on peut répandre la graine 
d'herbe au semoir devant les tuyaux à grain, puis on roule et on passe la désherbeuse (weeder). Si la surface est bien ameublie et la couche au-dessous bien rassise, il suffira, après le semoir, de faire passer la désherbeuse qui aplanira le sol et redistribuera les graines qui auraient pu tomber ensemble entre les rangs. Si la température est favorable on obtient parfois de bons résultats en épandant la graine à la volée, après que la plante-abri a été semée, et en comptant sur la pluie pour la recouvrir et la protéger pendant la germination, mais cette pratique n'est pas à recommander. Il vaut toujours mieux adopter une méthode qui assure une distribution parfaite de la graine et la laisse recouverte d'un demi-pouce de terre. Les graines laissées non recouvertes, dans les sillons et les fossés, sont exposées à être enlevées par une forte pluie. De même, bien des sortes de graines d'herbe qui mettent deux ou trois semaines à germer peuvent être détruites lorsqu'elles sont exposées à la surface. Sur les sols de prairie on recommande d'enterrer les graines de luzerne et des autres plantes fourragères à un pouce ou un pouce et demi de profondeur. Dans les districts semi-arides et quand on cultive la luzerne pour sa graine, il vaut mieux semer clair, en rangs de vingt à trente pouces d'écartement. Si la terre est très sèche, la végétation sera lente, mais les racines profondes de la plante lui permettront de se procurer assez d'humidité pour produire une récolte passable de bonne graine.

Machines: Il existe des instruments spéciaux pour semer les graines d'herbe et de trèfle. La plupart des semoirs à grain sont pourvus d'une boîte qui s'ajuste parfois devant les tuyaux, parfois derrière, et qui sert à semer la graine d'herbe. Sur une surface bien ameublie, où l'on fait suivre le semoir d'une herse légère afin d'aplanir le sol, les graines fines, semées de cette façon ne sont jamais couvertes trop profondément; mais sur une terre argileuse, prise en mottes, elles le sont fréquemment. Le semoir à main, à disques rotatifs pour éparpiller la semence, donne de bons résultats avec la plupart des graines d'herbe et de trèfle; il est généralement employé. Toutefois, quand on sème des mélanges, l'emploi de cet instrument présente les mêmes inconvénients que l'épandage à la main; la graine de trèfle, plus lourde, est projetée beaucoup plus loin que celle des herbes plus fines, et la distribution est inégale.

Les semis épais sont généralement recommandés par les grainetiers et les cultivateurs expérimentés, surtout lorsque la prairie doit être de peu de durée. Règle générale, la récolte n'est pas plus forte que lorsque l'on sème clair, mais la qualité du foin est meilleure. Dans des conditions favorables de sol et de température, on peut 
obtenir un gazon bien fourni en semant un mélange de quatre livres de mil, trois livres de trèfle d'alsike et six livres de trèfle rouge à l'acre. Mais en semant six livres de mil, quatre livres de trèfle d'alsike et dix livres de trèfle rouge, on est plus sûr d'obtenir une récolte serrée, les mauvaises herbes sont moins nombreuses et l'on a une forte récolte de foin de bonne qualité. Le surcroît de dépense occasionné par l'augmentation dans la qualité de graine est, en somme, une assurance peu coûteuse. D'autre part, les semis épais ne sont pas à recommander lorsqu'on cultive une plante fourragère pour sa graine, car une récolte très serrée donne moins de graine, et une graine de moins bonne qualité.

La qualité de la graine est un facteur important dans la formation d'une prairie. Le loyer de la terre, les frais de préparation sont beaucoup plus élevés que le prix de la graine, or si le pourcentage de la graine qui peut germer est faible et, en outre, si cette graine viable n'est pas conforme au type ou si elle est remplie de semences de mauvaises herbes dangereuses, le tout peut se chiffrer par une perte ou, ce qui est encore pire, résulter en un dommage sérieux.

L'origine des graines de graminées et de trèfles est souvent la cause de différences aussi sensibles entre ces graines que celles qui existent entre les variétés, surtout au point de vue de la rusticité. Les graines produites sous un climat chaud donnent des plantes peu résistantes à l'hiver; celles produites sous un climat humide et tempéré sont plus susceptibles à la sécheresse que les plantes parfaitement acclimatées. Des essais de culture de luzerne faits à Guelph ont permis de constater que la graine produite dans le nord, et surtout celle provenant d'anciennes luzernières du district, est plus rustique que la graine provenant de climats plus secs et plus chauds. De même, la graine de trèfle rouge venant du sud de l'Europe ou du Chili ne résiste pas aussi bien à l'hiver canadien que celle produite au pays. Les bons grainetiers devraient connaître l'origine des graines de graminées et de trèfles qu'ils vendent et les acheteurs devraient insister pour avoir des graines produites dans le nord, et, de préférence, dans la localité.

Variétés: Peu de cultivateurs canadiens établissent de différence entre les variétés d'herbes et de trèfles communs. En fait, on connait peu les variétés de mil, de dactyle pelotonné, de ray-grass de l'Ouest, de trèfle rouge hâtif, de trèfle d'alsike et de luzerne; du reste, hors quelques espèces de luzerne, ces variétés ne se trouvent pas dans le commerce. Elles existent depuis peu, mais elles présentent de remarquables différences au point de vue de la précocité, du rendementet de la qualité générale. Dès que l'on pourra se procurer de la bonne 
graine des meilleures variétés, les cultivateurs trouveront avantageux de s'en servir au lieu d'employer de la graine ordinaire de commerce.

Le pourcentage de vitalité dans la graine de graminées et de trèfles est un facteur important et qui exige une attention toute spéciale surtout dans les graminées d'espèce plus fine. Au moins quatre-vingtquinze pour cent des graines de plantes fourragères employées au $\mathrm{Ca}$ nada se composent de mil, de dactyle pelotonné, brome inerme, raygrass de l'Ouest,trèfle rouge, trèfle d'alsike et luzerne, et à l'exception de celle du brome inerme la graine de ces espèces que l'on trouve dans le commerce est généralement douée d'une forte vitalité. La bonne semence de brome inerme, de pâturin, de fétuque et des autres herbes plus fines devrait germer dans la proportion de quatre-vingt pour cent au plus mais les échantillons de commerce contiennent souvent moins de cinquante pour cent de semence viable. La graine qui germe dans la proportion de quatre-vingt pour cent revient moins cher en réalité à trente cents la livre que la graine qui se vend la moitié de ce prix et dont le pourcentage de germination est proportionnellement plus faible. Les bons grainetiers connaissent la vitalité de leurs graines mais les acheteurs de plantes plus fines devraient faire leurs achats au moins un mois avant l'époque des semis et essayer leurs graines. Voici la façon de procéder: on sème deux cent graines moyennes de chaque espèce dans un pot de fleurs, rempli d'une terre légère, et on les tient légèrement humides à la température d'une chambre d'habitation, près d'une fenêtre ensoleillée, pendant environ trois semaines.

Pureté: La valeur des graines de graminées et de trèfles se ressent principalement de la nature et de la quantité des impuretés qu'elles renferment. Malheureusement il est difficile d'obtenir des graines qui ne contiennent aucune semence de mauvaise herbe. Or cent graines de mauvaises herbes peuvent fort bien passer inaperçues dans une once de graine d'herbe ou de trèfle mais il en est tout autrement dans la récolte qui lève. Il est rare que l'on apprécie entièrement tous les mauvais effets que ces herbes peuvent exercer sur le bétail. Dans tous les cas, c'est faire preuve d'un grand manque de jugement que d'acheter de la graine de qualité inférieure. La meilleure graine revient toujours le moins cher à la longue.

Le moyen le plus efficace et le plus économique de détruire les mauvaises herbes dans la prairie est de bien préparer la terre avant d'enherber. Les herbes vivaces comme la grande marguerite, le chardon, la silène et le chiendent tendent à se propager dans les prairies. Dans un climat humide on peut, en laissant la terre en gazon 
pendant cinq ans ou plus, empêcher certaines herbes annuelles ou bisannuelles comme la folle avoine et la vipérine de monter à graine. Dans un climat sec, les graines de mauvaises herbes enfouies dans le sol conservent leur vitalité plus longtemps. On peut réduire le nombre des moutardes, des herbes à poux et autres annuelles en laissant la terre en prairie ou pâturage pendant un certain nombre d'années, mais il est bien difficile d'empêcher des plantes isolées de produire quelques graines tous les ans.

Dans une terre assez propre que l'on veut mettre en prairie, l'emploi d'une céréale comme plante-abri retarde généralement la végétation des mauvaises herbes et les empêche de mûrir avant la récolte. Il faut pour cela que la plante-abri soit prête à être récoltée ou à être coupée comme fourrage dans un délai de trois mois ou au plus de trois mois et demi après les semis. Si les mauvaises herbes ne sont pas très nombreuses lorsqu'on récolte la plante-abri, il vaut mieux, pour protéger les jeunes herbes, laisser un chaume de quatre à cinq pouces de hauteur. On enlève ainsi les tiges à graines des mauvaises herbes les plus élevées et les plus vigoureuses, et on permet aux jeunes plantes fourragères, encore délicates, de s'adapter graduellement au changement de conditions. On peut empêcher les mauvaises herbes d'automne de répandre leurs graines en coupant avec une faucheuse un mois environ après que la plante-abri a été récoltée. Cette précaution est spécialement à recommander dans le cas d'une terre infestée d'herbe à poux.

Dans le développement d'une prairie il arrive fréquemment que la jeune herbe souffre beaucoup de la mauvaise température, de l'irrégularité des semis, de l'humidité ou de la sécheresse trop grande de certaines parties du champ, ou encore de la verse partielle d'une plante-abri trop serrée. Souvent de petites superficies sont détruites. Dès que les pluies de l'automne commencent, ou, si le sol est suffisamment humide, dès que les chaleurs de l'été sont passées, il est bon de resemer ces parcelles en mettant beaucoup de graine. On applique, s'il est nécessaire, une mince couche de fumier pourri pour recouvrir les graines, retenir l'humidité et provoquer une vigoureuse germination d'automne. Si les superficies détruites sont considérables, il est parfois bon, avant de semer, de passer une herse pointue pour préparer la terre à recevoir la graine. Si la fin de l'automne est favorable et que les parcelles resemées soient bien protégées pendant l'hiver, la pousse sera bonne, même pour la première' coupe, et les récoltes suivantes dédommageront amplement du travail et des frais. 
Mais il ne suffit pas d'extirper les mauvaises herbes, il faut aussi stimuler la pousse des bonnes; un bon moyen de le faire est de couper la jeune herbe à la faucheuse vers la troisième semaine de septembre au plus tard, soit environ un mois après que la plante-abri a été récoltée. Cette opération encourage les trèfles et les graminées à taller et à ramifier, et la récolte est plus épaisse et la végétation plus uniforme le printemps suivant. En enlevant le chaume de la plante-abri et les mauvaises herbes qui ont poussé en automne, on obtient, à la première coupe, du foin plus propre et de meilleure qualité. Mais il importe que l'on passe la faucheuse aussi tôt que possible afin que les plantes aient le temps de faire une pousse suffisante pour protéger leurs racines pendant l'hiver. Pour la luzerne, la dernière coupe ne devrait pas être faite plus tard que la troisième semaine d'août. On ne devrait jamais faire pâturer les jeunes prairies après cette coupe d'automne. Au début du printemps suivant, si la terre est suffisamment égouttée, il est souvent avantageux de passer un lourd rouleau.

Sur les sols plus secs des prairies, où l'on ne peut se servir de plante-abri, deux ou trois fauchages feront disparaître les mauvaises herbes et conserveront l'humidité, mais il ne faut pas faucher après le milieu d'août.

Le manque de protection en hiver pour les jeunes prairies est la cause la plus commune des pauvres rendements et de la mauvaise qualité du foin. Pendant la saison sèche, lorsque les pâturages naturels et les récoltes fourragères sont maigres, il semble parfois que l'on ne puisse se dispenser de faire usage, dès l'enlèvement de la plante-abri, des prairies nouvellement semées, même lorsque les jeunes plantes luttent encore pour l'existence et sortent affaiblies de leur lutte avec la plante-abri qui les a dépouillées d'humidité plutôt qu'elle ne les a protégées. C'est justement dans des conditions de ce genre que la paissance est désastreuse. Pour chaque livre de fourrage tirée des jeunes plantes, on perd plus de dix livres dans la récolte de foin; la récolte est moins épaisse et la qualité du foin plus pauvre. Les jeunes plantes devraient complètement recouvrir le sol et avoir une hauteur de six pouces ou plus avant la fin de l'automne. Le seul avantage qu'il puisse y avoir à faire paître une jeune prairie la première année est lorsqu'il y a danger qu'une épaisse végétation de trèfle n'étouffe la récolte, mais ce cas se voit rarement.

Il faut couper les graminées et autres plantes fourragères lorsque la récolte a atteint son maximum de valeur en rendement et en qualité. L'effet de l'époque de la coupe sur le regain ou sur les récoltes 
suivantes est également à considérer. La fonction principale d'une plante est de se reproduire. Jusqu'à ce que ses organes porteurs de semence aient été fécondés, elle ramasse de la nourriture et l'emmagasine dans ses tissus pour former et mûrir ses graines. Dès que la fleur est fécondée, les graines tirent sur la provision de nourriture que contiennent les tiges et les feuilles et la plante commence à durcir. Chez quelques espèces de plantes fourragères, telles que le pâturin, qui comptent principalement sur leurs racines pour se reproduire et qui ne portent que quelques graines, ce durcissement de la piante n'est pas aussi prononcé, mais dans presque toutes les espèces les plus précieuses, le changement de tissus succulents et souples en tissus cassants et ligneux est rapide et marqué. Même avant la fécondation, beaucoup de plantes fourragères comme la iuzerne, le ray-grass de l'Ouest et le mil commencent à durcir.

Si l'on coupe avant que les fleurs soient prêtes à être fécondées, ies plantes font de nouveaux efforts pour se reproduire et le regain n'en est que plus épais. Si l'on retarde la coupe jusqu'à ce que les graines aient commencé à se développer, la tendance naturelle des plantes fourragères bisannuelles et du trèfle rouge en particulier est de mourir; dans le mil et les autres graminées l'effet est apparent, non seulement dans leur regain, mais aussi dans la récolte de l'année suivante. Dans les champs laissés à eux-mêmes la récolte de l'année suivante ne se composerait en partie que de jeunes plantes provenant de graines qui, dans la culture, se forment assez souvent mais n'ont pas le temps de mûrir.

Au point de vue de la qualité du foin il y a beaucoup à perdre et rien à gagner à retarder la coupe jusqu'à ce que la floraison soit bien avancée. Le rendement par acre augmente un peu dans les quelques jours qui se passent entre les premières et les dernières fleurs, mais cette petite augmentation est obtenue aux dépens d'une dépréciation sensible de qualité; d'autre part, si l'on considère le regain et les récoltes suivantes, le rendement total est en réalité réduit.

Dans un pré formé d'un mélange de plantes fourragères qui fleurissent à époques différentes, comme par exemple le trèfle rouge hâtif et le mil, le moment le plus propice pour faire la première coupe dépend de la proportion de chaque plante dans la récolte totale. On trouvera généralement avantageux, et, à la longue, plus économique, de couper dans les deux ou trois jours qui suivent le moment où le trèfle a atteint cette phase de son développement qui permet d'en'tirer le meilleur foin. Par une température chaude et sèche, l'herbe mûrit rapidement et un délai de quelques jours peut causer 
tout autant de mal qu'une période beaucoup plus longue par un temps frais et sur un sol humide.

Pour le foin il vaut mieux couper à la faucheuse; pour une récolte de graine on emploie généralement la moissonneuse; on assemble les gerbes en petites moyettes pour que la graine sèche et finisse de mûrir.

On recommande de couper le foin au ras du sol. Lorsque la récolte se compose principalement de trèfle, qu'elle est très forte et versée par endroits, il faut ajuster la faux de façon à couper au bas des tiges; sinon le chaume restant constituerait un danger pour les faneuses mécaniques et les râteaux qui vont suivre et ne laisserait qu'un fourrage sans valeur à récolter avec le regain. C'est lorsqu'il faut couper une forte récolte de trèfle très versée que l'on apprécie l'avantage d'avoir une surface bien unie, produite par l'emploi d'une herse souple après les semis et d'un rouleau en travers des raies au printemps.

On fauche généralement pendant la partie du jour où la rosée empêche de faner et de charroyer. Mais lorsque la récolte de foin est très forte, qu'elle est humide de rosée et qu'elle colle sur la faux, il vaut mieux couper vers la fin de l'après-midi. Le fanage, c'est-àdire le retournement de l'herbe verte, devrait être commencé immédiatement après la coupe. Si la récolte est forte, on doit continuer à faner par intervalles jusqu'à ce que le fourrage soit assez sec pour pouvoir être râtelé en rangs et mis en petites veillottes. On devrait, autant que possible, faire ces opérations le jour même où le foin est coupé ou le jour suivant, s'il est coupé dans l'après-midi. Le fourrage vert, coupé au moment le plus propice pour la fenaison, contient généralement environ quatre-vingt p.c. d'eau. Si le temps est beau il suffit d'un jour pour sécher une récolte de trèfle suffisamment pour qu'elle puisse être mise en veillottes, dans lesquelles l'herbe achève de se faner.

La proportion d'eau dans le foin prêt à engranger varie généralement de douze à quinze pour cent. Un foin plus humide serait exposé à ressuer et à chauffer dans les tasseries. Une bonne méthode est de couper jusqu'à neuf heures du matin, puis de mettre un homme à faner et à râteler pour le reste du jour. Les charroyages et l'engrangement se font à partir de neuf heures du matin jusqu'à quatre heures ou quatre heures et demie de l'après-midi. On emploie les deux heures qui restent à mettre en veillottes le foin qui vient d'être fané. Parfois, cependant, les dispositions prises sont déran- 
gées par les orages qui augmentent le travail et sont souvent plus désastreux à la qualité du foin que les chaleurs excessives.

Même pendant les longues pluies il est bon de secouer le foin à moitié fané en le retournant à la fourche ou à la faneuse afin de l'empêcher de se tasser et de se remplir d'eau. Le foin est plus exposé à perdre son goût et sa valeur nutritive s'il repose en une masse humide et compacte que s'il est tenu lâche, quand bien même il est mouillé. Par une journée sèche et chaude, il importe de couper et de faner promptement. Le foin séché au grand soleil est exposé à perdre beaucoup de sa qualité, il est meilleur lorsqu'il est fané sous un vent léger. Par un temps chaud et sec, il est bon d'employer la faneuse immédiatement après la coupe et à intervalles fréquents, et de râteler et de mettre en veillottes lorsque le fourrage est encore très humide. Lorsque la maturation se fait rapidement, il vaut souvent mieux remettre les charroyages pour faucher et faner. On met alors le foin en grosses veillottes.

Les méthodes de fanage et de charroyage se sont modifiées à mesure que la main d'œuvre se faisait plus rare et plus chère. On ne met plus le foin en veillottes depuis que l'on emploie la chargeuse. On charge du rouleau même, dès que le foin est assez fané; cette méthode donne de bons résultats.

La fenaison n'est plus un travail pénible de nos jours, bien différent de celui auquel étaient astreints les premiers colons qui coupaient l'herbe à la faucille, retournaient le foin à la fourche, le râtelaient avec des râteaux en bois et le chargeaient et le déchargeaient à la main. Dix acres en foin représentaient une entreprise assez considérable pour le pionnier; aujourd'hui son petit-fils avec moins d'aide mais plus de machines, récolte facilement cinq fois cette superficie. Les souches et les pierres ne gênent plus le passage de ses machines; son grand-père en a fait des clôtures. Assis sur sa faneuse mécanique il peut retourner autant de foin en une heure que son arrière grand'mère aidée de ses filles pouvait faire en un jour. Aujourd'hui le râtelage, le chargement et le déchargement se font presque entièrement avec le cheval.

L'effet des graines de mauvaises herbes: Avec l'évolution des machines et des moyens de transport sont venues les mauvaises herbes qui se sont répandues partout. Peu de gens se rendent compte des pertes que causent les mauvaises herbes dans nos cultures fourragères: On apprécie facilement la dépréciation qui résulte de leur présence dans un champ de grain, il n'en est pas de même dans le 
foin dont le rendement total peut être réellement augmenté par leur présence.

C'est dans les pâturages infestés que l'on peut le mieux étudier les mauvaises herbes. On remarquera que plusieurs espèces, évitées par le bétail paraissent moins répugnantes aux chevaux et sont même parfois recherchées par les moutons. Il en est de très vénéneuses, comme le panais d'eau, d'autres, comme les moutardes, les marguerites et les bardanes, ne deviennent dangereuses que lorsqu'elles sont consommées en quantités considérables ou pendant une longue période. On s'aperçoit de leur nature vénéneuse par la mauvaise santé persistante des animaux. Dans un pâturage, il est rare que les bestiaux consomment assez de mauvaises herbes pour devenir malades, à moins que les bonnes ne fassent complètement défaut. Quand on les laisse fourrager dans le champ, les animaux, et particulièrement les bêtes à cornes, se portent généralement mieux que lorsqu'on les rationne à l'étable, même avec une nourriture plus nutritive.

Personne n'ignore la saveur âcre du sysimbre officinal, de la cameline, de la bourse à pasteur et d'autres membres de la famille des moutardes, ces plantes contiennent un violent irritant; ceux qui connaissent la sensation causée par un cataplasme de moutarde n'auront pas de peine à comprendre les effets que ces plantes peuvent produire lorsqu'elles sont consommées en quantité avec des fourrages hachés. Données pendant longtemps et avec du foin ou du grain, leurs effets sont moins prononcés. On les remarque tout d'abord dans l'urine; puis des ulcères profonds se forment sur l'animal, semblables parfois à ceux produits par des applications prolongées de cataplasmes de moutarde et qui se guérissent difficilement.

La plupart des membres de la famille des lychnides contiennent de la saponine qui est nettement vénéneuse. Les animaux qui mangent du foin infesté de lychnide ne meurent pas, car la proportion de saponine n'est pas suffisante pour les tuer, mais ils sont en mauvais état de santé, la digestion est imparfaite, l'appétit mauvais, ils sont faibles, la peau est brâlante et ils maigrissent graduellement.

Les boutons d'or sont très âcres et causent des plaies dans la bouche des animaux; les bestiaux ne veulent pas paître dans les prairies qui en sont infestées. Consommés en grande quantité ou pendant longtemps, les boutons d'or causeraient, dit-on, l'avortement chez les vaches.

Plusieurs membres de la famille des tournesols ne sont pas sains et quelques-uns d'entre eux sont tout à fait vénéneux. L'herbe à 
poux est un irritant; on croit que son pollen cause la fièvre des foins. L'herbe de St.-Jacques (Senecio Jacobra), commune dans certaines parties des provinces de l'Atlantique, est également la cause de la maladie du bétail de Pictou. Cette herbe, semblable en cela à beaucoup d'autres plantes, qui sont vénéneuses pour certains bestiaux et sans danger pour d'autres, ne fait aucun mal aux moutons.

Les animaux à l'étable auxquels on donne du foin rempli de mauvaises herbes et qui n'ont pas d'autre alternative que de le manger ou mourir de faim, le fouillent généralement et ne mangent que la partie savoureuse. Pour prévenir cette perte on hache leurs fourrages. Ce foin haché est souvent malsain à cause des mauvaises herbes qu'il renferme. Les vaches laitières ne le mangent que poussées par la faim et ne donnent souvent qu'un gallon de lait d'odeur désagréable au lieu de trois gallons de bon lait qu'elles pourraient donner par jour. Sur une ferme infestée de mauvaises herbes les bestiaux, et particulièrement les bêtes à cornes, sont dans un état chronique de mauvaise santé et de maigreur. Les mauvaises herbes diminuent donc beaucoup la valeur d'une récolte fourragère quand elles ne la détruisent pas entièrement. En établissant une prairie, il est donc très important de faire disparaître les mauvaises herbes dangereuses avant de semer la graine d'herbe.

La durée des prairies et des pâturages dépend du mode d'exploitation adopté, de la nature du sol et du drainage. Pour les terres élevées à bon égouttement naturel et soumises à une culture mixte, on recommande de courtes rotations qui comportent deux ans en gazon de trèfle rouge et de graminées. Immédiatement après avoir enlevé la récolte de foin de la deuxième année, on peut labourer la prairie et la mettre en jachère pour le reste de l'année afin d'extirper les mauvaises herbes. Une application de fumier de ferme, incorporée au sol par un labour mince ou un hersage, devrait mettre le terrain en état de porter une culture sarclée l'année suivante; cette culture sera suivie, l'année d'après, par une céréale dans laquelle on sèmera de nouveau du trèfle rouge et des graminées qui resteront deux ans sur le sol.

La rareté de la main-d'œuvre a fait adopter une culture moins intensive dans certains districts. La luzerne donne de plus forts rendements et exige beaucoup moins de travail. Il existe des espèces rustiques, notamment de la luzerne rustique ou moyenne, et les cultivateurs qui se procurent des semences appartenant aux meilleures espèces, produites dans le nord, peuvent compter obtenir des récoltes satisfaisantes plusieurs années de suite, pourvu que la terre soit bien égouttée et qu'elle ne soit pas infestée de mauvaises herbes vivaces. 
Dans les districts où la neige recouvre la terre et protège la récolte, l'hiver est moins à craindre. Dans la péninsule de Niagara on voit des luzernières de luzerne rustique de plus de trente ans qui produisent encore beaucoup de fourrage. La luzerne qui n'est pas bien protégée est exposée à être détruite par les hivers rigoureux, et peu de champs continuent à donner de bonnes récoltes pendant plus de cinq ou six ans.

Dans les sols argileux et humides, les bords de rivières, il est souvent nécessaire ou il peut être plus commode de laisser la terre en prairie ou en pâturage permanent pendant de longues périodes. Il est difficile, au commencement du printemps, de préparer des fonds humides à la culture des céréales et les bords de rivières qui ne sont pas enherbés sont exposés à être inondés et creusés par l'eau. Le sédiment déposé par les inondations de printemps entretient généralement la fertilité de ces terres, et si l'on réussit à y établir les herbes les plus convenables, on peut en attendre de forts rendements de bon foin pendant bien des aṇnées.

L'engraissement des prairies de longue durée se pratique généralement en Europe. Il se fait un peu dans les provinces de l'Est du Canada mais très peu à l'intérieur du pays. Une application de fumier de ferme, bien pourri, épandu tous les deux ou trois ans, au commencement du printemps, fait beaucoup de bien. C'est le meilleur moyen de tenir une prairie en bon état. Le fumier en décomposition, épandu à la surface, forme un paillis qui retient l'humidité. Les trèfles sont souvent avantagés par l'apport de potasse, de chaux, sous forme de plâtre, etc., mais ils répondent peu aux fumures azotées. Les vieilles prairies répondent rapidement quand on leur applique, au commencement de la saison, du nitrate de soude à raison de cent livres par acre. Sur les terres basses, naturellement humides, on peut obtenir de bons rendements en semant, tous les deux ou trois ans, trois cents ou quatre cents livres, par acre, d'un engrais mélangé ou de poudre d'os riche en azote.

Comparés aux sols bien cultivés et soumis à un assolement régulier, les pâturages permanents ne donnent qu'un bien faible revenu. Dix acres de bonne luzernière coupés en vert produiraient autant de fourrage nutritif que quarante acres de pâturage permanent. Les pertes résultant du piétinement des animaux sont beaucoup plus fortes dans les prairies temporaires de trèfle et de mil que dans les prairies permanentes, composées d'un mélange d'herbes, mais le rendement des premières est généralement beaucoup plus élevé et le fourrage plus accessible au bétail. Les pâturages permanents sont surtout utiles pour les moutons. On ne les recommande pas pour 2855)-3 
les bêtes à cornes sur les terres faciles à cultiver, à moins qu'il ne soit impossible de se procurer la main-d'œuvre nécessaire.

Il est rarement nécessaire de réensemencer ou de renouveler une prairie qui est bien entretenue et bien protégée en hiver. Toutefois on constate, sur certains sols, que lorsque l'on sème plusieurs sortes de graminées et de trèfles une ou deux espèces dominent à l'exclusion des autres. Si l'on a besoin d'une prairie ou d'un pâturage permanent de longue durée, il peut être nécessaire de semer d'autres graminées pour compléter celles qui ont déjà pris racine. Il faut les choisir avec soin et dans un but bien arrêté; par exemple, l'agrostide pourra être choisie pour les fonds ou les terres humides, où toutes les autres espèces, à l'exception du mil, ont été détruites. On sème au début du printemps et si la terre est assez sèche on peut passer une herse pointue suivie d'un lourd rouleau pour recouvrir la graine et obtenir une surface lisse.

Les pentes de collines et les endroits exposés dans les prairies nouvellement formées, de même que dans les prairies et les pâturages de longue durée, ont souvent besoin d'être renouvelés et réensemencés après un hiver rigoureux. L'épandage d'une bonne quantité de semence, suivi de la herse ou du rouleau, ou des deux, donne généralement de bons résultats. Si la terre est sujette à durcir ou à se prendre en croûtes sur les superficies réensemencées, on fera bien de mettre une légère application de fumier d'étable bien pourri en couverture.

L'emploi du rouleau au printemps est bon, aussi bien pour les prairies nouvelles que pour les vieilles prairies, surtout celles qui ont gelé et dégelé à plusieurs reprises au commencement du printemps.

\section{LE BLÉ D'INDE (Zea Mays L).}

Autres noms français: Maïs, blé de Turquie, blé d'Espagne, gros millet.

Noms anglais: Corn, Indian Corn, Maize.

Description botanique: Le blé d'Inde ou maiss est une des graminées annuelles les plus élevées et les plus vigoureuses. Les tiges, dont la hauteur varie dans les divers types et variétés, sont pleines, tandis que celles de la plupart des autres graminées sont creuses. Les feuilles, longues, larges, ondulées, se terminent gra- 
duellement en pointe à l'extrémité. Au sommet de la tige se trouve une forte panicule, à rameaux étalés et chaque rameau porte de nombreuses fleurs. Ces fleurs ne contiennent que les étamines ou organes mâles et ne peuvent normalement former de graines. Les fleurs femelles, qui ne contiennent que des pistils, ou organes femelles, sont disposées en rangs sur des épis charnus, à l'aisselle des feuilles moyennes. C'est sur ces épis que se forment les graines. Au début les épis naissants sont enveloppés d'une gaine ou spathe de larges feuilles et les fleurs sont invisibles. Au moment de la floraison, une houppe de fils soyeux, longs et minces, d'un vert jaunâtre ou rougeâtre, sort du bout de l'épi. Ces fils, appelés les 'soies' sont les extrémités des fleurs femelles; ils attrapent le pollen poussiéreux qui se forme dans les fleurs mâles et que le vent dissémine. De même que dans les autres inflorescences de graminées, l'épi commence à se développer à la base et croît en montant. Aussi les premières soies visibles appartiennent aux fleurs les plus basses; dans des conditions normales, celles-ci sont donc fécondées avant les fleurs supérieures. Si, pendant la dernière partie de la floraison, la température est défavorable, le pollen n'est pas transporté et déposé sur les soies, et la partie supérieure de l'épi reste partiellement ou entièrement stérile car les graines ne peuvent se former si les fleurs ne sont fécondées.

Historique et distribution géographique: Le blé d'Inde est évidemment d'origine américaine. Les Indiens le cultivaient bien avant la découverte de l'Amérique. On dit que les Incas du Pérou construisaient de grands entrepôts pour en faire des provisions en cas de famine. Les premiers explorateurs du Canada l'ont rencontré jusque dans la vallée du Saint-Laurent. On trouve souvent des épis de blé d'Inde dans les vieux sépulcres indiens. Ils ont été déposés là avec les morts, comme provision pour le long voyage aux heureux terrains de chasse.

On ne sait au juste où et quand il a été cultivé en premier lieu ni de quelle plante sauvage il descend. On croit généralement que sa culture a pris naissance dans le centre de l'Amérique et s'est propagée au nord et au sud. On n'en a jamais trouvé à l'état sauvage. On peut en conclure que le maïs sauvage avait disparu avant que les botanistes en aient fait la recherche, ou que c'est une plante si différente de l'espèce cultivée qu'il est impossible de l'en distinguer aujourd'hui. Cette dernière supposition est la plus généralement adoptée et la plante mentionnée comme l'espèce primitive probable est le Téosinte mexicain (Euchlaena mexicana Schrad.). Quoique 
très différent du blé d'Inde au point de vue de l'apparence générale le Téosinte s'en rapproche cependant beaucoup. Les hybrides obtenus par le croisement des deux espèces produisent des graines germables. Ce n'est pas là, à vrai dire, une preuve concluante que le blé d'Inde descend du Téosinte, mais c'est une indication, car dans tous les autres cas connus les hybrides des espèces distinctes de graminées sont stériles.

Climat: Le blé d'Inde vient du sud; il exige donc un climat chaud et humide. Dans le nord, où les saisons sont courtes et la température relativement fraîche, il n'y a que les variétés les plus hâtives qui puissent arriver à maturité dans des conditions ordinaires.

Sol: Il exige un sol fertile et chaud et donne ses meilleures récoltes sur une terre franche, grasse, riche, profonde, bien égouttée, mais contenant une quantité abondante d'eau. Une bonne provision de matière organique, qui fournit des principes nutritifs facilement assimilables, augmente beaucoup le rendement. Les sols sablonneux, pauvres, ou ceux dont le niveau d'eau est très près de la surface, ne permettent pas aux racines de recueillir assez de nourriture. Dans les argiles lourdes ou dans les terrains qui reposent sur un sous-sol imperméable, la végétation est lente et le rendement peu sûr, principalement pendant les sécheresses.

Variétés: Il y a des centaines de variétés agricoles de blé d'Inde. Cette abondance de variétés s'explique par la promptitude avec laquelle les croisements se produisent entre les sujets des divers types. Certaines espèces sont naines n'ayant pas plus de dix-huit pouces de hauteur, d'autres sont géantes, atteignant une hauteur de vingt à vingt-cinq pieds. Dans quelques-unes, les épis n'ont qu'un pouce ou deux de longueur; dans d'autres ils ont jusqu'à seize pouces. Le nombre de rangs de grains, qui est toujours pair, varie de huit à vingt-quatre au plus, suivant la variété. Dans certaines espèces on rencontre des épis anormaux qui n'ont que quatre rangs, tandis que d'autres, plus développés, en ont jusqu'à quarante-huit. La grosseur des grains, leur forme, couleur, composition chimique, etc., sont extrêmement variables. D'après le Dr. E. L. Sturtevant, les variétés peuvent être classées en sept groupes principaux que voici :

r. Les blés d'Inde à gousse (pod corn) où chaque grain est tenu dans une gousse ou petite spathe et où l'épi est également enveloppé 
de spathes. Dans tous les autres groupes les grains sont nus dans les spathes. On doute cependant que les blés d'Inde à gousses forment un groupe naturel. Peut-être les spathes qui entourent les épis sont anormales et pourraient se rencontrer dans tous les groupes principaux. Cette opinion s'appuie sur le fait que la structure du grain varie dans les blés d'Inde à gousse.

2. Les blés d'Inde soufflés ( $p o p$ corn) se caractérisent par une proportion excessive de l'endosperme corné, c'est-à-dire la matière nutritive qui forme la plus grande partie du grain et qui est emmagasinée pour servir aux germes; elle contient peu d'amidon. Dans ce groupe les grains et les épis sont petits. La faculté qu'ils possèdent d'éclater quand on les met sur le feu, c'est-à-dire de se retourner complètement, le dedans en dehors, sous l'explosion de l'eau qu'ils renferment, est plus prononcée dans les variétés qui ont un endosperme corné sur toute leur surface et il est moins prononcé dans celles qui contiennent un pourcentage plus élevé d'amidon.

3. Les blés d'Inde vitreux (fint corn) chez lesquels la partie centrale de l'endosperme est féculeuse et complètement entourée d'une enveloppe cornée dont l'épaisseur varie suivant les variétés. Jacques Cartier a trouvé des variétés de ce groupe dans le voisinage de Montréal.

4. Les blés d'Inde cochés (dent corn) chez lesquels la partie féculeuse centrale de l'endosperme est entourée d'une couche cornée sur les côtés du grain seulement. L'endosperme féculeux se prolonge ainsi jusqu'au sommet de l'épi. Lorsque cet endosperme sèche et se retire, il se produit des creux ou "coches" sur le sommet du grain. Les blés d'Inde cochés sont très cultivés aux Etats-Unis. Le nombre de variétés de ce groupe dépasse celui de toutes les autres variétés ensemble.

5. Les blés d'Inde mous (soft corn) qui n'ont pas d'endosperme corné et chez lesquels le retrait, au cours de la maturation, est uniforme dans toutes les parties du grain. A ce groupe appartiennent les blés d'Inde momies du Pérou et du Chili.

6. Les blés d'Inde sucrés, (sweet corn) qui sont caractérisés par des épis translucides, cornés, et une surface plus ou moins plissée, ridée ou fanée. Ils sont très cultivés pour les conserves, surtout dans les parties est du nord de l'Amérique. 
7. Les blés d'Inde sucrés féculeux (starchy-sweet corn) chez lesquels la partie inférieure du grain est féculeuse tandis que la partie supérieure est cornée et translucide. Ce groupe est peu connu.

Valeur agricole: Lorsque Christophe Colomb débarqua aux Antilles on lui présenta une sorte de pain fait avec un grain que les indigènes appelaient 'mahiz'. C'est de ce mot que viennent les noms français 'maïs', et anglais 'maize', sous lesquels la plante est connue en Europe. Colomb remporta du maïs avec lui en son pays mais on fût lent à apprécier la plante en dehors de l'Espagne et du Portugal. On la cultive maintenant dans toute l'Europe, surtout en Italie, où le gruau de blé d'Inde (polenta) est la nourriture habituelle du travailleur; en Espagne où les gâteaux de farine de blé d'Inde (tortellas) sont très importants, et dans les pays situés le long du bas Danube. Plus tard sa culture s'est répandue dans toute l'Europe, l'Inde et toute l'Afrique. Toutefois elle n'a pas dans ces pays la même importance qu'en Amérique. Dans l'Amérique du Sud et du centre et dans les Etats-Unis le blé d'Inde est cultivé pour la production du grain et du fourrage. Son importance comme plante fourragère croît à mesure que l'on avance vers le nord. Vers les limites de la zone à blé d'Inde, il est cultivé principalement pou: la production du fourrage.

Fourrage: On donne généralement le blé d'Inde vert pour suppléer au manque de pâturage vers la fin de l'été ou en automne. Tous les bestiaux en sont friands, mais particulièrement les bovins. Parfois on le coupe en vert et on le fait faner mais il retient beaucoup d'humidité et se conserve difficilement pour l'alimentation d'hiver.

Quand on le cultive pour la production du grain, le fourrage sec dont on a enlevé le grain mûr est dur et ligneux. On peut le hacher, l'humecter et le laisser en tas jusqu'à ce qu'il commence à fermenter, alors il devient plus succulent et forme une nourriture économique et saine pour les bêtes de rente. Toutefois il n'a pas la valeur nutritive du blé d'Inde coupé deux semaines plus tôt et que l'on ensile avec le grain.

Au Canada le blé d'Inde est cultivé comme plante d'ensilage presqu'à l'exclusion de toutes les autres plantes. Même aux limites nord de la zône à blé d'Inde, les variétés hâtives 'Flint' naines telles que le jaune à huit rangs, donnent plus de nourriture par acre que toute autte plante fourragère. Dans une localité quelconque, le type et la variété les plus propres à la production de l'ensilage varient suivant 
la durée de la saison de végétation et la chaleur naturelle du sol. C'est le blé d'Inde qui arrive à l'état lustré, c'est-à-dire à cette phase de la maturation ou le grain commence à se recouvrir d'une croâte dure à la surface, qui fournit le plus de nourriture à la tonne. C'est ce blé d'Inde qui renferme le plus de protéine, l'élément formateur de chair et la protéine de la meilleure qualité. Au début de la maturation, les éléments azotés dont cette protéine est formée se trouvent sous forme liquide et n'ont qu'une faible valeur nutritive. L'ensilage fait avec du blé d'Inde qui n'a pas dépassé le début de l'état laiteux est généralement sur, et, malgré sa succulence, c'est une pauvre nourriture pour le bétail, bien inférieure au blé d'Inde qui a presque atteint la maturité.

Il est donc très important de choisir une variété de blé d'Inde qui arrive à l'état lustré même dans des conditions légèrement défavorables de température et assez tôt pour qu'il n'y ait rien à craindre des gelées. C'est là la première considération dans le choix d'une espèce. La quantité de tiges et de grain produite vient ensuite par ordre d'importance. Règle générale, la variété la plus avantageuse que l'on puisse cultiver pour l'ensilage sur un sol ordinaire, c'est-à-dire la variété qui donne le plus de nourriture à l'acre, est celle qui mûrit parfaitement sur un sol plus chaud de la même localité où sur un sol semblable à quarante ou cinquante milles plus au sud.

L'expérience démontre invariablement qu'il est sage, pour la production de l'ensilage, de choisir des variétés hâtives plutôt que des variétés tardives à gros rendement.

Pour la production du fourrage on plante généralement le blé d'Inde en rangs, à raison de douze à vingt pintes de bonne semence à l'acre. L'écartement entre les rangs ne doit jamais être de moins de trente-six pouces pour les variétés hâtives et courtes, et de quarantedeux pouces pour les variétés tardives et élevées.

Quand on plante deux ou plusieurs variétés de blé d'Inde pour l'ensilage, il est bon de les planter séparément, surtout si l'une des espèces est plus élevée ou fleurit plus tard que l'autre. Quand les variétés plus petites et plus hâtives sont plantées en mélange avec les espèces plus développées et plus tardives, les premières sont généralement mal fécondées et leur rendement de grain en souffre.

Semence: Il faut s'attacher à prévenir les croisements entre les variétés. Le vent transporte le pollen à de longues distances et les semences de variétés cultivées dans un rayon de 400 verges l'une de l'autre sont sujettes à être plus ou moins impures. 
Le blé d'Inde égrené, de même que le blé d'Inde en épis, retient beaucoup d'humidité; à moins que l'on ait pris des soins tout spéciaux pour faire sécher la semence avant de l'emmagasiner, sa vitalité peut souffrir beaucoup d'une forte chaleur ou d'une forte gelée. Le blé d'Inde tout à fait mûr et destiné à la production de la semence devrait être coupé et séché sur la tige avant d'être épluché, c'est-à-dire dépouillé de ses spathes. Si le temps est humide, peu favorable au fanage, il faut employer des moyens artificiels pour faire sécher la semence sur l'épi. On doit la protéger contre la gelée jusqu'à ce que la rafle (coton) soit tout à fait séchée et cassante. Un germe noir, à enveloppe ridée, indique que la graine a souffert de la gelée.

\section{MILLETS.}

Les millets comptent parmi les plantes les plus anciennes en agriculture. On les cultive depuis un temps immémorial en Asie et dans certaines parties de l'Europe, où la graine sert principalement à l'alimentation de l'homme. En Amérique ils servent à la production du fourrage. Au Canada ils sont rarement employés dans les assolements réguliers mais on les utilise comme cultures dérobées.

Tous les millets exigent une terre franche, riche, contenant beaucoup de principes fertilisants, près de la surface, et une bonne quantité d'eau. Dans des conditions favorables la végétation est rapide et l'on obtient une récolte serrée en peu de temps. La récolte peut être utilisée sous forme de foin, de pâturage ou d'ensilage. Les nombreuses variétés cultivées au Canada et aux Etats-Unis appartiennent à quatre espèces très différentes d'aspect général.

Quelle est la première chose? Bien cultiver. Quelle est la seconde? Bien labourer. Quelle est la troisième? Fumer.-Un champ, s'il n'est pas fumé se refroidit; sl on le fume trop, ll est brale; il vaut mieux fumer souvent qu'avec excès.-Pline, Histoire naturelle, 23-79 AC.

Apprenez qu'il n'y a qu'une chose en culture.-Avec elle tout vient et sans elle on n'a rien...... . Qu'est-ce que c'est, demandent-ils?. . Du fumler, répond Liset. . C'est le maltre laboureur, c'est le grand cultivateur. Dans la plaine, on croit s'enrichir avec du grain, on sème toujours. Qu'arrive-t'il? Le fumier manque, la terre se lasse et le laboureur se ruine en travaillant....Apprenez-le, tous et chacun, le fumier est la richesse du fermler. Voulez-vous savoir une autre chose, la voilà: La terre ne s'améliore que par le fumage et le labourage; clouez ça dans votre caboche.-Bujault.

Qu'est-ce que blen cultiver? $x^{\circ}$ labourer, $2^{\circ}$ labourer, $3^{\circ}$ fumer. C'est aussi employer de la bonne semence, semer copleusement et enlever toutes les mauvaises herbes quil peuvent pousser pendant la aaison.-Caton, 95-46 a.J.C. 



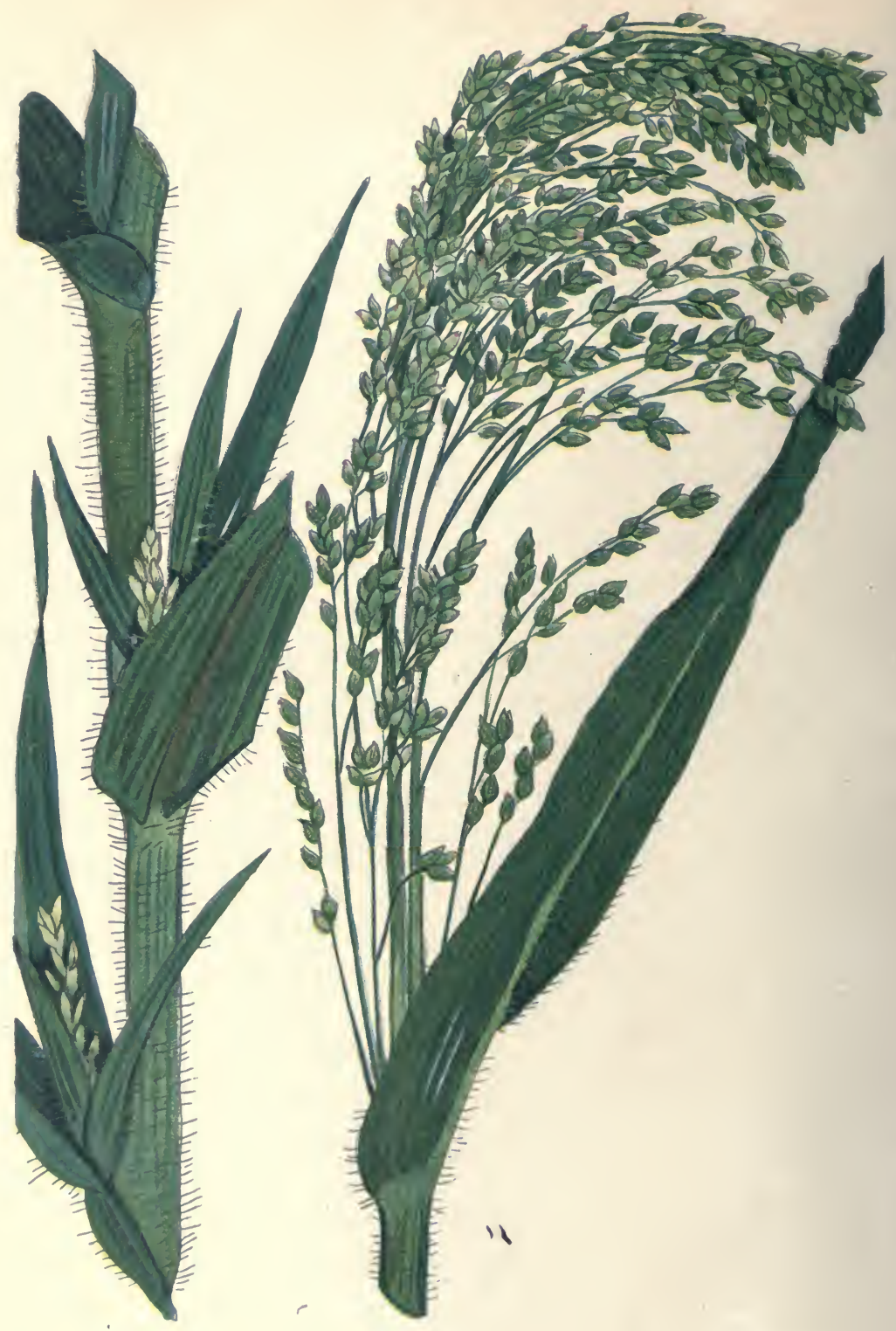

Planche |

MILLET COMMUN

(Panicum miliaceum L.) 


\section{MILLET COMMUN. (Panicum miliaceum L.)*}

Planche I; graine, planche 27 , fig. I.

Description botanique: Le millet commun est une plante annuelle, généralement de deux à quatre pieds de hauteur. Les tiges sont dressées ou ascendantes, naissant d'une base couchée, assez fortes, et couvertes de poils hérissés. Les feuilles, également velues, sont plates et larges. Les fleurs sont portées en grandes panicules, souvent penchées et contiennent un grand nombre d'épillets. Règle générale, ces épillets sont vert vif mais parfois ils ont une teinte noirâtre et pourpre. Chaque épillet contient une seule fleur avec un pistil rouge vif.

Historique: On ne connait pas la plante sauvage d'où descend le millet commun cultivé; sa culture est plus ancienne que l'histoire. Les habitants lacustres de la Suisse le cultivaient 2000 ans avant Jésus-Christ et on en a également retrouvé des traces dans les habitations lacustres de l'Italie. On le cultive dans l'est et le sud de l'Europe. C'est une plante très importante dans l'est et le sud de l'Afrique. Il a été introduit assez tôt en Amérique mais n'a jamais été l'objet d'une grande culture au Canada.

Variétés: De même que toutes les autres plantes depuis longtemps cultivées, le millet commun se divise en un grand nombre de variétés qui diffèrent l'une de l'autre principalement par la forme et la structure de la panicule et la couleur de la graine. Dans quelquesunes, la panicule est ouverte et droite, dans d'autres elle est resserrée et ressemble à un épi. Les millets à balai ont une panicule penchée et étalée qui ressemble à la grappe de graines du maîs à balai. Les noms des autres variétés tels que millet blanc et millet rouge français, se rapportent à la couleur de la graine.

Valeur agricole: Le millet commun est plus propre à l'alimentation de l'homme que tous les autres millets mentionnés. C'est là l'objet principal de sa culture dans l'est de l'ancien continent. Dans le centre de l'Europe il fournissait, avant la découverte de la pomme de terre, l'élément principal de la nourriture de l'homme pauvre. Aujourd'hui, il n'a pour ainsi dire plus d'importance comme plante alimentaire. Dans l'Amérique du nord on l'emploie exclusivement dans la production de fourrages.

*Cette plante est le millet commun d'Europe, qui est cultivé en ce pays depuis un temps immémorial. La plante que l'on appelle parfois le millet commun en Amérique n'est pas le millet commun d'Europe, c'est un millet a grappe, principalement de la variété hongroise au Canada. Ce fait a donné lieu à quelque confusion. 
Le millet dont on se propose de faire du foin doit être coupé juste à l'époque voulue. C'est pendant la floraison qu'il renferme le plus de nourriture; passé cette phase la qualité du foin se détériore rapidement. Semé pour fourrage vert ou pâturage on doit employer trente livres de graine à l'acre; cultivé pour la production de la graine, vingt livres suffisent.

Graine: Les graines du millet commun sont beaucoup plus grosses que celles des millets à grappe. Elles ont environ un-huitième de pouce de long, sont ovales, un peu aplaties; le côté extérieur est plus convexe que l'intérieur, elles sont luisantes et de couleur différente dans les diverses variétés. Les couleurs ordinaires sont blanche, rouge, jaune, brune, grise et noire. La graine du millet japonais, qui est la variété la plus répandue de millet commun au Canada, pèse soixante livres au boisseau.

Mesurez vos entreprises sur vos facultés, de crainte d'être obligé d'abandonner honteusement ce que vous aurez entrepris; un petit terrain bien cultivé est plus productif qu'un grand terrain négligé.Palladius, IVe sièce.

Un nommé Paridius avait deux filles et pour bien une vigne. Lorsqu'il maria l'alnée, il lui donna pour dot le tiers de cette vigne, et, malgré cet abandon, il recueillit, du reste de sa propriété, le même produit que précédemment. En mariant, plus tard, sa seconde fille, il partagea avec elle la vigne qui lui restait. Son revenu annuel n'éprouva aucune diminution par ce nouveau partage, parce qu'il perfectionnait sa culture à mesure qu'il se dessalsissait d'une partie de son fonds.-Columeile, Ier siecie .

Admirez, si vous voulez, une grande ferme mals n'en cultivez qu'une petite.-Virgile, 70-19 a.J.C.

Ne sème que ce que tu peux fumer; fais des prés, élève du bétail jusqu'à ce que tu puisses fumer tous tes blés.-.. Ce n'est pas ce qu'on sème, c'est ce qu'on fume qui produit... Le blé convenablement fumé résiste aux intempéries, tandis que celui qui ne l'est pas se fond comme beurre au soleil; on dirait au printemps que chaque jour il rentre dans la terre.-Plus tu as de fumier, plus tu en auras, plus tu recolteras, plus tu t'enrichiras. Je puis donc dire: L'agriculture est dans un mot.. C'est le pré qui fume le blé; c'est lui quil produit tout, nourrit l'homme et la bête.-Il est encore aisé d'avoir du bétail. mais il faut qu'il vlve... On ne l'envole pas chercher son pain de porte en porte; il est attaché, criant, souffrant et mourant.-Jacques Bujault, $177 x-1842$. 



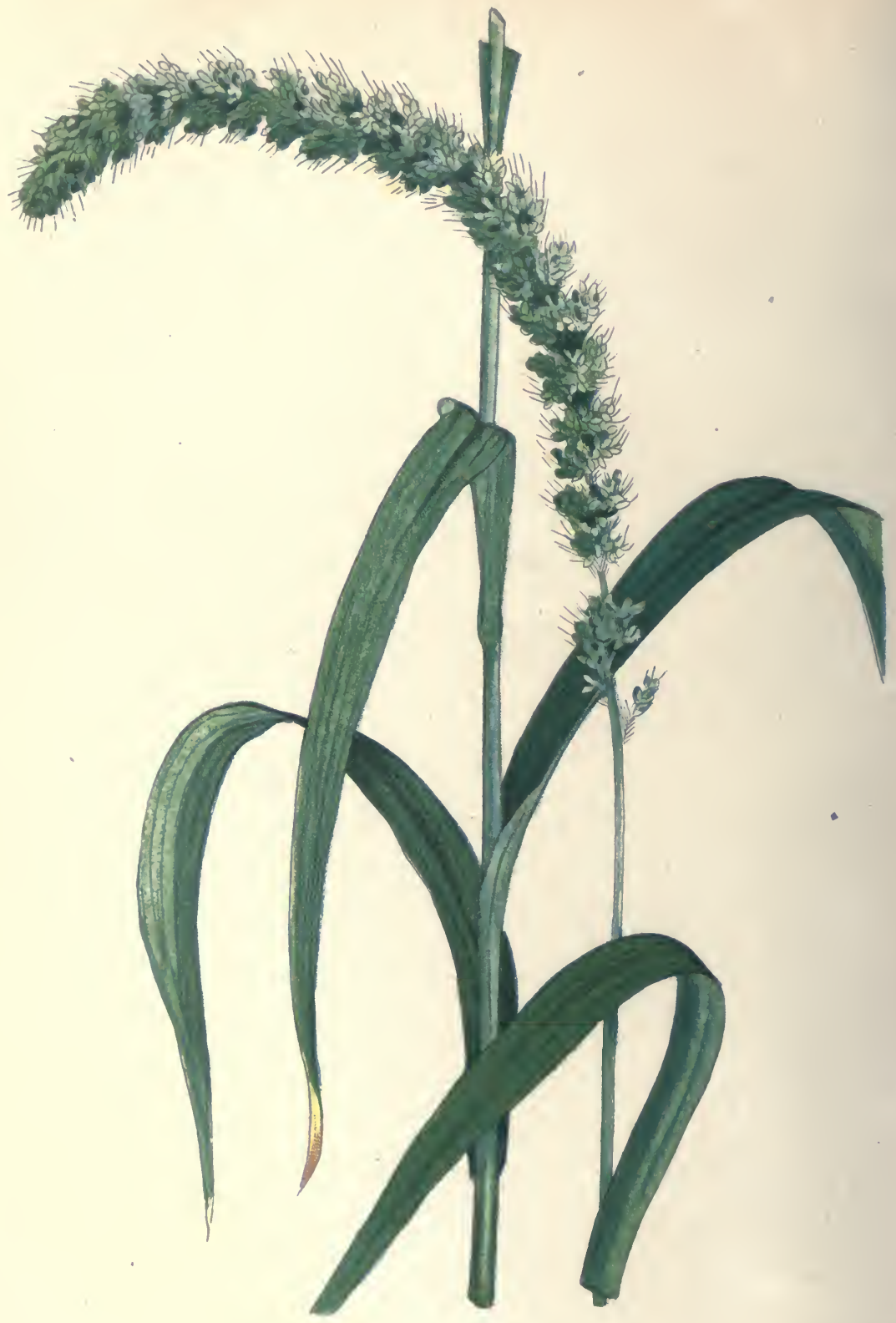

Planche 2

MILLET DE HONGRIE

(Setaria italica (1.) Beaur.) 


\section{MILLET DE HONGRIE OU MILLET À GRAPPE (Setaria italica, (L.) Beauv.) \\ Autre nom latin: Ixophorus italicus (L.) Nash.}

Autres noms français: Millet d'Italie, Panic d'Italie, millet des oiseaux.

Nom anglais: Foxtail millet.

Description botanique: Le millet à grappe est une plante annuelle à tiges assez raides et dressées, généralement de deux à quatre pieds de hauteur, mais atteignant parfois une hauteur de six pieds ou plus. Les feuilles sont larges, assez semblables de forme et de texture à celles du millet commun. Cependant on le distingue facilement de cette dernière plante, même quand il est très jeune, par l'absence de poils. L'inflorescence est une panicule resserrée, souvent penchée au sommet; ses branches courtes lui donnent l'aspect d'un épi. Règle générale les branches basses ne sont pas aussi serrées que les branches supérieures. Les épillets sont serrés et mélangés de poils raides; ces derniers représentent les branches sur lesquelles il ne s'est pas développé d'épillets. Ces poils sont généralement longs, souvent rougeâtres ou pourpres. Ils donnent à la panicule l'aspect d'une queue de renard, et c'est de là que vient le nom anglais "foxtail" des millets cultivés qui appartiennent au genre Setaria. Chaque épillet ne contient qu'une seule fleur à pistil jaune.

Historique: On croit généralement que le millet à grappe descend de la sétaire verte ou mil sauvage (Setaria viridis (L.) Beauv.), une mauvaise herbe gênante dans maintes parties du Canada. Mais les preuves à l'appui de ce fait ne paraissent pas concluantes. Quoiqu'il en soit, sa culture date d'un temps très reculé. C'est une des cinq plantes sacrées qui, d'après un décret promulgué en l'an 2700 avant Jésus-Christ, étaient semées chaque année à une cérémonie publique par l'empereur de Chine. Il est très cultivé actuellement dans l'Asie du Centre, le nord de l'Inde, la Chine et le Japon. On le cultive également dans le sud et l'est de l'Europe, mais il n'a là qu'une importance secondaire.

Variétés: Il existe un grand nombre de variétés de millets à grappe mais la plupart ne sont pas des variétés au sens botanique du mot et ne sont donc pas clairement définies. Le millet allemand est un exemple de ce fait. Cette espèce varie tant dans son aspect et ses habitudes que l'on n'en trouve pour ainsi dire pas de champ uniforme. Le millet de Hongrie, le millet japonais, le millet sibérien, etc., sont des variétés plus ou moins distinctes qui cependant ne poussent pas de façon très uniforme. 
Valeur agricole: Le millet à grappe est une plante alimentaire importante dans bien des parties de l'Asie et particulièrement dans le le nord de la Chine où l'on moud sa semence pour en faire du gruau. En Amérique elle ne sert pas à la nourriture de l'homme. Pour le foin on la coupe de préférence quand la majorité des plantes sont en fleurs car c'est alors que les tiges et les feuilles contiennent le plus de nourriture. Quand les plantes commencent à fleurir, les poils des épis sont encore mous et inoffensifs, mais lorsque la floraison est passée ils deviennent raides, durs, produisent plus ou moins d'irritation dans les voies digestives des animaux et on dit même qu'ils forment dans l'estomac des boules compactes qui causent de graves désordres et parfois amènent la mort. Dans les pâturages, le millet devrait être brouté avant que les épis soient formés. On sème trente livres de graine à l'acre pour prairie ou pâturage; vingt livres suffisent pour une récolte destinée à la production de la graine.

Graine: La grosseur de la graine varie. Elle est toujours plus petite que celle du millet commun mais elle a la même forme générale, quoique le côté interne soit plus plat. La couleur varie avec les variétés, d'orange et jaune à grise et noire. Parfois on rencontre des graines de couleur différente dans la même variété. C'est le cas, par exemple, dans le millet de Hongrie où la graine varie d'un jaune pâle à noir; on trouve dans la même plante et souvent dans le même épis, des graines de couleur très différente. On n'a encore offert aucune explication satisfaisante de ce fait. Ce peut être le résultat des croisements, et alors il correspondrait aux phénomènes semblables observés dans le blé d'Inde.

Un boisseau de graine de millet à grappe pèse 48 livres.

\section{MILLET PERLÊ (Pennisetum typhoideum Rich.)}

Nom anglais: Pearl Millet.

Le millet perlé est une plante annuelle qui atteint une hauteur de six à quinze pieds sur les terrains d'alluvion riches et sous des conditions climatériques favorables. Les tiges sont extrêmement feuillues et les fleurs sont portées en épis denses qui ont souvent quatorze pouces de longueur. La plante ressemble parfois au blé d'Inde, mais elle est moins robuste et plus rameuse.

Le millet perlé est indigène à l'Afrique tropicale où sa culture est tout.aussi importante que celle du blé en Amérique. Il contient un grand nombre de variétés dont aucune ne s'est montrée adaptée au climat canadien. 


\title{
MILleT PATTE DE COQ (Panicum Crus-galli L.)
}

\author{
Graine, Planche 26, fig. 2.
}

\section{Autres noms latins: Echinochloa Crus-galli (L.) Beauv,; Oplismenus Crus-galli Drum.}

Autres noms français; Ergot de coq, patte de poule.

Noms anglais: Barnyard Grass. Barnyard Millet.

Description botanique: Le millet patte de coq est une plante annuelle qui atteint une hauteur de un à trois pieds. Les tiges, souvent genouillées sont ascendantes, plutôt fortes. Il diffère des autres millets par les gaines des feuilles qui sont comprimées et très carénées et par la touffe de poils longs qu'il porte à la base de la feuille. La panicule se compose de nombreux groupes d'épillets portés du même côté; de forme, de couleur et d'aspect général, variables. Chaque épillet consiste en une fleur simple qui, généralement, a une forte barbe courte Dans certaines variétés (par exemple le millet patte de coq japonais) la barbe fait défaut, dans d'autres au contraire elle est très longue.

Distribution géographique: Le millet patte de coq est indigène à l'ancien monde où on le rencontre dans les champs humides, dans les jardins, le long des chemins et des fossés, dans des endroits incultes, etc,. C'est souvent une mauvaise herbe gênante. Il n'est pas indigène au Canada, mais il y a été introduit de bonne heure.

Valeur agricole: Comme ce millet est une herbe grossière qui perd rapidement de sa qualité après avoir fleuri, il devrait être coupé et converti en foin quand les plantes sont en fleurs et même plus tôt. Si on se propose d'en faire de l'ensilage, on peut retarder la coupe jusqu'à ce que la floraison soit bien passée.

Pour le foin on sème 20 livres à l'acre; pour la production de la graine douze livres et demie suffisent.

Tout le monde vous dira qu'il n'y a rien de meilleur que le fumier pour bonifier un champ. On le voit se former de lui-même; on sait comment il se fait; on peut s'en procurer la quantité nécessaire; cependant les uns prennent la peine d'en rassembler, les autres n'y pensent pas. Celui qui règne dans les cieux nous envoie des pluies qui convertissent toutes les fosses en mares. La terre, d'un autre coté, produit toutes sortes de plantes parasites dont on doit la délivrer lorsqu'on veut semer. Une fois arrachées, jetez-les dans l'eau, le temps va les transformer en principes de fécondité. Quelle herbe, en effet, quelle terre ne se convertit pas en fumier dans les eaux stagnantes? -Xénophon, 434-355 a. J. C. 


\section{ALPISTE DES CANARIES. (Phalaris arundinacea L.)}

Semence, planche 26 , figure 4 .

Autre nom français: Millet long.

Nom anglais: Reed Canary Grass.

Description botanique: L'alpiste est une plante vivace à souche traçante et vigoureuse d'où naissent des rhizomes ou stolons souterrains, longs, et écailleux. Ces rhizomes s'allongent beaucoup et produisent plus tard des tiges lisses et fortes, de deux à six pieds de hauteur. Les feuilles sont larges, d'un pied de longueur ou à peu près, et parfois marquées de raies blanches. La panicule est forte, à rameaux plutôt courts, étalés pendant la floraison mais se redressant plus tard. Les épillets, qui sont serrés vers l'extrémité des branches, sont étroits, d'un vert pâle, parfois légèrement teintés de pourpre. Ils sont également un peu courbés et ne contiennent qu'une fleur, sans barbe. La panicule ressemble à celle du dactyle pelotonné mais on la distingue facilement de cette dernière par ses épillets à une fleur.

Distribution géographique: L'alpiste est indigène à presque toute l'Europe et aux parties tempérées de l'Asie, de la Sibérie et de l'Amérique du nord. Il est assez répandu au Canada surtout dans les provinces des prairies.

Habitat: Il croît spontanément dans les terrains bas et humides, le long des cours d'eau et des fossés, dans les marais et les marécages. Malgré sa préférence pour les terrains humides, il endure de longues sécheresses. La gelée l'affecte peu.

Valeur agricole: Cette herbe devient plutôt raide et coriace en vieillissant et il faut l'utiliser comme foin ou comme pâturage quand elle est relativement jeune. Dans bien des parties des grandes plaines des Etats-Unis, elle forme une proportion considérable du foin indigène.

La terre enseigne d'elle-même la justice aux ames attentives, car elle comble de blenfaits ceux quil la comblent de soins,-Xénophon, 434-355 a.J.C. 


\section{FLOUVE ODORANTE. (Anthoxanthum odoratum L.)}

Semence, planche 26 , figure 5 .

Description botanique: La flouve odorante est une piante vivace à souche courte et à tiges touffues. Les tiges atteignent une hauteur de six pouces à deux pieds et les feuilles sont portées principalement vers la base. Ces feuilles sont vert vif, courtes et velues le long des bords, surtout en dessous. Les fleurs sont en panicule épaisse, cylindrique, verte quand elle est jeune mais qui, plus tard, devient jaune d'or. Chaque épillet contient trois fleurs dont deux sont stériles et très réduites. Chaque fleur stérile se compose d'une glume foncée, couverte de poils épais et raides et munie d'une barbe forte, genouillée. La fleur fertile, qui est placée entre les deux stériles, est du type ordinaire mais elle ne contient que deux étamines.

Distribution géographique: La flouve odorante se rencontre sur de grandes superficies de l'ancien monde. Elle est répandue dans la plupart des pays européens, l'ouest et le nord de l'Asie et certaines parties du nord de l'Afrique. Elle a été introduite dans l'Amérique du nord et se rencontre particulièrement dans l'est du Canada.

Habitat: Elle croît spontanément dans les prairies, les bois, les jardins et sur presque toutes sortes de sol. Elle préfère cependant les terrains frais, sablonneux ou argilo-sableux, mais la sécheresse l'affecte peu.

Valeur agricole: La flouve odorante est une des herbes les plus précoces, mais elle a peu de valeur comme plante fourragère à cause de sa végétation basse et de ses feuilles courtes. Elle contient une substance odorante qui communique au foin une odeur agréable mais en même temps un goût amer. Les bestiaux n'en sont pas friands.

Si tu te moques de la terre, elle se moquera de toi. Pour qu'elle rende, il faut lui prêter; elle ne donne rien pour rien.-Bujault. 
MUHLENBERGE. (Muhlenbergia racemosa (Michx). BSP.)

Semence, planche 26, figure 6 .

Autre nom latin: Muhlenbergia glomerata Trin.

Nom anglais: Knot root grass.

Description botanique: La Muhlenberge est une plante vivace à racines vigoureuses. Ses souches traçantes et ramifiées sont irrégulièrement recouvertes de tubercules et émettent de nombreux stolons écailleux, de l'extrémité desquels naissent les tiges. Les tiges, de un à trois pieds de hauteur, assez succulentes quand elles sont jeunes, deviennent dures et coriaces en vieillissant. Elles portent de nombreuses branches, surtout à la base, et forment des touffes lâches ou des tapis assez serrés et étendus. Les feuilles sont étroites, nombreuses et serrées, principalement à la base des tiges. Les panicules sont resserrées, de trois à quatre pouces de longueur, à rameaux courts et étroits. Ces derniers sont recouverts d'un grand nombre d'épillets étroits qui se composent de deux glumes stériles, en forme d'alène, enveloppant une seule fleur plus courte que les glumes.

Distribution géographique et habitat: La muhlenberge est indigène au Canada, elle est répandue sur presque toute l'étendue du pays. Elle atteint son plein développement sur les sols sablonneux ou graveleux et ne pousse pas sur les sols trop humides. Dans ces derniers les tiges sont basses et toute la plante est souvent teintée de pourpre.

Valeur agricole: Cette plante a été pendant quelque temps l'objet d'essais de culture mais sa valeur agricole n'est pas encore bien établie. Quand on la cultive pour la formation des prairies ou des pâtures on devrait semer environ vingt livres de bonne graine à l'acre.

Tout ce qu'on a à faire en agriculture doit être bien fait du premier coup; car, lorsque, par négligence ou par imprudence, il faut recommencer ce qui est mal fait, le temps convenable est passé et rien ne peut en réparer la perte, ni balancer les avantages qu'on aurait obtenus en profitant du moment opportun.-Pline, Histoire naturelle 23-79, A.D. 



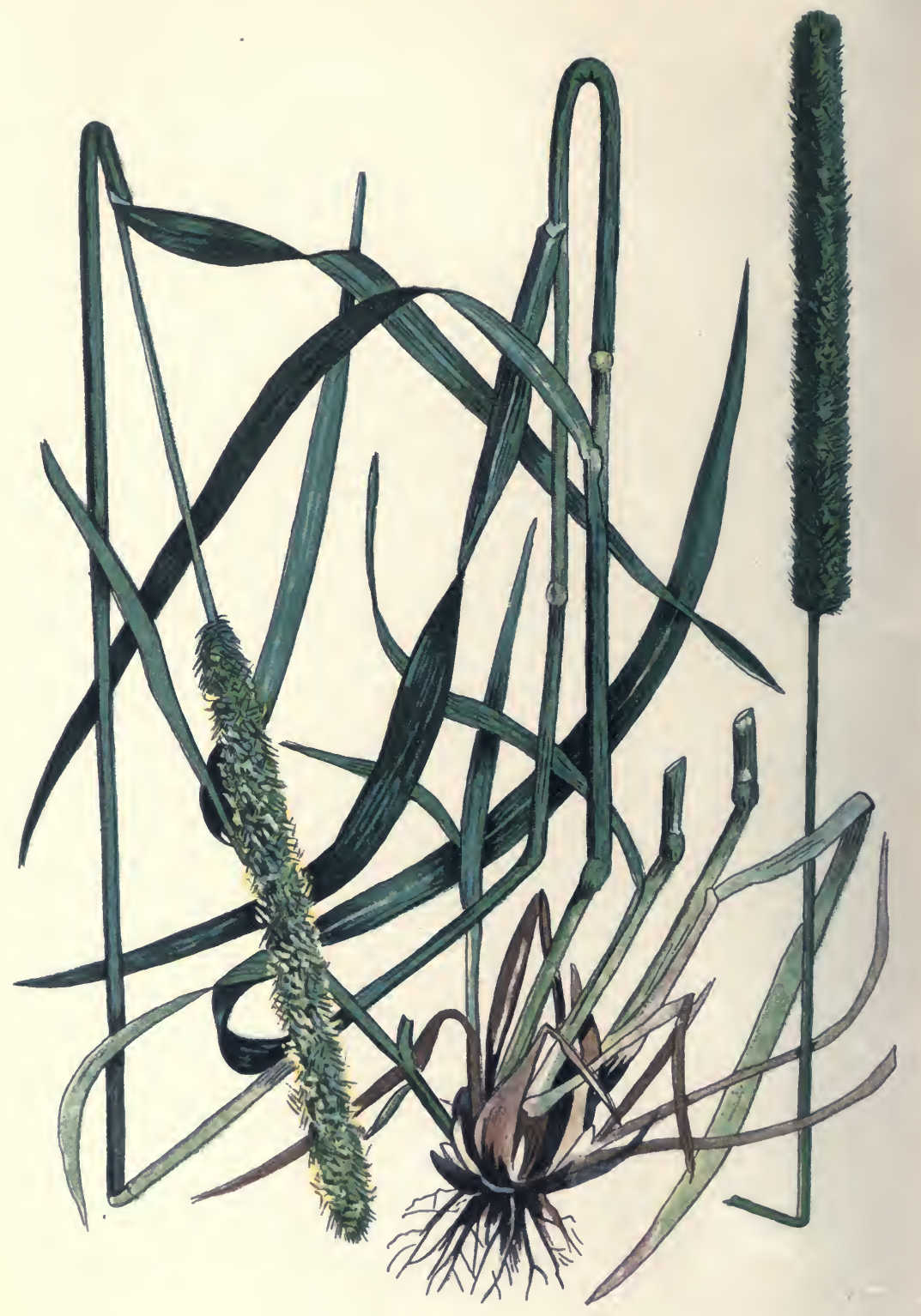

Planche 3

FLÉOLE DES PRÉS OU MIL (Phleum pratense L.) 


\section{MIL ou FLÉOLE DES PRÉS (Phleum pratense L.)}

Planche 3 ; semence, planche 26 , fig. 7 .

Noms anglais: Timothy, Meadow Cat's Tail, Herd's Grass.

Description botanique: Le mil est une herbe vivace, à souche très courte, et qui, en raison de ce fait, pousse en touffes plus ou moins compactes. Les tiges, qui atteignent une hauteur de un à quatre pieds ou plus, sont lisses et généralement dressées. Elles s'épaississent à la base, surtout sur les terrains secs et durs, en une sorte de bulbe qui contient une provision de nourriture d'un genre particuiier. Les feuilles qui sont enroulées dans le bourgeon, sont généralement courtes par comparaison à la hauteur de la plante. Les épillets sont arrangés en épi cylindrique, épais; chaque épillet ne contient qu'une fleur renfermée dans une paire de glumes fortement carénées, qui ne sont pas attachées ensemble comme dans le millet à grappe. Au point de vue de la forme et de la grosseur les épis du mil et du vulpin des prés sont assez semblables, mais ceux du mil sont rudes au toucher tandis que l'épi du vulpin des prés est très doux. Les organes mâles et femelles dans la fleur, sont arrangés de façon à encourager la fécondation croisée qui est effectuée par les vents.

Distribution géographique: La fléole des prés est indigène à l'Europe, à l'exception de la Turquie. Elle est également indigène au nord de l'Afrique et à de vastes superficies de l'ouest et de l'est de la Sibérie. Elle a été introduite par les premiers colons dans le nord de l'Amérique et sa culture est maintenant assez répandue dans tout le nord des Etats-Unis et l'Ouest Canadien.

Historique: Quoique la fléole des prés soit indigène à l'Europe, c'est en Amérique que l'on a commencé à en apprécier la valeur. Elle fut importée dans le Maryland vers I 720 par Timothy Hanson, qui lui a donné son nom anglais. Dans la Nouvelle-Angleterre elle est connue sous le nom de Herd Grass. Ce serait, paraît-il, un nommé Herd qui la trouva dans le New-Hampshire et l'introduisit dans la culture. Au Canada elle est mieux connue sous le nom de mil.

Culture: Dans les terrains froids, humides ou marécageux et particulièrement dans les argiles lourdes, le mil n'a pas d'égal pour la production du foin. C'est sur les terrains frais, argileux ou argilo$28550-5$ 
sableux qu'il réussit le mieux. Il ne vient pas bien sur les terrains acides ou exposés à se dessécher pendant les chaleurs tels que les sables appauvris ou les terrains maigres sur fonds de roches.

Le mil est essentiellement une plante à climats tempérés; l'humidité est, pour lui, un facteur plus important que la température. Il résiste très bien au froid et supporte longtemps une couche épaisse de neige. Il supporte assez bien la sécheresse, bien que ses racines soient peu profondes, mais il rend peu dans un sol trop sec.

Variétés: Le mil comprend des types innombrables, très différents l'un de l'autre et très différents également en valeur agricole. Dans les endroits où il a échappé à la culture et où il est redevenu sauvage on trouve des centaines de types poussant côte à côte, dans des conditions exactement semblables. On voit des plantes géantes, extrêmement feuillues, et, par conséquent, d'une grande valeur économique, poussant tout à côté de types petits et nains, à feuilles rares, dont les épis n'ont qu'un demi pouce de long; on voit des touffes ouvertes, à tiges ascendantes ou presque couchées poussant en compagnie de touffes épaisses et très garnies. On trouve des plantes d'un vert pâle, vert bleuâtre et rouge bleuâtre, poussant côte à côte. De même, des types hâtifs, où les feuilles de la base sont brunes et mortes, se rencontrent à côté de plantes à maturation tardive, portant quantité de feuilles vertes.

Habitudes: La fléole des prés pousse assez lentement; règle générale sa maturation est de moyenne à tardive. Elle fleurit au commencement de juillet dans la péninsule sud-ouest de la province d'Ontario et du milieu à la fin de juillet au Manitoba et au nord-est de Québec. La graine arrive à maturité environ un mois après la floraison. Semée avec une céréale, au printemps, elle donne une bonne récolte de foin l'année suivante.

Valeur agricole: Le mil est cultivé au Canada presque à l'exclusion de toutes les autres herbes. La raison principale de cette préférence est que la graine est généralement peu coûteuse, propre et de bonne faculté germinative. Les semis de mil reviennent moins cher à l'acre que ceux de toute autre herbe fourragère.

Le mil donné seul n'a qu'une faible valeur nutritive pour les animaux en état de croissance ou pour ceux qui donnent du lait, car il manque de ces éléments qui forment la chair. Ce n'est donc pas un fourrage avantageux pour la production de la viande ou du lait. 
L'addition de trètle l'améliore. On le recherche pour les chevaux de travail qui reçoivent également de fortes rations de grain, car il est très digestif. C'est le foin régulier pour tous les chevaux d'attelage dont on exige un travail immédiat après le repas.

Semé seul, le mil ne donne pas une récolte bien épaisse, sauf sur les terres riches et humides; sur les terres hautes il vaut mieux le semer en mélange avec d'autres herbes ou avec du trèfle rouge. Quand l'assolement est de courte durée et que la prairie est relevée tous les deux ans, on peut, sur des terrains lourds, frais ou humides, le semer seul ou avec du trèfle d'alsike; sur des terrains plus secs et plus légers on le sème avec du trèfle rouge. Semé un peu épais, il donne une herbe plus fine qui doit être coupée peu après la formation de l'épi et l'apparition des premières fleurs. On obtiendrait un rendement plus élevé si on retardait la coupe jusque vers la fin de la floraison, mais le foin y perdrait en quantité et en valeur nutritive. Quand elle a atteint tout son développement la tige devient dure et coriace. Si l'on désire avoir une seconde pousse, il faut couper juste avant la floraison, le regain en sera plus abondant.

Quand on sème le mil seul on doit appliquer de neuf à quinze livres de bonne graine fraîche à l'acre.

Le mil ne convient pas pour les pâturages, sauf en mélange avec d'autres herbes. Ses racines peu profondes et sa croissance en touffes ne lui permettent pas de résister au piétinement aussi bien que les autres plantes généralement recommandées pour le pâturage. Dans les terres hautes et légères il cède bientôt la place aux herbes indigènes, surtout si ces terres sont abandonnées aux moutons qui paissent au ras du sol.

Graine: Le mil semé pour la production de la graine doit être récolté dès que la plante a atteint sa maturité, complète lorsque l'épi, vert jusque-là, devient jaunâtre. Récoltée trop tôt la graine est petite, peu développée et germe mal. Récoltée quand elle est mûre elle est exposée à perdre sa balle au battage, elle perd en même temps son éclat argenté et prend l'aspect terne de la vieille graine.

On bat généralement le mil avec une batteuse à grain ordinaire mais pour obtenir la meilleure semence il faut le récolter à la main et le battre au fléau. On le cultive dans la vallée du Saint-Laurent et les districts de la Baie Georgienne et la qualité de la graine, au point de vue de la grosseur et de l'éclat de la couleur, n'est pas surpassée. On sépare parfois sa graine des criblures du blé d'automne qui a été semé sur gazon de mil, mais cette graine est généralement in- 
festée de lin sauvage et d'autres graines de mauvaises herbes communes aux herbages.

La graine de bonne qualité est de couleur argent vif; une petite partie seulement est écalée, c'est-à-dire, a perdu sa balle. La semence est terne lorsqu'elle est vieille ou lorsqu'elle a été récoltée ou emmagasinée dans de mauvaises conditions. Les semences écalées qui viennent d'être battues ont tout autant de vitalité que les autres mais elle la perdent plus vite que celles qui ont conservé leurs balles. La graine bien mûre, conservée dans un endroit frais et sec, conserve sa vitalité de trois à cinq ans. Des graines de neuf ans même, donnent une forte proportion totale de graines viables quoique généralement, à cet âge, le germe est sensiblement affaibli.

Le poids légal du boisseau est de quarante-huit livres.

La grande masse de la graine de mil du commerce est propre par comparaison aux graines des autres graminées et de trèfles. Les graines de mauvaises herbes contre lesquelles on doit principalement se garder quand on fait des achats de graine de mil sont les suivantes: grande marguerite, cameline, camomille puante, oseille de brebis, silène enflée, laiteron vivace, chardon du Canada, mouron et potentille.

Le mil, comme beaucoup d'autres espèces de graminées, est attaqué par l'ergot (Claviceps). Les graines d'ergot (sclérotes) varient en forme et en grosseur suivant l'espèce de grain ou d'herbe sur laquelle ils se développent. Les corps solides sont pourpre foncé, on les voit sans peine sortant de l'enveloppe de la graine dans l'épi. Il ne faut pas récolter la graine de prairies infestées d'ergot.

Tu laisseras une année sur deux, après moisson faite, la terre rester en friche, et le champ inactif puiser dans le repos des forces nouvelles; ou bien encore tu sèmeras, à la saison prochaine, le froment doré, dans le même champ où tu auras d'abord récolté en abondance maint légume à la silique tremblante, les cosses grêles de la vesce, ou l'amer lupin dont les tiges fragiles bruissent en touffes sonores. Sans doute, la graine du lin brâle la terre; II en est de même de l'avoine, de même aussil des pavots, qui portent en eux le sommeil du Léthé. Mals pourtant, avec ces alternances, le laboureur est payé de sa peine; seulement, qu'il ne craigne pas, dans un sol aride, de répandre à profusion l'engrais et le fumier, nl de jeter des tas de cendres sur les champs épulsés. C'est ainsi qu'en changeant de production la terre se repose, et que le sol, pour être épargné, ne reste pas, cependant, stérile.-Virgile, Les Géorgiques, Traduction d'Henri Lantoine.

Lé pré se fatigue à la longue et demande à être renouvelé de temps à autre par une plantation de haricots, de navette ou de millet, que l'on remplace l'année suivante par du blé et la trolsième année par du foln.-P!ñe, Histoire naturelle, 23-79 A. D. 



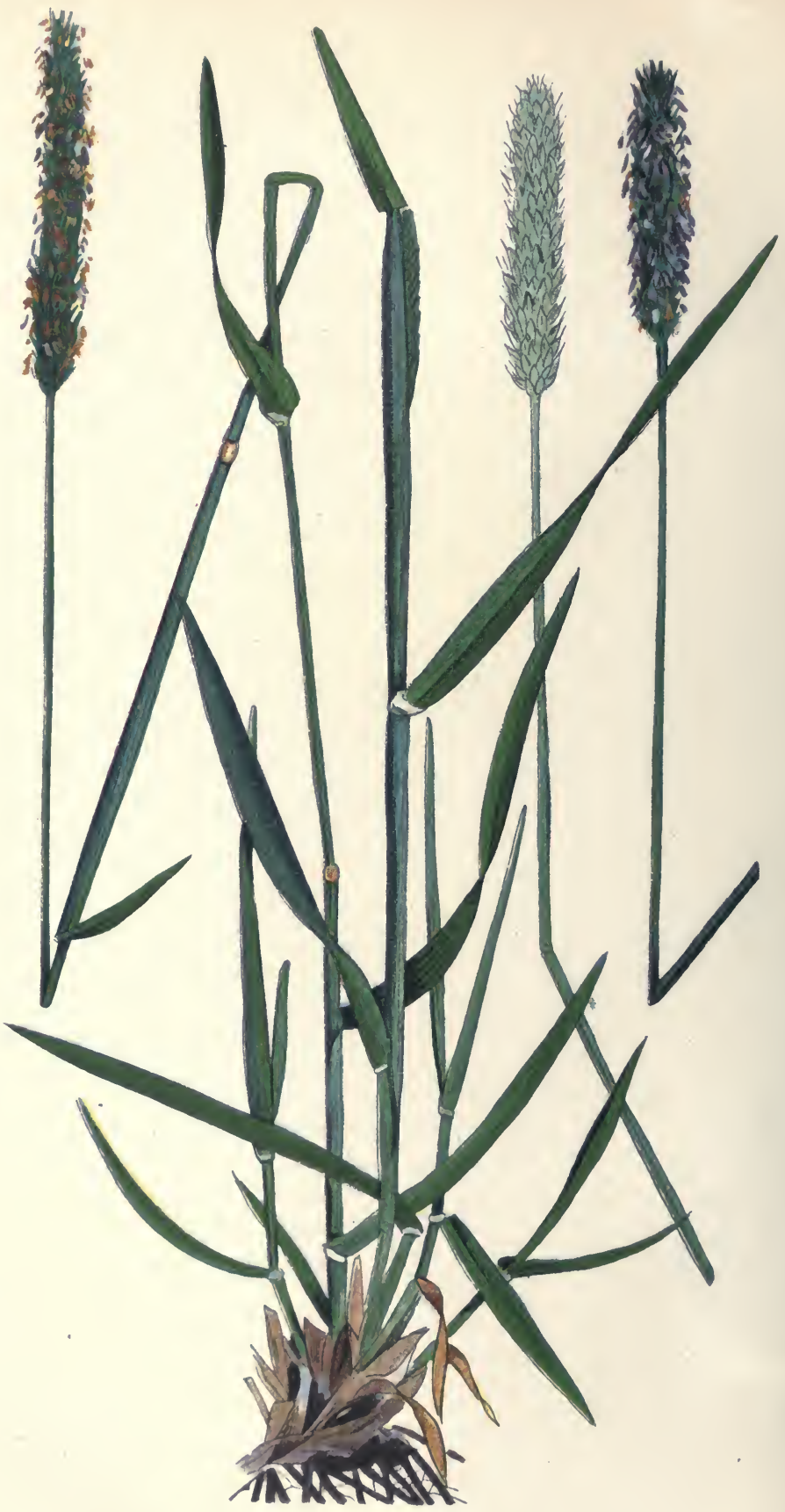

Planche 4.

VULPIN DES PRÉS

(Alopecurus pratensis 1 .) 


\section{VULPIN DES PRÉS. (Alopecurus pratensis L.)}

Planche 4 ; graine, planche 26, fig. 8 .

Autres noms français: Chiendent queue de renard, vulpine.

Nom anglais: Meadow Foxtail.

Description botanique: Le vulpin des prés est une plante vivace qui ressemble beaucoup au mil. Il a une souche courte qui produit des rhizomes écailleux. Aux extrémités de ces rhizomes naissent des tiges et des rejetons feuillés. Si les rhizomes sont très courts, ce qui se produit généralement dans les terrains relativement secs, la plante entière pousse en touffes, presque comme le mil. $\mathrm{Si}$, au contraire, ils atteignent une longueur considérable, comme ils font souvent dans les terrains ouverts et frais, les touffes sont plus lâches et moins apparentes. Les tiges, qui ont de deux à quatre pieds de hauteur, sont parfois genouillées et enracinées à la base. Elles sont lisses et feuillées jusqu'à un point au-dessus du milieu. La majorité des feuilles sont produites par les rejetons de la base; elles sont généralement longues, larges et molles, les gaines des feuilles supérieures sont souvent gonflées.

Les fleurs sont en épi, assez semblable à celui du mil (fléole des prés) mais tandis que l'épi du vulpin des prés, dont les épillets sont recouverts de poils longs et soyeux, est doux au toucher, celui du mil est rude. Cette circonstance permet de distinguer ces plantes l'une de l'autre. Chaque épillet dans le vulpin des prés contient une seule fleur entourée de deux glumes à carène aiguë attachées ensembles à leur base. La fleur porte au dos une barbe qui fait saillie au-dessus du sommet des épillets et donne à l'épi un aspect hérissé. La fécondation est accomplie par les vents; la fleur peut se féconder elle même ou être fécondée par une autre fleur. Ce dernier mode de fécondation, appelé fécondation croisée, est plus commun à cause de la disposition des étamines et du pistil pendant la floraison.

Distribution géographique: Le vulpin des prés est indigène à la plus grande partie de l'Europe, au nord de l'Afrique, au centre et au nord de l'Asie. Il est répandu dans tout l'est et le centre du Canada et on le cultive actuellement dans presque tous les pays de l'Europe. Il croît spontanément dans les prairies humides, les marécages, le long des fossés et des cours d'eau à rives basses, et généralement dans les sols frais et fertiles. 
Culture: Le vulpin des prés préfère les localités humides mais il redoute l'excès d'eau. Il ne vient pas bien dans les champs recouverts d'eau stagnante pendant la plus grande partie de l'année. C'est dans les fonds d'argile et de terre franche, temporairement inondés, qu'il se développe le mieux. Il est extrêmement résistant à la gelée et peut être considéré comme l'herbe la plus hâtive dans l'est du Canada. Il se met à pousser de bonne heure au printemps et atteint son plein développement avant que la plupart des autres herbes aient fait une croissance appréciable. Il est utile partout où l'on désire des pâturages ou du foin de bonne heure.

Valeur agricole: Le vulpin des prés cultivé pour la production du foin doit être coupé pendant qu'il est en fleurs. Les tiges renferment alors une grande quantité de sucre et le foin est doux et nourrissant. Une fois la floraison passée, ce sucre concourt à la formation de la graine et la valeur nutritive du foin diminue rapidement. Cultivé en pâturage, le vulpin des prés fournit une quantité d'excellent fourrage au début de la saison, quand les animaux n'ont encore rien d'autre chose à paître. Il est recherché de tous les bestiaux. C'est évidemment l'une des herbes les plus précieuses sur les terres qui lui conviennent. On le cultive presque toujours en mélange.

Graine: La graine du vulpin des prés mûrit très vite mais assez irrégulièrement, ce qui en rend la récolte assez difficile. Dans bien des parties de l'Europe on la cueille à la main puis on la fait sécher dans un endroit aéré en la retournant tous les jours pendant deux semaines. Les graines qui ne sont pas traitées de cette façon ont une germination assez faible. La graine de commerce a généralement une faible vitalité à cause de cette maturation inégale. Pour obtenir une forte quantité de bonne graine il faut couper la récolte un peu avant qu'elle arrive à maturité complète, faire de petites gerbes, les mettre neuf ou dix ensemble en veillottes rondes et les laisser mûrir. On sème de vingt à vingt-cinq livres à l'acre quand on cultive le vulpin seul.

La bonne graine est couleur de paille et pèse de six à douze livres au boisseau.

On a dit une grande vérité, que l'agriculture est la mère et la nourrice des autres arts. Est-elle en vigueur, tout fleurit avec elle; mais partout ou la terre est condamnée à la stérilité, les arts meurent je diraís presque et sur terre et sur mer.-Xénophon. 434-355 a.J.C. 





Planche 5

AGROSTIDE COMMUNE (Agrostis alba $L$. ) 


\section{AGROSTIDE COMMUNE OU STOLONIFÈRE (Agrostis stolonifera L.)}

Planche 5,: graine, planche 26, fig. 9.

Autre nom latin: Agrostis alba L.

Autres noms français: Agrostide traçante, trainasse, fiorine, agrostide blanche.

Noms anglais: Fiorin Grass, Creeping Bent-grass, White Bentgrass, Red Top.

Description botanique: Le terme agrostide a été appliqué à deux espèces du genre $A$ grostis: $A$. stolonifera L., qui est appelée également $A$. alba $\mathrm{L}$., et $A$. vulgaris With. A. stolonifera et $A$. vulgaris sont deux espèces distinctes mais souvent prises l'une pour l'autre. Les écrivains américains et canadiens ont souvent décrit des plantes d'agrostide sous le nom d' $A$. vulgaris qui est relativement rare en Amérique et n'a qu'une faible valeur agricole. Comme la description dans ces cas n'est pas celle de l'A. vvlgaris mais évidemment celle de l'A. stolonifera, la dernière espèce doit être considérée comme la variété agrostide. La description qui suit se rapporte donc à l' $A$. stolonifera qui est répandue dans toute l'Amérique du nord.

Il existe un grand nombre de variétés d'agrostides très différentes l'une de l'autre. C'est une plante très vivace, à souche traçante, d'où naissent généralement des stolons. Ces stolons sont parfois souterrains, mais généralement ils se développent le long de la surface, émettant des racines aux jointures ainsi que de nombreux rejetons feuillés. A cause de sa souche et des stolons traçants, l'agrostide ne pousse pas en touffes mais elle forme un gazon épais et ininterrompu. Les tiges varient dans les différentes variétés. Dans quelques-unes elles n'ont que deux pouces de hauteur; dans d'autres elles atteignent quatre pieds ou plus. Il n'y a que les variétés de ce dernier type qui soient importantes au point de vue agricole. Les feuilles varient en grosseur, nombre et couleur. La ligule est toujours longue et généralement aiguë. Les fleurs sont arrangées en panicules, à nombreuses branches. A la floraison les branches principales de la panicule ainsi que les branches secondaires sont étalées, et comme leur longueur diminue graduellement vers le sommet de la panicule, cette dernière a la forme d'une pyramide à large base. Lorsque la floraison est terminée, les branches secondaires sont serrées contre les branches principales et ces dernières contre la tige-mère. Après la floraison la panicule est donc souvent étroite et resserrée. Les nombreux 
épillets sont souvent d'un brun rougeâtre, d'où le nom anglais de "Red Top". Chaque épillet ne renferme qu'une seule fleur. A la floraison les pistils et les étamines se développent ensemble et les fleurs peuvent se féconder d'elles-mêmes ou être fécondées par d'autres.

Distribution géographique: L'agrostide est indigène à tous les pays d'Europe, au nord de l'Afrique, au nord et au centre de l'Asie et à l'Amérique du Nord. On a commencé à la cultiver en Angleterre il y a environ deux cent cinquante ans, mais ce n'est que depuis le millieu du dernier siècle qu'elle est généralement cultivée en Europe.

Gulture: L'agrostide croît spontanément dans toutes les localités. Quelques variétés se maintiennent dans les sols légers et sablonneux, ne renfermant que peu d'humidité, mais elles poussent mal et n'ont que peu de valeur agricole. D'autres variétés font une pousse superbe dans les endroits humides et ont une grande valeur. Comme la végétation dépend presque entièrement du développement des racines traçantes, l'agrostide vient mieux dans les sols où rien ne s'oppose à ce développement des racines, comme dans les sols légers et humides tandis que dans les terrains secs et lourds, les souches et les stolons restent courts et peu productifs. L'agrostide a une superbe végétation dans les climats humides. Elle convient donc aux terrains bas, peu éloignés de la mer. Elle est très résistante au froid.

Végétation: Dans les sols convenables, l'agrostide fait une bonne pousse l'année même où elle est semée. Elle se met à croître relativement tard au printemps mais une fois qu'elle a commencé elle continue sans arrêt jusqu'à la fin de l'automne.

Valeur agricole: En mélange avec d'autres espèces dans une prairie, l'agrostide fait une herbe de pied superbe et pousse dans les endroits trop humides pour la plupart des autres graminées. Comme elle se met à pousser assez tard, elle n'a pas généralement atteint son plein développement quand les autres herbes du mélange sont prêtes à être coupées. D'autre part, elle produit des feuilles et des tiges jusqu'à une époque avancée de l'automne et elle est précieuse lorsqu'on désire avoir un regain dans les pâturages. Tous les bestiaux en sont friands,elle résiste très bien au piétinement et même n'en produit qu'un plus grand nombre de rhizomes et de stolons. Elle forme rapidement 
un gazon dense et égal, mais si on la laissait pousser trop longtemps dans un endroit, on pourrait avoir des difficultés à l'extirper. Elle est précieuse pour la formation de pelouses; cultivée seule, il faut semer vingt livres de bonne graine à l'acre.

Graine: Cultivée pour la graine, l'agrostide devrait être récoltée dès que l'on peut faire sortir aisément les graines par le frottement. Règle générale, l'agrostide de commerce contient une grande quantité de balle. La semence que l'on dit être renettoyée n'est autre chose que la graine ordinaire dont on a enlevé une partie de la balle. La graine ordinaire de commerce est d'un brun-rougeâtre avec un lustre argenté, plus ce lustre est argenté, moins il y a de balle et plus la graine est lourde. Quand la proportion de balle est considérable, le poids est assez faible, et parfois il ne dépasse pas huit livres au boisseau. La graine renettoyée, contenant peu de balle peut peser jusqu'à trente-cinq livres au boisseau.

Règle générale, l'agrostide germe bien car la graine conserve sa vitalité plusieurs années.

\section{AGROSTIDE VULGAIRE (Agrostis vulgaris With.)}

Cette herbe a souvent été prise pour la précédente; elle diffère de l'agrostide stolonifère par les traits essentiels suivants:

Elle pousse en touffes, car ses souches sont très courtes et ne produisent pas de stolons. Tous les rameaux de la panicule, le principal aussi bien que les secondaires, s'étalent après la floraison, et comme les rameaux bas principaux sont généralement un peu plus courts que ceux du milieu, la panicule ressemble plutôt à un oeuf qu'à une pyramide. La ligule est extrêmement courte et parfois absente.

Distribution géographique: Cette plante a la même distribution géographique dans l'ancien monde que l'agrostide stolonifère; il est douteux qu'elle soit indigène à l'Amérique du Nord.

Habitat: Elle crôit spontanément dans les sols sableux ou graveleux et résiste mieux à la sécheresse que l'agrostide stolonifère.

Valeur agricole: Il existe à peu près la même relation entre la valeur agricole de l'Agrostis vulgaris et celle de l'agrostide stolonifère 28550-6 
qu'entre la valeur de la fétuque ovine et de la fétuque des prés. En d'autres termes, l'agrostide commune est une herbe plutôt inférieure, que l'on doit laisser de côté chaque fois que l'on peut utiliser des herbes plus précieuses. Les feuilles et les tiges sont assez courtes, les premières sont généralement serrées près de terre; elle ne fait pas un foin avantageux. Sa principale valeur serait comme herbe de pâturage sur un sol pauvre et sec.

Graine: La graine est semblable à celle de l'agrostide stolonifère mais elle est en général un peu plus petite et plus jaunâtre. Dans certains cas, cependant, il est presque impossible de séparer les graines des deux espèces.

\section{GALAMAGROSTIDE DU GANADA (Calamagrostis canadensis Michx. Beauv.)}

Graine, planche 26, figure Io.

Autre nom latin: Deyeuxia canadensis Hook.

Noms anglais: Small reed grass, Sand grass, Canada Bent-Grass, Blue Joint Grass.

Description botanique: La Calamagrostide est vivace, à souche traçante d'où naissent des rhizomes écailleux, bruns. Ces rhizomes indiquent que la plante ne pousse pas en touffes serrées. Les tiges sont plus ou moins groupées, suivant le caractère du sol. Elles ont de trois à cinq pieds de hauteur sont plutôt fermes de texture et d'un brun-rougeâtre ou rouge bleuâtre à la base. C'est pourquoi on appelle cette plante Blue Joint Grass en anglais. Les feuilles sont nombreuses, larges, longues et très rudes. Les fleurs sont portées en une grande panicule, semblable à celle de l'agrostide stolonifère, et généralement d'un brun-rougeâtre. C'est à cause de cela que l'on appelle cette plante"agrostide"dans bien des parties de l'est du Canada, mais à tort. Quoique la panicule soit semblable, les différences entre les deux espèces sont nettement marquées. La façon la plus facile et la plus sûre de les identifier est d'examiner les fleurs. La calamagrostide n'a qu'une fleur par épillet, tout comme l'agrostide, mais cette fleur 
a une barbe qui est entourée à la base d'une touffe de poils blancs et soyeux, très distincts et à peu près de la longueur de la fleur ellemême. Ces poils ne se trouvent jamais à la base de la fleur de l'agrostide.

Distribution géographique: La calamagrostide est propre au Canada et aux parties du nord des Etats-Unis.

Habitat: Elle croît spontanément dans les prairies humides, les marécages, le long des rivières et des ruisseaux, sur les rives des lacs et généralement sur les fonds humides.

Valeur agricole: Parfois elle couvre de grandes superficies, à l'exclusion de toutes les autres herbes. On dit que le foin ramassé sur ces superficies est de qualité excellente et recherché de toutes les sortes de bétail. On sait également qu'il reste savoureux et nourrissant longtemps après avoir fleuri. Il y aurait des expériences à faire pour confirmer cet avancé, mais rien ne nous autorise à le mettre en doute et du reste il y a des preuves à l'appui. On a essayé, mais sans succès, de faire venir la calamagrostide de semis à l'une des stations expérimentales des Etats-Unis; la graine paraissait manquer de vitalité. Ceci peut signifier que la plante ne forme pas de graines ou n'en forme que très peu, comme dans le cas du roseau commun (Phragmiles communis Trin.). S'il en est ainsi, il est facile de comprendre pourquoi cette herbe conserve ses qualités nutritives après la floraison. Comme nous l'avons fait remarquer dans la description du vulpin des prés, les éléments nutritifs concourent à la formation de la graine, mais lorsqu'il ne se forme pas de graines, ils restent dans le foin qui conserve ainsi sa qualité même après que la plante a fleuri.

En fait, on sait peu de chose sur la valeur nutritive de la calamagrostide. Il peut se faire que ce soit une addition importante aux plantes fourragères canadiennes, mais nous ne pouvons rien dire de positif sur ce sujet actuellement. Elle est utile pour les sols très frais car elle pousse dans des endroits qui seraient trop humides, même pour des plantes aussi peu sensibles à l'humidité que l'agrostide stolonifère. 
AVOINE JAUNÂTRE. (Trisetum flavescens, (L.) Beauv.)

Planche 6; graine, planche 26, fig. II .

\author{
Noms anglais: Yellow Oat Grass, Yellow False Oats, Golden \\ Oat Grass.
}

Description botanique: L'avoine jaunâtre est une plante vivace à souche courte, formant des touffes lâches. Les tiges, de un à deux pieds de hauteur, sont très grêles et feuillées, jusqu'au milieu environ. Des tiges secondaires naissent à la base des tiges principales. Les bourgeons dont elles naissent sont dans la gaine des vieilles feuilles, qui les recouvrent au début. Dans le développement de ces rejetons, la vieille gaine se fend, et la base des tiges principales est entourée des restes déchirés de la vieille gaine brune. Les tiges secondaires produisent un grand nombre de feuilles. Celles-ci, de même que celles de la tige principale, sont de texture très douce et couvertes de poils mous. Règle générale, leurs gaines sont également velues. Les fleurs sont arrangées en une panicule pyramidale, à rameaux étalés pendant la floraison. Après la floraison les branches se redressent et la panicule devient ainsi resserrée et étroite. Les nombreux épillets, d'abord verts, prennent à la floraison une belle teinte jaune d'or, d'où vient le nom de cette herbe. Après la floraison, leur couleur devient d'un brun jaunâtre terne. Les épillets renferment trois fleurs et chacune d'elles est enveloppée de deux glumes. Au dos de la glume extérieure se trouve une barbe délicate parfois courbée et tordue. La fécondation se fait de la même manière que chez le fromental.

Distribution géographique: L'avoine jaunâtre est propre à l'Europe, à l'Afrique du nord et aux parties tempérées de l'Asie; elle a été introduite dans le nord de l'Amérique. On ne l'a trouvée qu'une fois à l'état sauvage au Canada.

Habitat: Elle pousse naturellement dans les prairies un peu sèches, le long des chemins, sur les pentes et même sur les sommets des montagnes et dans les vallées.

Culture: L'avoine jaunâtre ne se montre pas difficile sur le choix du sol; il suffit que les autres conditions soient favorables. Elle 


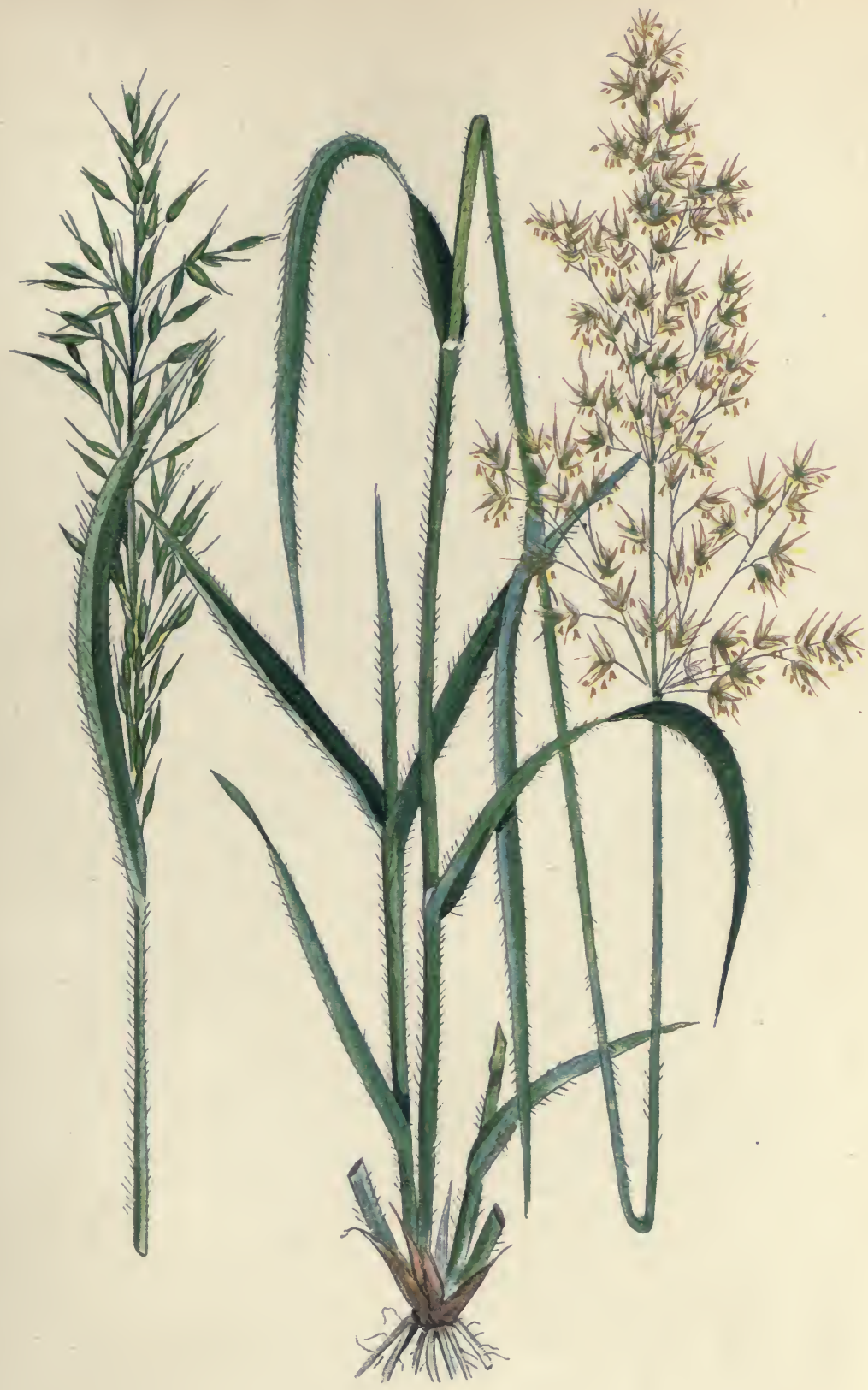

Planche 6 

supporte assez bien la sécheresse mais elle pousse mal sur un sol trop sec; il lui faut une terre assez humide, riche en matière organique et en chaux. Elle souffre plus d'une humidité excessive que de la sécheresse; l'eau stagnante exerce toujours un mauvais effet.

Végétation: L'avoine jaunâtre est moyennement hâtive; elle fleurit un peu plus tard que le dactyle pelotonné. Sur un sol et sous un climat convenables elle fournit une bonne levée en peu de temps et se propage rapidement.

Valeur agricole: Comme elle pousse en touffes on devrait toujours la semer avec d'autres herbes. Sa valeur principale dans les mélanges de foin est comme herbe de pied car elle augmente à la fois le rendement et la valeur nutritive. Après avoir été coupée elle produit un grand nombre de tiges et de feuilles nouvelles et le regain peut être utilisé avantageusement pour le foin ou le pâturage. Elle est recherchée par tous les bestiaux et dans certaines parties de l'Europe on la considère comme une des herbes fourragères les plus précieuses. $\mathrm{Au}$ Canada cependant les essais n'ont pas donné de brillants résultats. Semée seule, on doit mettre de vingt à vingt-cinq livres de graine à l'acre.

Graine: La graine de commerce de l'avoine jaunâtre est généralement très impure car on la récol te toujours en mélange avec d'autres plantes, surtout le fromental et le dactyle pelotonné et on sépare ensuite les graines au tamis. Elle est d'un brun-jaunâtre et pèse de cinq à six livres au boisseau.

Jamais la terre n'en imposa par de trompeuses apparences; elle dit franchement ce qu'elle peut ou ne peut point. Comme elle (la terre) n'exige que des connaissances simples et faciles, elle nous apprend à bien distinguer les gens lâches d'avec les gens actifs; elle paie avec usure les soins qu'on lui donne. Aussi, bien différente des autres arts, qui permettent aux paresseux de prétexter leur ignorance, elle fait hautement le procès à l'homme abject et vil; personne en effet ne se persuade qu'on puisse vivre sans le nécessaire; celui qui refuse de cultiver la terre, lorsqu'il n'a pas d'autre profession pour subsister, prouve donc qu'il projette de vivre voleur, brigand ou mendiant ou qu'il a tout à fait perdu l'esprit.-Xénophon, l'Economique, 434-355 a. J. C. 


\section{AVOINE ÉLEVÉE OU FROMENTAL. (Arrhenatherum elatius (L.) Beauv.)}

Planche 7; graine, planche 26, fig. 12.

Autres noms français: faux froment, faux seigle, avenat, fenasse pain-vin, ray-grass de France, arrhénantère.

Noms anglais: Oat Grass, False Oat Grass, Meadow Oat Grass, Tall Meadow Oat Grass, Tall Oat Grass.

Le fromental est une plante vivace, à souche courte, d'où naissent des tiges feuillées et des tiges stériles. Parfois la souche est enflée et les rejetons épais comme des bulbes à la base. Comme la souche est très courte les tiges sont serrées l'une contre l'autre et la plante pousse en touffes, mais ces touffes sont assez lâches. Les tiges ont de deux à cinq pieds de hauteur, parfois genouillées à la base et généralement vert foncé. Elles sont feuillées jusqu'au dessus du milieu. Les feuilles sont longues et larges, de texture assez molle, et généralement vert clair, parfois jaunâtre. Mélangé avec d'autres herbes, le fromental se reconnaît par cette couleur spéciale. Les fleurs sont portées en une panicule étalée qui ressemble assez à celle de l'avoine, d'où le nom d'avoine élevée. D'aspect général les épillets ressemblent assez à ceux de l'avoine. Chaque épillet contient deux fleurs, très dissemblables. La fleur inférieure, qui n'a que des étamines, est stérile et sa glume porte une longue barbe courbée; la glume de la fleur supérieure n'a généralement pas de barbe, les étamines et le pistil sont développés et la fleur est fertile. Chaque épillet ne produit donc qu'un seul grain. Parfois, mais rarement, les deux fleurs ont des barbes, et quelquefois une troisième fleur se développe au-dessus des deux fleurs normales. Les étamines et le pistil sont prêts à être fécondés en même temps. La fleur peut donc se féconder d'elle-même, mais il est probable que la fécondation croisée est très fréquente.

Distribution géographique: Le fromental est propre à la plus grande partie de l'Europe, au nord de l'Afrique et l'ouest de l'Asie. Il a été introduit d'Europe en Amérique du nord.

Habitat: Il pousse dans les prairies, sur les côtes, dans les champs ouverts, dans les clairières, le long des rives de la mer et sur les montagnes. 


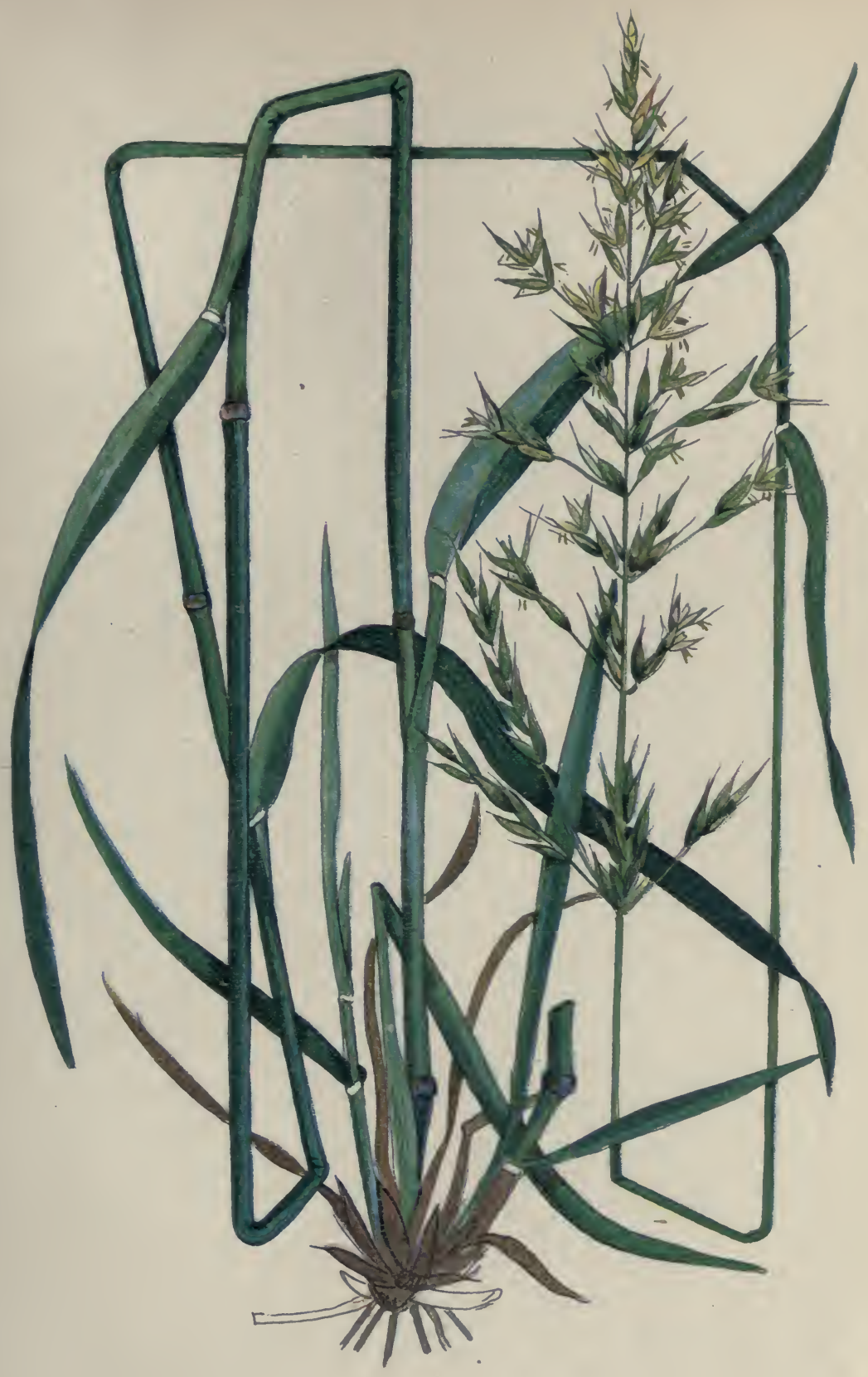

Planche 7

FROMENTAL

(Arrhenatherum elatius (L.) Beaul. ) 

Culture: Le fromental vient bien sur tous les sols assez frais. Cependant il pousse mieux sur les terrains élevés, secs et graveleux que sur les sols très humides. C'est sur les terres franches, légères et fraîches et sur les argiles pas trop lourdes ni trop humides qu'il rend le plus. Ses racines s'enfoncent profondément dans la terre, aussi supporte-il bien la sécheresse. Il est assez résistant à l'hiver.

Végétation: Il est assez facile d'obtenir un bon gazon de fromental, les jeunes plantes ont une végétation rapide et vigoureuse et produisent parfois des fleurs la première année. Si les autres herbes ne lui font pas une concurrence trop vive, il reste productif pendant bien des années. Le fromental commence à pousser de bonne heure au printemps et exige, pour son développement, à peu près le même temps que le dactyle pelotonné.

Valeur agricole: Pour le foin le fromental fait très bien en mélange avec le dactyle pelotonné, la fétuque des prés et le trèfle rouge. Comme il pousse en touffes, il ne devrait pas être semé seul mais toujours avec d'autres herbes. Pour le foin, on devrait le couper quand il est en fleurs. Si on attend, ne serait-ce que quelques jours après que les fleurs se sont ouvertes, il perd beaucoup de sa valeur nutritive car les tiges deviennent dures et ligneuses et perdent promptement les éléments nutritifs qu'elles renferment.

Pâturage: Le fromental se remet rapidement à pousser après avoir été coupé et résiste bien au pâturage. Cependant, il ne convient pas aussi bien pour le pâturage que pour la production du foin car la plante verte a un goût assez amer qui en éloigne les bestiaux tant que ceux-ci n'y sont pas habitués. On ne devrait donc le mettre qu'en petite quantité dans les pâtures et avec d'autres plantes fourragères. Quand on le sème seul, pour prairie ou herbage, on devrait mettre trente à trente-cinq livres de graine à l'acre.

Graine: Cultivé pour la production de la graine, le fromental devrait être coupé dès que les épillets commencent à prendre une teinte jaunâtre. De même que la folle avoine, il laisse tomber ses graines très tôt, c'est pourquoi il devrait être coupé de bonne heure. On récolte la graine et on la bat comme celle de l'avoine.

Qualité de la semence: La bonne semence de commerce est jaune verdâtre avec une teinte rougeâtre ou brunâtre. Elle pèse environ dix livres au boisseau. 


\title{
DACTYLE PELOTONNÉ (Dactylis glomerata L.)
}

\author{
Planche 8; graine, planche 26, fig. I3.
}

Autres noms français; Dactyle aggloméré, gramen pelotonné.

Noms anglais: Cocksfoot, Orchard Grass.

Description botanique: Le dactyle pelotonné est une plante vivace, à souche très courte. Les tiges, qui ont de deux à trois pieds de hauteur, sont serrées et entourées à la base par de nombreux rejetons feuillés. Les feuilles sont longues, larges, plates, de texture assez molle, et souvent pendantes, surtout par une journée chaude et sèche. On reconnait facilement le dactyle pelotonné, même dans la toute première phase de sa végétation par les rejetons de la base qui sont plats et à carène double. La forme particulière des rejetons provient du fait que les feuilles, dans le bourgeon, sont pliées ensemble le long de la ligne médiane. Les fleurs sont disposées en une panicule courte qui n'a généralement que deux ou trois branches principales,fortes et assez courtes. Quand elles sont en fleurs ces branches s'étalent comme les orteils d'une patte d'oiseau, d'où vient le nom anglais de "Cocksfoot." Pendant la période de maturation, elles se redressent graduellement, à la façon du bras qu'on lèverait au-dessus de la tête, et forment une panicule assez étroite. Les branches de cette panicule sont nues à la base, les épillets sont portés au sommet en groupes épais, d'un côté de la tige. Les épillets sont comprimés, l'un d'eux est légèrement creux, l'autre arrondi. Ils contiennent de deux à cinq fleurs dont chacune est entourée de deux glumes à pointes aiguës et fortement carénées. Les étamines se développent un peu plus tard que le pistil. La fécondation croisée est donc possible entre les fleurs de plantes différentes. Toutefois les fleurs d'une panicule sont très serrées, et il est tout probable que la plupart d'entre elles se fécondent elles-mêmes.

Distribution géographique: Le dactyle pelotonné est propre à l'Europe, à la zône tempérée de l'Asie et au nord de l'Afrique. Il a été introduit très tôt dans l'Amérique du nord. Quand il a été cultivé pour la première fois comme plante-fourragère en Angleterre, il y a environ cent cinquante ans, la graine avait été importée de Virginie. On le trouve maintenant dans toutes les régions tempérées de l'univers.

Habitat: Le dactyle pelotonné croît spontanément dans les prairies. les endroits incultes, le long des chemins, etc. Il se trouve 

dans les bois aussi bien que dans les clairières et il pousse mieux à l'ombre que les autres herbes de prairie. Il se rencontre souvent dans les vergers, d'où vient son nom anglais d'Orchard Grass. (Herbe de verger).

Valeur agricole: Le dactyle pelotonné est sans doute une des meilleures herbes fourragères, très estimée des cultivateurs. Il pousse remarquablement bien dans presque toutes les sortes de sols pourvu qu'ils ne soient pas trop humides. Il résiste très bien à la sécheresse.

Il est assez lent à s'établir; la première année les plantes sont petites et chétives. Elles se composent largement de rejetons feuillés naissant de la souche courte. La deuxième année les rejetens se montrent en grand nombre, au milieu d'eux paraissent des tiges florifères, mais ce n'est que la troisième année que l'on s'aperçoit de la productivité remarquable du dactyle. S'il est lent à atteindre son plein développement, d'autre part, une fois établi, il donne de forts rendements pendant bien des années. C'est une herbe précoce, prête à être coupée avant le mil. Pour cette raison il convient mieux que ce dernier en mélange avec le trèfle rouge.

Le dactyle pelotonné n'a pas d'égal en valeur nutritive pourvu qu'il soit coupé à l'époque voulue. C'est quand il est en fleurs, ou même un peu plus tôt, qu'il est le plus nourrissant et qu'il produit le plus. Il redevient ligneux a près la floraison et perd sa valeur nutritive. Il reprend rapidement après la coupe; les nombreux rejetons feuilles fournissent un excellent pâturage pour les chevaux et les bêtes à cornes. Toutefois il ne faut pas laisser le regain pousser trop haut, car il perd sa succulence en vieillissant.

Il y a peu de danger à la faire paître de trop près, à moins que la saison ne soit extrêmement sèche. Au contraire, la paissance au ras du sol empêche les plantes de devenir grossières et coriaces.

A cause de sa souche courte le dactyle pelotonné pousse en touffes serrées, pourvu qu'il ait assez de place et de nourriture. . Mais ce n'est pas là une caractéristique désirable, et il faut s'attacher à la corriger, soit en faisant des semis relativement épais, soit en le semant avec d'autres plantes fourragères. Dans chaque cas la formation en touffes sera moins marquée et l'on obtiendrait une herbe de texture plus fine et de qualité supérieure. Quand on le sème avec d'autres plantes fourragères, on ne doit choisir que ces variétés qui arrivent à maturité en même temps, telles que le trèfle rouge hâtif, le fromental et la fétuque des prés. Semé seul pour l'établissement de prairies ou de pâturages on ne doit mettre que vingt-huit à trente livres à l'acre; un peu moins pour la production de la graine. 
Production de la graine: Quand on le cultive pour la graine le même champ peut fournir cinq ou six récoltes de suite en autant d'années; c'est la troisième et la quatrième saison que l'on obtient les rendements les plus élevés. On augmente beaucoup la productivité en appliquant une couche de fumier sur le champ tous les ans. Le dactyle pelotonné cultivé pour la graine est prêt à être coupé trois ou quatre semaines après que les fleurs se sont formées. On détermine l'époque convenable à la coupe en battant quelques épis dans le creux de la main. S'il s'en détache une petite quantité de graine, c'est que la récolte est prête à être coupée. Si l'on coupe trop tôt, la graine est de qualité inférieure. On peut récolter avec une moissonneuse à grain ordinaire. On fait des gerbes plutôt petites que l'on rassemble par groupe de trois ou cinq en petites moyettes. On les laisse faner de deux à six semaines suivant l'état de la température puis on les bat sans les mettre en meules.

Qualité de la graine: La bonne graine est de couleur paille claire et ne contient qu'une petite quantité de graine nue ou d'épillets entiers, ou de groupes de graine qui ne sont pas séparés les uns des autres au battage. Elle garde assez bien sa vitalité pendant deux ans. Passé cet âge, elle perd une bonne partie de sa faculté germinative et on doit l'éviter.

\section{GRÉTELLE (Cynosurus cristatus L.)}

Graine, planche 26, fig. I4.

Nom anglais: Dog's Tail Grass, Crested Dog's Tail.

Description botanique: La crételle est une plante vivace à souche courte, qui pousse en touffes lâches, composées principalement de rejetons feuillus. Les tiges sont très grêles, assez rares, elles ont de un à deux pieds de hauteur. Les feuilles sont étroites, celles des tiges sont plus courtes que celles des rejetons de la base. Elles sont pliées dans le bourgeon et généralement légèrement concaves une fois entièrement développées. Les fleurs sont disposées en une panicule de forme et de construction particulières. Les rameaux sont très courts et tournés du même côté, ce qui donne à la panicule l'apparence d'un épi unilatéral. Chaque rameau porte deux épillets extrêmement dissemblables. L'un d'eux est composé de trois ou quatre fleurs normales, à étamines et pistils, et par conséquent fertiles; l'autre 
se compose d'un certain nombre d'écailles pointues, disposées en deux rangées comme les dents d'un peigne à deux faces. Cet épillet n'a ni pistil ni étamines, il est donc stérile. Quand la panicule est jeune les épillets fertiles sont cachés par les stériles, l'inflorescence ressemble à une crête. C'est cet aspect et cette forme de la panicule qui ont fait donner à la plante le nom de crételle.

Distribution géographique: La crételle est propre à presque toutes les parties de l'Europe et au sud-ouest de l'Asie. Elle a été introduite dans l'Amérique du nord, mais elle est assez rare au Canada.

Habitat: Elle croît spontanément dans les prairies, sur les côtes et les pentes des montagnes, le long des rives de la mer et sur les bords des chemins, etc.

Culture: Il lui faut un sol de qualité moyenne; les terrains bas et assez humides lui conviennent tout particulièrement. Elle ne pousse pas bien dans les localités extrêmement sèches, quoique ses racines assez profondes lui permettent de supporter la sécheresse.

Végétation: Elle atteint son plein développement la deuxième et la trcisième année après les semis. Elle se met à pousser assez tard et ce n'est que la deuxième année que l'on en obtient le plus fort rendement.

Valeur agricole: La végétation basse et assez touffue de cette plante permet de l'utiliser comme herbe de pied avec d'autres herbes. Elle est surtout utile dans les pâturages. C'est à elle que l'on attribue les excellentes qualités nutritives des pâturages d'Angleterre, de Hollande et de Schleswig-Holstein. On s'en sert généralement dans les mélanges pour les pelouses.

Graine: On obtient généralement la graine de crételle sur les plantes sauvages; elle est jaune orange à rougeâtre ou gris brun. Dans les échantillons de commerce les graines de différentes couleurs sont mélangées ensemble, ce qui donne à la masse une nuance brunjaunâtre. Le poids varie de trente à trente-deux livres au boisseau.

Du temps de l'ancienne Rome on semait, pour suppléer à l'insuffisance des prairies, du seigle qui se fauchait en vert, des lupins ou un mélange de pois, de lentilles, de fèves et de vesces, comme cela se pratique encore en quelques cantons, sous le nom de dragée; c'était le farrago des Romains.Marivault, Précis de l'histoire générale de l'Agriculture. 


\section{PÂTURIN COMPRIMÉ OU PÂTURIN BLEU DU GANADA (Poa compressa L.)}

Planche 9; graine, planche 26, fig. I5.

Noms anglais: Canada Blue Grass, English Blue Grass, Wire Grass, Creeping Poa, Smaller Blue Grass, Virginia Blue Grass, Canadian Blue Grass.

Description botanique: Le pâturin bleu du Canada est vivace. La souche souterraine est très traçante et émet de nombreuses branches dans toutes les directions. Une plante qui a pu se développer plusieurs années sans être dérangée forme généralement une plaque circulaire. La partie aérienne de cette plaque se compose de tiges éparpillées et de rejetons feuillés, ce qui donne un gazon épais, plus semblable à un tapis continu qu'à une touffe ouverte. Les tiges ont de un à deux pieds de hauteur, elles sont souvent genouillées à la base. Elles sont raides, à feuilles rares et fortement aplaties. Aucune autre espèce cultivée du genre Poa n'ayant de tiges aplaties, le pâturin bleu du Canada se reconnait à cette particularité. Les feuilles ont de un à trois pouces de longueur; elles ne sont pas aussi larges ni aussi nombreuses que celles du pâturin du Kentucky; elles sont d'un vert bleuâtre, parfois très glauque. Les fleurs sont en panicules, différentes de celles du pâturin du Kentucky. Dans cette dernière espèce, la panicule a généralement l'aspect d'une large pyramide, les branches inférieures sont nombreuses à chaque noeud. Quand elle est en fleurs, la panicule đu pâturin du Canada est généralement oblongue ou étroitement ovoïde; les rameaux sont courts et il n'y en a qu'un ou deux par noeud. Après la floraison la panicule devient étroite et resserrée, à branches droites. Les épillets sont comme ceux du pâturin du Kentucky et la fécondation se fait de la même manière.

Distribution géographique: Le pâturin bleu du Canada est propre à tous les pays eu:opéens et au sud-ouest de l'Asie. Il a été introduit dans l'Amérique du nord et a été trouvé au Canada il y a plus de cent ans. Il est maintenant l'objet d'une culture considérable dans le sud et le centre de l'Ontario.

Habitat: Il croît spontanément dans les endroits secs et ensoleillés, le long des chemins, sur les collines rocheuses et sur les rocs, et à partir du niveau de la mer jusque sur les hautes montagnes. On 


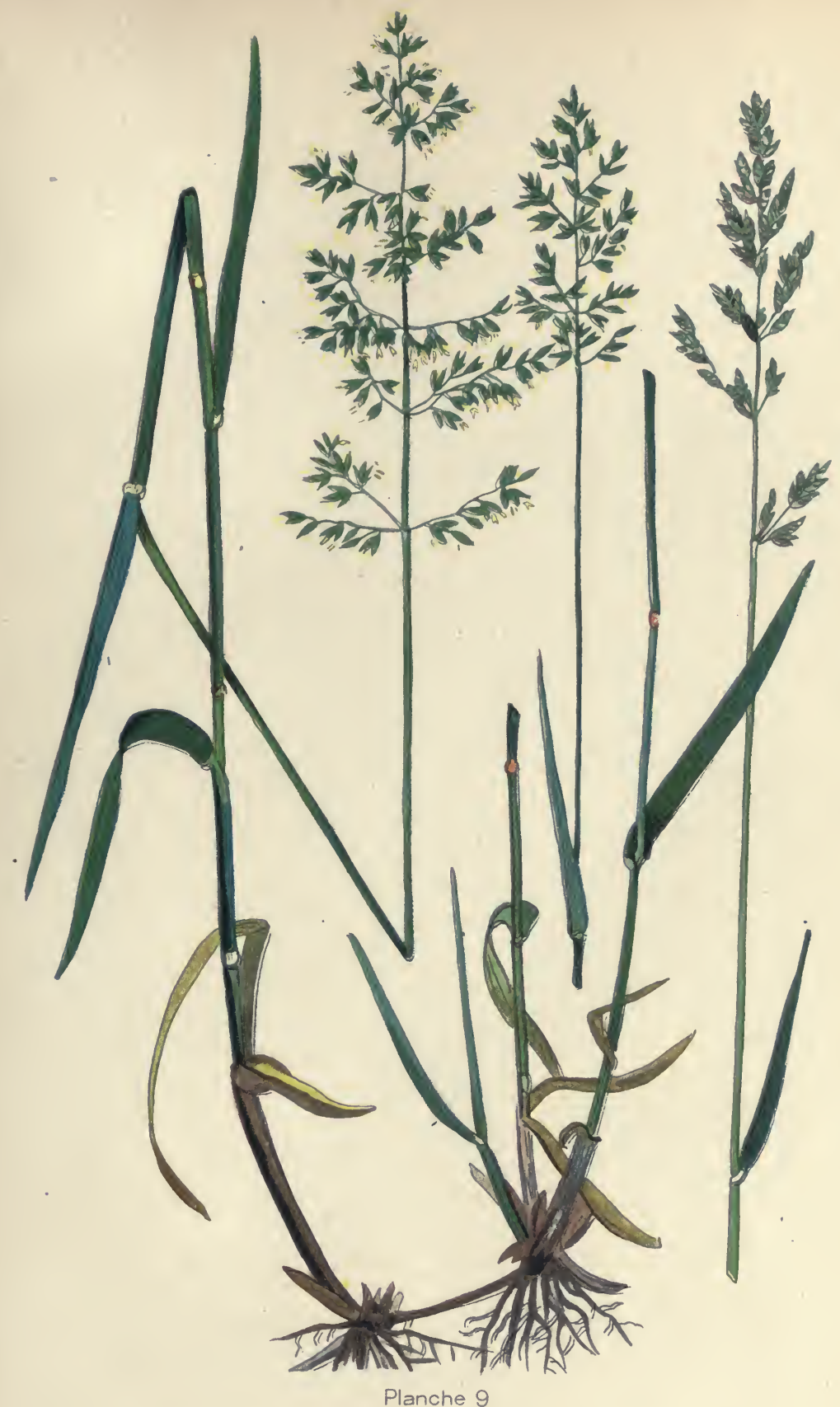

PÂTURIN COMPRIMÉ OU DU CANADA

(Poa compressa L.) 

le rencontre souvent dans les sols pauvres et graveleux où d'autres plantes ont de la peine à s'établir.

Culture: Au Canada on choisit généralement pour le pâturin les terres dures, pauvres, argileuses ou argilo-sableuses, parce qu'il y pousse tandis que les autres plantes y produisent peu de chose.

Climat: Il est peu difficile au sujet du climat. La sécheresse extrême, qui serait désastreuse à la plupart des autres plantes fourragères, arrête sa végétation, mais celle-ci reprend à la première pluie et de nouvelles tiges et feuilles naissent de la souche. Il résiste aux gelées et aux dégels consécutifs sans souffrir. C'est pourquoi l'on trouve si souvent des parcelles de pâturin dans les parties basses des luzernières mal drainées, dans certaines localités du sud de l'Ontario.

Végétation: Par son mode de propagation et son aptitude à supporter sans souffrir les conditions désavantageuses, le pâturin du Canada ressemble beaucoup à certaines mauvaises herbes et particulièrement au chiendent. Dans un sol riche où viennent avec succès d'autres plantes fourragères telles que la luzerne, le pâturin du Canada n'est en somme qu'une mauvaise herbe, difficile à extirper, et que bien des cultivateurs regardent comme un fléau.

Fourrage: On s'en sert peu pour faire du foin à cause de sa faible productivité mais on prétend que le foin en est sain et nourrissant pour les chevaux.

Pâturage: Il est surtout utile comme herbe permanente dans les pâturages. Il ne faut pas le laisser trop longtemps car il perd sa succulence à la longue. On peut sans danger le faire brouter de très près; la paissance à ras provoque la végétation et le rend plus attrayant pour le bétail. Comme herbe de pâturage il est riche et nourrissant, surtout pour la production de bœuf ou de mouton. Il est également avantageux dans la production du lait. On le recommande pour les pelouses, dans les sols argileux qui manquent de chaux et il forme généralement partie des mélanges de commerce pour les pelouses. Vingt à trente livres de graine à l'acre suffisent.

Production de la graine: Dans la production de la graine on obtient généralement les plus forts rendements de nouveaux champs ou de récoltes volontaires qui viennent après le blé ou d'autres 
grains. On doit faucher quand les panicules sont d'un jaune foncé. Le fanage et le battage n'offrent aucune difficulté; ils se font de la même manière que pour le mil. On peut se servir de la batteuse à grain ou à trèfle. Cette dernière est généralement préférée.

Qualité de la graine: En masse la graine est d'un brun jaunâtre, parfois terne, un peu plus foncée que celle du pâturin bleu du Kentucky; sous tous les autres rapports les graines de ces deux plantes sont très semblables dans le commerce. Généralement les graines du pâturin du Canada sont plus tronquées, avec une extrémité plus large, et les nervures latérales des glumes sont absentes ou indistinctes.

Le poids légal d'un boisseau de graine est de quatorze livres.

\section{PÂTURIN DES PRÉS OU PÂTURIN BLEU DU KENTUGKY (Poa pratensis L.)}

Planche I0; graine, planche 26, fig. I7.

Noms anglais: Blue grass, June grass, Spear grass, English grass, Green grass, Bird grass, Smooth stalked Meadow grass, Common Meadow grass, Kentucky blue grass.

Description botanique: Le pâturin bleu du Kentucky ou pâturin des prés est une plante vivace, à souche rampante, émettant des stolons et des tiges feuillées. Ces stolons sont des tiges souterraines qui portent des écailles sans couleur au lieu de feuilles vertes. Ils rampent à la surface du sol, émettant des racines aux jointures, et produisent finalement, à leur extrémité, des tiges droites et feuillées. Les rejetons feuillés sont droits dès le début de leur végétation et se lèvent en touffes de la base même des tiges. Ils sont ronds et n'ont d'abord que des feuilles mais se développent plus tard en tiges florifères. Les tiges ont de 6 pouces à 3 pieds de hauteur, elles sont parfaitement lisses. Les feuilles des tiges sont relativement courtes, d'un pouce à deux de longueur seulement, leur sommet est contracté, un peu à la façon du bout d'un canot. Les feuilles des rejetons de la base sont plus longues et généralement plus étroites que les feuilles de la tige. Ces feuilles accusent beaucoup de variation au point de vue de la couleur de la forme et de la grosseur mais elles ont une caractéristique qui leur est commune à toutes, c'est que la ligule est très 


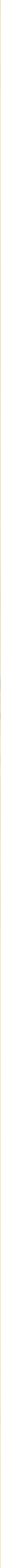

PÂTURIN DES PRÉS OU DU KENTUCKY

(Poa pratensis L.) 

courte et tronquée. Elles sont assemblées en une panicule qui est de forme pyramidale pendant la floraison; plus tard cette panicule devient plus ou moins resserrée. Chaque rameau de la panicule porte plusieurs épillets; ces épillets sont d'un vert bleuâtre,-d'où vient le nom de pâturin bleu-mais parfois ils ont une teinte pourpre ou violette. Règle générale, un épillet a quatre ou cinq fleurs et chacune de ces fleurs est enveloppée de deux glumes d'égale grosseur. L'arrangement des étamines et du pistil indique que la fécondation croisée s'accomplit facilement, mais il n'y a pas de doute que beaucoup de fleurs se fécondent d'elles-mêmes.

Distribution géographique: Le pâturin du Kentucky est une plante cosmopolite, répandue dans tout l'univers en dehors des tropiques. Il est propre à l'Europe, à l'Afrique du nord, à la Sibérie et à l'Amérique du nord. Il est également propre à l'Australie et aux parties méridionales de l'Amérique du sud.

Habitat: Il croît spontanément dans presque toutes les localités et il résiste aux conditions les plus rigoureuses. Son endroit de prédilection est la prairie mais il est commun dans d'autres endroits. Par exemple il pousse le long des chemins, sur les bords des bois, sur les collines sèches, dans les marécages, le long des rives de la mer ou bien au pied des glaciers du Groenland et sur les sommets des montagnes du Sahara. On le cultive beaucoup dans tous les pays où l'agriculture est une industrie importante.

Culture: Le pâturin du Kentucky ne se développe bien que dans les terres de bonne qualité. Les extrêmes nuisent à sa végétation, les sols pauvres, sableux ou argileux lui conviennent aussi peu que les lourdes argiles. Il atteint son développement maximum sur les fonds bien meubles et riches en humus, surtout si la terre contient assez de chaux. Dans les régions à chaux du Kentucky et du Tennessee, on considère que le pâturin bleu est la reine de toutes les herbes de pacage et l'on dit dans quelques-uns des Etats américains que tous ceux qui ont des terres à chaux ont également le pâturin.

Climat: Il préfère un sol assez frais mais il supporte bien la sécheresse. Il est extrêmement rustique; les gelées et les couches épaisses de neige ne lui font aucun tort.

Végétation: Le pâturin du Kentucky s'établit assez lentement. La première année il ne produit aucune tige, seulement quelques 
rejetons feuillés qui apparaissent en petites touffes éparpillées. L'année suivante, ces touffes sont moins clairsemées parce que les stolons souterrains ont développé de nouveaux rejetons feuillés qui occupent presque tout l'espace entre les branches de la végétation de la première année, et quelques tiges florifères se sont formées. A partir de la troisième année, si les conditions sont favorables, il y a un gazon épais, serré, qui couvre presque tout le sol. La végétation est très rapide au printemps et la plante fleurit à peu près en même temps que le dactyle pelotonné.

Valeur agricole: Dans les prés destinés à la production du foin, le pâturin du Kentucky devrait être coupé quand il est en fleurs; c'est à ce moment qu'il renferme le plus de nourriture. Après avoir été coupé il pousse assez lentement et comme le regain se compose principalement de feuilles, on ne peut compter sur une deuxième récolte. Dans les mélanges il fait une bonne herbe de pied et ajoute beaucoup à la masse du foin de la première coupe. C'est une des meilleures herbes pour les pelouses.

Pâturage: Il est très apprécié comme herbe de pacage; il pousse tôt au printemps, fournit une nourriture de qualité supérieure et est brouté avec avidité par toutes les sortes de bestiaux, dont il provoque l'engraissement. Semé seul pour foin ou pour pâturage, on doit mettre de vingt à trente livres de graine à l'acre.

Graine: Les graines de commerce du pâturin du Kentucky viennent presque toutes de quelques comtés du Kentucky, au coeur même de la région du pâturin. On les récolte à la main ou avec des machines qui dépouillent les plantes de leurs graines et les ramassent en même temps. Les graines sont prêtes à être récoltées quand les panicules sont jaunes; à ce moment la graine est assez mûre. Elle finit de mûrir après avoir été récoltée. Pour compléter cette maturation il faut brasser la graine fréquemment, trois fois par jour au moins durant les premiers jours, pour que l'air puisse avoir accès à toutes les parties de la masse et empêcher la fermentation. La graine qui n'est pas bien séchée prend une couleur d'un gris poussiéreux et contracte une odeur de moisi. Sa vitalité est très affaiblie sinon entièrement détruite.

Qualité de la graine: La bonne semence de commerce est brun jaunâtre. Les graines que l'on fait sortir des épillets portent à la base une touffe de longs poils semblables à une toile d'araignée. On ne 
trouve pas ces poils dans la graine du pâturin du Canada: on peut donc facilement distinguer cette dernière, quand elle est fraîche, de la graine du pâturin du Kentucky. Mais ces poils disparaissent au cours des frottements occasionnés par le fanage et les nettoyages, et alors la graine de commerce du pâturin du Kentucky et du pâturin du Canada se ressemblent beaucoup. Règle générale, la graine du premier est pointue, les nervures des glumes sont saillantes tandis que la graine du dernier est tronquée et les nervures des glumes sont peu apparentes.

Le poids légal d'un boisseau de graine de pâturin est de quatorze livres.

\section{PÂTURIN COMMUN OU PÂTURIN RUDE (Poa trivialis L.)}

Nom anglais: Rough stalked meadow grass.

Description botanique: Le pâturin commun est très semblable au pâturin du Kentucky. C'est une plante vivace à souche courte, d'où naissent des tiges et des rejetons feuillés. Ces derniers sont dressés ou rampants. Les rejetons dressés paraissent en grand nombre à la base des tiges, et les plantes sont en touffes plus visibles que le pâturin du Kentucky. Les stolons rampants naissent de la même manière mais s'étendent dans une direction horizontale. Ils correspondent ainsi, d'une certaine façon, aux stolons du pâturin du Kentucky mais les différences sont très sensibles. Les stolons du pâturin du Kentucky sont souterrains et écailleux, tandis que les stolons du pâturin commun rampent toujours à la surface et portent des feuilles vertes, de grosseur normale. Ils émettent des racines aux jointures et leurs extrémités finissent par se redresser et produire des rejetons normaux feuillés, dont le développement est très semblable à celui des rejetons correspondants du pâturin du Kentucky. Tous les jeunes rejetons du pâturin commun sont nettement aplatis tandis que ceux du pâturin du Kentucky sont ronds. Les tiges, de un à trois pieds de hauteur, sont feuillées jusqu'au-dessus du milieu; elles sont rudes immédiatement au-dessous de la panicule, d'où le nom significatif de pâturin rude. Les feuilles sont nombreuses, généralement d'un vert foncé, à gaine grossière et à ligule longue et aiguë. La panicule est semblable à celle du pâturin du Kentucky, mais elle est généralement plus étalée et vert foncé. 
Distribution géographique: Le pâturin commun est propre aux régions tempérées de l'ancien monde; il a été introduit dans l'Amérique du nord.

Habitat: Il croît spontanément dans les prairies riches et fraîches, au bord des bois, dans les taillis, les jardins, les champs cultivés, le long des chemins et des fossés, à partir du niveau de la mer jusque sur les hautes montagnes.

Culture: Le pâturin commun préfère les terrains bas où le sol est riche et l'humidité abondante.

Climat: Il ne résiste pas à la sécheresse aussi bien que le pâturin du Kentucky. Ceci provient de ce que les stolons rampants sont au-dessus de la terre et plus exposés que ceux du pâturin du Kentucky qui sont souterrains. Il reprend rapidement après une sécheresse et peut être cultivé sur les terres irriguées pourvu que les autres conditions soient favorables.

Végétation: Il prend racine beaucoup plus promptement que le pâturin du Kentucky. La première année les plantes produisent des stolons rampants qui s'enracinent aux juintures et forment des branches dressées. La croissance est rapide et le sol est bientôt recouvert. La deuxième année, les plantes ont atteint leur plein développement et donnent le rendement le plus éleve.

Valeur agricole: C'est quand il est en fleurs quẻ le pâturin commun donne le fourrage le plus nourrissan., c'est dunc à ce moment qu'on doit le couper pour en faire du foin. Toutefois, sur les terres humides, il est bon de le couper plus tôt car les tiges pourrissent aisément à la base. C'est une plante précieuse dans les mélanges que l'on fauche une fois par an, mais quand on désire plus d'une récolte on fera bien de le remplacer par une autre plante car son regain est insignifiant. Il est précieux comme herbe de pacage à cause de sa végétation rapide et vigoureuse et $\mathrm{du}$ fait qu'il résiste très bien au piétinement.

Graine: Dans le centre de l'Europe on se procure généralement la graine du pâturin commun sur des plantes sauvages, dont on la dépouille à la main. Au Danemark la graine est cultivée pour le commerce sur une assez grande échelle. On sécolte du même champ de dẹx à quatre ans de suite. On sème en zénéral de quinze à vingt livres de graine à l'acre. 
Qualité de la graine: Les graines sont munies de poils en forme de toile d'araignée, semblables à ceux du pâturin du Kentucky et les graines des deux plantes sont semblables sous tous les rapports. La seule différence c'est que, dans les graines du pâturin commun, les glumes ont des nervures plus saillantes. Comme la graine du pâturin du Kentucky est beaucoup moins coûteuse on la substitue souvent à celle du pâturin commun. En fait, il est assez difficile d'obtenir de la graine pure de ce dernier.

\section{PÂTURIN DES BOIS (Poa nemoralis L.)}

Nom anglais: Wood meadow grass.

Description botanique: Le pâturin des bois est vivace, à souche courte, et pousse en touffes lâches; ces touffes se composent principalement de tiges florifères stériles; plus tard dans la saison, généralement après que la graine est mûre, des stolons feuillés se développent. Les tiges sont plus grêles que dans les autres espèces de $P o a$ ici décrites, et les feuilles des tiges beaucoup plus longues. Ces feuilles sont très étroites, généralement aussi larges que la tige, et la ligule est extrêmement courte ou absente. La panicule est mince, d'oblongue à ovoïde pendant la floraison, et plus tard resserrée et étroite. Les épillets ne sont que d'une couleur, généralement vert ou brun clair. Ils contiennent de une à cinq fleurs.

Distribution géographique: Le pâturin des bois est indigène à l'Europe et aux régions tempérées de l'Asie; on prétend qu'il est également indigène au Canada. Ceci est douteux cependant, car les plantes canadiennes, généralement appelées Poa nemoralis, sont très différentes du vrai type européen. Ainsi on rencontre le Poa nemoralis dans les prés, le long des bois et même dans la prairie ouverte. Le vrai pâturin des bois se trouve dans les bois et ne vient pas bien dans les endroits exposés, du moins pas dans les prairies ouvertes. Il est très répandu dans les bois de hêtres où l'ombre est épaisse, ou sous les arbres décidus.

Valeur agricole: Le pâturin des bois qui appartient aux localités ombragées n'a évidemment pas de valeur en agriculture, soit pour le foin ou pour le pacage, sauf dans des parcs ombragés ou dans des bois ouverts, où l'on désire avoir du fourrage. Il est très utile pour établir des pelouses sous les arbres. 
Graine: La plupart de la graine du commerce vient de l'Allemagne où on la cueille sur des plantes sauvages pour la nettoyer à la main.

Qualité de la graine: La graine est très semblable à celle du pâturin du Kentucky et du pâturin commun mais elle en diffère par les nervures peu marquées de ses glumes; sous ce rapport elle ressemble beaucoup à celle du pâturin des marais dont elle diffère par ses glumes à bout pointu. Elle est brun jaunâtre vif, avec une teinte pourprée.

\section{PÂTURIN DES MARAIS (Poa palustris L.)}

Autres noms latins: Poa flava L., Poa serotina Ehrh., Poa triflora Gilib.

Noms anglais: Fowl meadow grass, False Red Top.

Description botanique: Le pâturin des marais est une plante vivace, à rhizomes courts, qui produisent de rares rejetons courts et feuillés. Il ressemble au pâturin commun, mais il forme des touffes plus ouvertes qui se composent principalement de tiges florifères. Ces tiges sont rarement très droites, elles sont ascendantes et leur base repose à plat sur le sol. A la base elles émettent des racines et des branches secondaires qui se développent en tiges feuillées ordinaires. Les tiges ont de deux à cinq pieds de hauteur, elles sont feuillées jusqu'au dessus du milieu. Les feuilles des tiges sont longues et étroites, à texture molle et à couleur vert clair. La ligule est généralement longue mais tronquée. Les panicules sont larges et portent aux jointures de nombreuses branches. A la floraison les branches sont très étalées; plus tard elles se redressent et forment une panicule étroite. Chaque épillet contient de trois à six fleurs d'une couleur singulière. La partie basse est verte, le sommet est jaune ou brun avec un lustre foncé ou bronzé. Les épillets ont ainsi deux couleurs et l'effet de la panicule entière est très différent de celui du pâturin du Kentucky ou du pâturin commun.

Distribution géographique: Le pâturin des marais est indigène à l'Europe, aux régions tempérées de l'Asie et à l'Amérique du nord.

Habitat: Il croît spontanément dans les prairies fraîches, les fossés et le long des rives de la mer et des cours d'eau, etc. 
Culture: Il atteint la perfection dans les prairies humides où le sol est riche en matière organique, et il pousse bien sur les terres argileuses ou argilo-sableuses qui sont inondées de temps à autre.

L'eau stagnante ne lui fait aucun mal. Il convient donc mieux que le pâturin commun dans les localités marécageuses, où ce dernier est exposé à pourrir à la base des tiges.

Végétation: Il est assez facile d'obtenir une levée de cette plante mais il lui faut deux ou trois ans pour arriver à complet développement. Dans des conditions favorables il supplante à la longue les autres herbes et forme un gazon épais et persistant. Il se met à pousser plus tard que le pâturin du Kentucky et fleurit généralement quelques semaines plus tard que ce dernier. Il reste vert et assez succulent longtemps après avoir fleuri.

Valeur agricole: Le pâturin des marais est généralement cultivé pour le foin et il rend beaucoup dans les localités basses bien pourvues d'eau. Il donne un fourrage riche, très apprécié de toutes les sortes de bétail. Son regain est bon; il a donc une valeur considérable comme plante fourragère. On le sème généralement avec d'autres herbes comme l'agrostide et le mil, (fléole des prés.)

Graine: On cultive peu le pâturin des marais pour sa graine; les cultures les plus importantes sont en Bohême et en Autriche.

Qualité de la graine: Les graines portent à la base, avant d'être nettoyées, une touffe de poils en forme de toile d'araignée; elles sont d'un brun jaunâtre, souvent avec une teinte rougeâtre ou pourpre. Elles diffèrent des graines du pâturin du Kentucky et du pâturin commun principalement. dans les glumes qui sont assez tronquées et ont des nervures indistinctes.

\section{LA GLYCÉRIE AQUATIQUE (Glyceria aquatica, (L.) Wahlb.)}

Autre nom français: Pâturin aquatique.

Nom anglais: Water meadow grass.

Graine, planche 26, fig. I7.

Description botanique: La glycérie aquatique est une plante vivace, à souche très longue, épaisse et rampante. Les tiges, généralement enracinées à la base, sont épaisses et atteignent jusqu'à neuf pieds de hauteur. Elles sont feuillées jusqu'au-dessus du milieu et ces feuilles sont longues et larges. La plante entière a une couleur vert clair singulier, ou vert jaunâtre. On reconnait aisément la 
glycérie aquatique par les deux taches jaune ou brun jaunâtre à l'extrémité supérieure de la gaine. Les fleurs sont portées en panicules étalées qui ont parfois un pied et demi de long sur une largeur presque égale; les branches sont très nombreuses et portent un grand nombre d'épillets. Ces épillets sont d'abord d'un vert jaunâtre, mais après avoir fleuri ils deviennent brun clair, parfois avec une nuance de pourpre. Un épillet contient de cinq à huit fleurs dont chacune est entourée de deux glumes.

Distribution géographique: La glycérie aquatique est propre à presque toute l'Europe et aux régions tempérées de l'Asie. Dans l'Amérique du nord une espèce étroitement alliée, Glyceria grandis Wats, qui s'en distingue principalement par ses fleurs plus petites, prend la place de la Glyceria aquatica et a peut-être une valeur égale en agriculture.

Habitat: La glycérie aquatique se rencontre sur les rives boueuses des lacs et des cours d'eau, où elle couvre parfois de vastes superficies à l'exclusion de toute autre herbe. Elle pousse superbement dans les eaux peu profondes, à fond marécageux, où les souches rampantes ne rencontrent aucune résistance. Dans les terrains secs, où les souches ne peuvent se développer convenablement, la végétation en souffre et l'herbe est de pauvre qualité.

Valeur agricole: Malgré son fort développement la glycérie aquatique est de texture assez molle et peut être broutée au ras du sol par les chevaux et les bovins. Les chaumes sont succulents, savoureux et très nutritifs, surtout quand ils sont jeunes, et très recherchés des bestiaux. Dans certains pays européens on connaissait déjà sa valeur au dix-huitième siècle et cependant elle est peu cultivée.

Graine: La graine est rare et souvent on ne trouve dans le commerce que les souches ou rhizomes. Les graines sont la:gement oblongues; la glume extérieure est tronquée et munie de sept nervures saillantes. La graine non dépouillée de sa balle est généralement verdâtre ou brun jaunâtre. La graine nue est brun noirâtre luisant, ovale à oblongue; elle a à peu près la longueur de la graine de luzerne. 


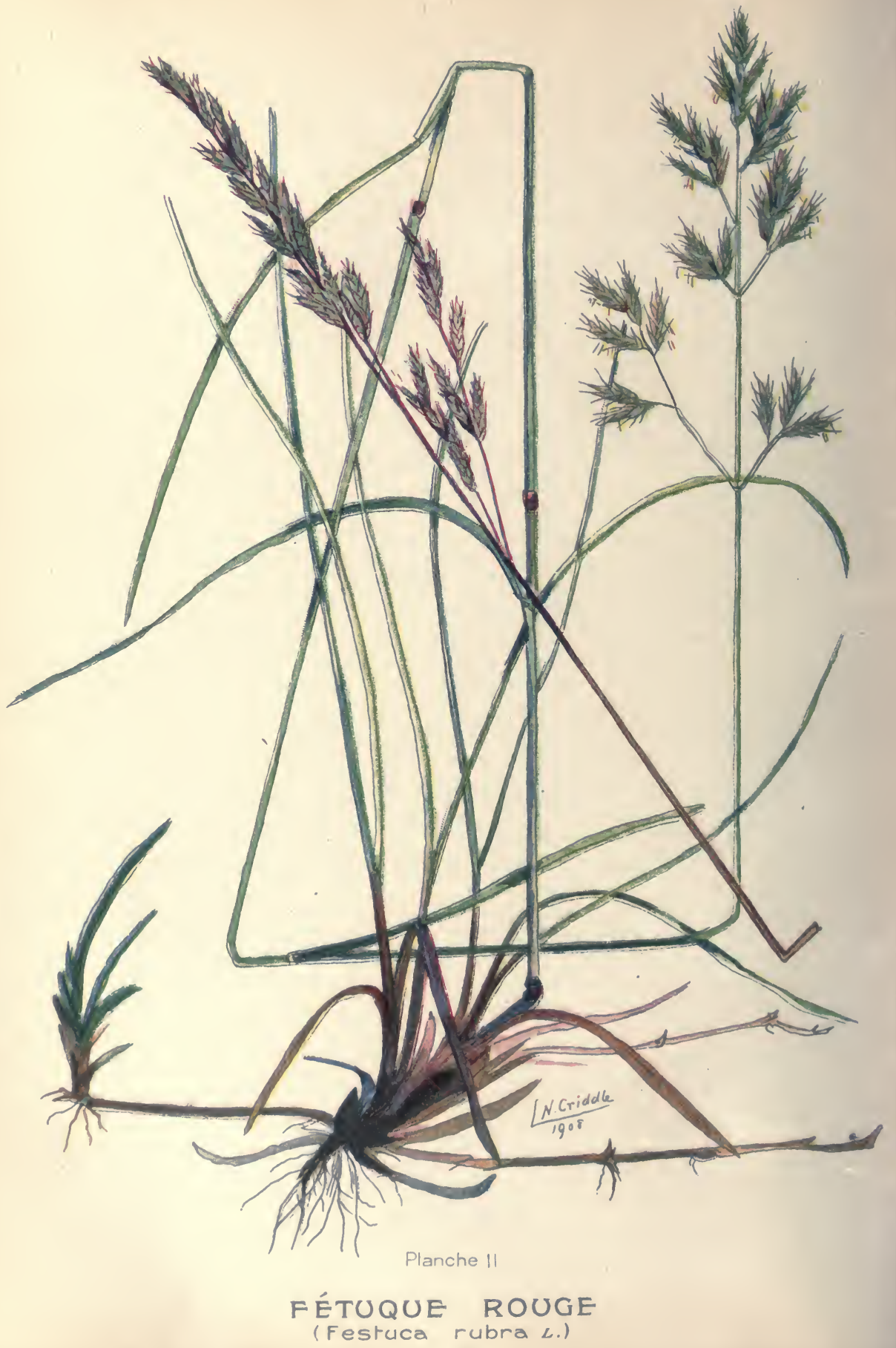




\section{FÉTUQUE ROUGE (Festuca rubra L.)}

Planche II ; graine, planche 26, fig, I8.

Noms anglais: Creeping Fescue, Red Fescue.

Description botanique: La fétuque rouge est une plante vivace, à souche longue, rampante et souterraine, aux nœuds de laquelle naissent les tiges et rejetons aériens. Pour cette raison il ne se forme pas de vraies touffes, mais des tapis plus ou moins étendus, à tiges éparpillées. Ces dernières ont de dix-huit à trente-six pouces de hauteur, elles sont lisses et rondes. Des tiges secondaires naissent des bourgeons, dans les gaines des feuilles de la base. Quand ils se développent, ces rejetons passent au travers de la gaine en la déchirant en fragments. Les restes d'écailles brunes et les fils que l'on trouve toujours à la base des tiges et des rejetons de la fétuque rouge représentent les restes des gaines. Ces restes permettent de distinguer la fétuque rouge facilement de la fétuque ovine. Les feuilles sont enroulées dans le bourgeon, de même que celles de la fétuque ovine, mais tandis que toutes les feuilles sont enroulées dans cette dernière, il n'y a que celles de la base qui se maintiennent en cet état dans la fétuque rouge; les feuilles de la tige sont plates. Les fleurs sont portées en panicule, comme celles de la fétuque ovine, mais, règle générale, la panicule est plus large et souvent un peu penchée. Les épillets, dont chacun renferme de quatre à six fleurs, sont de diverses couleurs, souvent d'un brun rougeâtre d'où le nom de fétuque rouge. La glume extérieure de la fleur a une barbe, généralement plus longue que dans la fétuque ovine.

Distribution géographique: Sa distribution géographique est à peu près la même que celle de la fétuque ovine.

Habitat: La fétuque rouge croît spontanément dans les prés et les pacages, le long des rives de la mer, sur les montagnes, dans les champs et les bois.

Culture: Elle est un peu plus difficile sur le choix du sol que la fétuque ovine, et ne vient pas bien dans les terres trop compactes ou extrêmement sèches où elle ne peut développer ses racines rampantes. Les sols ouverts, sableux, graveleux, contenant suffisamment d'humidité, lui conviennent mieux. Elle supporte assez bien la sécheresse, mais pas autant que la fétuque ovine, et elle résiste bien aux grands 
froids. Ses racines rampantes étant à la surface, elle peut se développer dans les sols peu profonds.

Végétation: La fétuque rouge ne commence pas à pousser aussi tôt au printemps que la fétuque ovine. C'est au moment de la floraison qu'elle contient le plus de nourriture, car les feuilles de la base sèchent ou deviennent coriaces et peu succulentes bientôt après. Elle reprend rapidement après avoir été fauchée ou broutée. De nombreuses feuilles nouvelles naissent des souches souterraines. Pour cette raison elle fait une assez bonne herbe de pied dans les mélanges à foin.

Valeur agricole: Malgré sa valeur nutritive assez faible, la fétuque rouge a des qualités qui en font une herbe précieuse pour les pacages et les pelouses. Elle supporte bien le piétinement et les fauchages et forme des tapis fermes et durables de gazon solide qui lient le sol sur les terres sableuses ou graveleuses. On cultive des variétés naines, d'une texture extra fine, et l'on en garde la graine pour les pelouses.

Graine: La graine de la fétuque rouge se récolte généralement sur les plantes sauvages. Elle est de couleur paille, souvent avec une teinte rouge ou violette; elle est généralement un peu plus longue que celle de la fétuque ovine. Elle pèse de dix à quinze livres au boisseau.

O trop heureux l'homme des champs, s'il savait quel est son bonheurl Loin du bruit des armes et des discordes, la terre, dans sa pleine justice, lui fournit d'elle-même et sur son propre fonds une abondante nourriture. Sans doute, ce n'est point auprès de lui que se dressent ces palais aux portes orgueilleuses...... mais ce qui ne lui manque pas en revanche, c'est le calme et l'absence de soucis, c'est une vle exempte de plaisirs trompeurs, et riche de ressources variées; ce sont les doux loisirs et les larges horizons; les grottes, les lacs d'eau vive, et les frais vallons, les mugissements des boeufs et le sommeil agréable à l'ombre des arbres; là sont les pâturages et les halliers où gitent les hôtes des bois, une jeunesse dure à la fatigue et contente de peu; là on pratique le culte des Dieux et le respect de la vieillesse; c'est là que la Justice, avant de quitter la terre, a laissé la trace de ses derniers pas.Virgile, Les Géorgiques, Traduction d'Henri Lantoine.

Aux anciens il fallait la plaine et la charrue,

Le grand air dort le souffle ondoie au front des blés;

Les nouveaux ont quitté ie sillon pour la rue,

Et, jeunes, des désirs malsains les ont troublés.

Leurs bras n'étaient pas faits pour les besognes viles,

Et le joug paternel pesait à leur fierté.

Les voyez-vous, épars sur les chemins des villes,

Tous ces riches d'espoir qu'attend la pauvreté.

Ils ont, ces émigrants, ambitie : $x$ ou lâches,

Gêné les citadins, gêné les artisans.

Dieu ies avait crés pour de plus nobles tâches,

Les paysans devalent rester des paysans.

PAUL HAREL, Voix de la Glèbe. 


\section{FÉTUQUE OVINE (Festuca ovina L.)}

Autres noms français: Coquiole, petit foin, fétuque des brebis.

$$
\text { Graine, planche 26, fig. } 19 .
$$

Description botanique: La fétuque ovine est une plante vivace, formant des touffes épaisses. Les tiges grêles et nombreuses sont plus ou moins anguleuses et ont de huit à vingt-quatre pouces de hauteur. Elles sont entourées à la base de nombreux rejetons qui naissent des bourgeons dans les gaines persistantes des vieilles feuilles de la racine. Ces rejetons apparaissent à l'ouverture de la gaine et non pas à la base de celle-ci, comme dans la fétuque rouge. Pour cette raison les gaines ne sont pas découpées en fragments comme dans la fétuque rouge, mais elles restent entières, sauf à la partie supérieure, et la base des tiges n'est pas entourée de fragments écaillés et de bandes. Les feuilles sont très étroites, généralement vert pâle; celles des rejetons de la base ont de trois à quatre pouces de long et celles de la tige n'ont qu'un pouce environ. Elles sont enroulées dans les bourgeons et persistent dans cet état même quand elles sont entièrement développées. C'est à cause de cela que les feuilles de la fétuque ovine ont souvent un aspect hérissé. Les fleurs sont portées en une panicule unilatérale de un à quatre pouces de long. Les rameaux de la panicule s'étalent pendant la floraison mais se redressent plus tard, en forme d'épi plus étroit. Les épillets sont verts, souvent avec une teinte violette. Chaque épillet renferme de trois à quatre fleurs et chaque fleur est entourée de deux glumes. La glume extérieure porte au sommet une barbe courte.

Distribution géographique: La fétuque ovine est propre à l'ancien monde; elle se rencontre de l'Angleterre au Japon et du Spitzberg et de l'Islande à l'Afrique du nord et l'Himalaya. Elle est également propre au Canada et à certaines parties des Etats-Unis. Toutefois, un bon nombre d'espèces ont été introduites d'Europe où elle est cultivée depuis environ 1820 .

Habitat: Elle croît spontanément dans toutes les localités sèches, dans les pacages secs, les champs sablonneux, sur les rocs, etc., à partir des rives de la mer jusqu'aux régions alpines. En Europe on la trouve à huit mille pieds d'altitude au-dessus du niveau de la mer.

Gulture: La fétuque ovine prospère sur les sols secs et stériles où la plupart des autres graminées ne peuvent s'établir ou périssent de sécheresse et de manque de nourriture. Elle endure presque $28550-9$ 
toutes les rigueurs de la nature sans en souffrir sérieusement et se relève promptement après de longues périodes de souffrance. Le manque d'humidité peut arrêter sa végétation, la forte sécheresse peut rendre ses feuilles si sèches qu'elles se cassent au moindre contact, mais que la plante, morte selon toute apparence, reçoive un peu d'eau, elle se remettra immédiatement à pousser.

Végétation: Elle produit une faible récolte l'année de semis et sa production diminue graduellement après la troisième année. Elle commence de bonne heure dans la saison et continue à pousser jusqu'à une époque avancée de l'automne.

Valeur agricole: Elle n'a qu'une importance secondaire comme plante fourragère et son emploi est assez limité. A cause de sa végétation basse, de ses feuilles courtes, serrées près du sol, elle ne peut être utilisée pour le foin. On peut en faire des pacages pour les moutons sur les terres pauvres où les graminées plus précieuses ne peuvent être cultivées avec succès. C'est là sa valeur principale. Comme elle pousse en touffes et que ses racines sont peu profondes elle ne résiste pas au piétinement, et devrait toujours être mélangée avec d'autres herbes ou aux trèfles. Semé avec le trèfle blanc, par exemple, elle donne un gazon ferme et le trèfle améliore la qualité du pâturage.

Graine: La fétuque ovine est une des herbes les moins coû. teuses; elle produit beaucoup de graine qui est facile à récolter. $\mathrm{Si}$ on la laisse trop mûrir, la graine s'éparpille. Elle est prête à être fauchée quand les épillets se rompent facilement.

Qualité de la graine: La bonne graine de commerce est de couleur paille,-un peu plus jaunâtre en général que la fétuque rouge. Elle pèse de dix à quinze livres au boisseau.

Quand je considère, écrivait Columelle, le grand art de l'agriculture, lorsque je l'envisage formant un corps d'étude d'une vaste étendue, et lorsque je passe à l'examen de toutes les parties dont il se compose, je crains de voir la fin de mes forces, avant d'avoir pu acquérir la connaissance entière.Columelle. rer siècle.

Il en est d'un champ comme d'un homme; il importe peu qu'il rapporte beaucoup, s'il codte beaucoup; alors le profit est nul. Le vrai but est de retirer l'intérêt de ses avances et de ses peines: ainsi, le premler soin doit être d'épargner la dépense.-Caton. 95-46 a. J. C. 


\title{
FÉTUQUE OVINE À FEUILLES FINES (Festuca ovina L., var. tenuifolia Sibth.)
}

\author{
Nom anglais: Fine leaved Sheep's Fescue.
}

Cette variété est généralement moins développée que la fétuque ovine ordinaire. Les feuilles en sont extrêmement fines et velues. Elle se distingue de la fétuque ovine par ses fleurs sans barbe.

Elle n'a pas de valeur spéciale en agriculture mais elle pourrait être utilisée en mélange pour les pelouses.

\section{FÉTUQUE DURETTE. (Festuca ovina., L. var. duriuscula (L.) Koch.)}

Autre nom latin: Festuca duriuscula L.

Nom anglais: Hard Fescue.

La fétuque durette n'est qu'une variété robuste de la fétuque ovine, à laquelle elle ressemble sous tous points essentiels. C'est une plante vivace à fortes touffes, dont toutes les feuilles sont enroulées comme celles de la fétuque ovine, mais dont les feuilles des rejetons de la base sont plus longues, plus épaisses et de texture plus ferme,d'où le nom de fétuque dure. Les panicules et les épillets sont un peu plus grands mais il n'existe pas de caractéristique fixe qui permette de distinguer cette plante de la fétuque ovine ordinaire. Elle a à peu près la même distribution géographique et la même valeur que cette dernière. Les sols sablonneux lui conviennent, mais on ne devrait la cultiver que lorsque la terre est trop pauvre pour les herbes de meilleure qualité. Les feuilles de la base sont assez longues et elle peut être utilisée jusqu'à un certain point comme herbe de pied pour les mélanges de foin sur les terres pauvres.

La graine de la fétuque durette est très semblable à celle de la fétuque ovine mais elle a souvent une teinte bleuâtre.

\footnotetext{
Les artistes semblent en général réserver pour eux seuls les secrets de leur art. L'agriculteur au contraire le plus habile soit à planter, soit à semer, est content lorsqu'on l'observe. Questionnezle sur les procédés qui lui réussissent, il n'a rien de caché pour vous; tant l'agriculture inspire de générosité à ses heureux sectateurs.-Xénophon, l'Economique, 434-355 a. J. C.
} 


\section{FÉTUQUE À FEUILLES DIVERSES (Festuca nelerophylla Lam.)}

Nom anglais: Various-leaved Fescue.

Description botanique: La fétuque à feuilles diverses esı une plante vivace, formant des touffes épaisses. Les tiges, qui ont de deux à quatre pieds de hauteur, sont minces et grêles. Elles sont entourées à la base de rejetons feuillés qui naissent des bourgeons, dans les gaines des vieilles feuilles, et sortent de l'ouverture de la gaine comme dans la fétuque ovine. Mais ces rejetons sont beaucoup plus nombreux que dans cette dernière. Les feuilles sont très longues, toujours enroulées et raides, mais de texture molle. Les feuilles de la tige, d'abord enroulées et raides, comme celles des rejetons de la base, deviennent bientôt plates et prennent un aspect très différent. C'est pourquoi la plante s'appelle fétuque à feuilles diverses. Les fleurs sont en panicule, souvent penchée au sommet, et généralement plus large et plus ouverte que celles de la fétuque ovine et de la fétuque durette. Chaque épillet contient de trois à neuf fleurs qui ont des barbes à moitié aussi longues ou tout aussi longues que les glumes qui les portent.

Distribution géographique: La fétuque à feuilles diverses est propre au sud de l'Europe; en Asie, elle est propre au Caucase et à l'Himalaya.

Habitat: Elle croît spontanément dans les bois ouverts et le long de leurs bords.

Culture: Elle préfère les sols bas, frais, mais elle peut supporter une longue sécheresse pourvu que la terre ne soit ni trop pauvre ni trop sablonneuse.

Valeur agricole: C'est la deuxième année après les semis qu'elle donne la récolte la plus forte; une fois avancée en âge elle forme des touffes de plusieurs pouces de hauteur semblables à des coussins. C'est une bonne herbe de pacage pour les parcs boisés, où le sol n'est pas trop sablonneux. Elle préfère les localités ombragées aux champs exposés.

Graine: Les graines que l'on vend dans le commerce sont obtenues sur des plantes sauvages dans les bois. La graine est semblable à celle de la fétuque rouge mais généralement un peu plus grosse. 



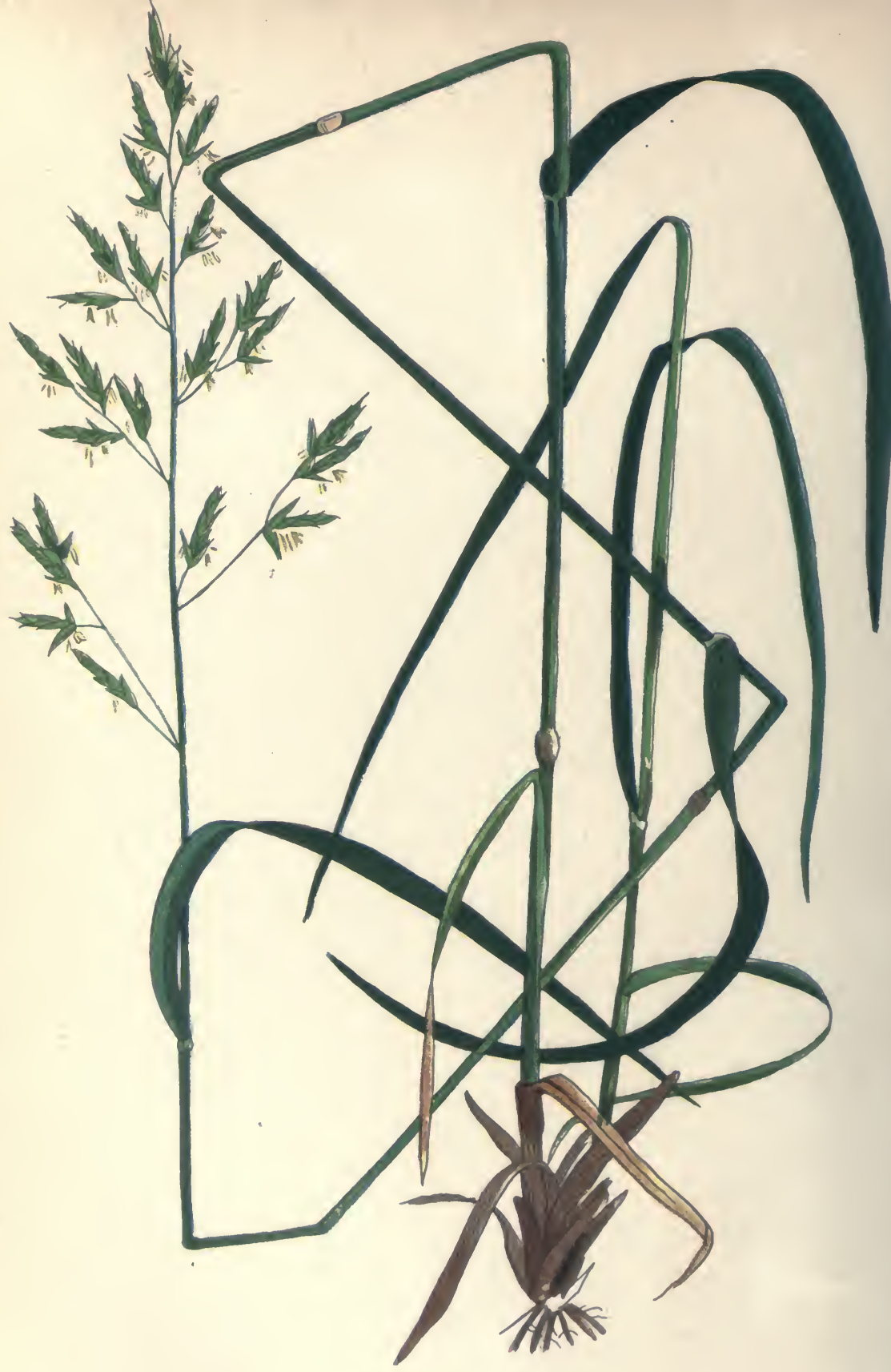

Planche 12

FÉTUQUE DES PRÉS (Festuca elatior L.) 


\title{
FÉTUQUE DES PRÉS, FÉTUQUE ÉLEVÉE (Festuca elatior L.)
}

\author{
Planche I2, Graine, Planche 26, fig. 20.
}

\section{Autre nom latin: Fesluca pratensis Huds.}

Noms anglais: Meadow fescue, Tall fescue, English Blue grass, Evergreen grass, Randall grass.

L'emploi de deux noms latins pour cette graminée a donné lieu à beaucoup de confusion. Festuca elatior signifie fétuque élevée, et Festuca pratensis signifie fétuque des prés. Les grainetiers appellent généralement la fétuque élevée Festuca elatior et la fétuque des prés Festuca pratensis, ce qui semble venir à l'appui de cette assertion répandue que la fétuque élevée et la fétuque des prés sont deux plantes distinctes en botanique. Il n'en est rien. Ce sont simplement des variétés agricoles d'une même plante dont le vrai nom latin est Festuca elatior L., tout comme la Banner et l'Abondance sont deux variétés agricoles de l'avoine, Avena sativa $\mathrm{L}$.

\section{FÉTUQUE DES PRÉS}

Description botanique: La fétuque des prés est une plante vivace, à racines longues et fortes. Ses souches sont assez courtes. Elle pousse donc en touffes mais pas autant que le dactyle pelotonné. Les tiges, qui ont de dix-huit à trente-six pouces de hauteur, sont lisses et assez grêles. La plupart des feuilles sont produites par de nombreux rejetons stériles naissant des souches, les tiges n'en portent que quelques-unes. Les feuilles sont vert foncé, assez longues et larges, de texture faible et souvent penchées. Elles sont enroulées dans les bourgeons et les jeunes tiges sont donc rondes et non pas aplaties comme dans le dactyle peletonné où les jeunes feuilles sont pliées ensemble le long de la ligne médiane. Les fleurs sont assemblées en panicules avec deux rameaux de grosseur différente à chaque nœud. Les rameaux ne s'étalent que pendant la floraison; avant et après, la panicule est étroite avec des branches dressées. Certaines graminées, notamment le Brome, ont des panicules semblables à celle de la fétuque des prés. On reconnaît cette dernière par sa panicule penchée au sommet et ses branches tournées d'un côté. Les épillets sont oblongs, souvent avec une teinte de violet. L'un d'eux contient de sept à huit fleurs dont chacune est enveloppée de deux glumes qui sont lisses et généralement arrondies. A la 
floraison les étamines et le pistil apparaissent en même temps, ce qui permet la fécondation croisée aussi bien que l'autre fécondation.

Distribution géographique: La fétuque des prés est propre à l'Europe jusqu'au cercle polaire et dans les régions tempérées de l'Asie. Elle a été introduite dans l'Amérique du nord, venant probablement de l'Angleterre où on a commencé à la cultiver vers I820.

Habitat: Comme son nom l'indique, la fétuque des prés est une herbe répandue dans les prairies de l'ancien monde. Elle croît spontanément dans les endroits incultes, le long des chemins, des voies ferrées et des rivières.

Culture: Elle vient particulièrement bien dans les terrains riches en matière organique. Les sols argileux lui conviennent très bien mais pas autant peut-être qu'au dactyle pelotonné; elle donne de bonnes récoltes sur les terres sablonneuses qui contiennent assez d'humidité et qui ne sont pas trop minces. Les sols assez frais lui conviennent mieux qu'au dactyle pelotonné, et surtout en pâturage car elle résiste mieux au piétinement que ce dernier. D'autre part, elle résiste également assez bien à la sécheresse, grâce à ses racines plutôt profondes. Généralement parlant, la fétuque des prés pousse sur presque tous les sols assez frais et pas trop pauvres. Comme elle résiste fort bien au froid elle pourrait être employée avantageusement dans bien des parties du Canada.

Végétation: Semée avec d'autres graminées ou avec le trèfle rouge, la fétuque des prés se développe assez lentement. Elle n'atteint son plein développement que deux ou trois ans après les semis. Semée seule, on peut obtenir une bonne levée la première année. Elle reste productive pendant un bon nombre d'années, surtout quand on applique à la terre une légère couche de fumier tous les ans. Elle pousse de bonne heure et est prête à être fauchée à peu près en même temps que le dactyle pelotonné ou quelques jours après.

Valeur agricole: Le foin de la fétuque des prés n'est pas tout à fait aussi bon que celui du dactyle pelotonné. C'est quand la plante est en fleurs qu'elle contient le plus de nourriture. Elle devrait donc être coupée quand toutes les fleurs sont ouvertes ou un peu plus tôt.

Laissées jusqu'après la floraison les tiges deviennent rudes et coriaces et perdent rapidement leur succulence et leur valeur nutritive. L'herbe qui vient d'être fauchée reprend promptement et donne un 
regain passable, composé principalement des feuilles des rejetons de la base. Elle est donc précieuse pour les pacages d'été et d'automne d'autant plus qu'elle supporte bien le piétinement et ne se met pas en touffes épaisses comme le dactyle pelotonné.

La fétuque des prés est bonne pour la production du lait, mais c'est dans l'engraissement des bestiaux qu'elle a le plus de valeur. Comme elle est légèrement laxative, il ne faut pas l'employer seule pour l'alimentation des chevaux d'attelage. On devrait la cultiver avec d'autres plantes fourragères, de même que le dactyle pelotonné. Semée, par exemple, avec le trèfle rouge et le mil, on obtient un mélange beaucoup plus nutritif que lorsque ces deux plantes sont seules. Semée seule, pour le foin ou le pacage, on doit mettre de quarante à quarante-cinq livres de bonne graine à l'acre.

Graine: La culture de la fétuque des prés pour sa graine est une industrie très avantageuse. Les frais de main-d'œuvre sont peu élevés car on obtient de fortes récoltes du même champ pendant au moins trois années de suite. Outre la graine produite, on a le regain que l'on peut faire paître sans nuire à la production de la graine de l'année suivante pourvu qu'on ne laisse pas paître les animaux trop longtemps ou trop tard à l'automne. On fauche quand les panicules commencent à devenir brunes et que tout le champ à l'aspect de grain en maturation. La graine coupée trop tard tombe facilement et c'est pourquoi il est nécessaire de manier une récolte avec beaucoup de soins après le fauchage. Ce que nous avons dit du fanage et du battage de la graine du dactyle pelotonné s'applique également à la fétuque des prés.

Qualité de la graine: La bonne graine de commerce a une couleur brun grisâtre, assez terne. Elle ne conserve sa vitalité que pendant un temps relativement court. On ne doit pas semer de graine qui a plus de trois ans. Semée pour la production de la graine on doit en mettre de dix à quinze livres à l'acre. Le poids du boisseau varie de douze à vingt-six livres.

Maladies: La fétuque des prés est parfois atteinte par la rouille. Cette rouille ne se montre que lorsque la récolte est coupée pour sa graine. Elle peut abîmer le regain à un tel point qu'elle gâte non seulement le pâturage mais la récolte de graine de l'année suivante en affaiblissant les plantes et en diminuant leur résistance à l'hiver. 


\section{FÉTUQUE ÉLEVÉE}

Nom anglais: Tall Fescue.

Nous avons déjà dit que la fétuque élevée est très proche parente de la fétuque des prés et ne peut être distinguée de celle-ci par des caractéristiques botaniques fixes. Elle est généralement très élevée et parfois de texture un peu plus grossière. La deuxième pousse est plus forte, ce qui en fait une bonne herbe à pacage. Des recherches faites aux Etats-Unis indiquent qu'elle est plus résistante à la rouille que la fétuque des prés.

Mais elle a des désavantages, sa maturation est inégale, certaines graines d'une panicule sont mûres tandis que d'autres sont encore molles. Il faut la couper de bonne heure pour éviter les pertes, mais alors un fort pourcentage de la graine obtenue n'est pas mûre et la qualité générale est assez pauvre.

\section{FÉTUQUE ROSEAU (Festuca arundinacea Schreb.)}

Nom anglais: Reed Fescue.

La fétuque roseau est à peu près inconnue au Canada. Elle passe parfois sous le nom de fétuque élevée et on la prend souvent pour une variété de la fétuque des prés. Cependant c'est une espèce botanique tout à fait distincte et le nom de fétuque élevée ne devrait jamais être employé pour cette herbe car il prête à la confusion.

Description botanique: La fétuque roseau est une herbe élevée qui atteint une hauteur de quatre pieds ou plus et porte beaucoup de feuilles longues et larges. Elle ressemble à la fétuque des prés mais elle est beaucoup plus grossière. Les tiges deviennent bientôt ligneuses et les feuilles raides et coriaces. Après la floraison on la distingue facilement de la fétuque des prés par sa grosse panicule aux rameaux étalés.

Distribution géographique: La fétuque roseau se rencontre en' Europe presque jusqu'au cercle polaire, dans l'Afrique du nord et l'ouest de l'Asie. 
Culture: Elle est commune le long des rives de la mer. Elle a plus de goût pour les terrains humides que la fétuque des prés. On peut donc la cultiver dans des endroits où celle-ci échouerait sûrement.

Valeur agricole: Elle n'a pas la même valeur nutritive que la fétuque des prés et les bestiaux la laissent généralement de côté au pâturage lorsqu'ils peuvent trouver d'autres herbes.

Furius Crésinus, obtenant des récoltes plus abondantes que ses voisins, fut par eux, accusé de magie. Ayant été traduit en jugement, il conduisit dans le forum, avant l'appel de sa cause, une fille robuste qu'il avait bien nourrie et bien vêtue, des ustensiles en fer de la meilleure construction, de larges bêches, des socs pesants et des bœufs vigoureux: "Voilà, dit-il, Romains, en quoi consistent mes sortilèges; mais il y manque mes méditations, mes veilles, mes fatigues, que je ne puis produire ici devant vous." A ces mots, le peuple entier se leva pour l'acquitter.-Pline, Histoire naturelle, 23-79 A. D.

D'ailleurs si elle (l'agriculture) nous offre tant de biens, elle ne permet pas qu'ils soient le prix de l'indolence; elle veut qu'on s'habitue à supporter les ardeurs de l'été, les glaces de l'hiver. De plus elle donne de la vigueur à ceux qui cultivent de leurs propres mains; quant à ceux qui en surveillent les travaux, elle en fait des hommes, en les éveillant de grand matin, en les obligeant à de longues marches.-Xénophon, $l$ Economique 434-355. J. C.

Le foin donne de la peine à faucher, continue l'Hurluberlu, bétail peine à soigner, fumier peine à le mener.... Farine peine à boulanger, dit le petit, argent peine à le gagner. Comment vous avez des besoins tous les jours et à chaque instant du jour; il n'y a que le travail qui peut les satisfaire, et vous ne voulez pas travailler. Alors mangez des cailloux, buvez à la rivière, allez tout nus, couchez à la belle étoile, puis fainéantez, reposez-vous.-Jacques Bujault.

Rien n'est changé, pourtant! Là-bas, le trèfle rouge

Brille entre l'orge épaisse et le sainfoin tremblant;

Le trèfle, où le soleil éclatant luit et bouge,

Tache la plaine en feu de son carré sanglant.

La campagne toujours a des gloires superbes,

Mais quels féconds labeurs, mais quels joyeux hymens

Si tous les bras oisifs allaient s'offrir aux gerbes,

$\mathrm{Si}$ le flot des absents remontait nos chemins!

Ahl que le déserteur s'arrête et qu'il revienne

Vers la ferme, à l'endroit où ses pères sont morts!

Du métier désappris que l'absent se souvienne!

C'est le travail des champs qui nous rendra les forts.

PAUL HAREL, Les voix de la Glèbe. 


\section{BROME INERME' (Bromus inermis Leyss).}

Planche 13; graine, planche 27, fig. 23.

Autre nom latin: Schedonurus inermis (Leyss) Beauv.

Noms anglais: Awnless Brome Grass, Brome Grass, Smooth Brome Grass, Hungarian Brome Grass, Hungarian Fodder Grass, Austrian Brome Grass, Austrian Brome Hay.

Description botanique : Le brome inerme est une plante vivace à souche rampante, qui produit de nombreux rhizomes écailleux. Ces rhizomes sont une sorte de tiges souterraines dont les feuilles sont réduites à de minces écailles. Ils sont très ramifiés, s'enracinent aux nœuds et produisent de nombreuses tiges dressées, du type ordinaire. Ils sont longs, très rampants, les tiges dressées qui en naissent sont clairsemées et les plantes ne forment pas de touffes mais des tapis verts, surtout dans les sols maigres et légers. Les tiges sont nombreuses, assez épaisses. Elles atteignent de un à quatre pieds de hauteur et portent de nombreuses feuilles étalées. Ces feuilles sont longues, larges et lisses et varient de clair à vert foncé. La panicule est généralement grosse avec des rameaux étalés dans toutes les directions. Après avoir fleuri, elle se resserre généralement parfois tout d'un côté, et ses rameaux sont penchés. Les épillets, qui ont environ un pouce de longueur, sont généralement d'un rougebrunâtre lorsque la végétation est avancée. Un épillet contient de sept à neuf fleurs et chacune de ces fleurs est enveloppée de deux glumes plus ou moins tronquées. On appelle cette herbe brome inerme parce que la glume extérieure de la fleur n'a pas de barbe. Mais il se rencontre parfois des espèces qui ont des glumes barbues tout comme les autres espèces du genre.

Distribution géographique: Le brome inerme est propre au centre de l'Europe et de l'Asie. Sa zone s'étend de la Hollande et de la France à la Chine. Bien qu'il soit très répandu, l'espèce sauvage ne se rencontre que dans de rares localités. Toutefois il a été introduit dans un grand nombre d'endroits en ces dernières années et il est maintenant assez commun dans presque tous les pays d'Europe, Il a été introduit au Canada il y a environ vingt ans et est très répandu. surtout dans les provinces des prairies.

Habitat: Le brome inerme croît spontanément dans les endroits secs, graveleux, sur les bords des rivières et sur les collines, le long des bois, plus rarement dans les prairies.

Culture: Le brome inerme n'exige pas un sol riche et lourd. Il vient bien sur des terrains ouverts, relativement pauvres, où des 


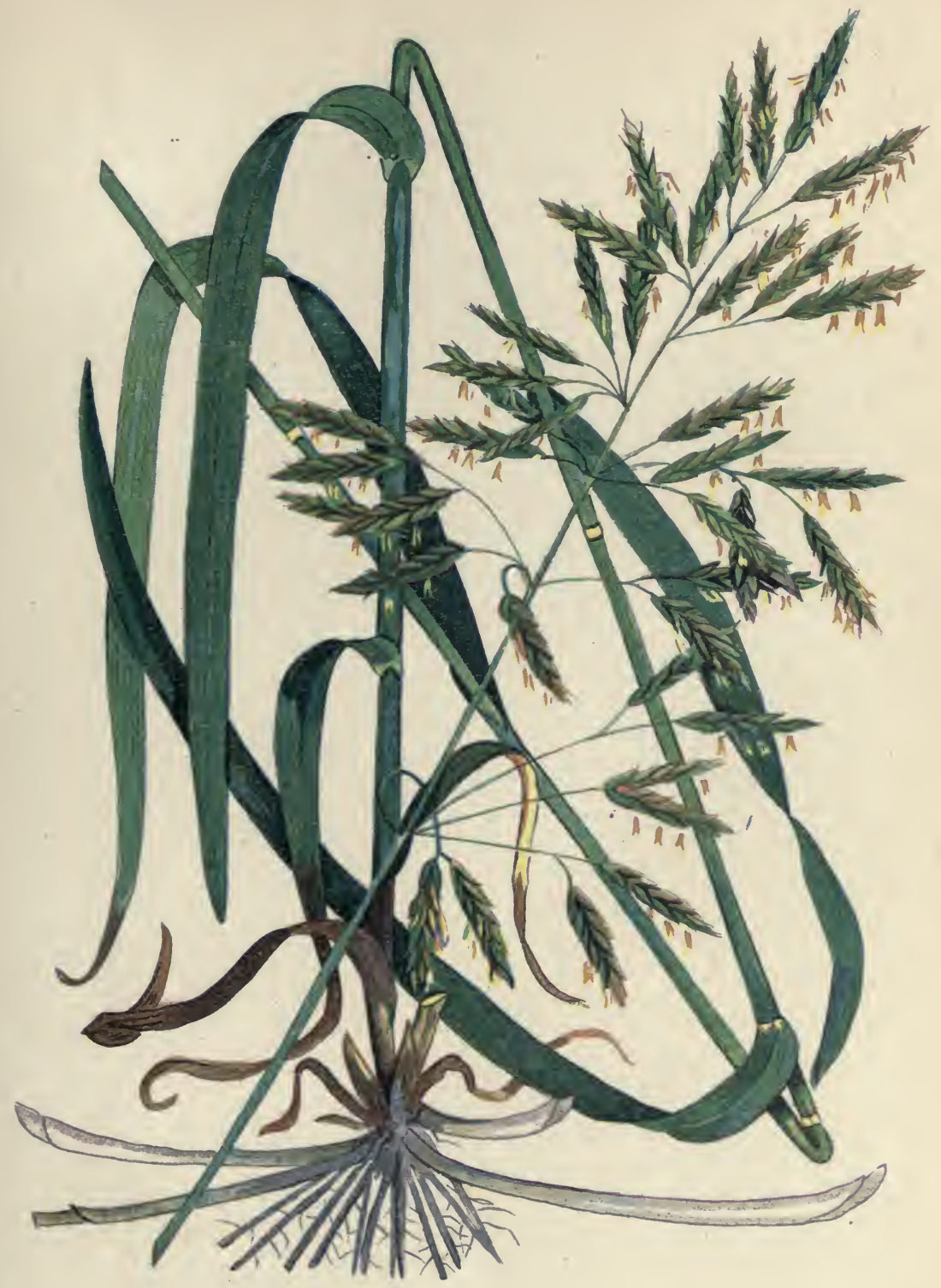

Planche 13

BRÔME INERME

(Bromus inermis Leysser:) 

herbes plus précieuses lèveraient mal. Quoiqu'il réussisse sur des sols assez frais on lui réserve de préférence ceux qui contiennent peu d'humidité. On l'estime beaucoup à cause de sa résistance à la sécheresse; dans les étés secs il produit plus de fourrage vert que toute autre herbe. Le brome inerme est surtout estimé dans les parties des provinces des prairies où la chute de pluie est faible. II convient pour l'Ouest du Canada à cause de sa rusticité et de son aptitude à supporter des changements de climat subits et considérables. On le cultive beaucoup en Hongrie où le climat ressemble assez à celui de l'Ouest canadien.

Habitudes: De même que la plupart des autres herbes vivaces, le brome inerme pousse assez lentement la première année de semis. La deuxième année, la récolte est forte et il atteint généralement son maximum de rendement la troisième année. A cause du tallage prononcé de la souche, le gazon devient bientôt trop serré et il faut labourer pour maintenir le rendement. Une fois établi il est persistant et ne disparaît qu'à la suite de façons aratoires répétées. Il se met à pousser de bonne heure au printemps et continue à produire des tiges et des feuilles jusqu'à une époque avancée de l'automne.

Valeur agricole: Son aptitude à fournir du fourrage vert, même dans un été chaud et sec, le rend précieux pour·les pâturages, mais au point de vue de la valeur nutritive il ne saurait être comparé au pâturin du Kentucky par exemple. D'autre part il supporte bien le piétinement des bestiaux et des moutons ce qui en fait une plante précieuse pour les pacages sableux et graveleux.

Fourrage: Les opinions diffèrent sur la valeur nutritive du foin, mais on peut affirmer qu'il est à peine comparable au mil de qualité moyenne. Cultivé pour son foin dans un climat sec, il vaut généralement mieux le semer sur sol nu, c'est-à-dire, sans plante-abri. A la ferme expérimentale de Indian Head on a trouvé que dix à douze livres de graine à l'acre suffisent. On obtiendrait une meilleure récolte la première année en employant plus de graine, mais mcins bonne ensuite car les racines s'épaississent et produisent un gazon trop serré. Il faut le faucher avant la floraison car il durcit et perd sa succulence et beaucoup de sa valeur nutritive. Dans des conditions favorables on peut faire deux coupes de foin en une saison, mais la deuxième coupe est assez légère et se compose principalement de feuilles. Tous les bestiaux sont friands de son foin. Il convient aussi bien à la production du lait qu'à celle de la viande. Cependant ses propriétés laxatives le rendent peu propre à l'alimentation des chevaux de travail. 
Graine: Cultivé pour sa graine, on coupe le brome inerme quand les épillets ont une nuance violet-brunâtre. Si l'on coupait trop tôt, la graine serait légère et de mauvaise qualité. On peut, sans risque, laisser la graine mûrir parfaitement avant de couper car elle est peu exposée à tomber. Les méthodes de récolte et de fanage sont les mêmes que pour les céréales.

Qualité de la graine: Les graines sont brun clair avec une teinte pourpre caractéristique; elles ont près d'un demi pouce de long et sont plates et légères. La glume porte une courte barbe au sommet mais elle est généralement sans barbe et tronquée. La graine contient ordinairement beaucoup de balle et de débris de paille cassée. La bonne graine pèse quatorze livres au boisseau.

\section{BROME DES CHAMPS (Bromus arvensis L.)}

\section{Graine, planche 27, fig. 21 .}

Description botanique: Le brome des champs est une plante annuelle ou bisannuelle, à tiges de un à trois pieds de hauteur, généralement en touffes nombreuses et à feuilles plutôt larges et molles. La couleur générale de la plante est vert grisâtre caractéristique. On la distingue facilement des autres bromes par les poils mous qui recouvrent ses parties inférieures. La panicule est forte, étalée même après la floraison, d'une teinte pourpre caractéristique. Les glumes extérieures de la fleur sont munies de longues barbes; sous tous les autres rapports les épillets sont semblables à ceux du brome inerme.

Distribution géographique: Le brome des champs est propre à l'Europe, à la Sibérie et à l'Asie Mineure. Il a été introduit dans quelques parties de l'Amérique.

Habitat: Il se rencontre dans les endroits incultes, le long des chemins et des sentiers et dans les champs, où il pousse parfois comme une mauvaise herbe.

Valeur agricole: On a essayé de cultiver cette herbe. Comme elle est de peu de durée elle ne peut être utilisée que dans de courtes rotations. Elle pousse rapidement et donne une récolte abondante. Il serait donc possible de l'utiliser en culture dérobée. Elle n'a pas encore été suffisamment essayée au Canada. 


\section{BROME GILIÉ (Bromus ciliatus L.)}

Graine, planche 27, fig. 22.

Description botanique: Le brome cilié est une plante vivace, à souche courte, et qui pousse en touffes ouvertes. Les tiges, de deux à quatre pieds de hauteur, sont assez grêles et généralement recouvertes de poils raides à la base. Les feuilles sont larges, d'un vert clair ou d'un vert presque jaunâtre, de texture molle et couvertes des deux côtés de poils mous et courts. Les panicules sont généralement unilatérales; leurs rameaux longs et penchés portent les épillets principalement aux extrémités. Les épillets sont généralement verts et se distinguent facilement de ceux de toutes les autres espèces de brome par leurs glumes qui sont ciliées de poils longs et saillants. Le lemma (voir page II) a une barbe plutôt courte et droite.

Distribution géographique: Le brome cilié est propre à l'Amérique du nord. Il est répandu dans l'est du Canada.

Habitat: Il se rencontre dans les bois humides, les taillis, le long des rivières, etc.; il préfère les endroits ombragés.

Sa valeur agricole est peu connue.

J'ai vu bien des gens n'employer que des graines de légumes préparées, et arrosées à l'avance d'un noir mélange composé de nitre et de marc d'huile; c'était pour que les cosses souvent trompeuses donnassent des grains plus gros, et plus faciles à cuire, même avec un feu modéré. J'ai vu aussi des semences de choix, triées attentivement, dégénérer pourtant, si la patience humaine ne prenait soin, chaque année, de mettre de côté une à une les plus belles. C'est la loi en ce monde; tout empire, et se trouve fatalement ramené en arrière. Ainsi le nautonnier qui, dans sa barque. fait force de rames pour remonter le cours de l'eau; si par malheur il détend les bras un seul instant, aussitôt le fleuve l'entraine en plein courant et l'emporte à la dêrive.-Virgile, Les Géorgiques, Traduction d'Henri Lantoine.

Que diriez-vous d'un homme qui sèmerait les criblures de ses céréales? Rien de favorable. Que voulez-vous donc que nous disions de notre côté, de ceux qui sèment moins encore que les criblures du foin. Nous en sommes cependant là et ne pouvons sortir d'embarras qu'en achetant dans les villes des semences qui sortent de la campagne et que nous devrions produire partout dans nos exploitations.-Joigneaux. 


\section{RAY-GRASS ANGLAIS OU VIVACE (Lolium perenne L.)}

Planche I4 A; graine, planche 27, fig. 24.

Autre nom français: ivraie vivace.

Noms anglais: Perennial Rye grass, English Rye or Ray grass, Common Darnel.

Description botanique: Cette herbe est vivace, à souche rampante, d'où naissent des touffes de tiges feuillées et de rejetons stériles. La plante pousse donc en touffes étalées qui se composent de touffes secondaires reliées par les rameaux allongés de la souche. Les tiges sont tout à fait dressées ou plus ou moins genouillées et ascendantes. Elles sont grêles, lisses et ont généralement de un à deux pieds de hauteur. Les feuilles sont généralement vert foncé et pliées ensemble dans le bourgeon. Sous ce rapport elles diffèrent de celles du ray-grass italien qui sont enroulées dans le bourgeon. L'inflorescence est en épi caractéristique, composé d'un certain nombre d'épillets aplatis disposés en deux rangs. Les épillets sont solitaires à chaque nœud et l'épi est donc assez semblable à ceux du ray-grass de l'ouest et du chiendent. Toutefois, dans le ray-grass vivace, les épillets tournent leur côté étroit vers la tige principale, tandis que dans le ray-grass de l'Ouest et le chiendent c'est le côté large qui est tourné contre la tige. Pour cette raison l'épi du raygrass vivace devient fortement aplati tandis que celui du ray-grass de l'Ouest et du chiendent est plus cylindrique. Un épillet contient de cinq à neuf fleurs et chacune d'elles est entourée de deux glumes sans barbe.

Distribution géographique: Le ray-grass vivace est propre à presque toute l'Europe, l'Afrique du Nord et aux régions tempérées de l'Asie. Il a été introduit au Canada et aux Etats-Unis et il est maintenant très répandu dans notre pays, surtout dans les provinces de l'est.

Habitat: Il croît spontanément dans les endroits incultes et les champs cultivés, sur le bord des chemins, dans les prés, le long des bois.

Culture: Il préfère les sols argileux frais et riches et fait une pousse luxuriante dans les sols qui lui conviennent. Le bon égout- 

tement du sol est le facteur principal dans sa culture. L'eau stagnante lui est désastreuse. D'autre part sur les terrains stériles et secs il donne une tige raide et coriace et disparait généralement après la deuxième année.

Climat: Bien que le ray-grass vivace puisse supporter une longue sécheresse, on ne saurait le recommander pour les districts où l'été est chaud et sec. C'est pourquoi ce ne sera jamais une plante importante pour les provinces des prairies. Comme il a une prédilection pour les climats humides, il peut être utile pour les provinces maritimes et la côte du Pacifique, surtout sur les terres lourdes et de bonne qualité.

Végétation: Le ray-grass vivace s'établit parfois assez difficilement. Toutefois ce n'est pas là une caractéristique générale; la mauvaise qualité de la graine en est souvent la cause. Etant donné des conditions favorables et une graine de bonne qualité, son développement est rapide et l'on peut obtenir une bonne végétation en quelques semaines. Il donne son rendement maximum une année après avoir été semé, mais peut continuer à produire de façon passable plusieurs années de suite.

Valeur agricole: C'est une des plus anciennes plantes fourragères de l'ancien monde. Il était cultivé en Angleterre il y a près de deux cent cinquante ans et il est fort apprécié dans les pays de la Mer du Nord. Malgré son rendement moyen et sa valeur nutritive peu élevée on le cultive de préférence à toutes les autres herbes dans certaines parties de l'Europe.

Fourrage: Le ray-grass vivace se développe rapidement et dure peu. Aussi est-il utile dans les mélanges à foin, à pâturage et à pelouses dont on cherche à tirer un rendement en attendant que des herbes plus précieuses et permanentes se soient établies. C'est immédiatement avant de fleurir qu'il renferme le plus de nourriture. Comme il est assez hâtif il devrait être mélangé à d'autres plantes à floraison hâtive, par exemple, le dactyle pelotonné.

Pâturage: On devrait commencer à le faire paître avant que les tiges soient trop développées. Dans des conditions favorables il reprend promptement et fournit de l'herbe verte jusque vers la fin de l'automne. Il supporte les mauvais traitements mieux que la plupart des autres herbes et le piétinement lui est avantageux plutôt 
que nuisible. Semé seul, trente livres de bonne graine à l'acre suffisent.

Graine: La majorité de la graine vient d'Ecosse et d'Angleterre. On devrait, pour la production de la graine, employer la meilleure terre disponible car elle se vend bon marché, et cette culture n'est avantageuse qu'à la condition d'obtenir une forte récolte. Un champ ne donne qu'une récolte de graine. On fait une coupe de foin au commencement de la saison et la deuxième récolte est réservée pour la production de la graine. En coupant trop tôt on obtient une graine légère qui ne vaut pas grand'chose car le prix se règle d'après le poids. D'autre part, si l'on coupe trop tard les semences s'égrainent et l'on en perd. Le meilleur moment est environ un mois après la floraison, quand les graines commencent à durcir.

Qualité de la graine: La graine est brun-clair, semblable à celle de la fétuque des prés. Cependant elle n'est pas aussi terne que la graine de cette dernière et a un lustre argenté. Elle est plus tronquée, plus plate et presque sans barbe.

Le poids varie, la meilleure graine pèse de vingt-cinq à trente-cinq livres au boisseau. La mauvaise graine dépasse rarement quinze livres.

\section{RAY-GRASS ITALIEN (Lolium multiflorum Lam.)}

Planche I4 B.

Description botanique: Le ray-grass italien est vivace, très semblable au ray-grass vivace par l'aspect général. Les touffes sont semblables mais les rejetons naissant de la souche du ray-grass italien sont généralement plus courts et les touffes formées par la plante sont plus épaisses. Règle générale les tiges sont plus élevées et plus délicates que celles du ray-grass vivace; elles en diffèrent également par la sensation rude qu'elles communiquent quand on les frotte à la main audessous de l'inflorescence. Un autre trait de différence avec le raygrass vivace est que, dans la jeune plante, les feuilles sont enroulées dans le bourgeon. Quand elles ont atteint leur plein développement les feuilles sont relativement larges, de texture molle et vert clair. L'inflorescence a le même aspect général que celle du ray-grass vivace, mais elle en diffère par quelques points importants. Ainsi un épillet 
contient un grand nombre de fleurs, généralement de dix à trente. Quand l'épi est développé on peut facilement distinguer l'espèce italienne de l'espèce vivace par les fleurs. Celles-ci sont entourées de deux glumes comme celles du ray-grass vivace mais la glume extérieure porte au sommet une longue barbe. Cependant certaines variétés sont sans barbe et se reconnaissent par leurs feuillles roulées quand elles sont jeunes et leurs tiges rudes vers le haut.

Distribution géographique: Le ray-grass italien est propre à l'ouest et au sud de l'Europe, au nord de l'Afrique, et à l'Asie Mineure. I' n'à été introduit que dans certaines parties de l'Amérique du nord et ne se rencontre que dans quelques localités au Canada.

Habitat: Il croît spontanément dans les prés, le long des fossés et des chemins, dans les jardins, les clairières, etc.

Culture: Le ray-grass italien est productif sur les sols humifères, sableux et calcaires et sur les marnes assez fraîches. Il ne vient pas bien sur les argiles lourdes ni sur les sols très secs. Nulle plante ne répond aussi bien à l'apport d'engrais. Il produit abondamment sur les sols engraissés par des purins. Dans la vallée de la rivière $\mathrm{P} \hat{\mathrm{o}}$, au nord de $\mathrm{l}$ 'Italie, où l'on irrigue les terrains déjà gras et riches, et sur les terrains sablonneux du voisinage d'Edimbourg, Ecosse, qui sont irrigués par les égoûts de la ville, il rend des quantités presque incroyables de fourrage. Quoiqu'il ait une origine plus méridionale que le ray-grass vivace il pousse relativement assez loin au nord. Il est douteux cependant qu'il se rende généralement utile au Canada. Peut-être aura-t-il quelque valeur sur la côte du Pacifique.

Végétation: Le ray-grass italien n'est pas d'aussi longue durée que le ray-grass vivace. Il dure peu et disparaît généralement après la deuxième année, surtout si on le laisse produire ses semences. Il se met à pousser de bonne heure au printemps; sur les terres arrosées de purin la végétation est si rapide que l'on a obtenu jusqu'à dix tonnes d'herbe à l'acre six semaines après les semis. Il perd sa succulence et sa valeur nutritive plus rapidement que le ray-grass vivace et pour cette raison devrait être coupé lorsque les épis commencent à se développer. Dans des conditions favorables on peut faire trois ou quatre coupes par saison.

Le ray-grass italien a probablement été cultivé pour la première fois dans le nord de l'Italie. Sa valeur comme plante fourragère 28550-11 
est reconnue dans la plupart des pays d'Europe. Il est l'objet d'une grande culture en Ecosse et en Angleterre.

Valeur agricole: Par sa végétation prompte et rapide, le raygrass italien est une herbe très précieuse sous un climat favorable. Cultivé seul ou en mélange avec d'autres graminées ou trèfles c'est une superbe herbe de pacage pour les assolements courts, et tous les bestiaux en sont friands. Les moutons que l'on met dans un champ ensemencé de ray-grass italien et de trèfle rouge préfèrent le premier. C'est une bonne nourriture pour les vaches laitières et il n'a pas d'effet laxatif en dépit de sa succulence. On dit qu'il n'a pas d'égal pour la production des fourrages verts et que sa croissance rapide le rend précieux dans la lutte contre les mauvaises herbes.

Graine: Les remarques faites au sujet de la production de la graine de ray-grass vivace s'appliquent également au ray-grass italien, mais ce dernier est beaucoup plus exposé à perdre ses graines et pour cette raison ne doit pas être coupé trop tard.

Qualité de la graine: La graine du ray-grass italien est semblable à celle de la fétuque des prés et très semblable à celle du raygrass vivace. On la distingue facilement de cette dernière par sa barbe longue et grêle. Sa couleur est un peu plus faible et le lustre luisant caractéristique de la graine du ray-grass vivace est beaucoup moins apparent. Le poids de la graine varie, la moyenne pour la bonne semence est de seize livres au boisseau. Elle monte parfois jusqu'à vingt-quatre livres au boisseau.

\section{RAY-GRASS DE L'OUEST (Agropyron tenerum Vas.)}

Planche 15; graine, planche 27, fig. 26.

Noms anglais: Western Rye Grass, Slender Wheat Grass, Bald Wheat Grass.

Description botanique: Le ray-grass de l'ouest est une plante vivace, à souche très courte, non rampante, comme celle du chiendent auquel il ressemble beaucoup sous tous autres rapports. Grâce au peu d'étendue de la souche les chaumes sont serrés et toute la plante pousse en touffes épaisses. Le ray-grass de l'Ouest est donc une 


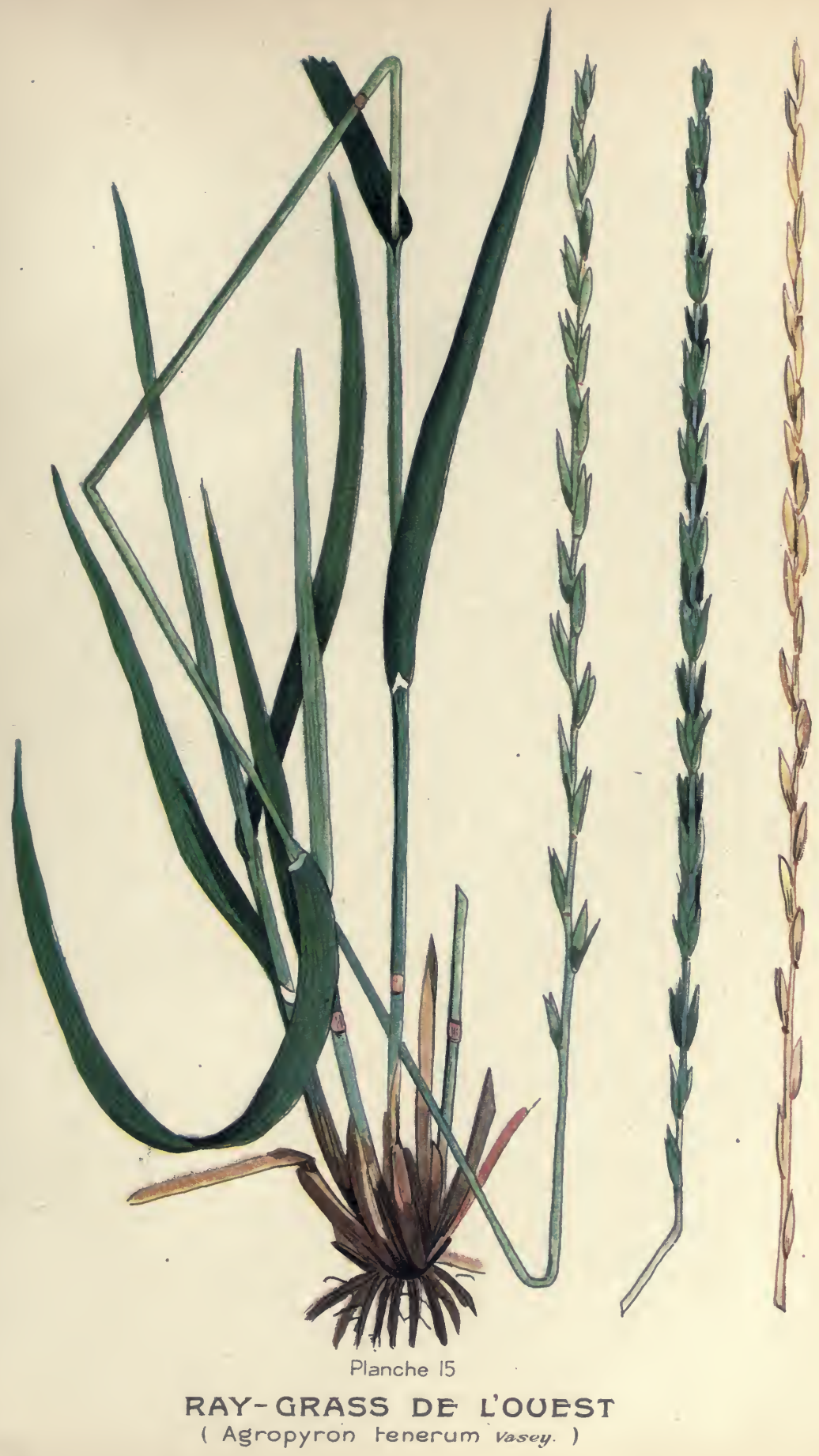



herbe touffue. En outre la souche souterraine émet un grand nombre de fortes racines, de tiges et de rejetons stériles. Ces tiges et rejetons stériles sont tout à fait droits, les premières ont de deux à quatre pieds de hauteur, les derniers varient suivant les plantes et suivant les localités. Le ray-grass de l'Ouest varie sous bien d'autres rapports. Par exemple, dans certaines plantes le feuillage est pauvre et se trouve presque entièrement à la base de la plante tandis que dans d'autres il est presque aussi riche que celui du mil ordinaire et réparti le long de la tige jusqu'au dessus du milieu de la plante. On trouve des plantes à feuilles étroites et sèches poussant à côté d'autres à feuilles larges et plutôt molles, et des plantes bleuâtres ou grisâtres à côté de plantes d'un vert éclatant. L'inflorescence longue et en épi porte, à chaque nœud, des épillets aplatis et solitaires, et ressemble ainsi quelque peu à celle du ray-grass vivace et italien. Mais dans ces deux dernières herbes le côté étroit des épillets est tourné vers la tige principale, tandis que dans le ray-grass de l'ouest (de même que dans toutes les autres espèces du genre Agropyron) le coté large des épillets est tourné vers la tige principale. Il y a donc peu de danger de confusion. En outre, dans les épillets d'une espèce d'Agropyron il y a deux glumes stériles (voir page II) tandis que les espèces du genre Lolium n'ont qu'une glume stérile. Dans le ray-grass de l'ouest les deux glumes stériles sont presque aussi longues que l'épillet entier et parfois l'enveloppent entièrement. Les épillets sont toujours étroitement serrés contre la tige principale, ce qui rend l'inflorescence resserrée et grêle, d'où le nom d'herbe grêle (Slender Wheat grass) qu'elle porte aussi en anglais. Les épillets, d'abord verts, prennent une couleur de paille à l'époque de la maturation. Un épillet contient généralement de quatre à six fleurs, chacune d'elles entourée de deux glumes. La glume extérieure est semblable, de forme et de texture, aux deux glumes stériles à la base de l'épillet. Elle est généralement sans barbe mais parfois porte une petite barbe au sommet.

Distribution géographique: Le ray-grass de l'Ouest est propre au Canada où il est répandu d'une côte à l'autre ainsi que dans les parties nord et ouest des Etats-Unis. Comme son nom l'indique, il est surtout répandu dans l'Ouest où il s'étend des régions sèches de la Colombie-Britannique au Nouveau-Mexique et à la Caroline du Sud.

Culture: Le ray-grass de l'Ouest croît spontanément dans les sols secs; pour bien faire il ne lui faut qu'une quantité d'eau limitée. 
Il ne résiste pas longtemps aux inondations répétées mais répond promptement à une irrigation judicieuse. C'est une des rares herbes qui ne souffrent pas de la présence d'une grande quantité d'alcali dans le sol. Grâce à sa racine, qui est assez profonde et très forte, il supporte sans souffrir de longues sécheresses. Il supporte également le froid excessif, et, de façon générale, résiste aux conditions climatériques défavorables mieux que la plupart des autres plantes fourragères.

Végétation: Sa culture est facile. Il pousse promptement de semis. Semé au printemps il s'établit en une saison et dans des conditions favorables peut épier vers la fin de l'automne de la même année. C'est généralement la troisième année qu'il donne son plus fort rendement, lequel dépend, jusqu'à un certain point, de la quantité de graine mise en terre.

Valeur agricole: C'est sans aucun doute la plus précieuse des graminées indigènes de l'Ouest. De même que la plupart des autres graminées fourragères il comprend bien des types différents. Les uns ne donnent qu'une petite quantité de pauvre fourrage, d'autres produisent beaucoup de foin succulent et nourrissant. Dans son état actuel, le ray-grass de l'Ouest est un mélange de types et la production est donc relativement faible. On pourrait, au moyen de la sélection et d'une culture améliorée, produire des variétés qui dépasseraient beaucoup, au point de vue du rendement et de la valeur nutritive ordinaires, les espèces que l'on cultive aujourd'hui dans les provinces des prairies.

Fourrage: Le ray-grass de l'Ouest est principalement une herbe à foin. On doit le faucher quand il commence à fleurir ou un peu avant, car c'est avant la floraison qu'il est le plus succulent et le plus nourrissant. Après cela il devient coriace, dur et tout à fait ligneux. On ne peut en tirer qu'une récolte de foin par an. C'est comme nourriture pour les chevaux qu'il a le plus de valeur. Cependant il n'est pas apprécié autant que le foin du brome inerme, peut-être parce que les types cultivés aujourd'hui portent assez peu de feuilles sur leurs tiges. faible.

Pâturage: Il a peu de valeur pour les pacages car le regain est

Semé seul on doit mettre de dix à quinze livres de bonne graine à l'acre. Il n'y a rien à gagner à faire des semis plus épais sur des sols secs. Au contraire le rendement y perd les années suivantes. 
Graine: Cultivé pour la production de la graine on doit couper quand les épillets ont une couleur paille-verdâtre; dans des conditions normales la plante arrive à cette phase de trois à quatre semaines après la floraison. On la coupe à la moissonneuse, on fane comme pour le mil et on bat dans une batteuse à grain.

Qualité de la graine: La graine est de couleur paille claire, de un-tiers de pouce à un-demi pouce de long, sans barbe ou avec une barbe courte et droite au sommet.

\section{AGROPYRE DE L'OUEST OU CHIENDENT DE L'OUEST (Agropyron occidentale Scribn.)}

Autre nom latin: Agropyron Smithii Rydb.

Noms anglais:- Western Wheat Grass, Colorado Blue Stem, Blue joint, Alkali Grass.

L'agropyre de l'Ouest est très vivace, à souche rampante, semblable à celle du chiendent. Les plantes ne poussent pas en touffes comme le ray-grass de l'Ouest mais forment un gazon ouvert, à tiges clairsemées et à rejetons feuillés, comme le chiendent. La plante entière est d'un vert bleuâtre, d'où les noms de "Blue Stem" et de "Blue joint" qu'elle porte en anglais. Les tiges, de un à quatre pieds de hauteur, sont assez fortes. Les feuilles sont relativement longues, de texture ferme, plate, ou enroulées dans les localités sèches. L'inflorescence est fortement aplatie, plus large et plus serrée que celle du ray-grass de l'Ouest. Les épillets sont à peu près deux fois plus longs et contiennent un plus grand nombre de fleurs, environ huit en général. Dans un épillet de ray-grass de l'Ouest les deux glumes inférieures sont à peu près aussi longues que l'épillet entier tandis que dans l'agropyre elles sont à moitié aussi longues.

L'agropyre de l'Ouest est propre à l'ouest du Canada. Il s'étend de la Saskatchewan aux Montagnes Rocheuses. Dans les Etats-Unis il s'étend à l'ouest à partir du Michigan et du Kansas.

Comme le chiendent, il est peu difficile sur les sols de la localité. Il se rencontre dans les plaines exposées aussi bien que sur les côtes. Bien qu'il soit très résistant à la sécheresse, on ne le trouve pas en général sur les sols très sablonneux et très secs. Il préfère les terrains riches et y fait une pousse luxuriante quand il y trouve assez d'humidité. Il réussit mieux que la plupart des autres herbes de prairie 
et d'herbage sur les sols salins, d'où le nom d'Alkali Grass qu'il porte également en anglais.

On connaît peu la valeur agricole de l'agropyre de l'Ouest. Dans quelques-uns des états de l'Ouest on l'estime surtout pour le pâturage et on le croit très nutritif. Mais son gazon devient trop compacte à la longue à cause de sa souche rampante et de sa végétation étalée, et il peut se faire qu'il ne mérite pas d'être cultivé.

\section{AGROPYRE À BARBE (Agropyron Richardsonii Schrad.)}

\section{(Autrefois appelé à tort Froment des chiens.)}

Graine, planche 27, fig. 27.

Nom anglais: Awned Wheat Grass.

L'agropyre à barbe a une souche courte et pousse en touffes comme le ray-grass de l'Ouest. Il se distingue aisément de ce dernier par les longues barbes que portent ses glumes florifères et par la disposition de ses fleurs en épi unilatéral. Il est répandu dans les provinces des prairies, particulièrement en dehors des régions semi-arides. Il est généralement plus grossier que le ray-grass de l'Ouest et convient moins que ce dernier pour faire du fourrage à cause de ses barbes longues et raides.

\section{GHIENDENT (Agropyron repens (L.) Beauv.)}

Graine, planche 27, fig. 25 .

Le chiendent est une plante très vivace, à souche très étendue, et à nombreux rejetons feuillés qui forment de grandes planches très compactes. Sa végétation est semblable à celle de l'agropyre de l'ouest mais il en diffère par sa couleur d'un vert plus clair et par ses épillets plus petits. Indigène à l'Europe il a malheureusement été introduit au Canada, dans les districts de l'Est, où il s'est solidement implanté. Malgré sa valeur relative en agriculture c'est une des mauvaises herbes les plus dangereuses, contre laquelle on doit se garder soigneusement.

Qul suivra la routine n'aura bientôt ni pain ni farine,-Bujault. 


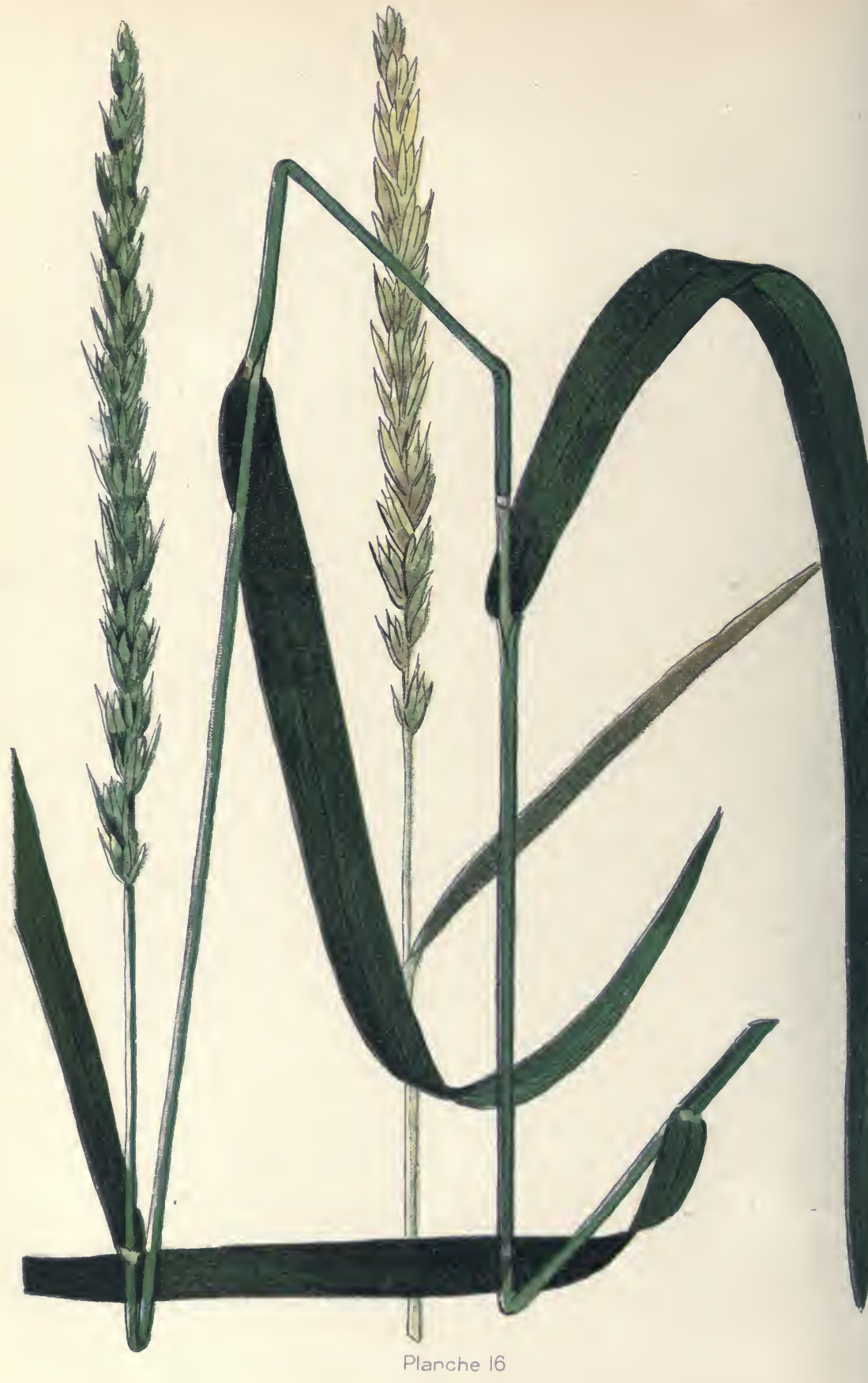

ELYME DE VIRGINIE (Elymus virginicus $L$. ) 


\section{ÉLYME DE VIRGINIE (Elymus virginicus L.)}

Planche 16; graine, planche 27, fig. 28.

Noms anglais: Virginia Lyme Grass, Bald rye Grass, Wheat Grass, Terrell Grass.

Description botanique: L'élyme de Virginie est une plante vivace, à souche très courte, et poussant en touffes serrées. Les tiges, qui ont généralement de deux à quatre pieds de hauteur, sont nombreuses, très serrées, lisses et plutôt grêles, feuillées jusqu'au sommet et souvent teintées de pourpre. Les feuilles sont longues et larges, leur couleur varie de vert clair à vert glauque. Les feuilles basses brunissent et sèchent bientôt et généralement toutes sont mortes au moment de la floraison. L'inflorescence est en forme d'épi. Les épillets ne sont pas solitaires à chaque nœud comme dans le genre Agropyron; ils sont généralement en paires, ce qui donne une inflorescence serrée et touffue. Chaque épillet porte à sa base, deux glumes stériles, il y a donc quatre glumes stériles à chaque nœud. Ces glumes sont épaisses, en forme de griffes, courbées à la base, et constituent une marque caractéristique qui permet de distinguer facilement l'élyme de Virginie des autres herbes de ce genre. Chaque épillet renferme deux ou trois fleurs dont chacune est enveloppée de deux glumes étroites. La glume extérieure n'a pas de barbe ou n'a qu'une courte barbe au sommet. Quand il y a une barbe l'épi entier ressemble quelque peu à celui du seigle; quand il n'y en a pas, il ressemble à celui du blé, d'où les noms de 'Bald rye-grass' et de 'Wheat grass' que la plante porte en anglais.

Description géographique: L'élyme de Virginie est propre à presque toute l'étendue de l'Amérique du nord. Au Canada il s'étend de la Nouvelle-Ecosse aux Montagnes Rocheuses.

Habitat: Il se rencontre le long des rivières, des bois, dans les fourrés, etc. Il est assez commun dans les bois ouverts mais rare dans les prairies. C'est pourquoi il est plus fréquent dans les provinces Maritimes, de Québec et d'Ontario, que dans les provinces des prairies.

Culture: L'élyme de Virginie supporte bien la sécheresse et les froids extrêmes et fait une pousse vigoureuse sur les sols légers et secs où beaucoup d'autres herbes donnent un faible rendement. 
Valeur agricole: Sa valeur agricole est assez douteuse. Dans les premières phases de sa végétation il est nutritif et succulent, mais il perd bien vite ces qualités en durcissant, et les feuilles de la base sèchent et brunissent rapidement. Dans un pâturage on devrait donc le faire paître de bonne heure et dans un pré à faucher on devrait le couper très vert, bien avant que les plantes aient commencé de fleurir. Il ne produit pas un regain raisonnable ce qui diminue beaucoup sa valeur comme plante à prairie ou à pâturage.

Semé seul on doit mettre quinze livres de graine à l'acre.

L'histoire de la culture du trèfle est éminemment curieuse et intéressante, et elle doit encourager les efforts, si souvent rebutés, de ceux qui cherchent à utiliser de nouveaux végétaux. Il y a deux siècles environ, l'usage de cette légumineuse était complètement inconnu et sa culture n'existait pas; celle-ci prit naissance à cette époque dans notre pays (la Belgique), et de là se répandit en Angleterre, en Allemagne et en France.

Aujourd'hui le trèfle augmente de plusieurs millions la richesse des nations, il a tué la jachère cette lèpre de l'agriculture et en se répandant de Belgique chez les autres nations, il a beaucoup contribué à donner à notre agriculture nationale la réputation dont elle jouit à l'étranger.-Mollen cité par joigneaux.

L'expérience nous apprend qu'un arpent de trèfle et de luzerne sur lequel on fait trois coupes rapporte non seulement plus de fourrage que l'arpent de pré ordinaire mais même que quarante arpents en commune soumis au pâturage toute l'année,-Mémoire présenté par la Société royale d'agriculture de France à l'assemblée nationale, le 24 octobre 1789 .

Nous n'omettrons pas une méthode de labourer que les dévastations des guerres ont suggéré dans l'Italie transpadane; les Salassiens ravageant les campagnes situées au pied des Alpes, se jetèrent sur le panic et le mil qui commençaient déjà à croltre; n'en pouvant rien tirer, ils passèrent la charrue dans les champs; la moisson n'en tut que plus abondante; et ce résultat enseigna ce qu'on appelle maintenant artrare (labourer le blé en herbe). Cela se fait quand le chaume commençant a croltre a déjà poussé deux ou trois feuilles.-Pline, Histoire Naturelle.

Sache pourtant qu'après avolr confié la semence à la terre, si tu la retournes lorsque le germe échauffé par les Influences du ciel, sera monté en herbe, c'est une nourriture que tu donnes à ton champ. c'est un engrais qui le fortifie. Si au contraire tu laisses la semence croitre librement jusqu'à la maturité du graln, il sera aussi difficlle à la terre que j'ai supposé faible, d'en produlre beaucoup, qu'à une truie languissante de nourrir beaucoup de pourceaux déjà forts.-Xénophon, l'Economique, 434-355 a. J.C. 



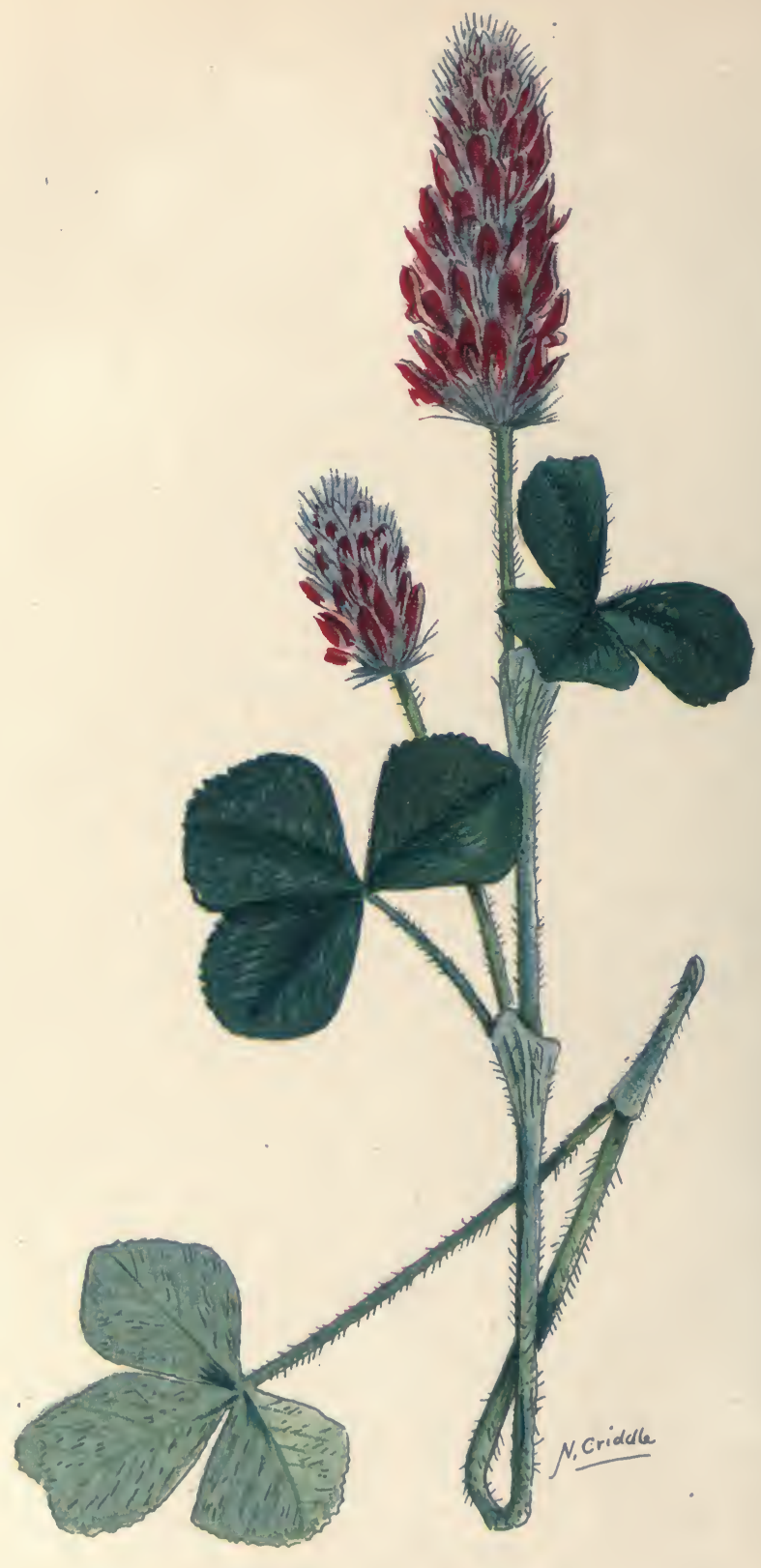

Planche 17

TRËFLE INCARNAT

(Trifolium incarnatum L.) 


\section{TREFLE INCARNAT (Trifolium incarnatum L.)}

Planche 17; graine, planche 27, fig. 29.

Autres noms français-Trèfle farouche, trèfle de Roussillon.

Noms anglais: Crimson Clover, Scarlet Clover, Italian Clover, German Clover.

Description botanique: Le trèfle incarnat est une plante annuelle, à forte racine pivotante, munie des nodosités caractéristiques à toutes les plantes légumineuses. De nombreuses tiges dressées naissent du collet de la racine et atteignent une hauteur moyenne de un à deux pieds. Elles sont de texture molle et couvertes de poils mous. Les folioles sont assez semblables de forme à celles du trè̀le rouge commun mais elles sont plus courtes, plus larges, couvertes de poils courts et dentées à la partie supérieure. L'inflorescence est en capitule allongé, et a, avant de fleurir, une teinte vert bleuâtre, singulière. Le capitule qui a de un à deux pouces de longueur à son complet développement, contient un grand nombre de fleurs dont le développement se fait graduellement de la base au sommet. Les fleurs, de couleur écarlate ou rouge cramoisi, sont un peu plus longues que celles du trèfle rouge commun.

Distribution géographique: Le trèfle incarnat est propre à l'Europe, il se rencontre en France, en Italie, en Espagne et dans la vallée du Danube. Mais dans bien des cas on se demande si la plante est réellement sauvage ou si elle a échappé à la culture. Sa culture, longtemps limitée au nord de l'Italie, au sud de la France et aux régions des Pyrénées, se répand maintenant rapidement. La limite nord de cette plante qui était approximativement le nord de l'Italie avant que l'on eât commencé à la cultiver, a reculé depuis jusqu'au sud de la Suède. En Amérique il est cultivé dans presque tous les Etats de l'Union, spécialement au sud. Au Canada il est peu cultivé et on ne le trouve guère que dans le sud de l'Ontario et la Colombie-Britannique sauf comme plante-abri dans les vergers.

Culture: Le trèfle incarnat convient bien à tous les sols pauvres. Il le doit peut-être à ses fortes racines qui permettent aux plantes de puiser leur nourriture sur une grande superficie. Il produit de fortes récoltes sur les sols légers et sablonneux où le trèfle rouge ne prendrait pas. Il faut, toutefois, pour obtenir les meilleurs résultats 28550-12 
que ces sols contiennent une quantité suffisante de chaux et d'humidité. Les sols sablo-argileux, riches en éléments nutritifs, lui conviennent particulièrement, et il y fait une végétation luxuriante si le climat est favorable. Il produit peu sur les sols lourds, argileux, froids et humides.

Végétation: Puisque le trèfle incarnat vient du sud de l'Europe, il est évident qu'il aime les climats chauds. A l'état sauvage la plante est une annuelle hivernante, c'est-à-dire que la graine germe en automne ou durant l'été et les jeunes plantes atteignent leur plein développement relativement tôt la saison suivante. Au Canada il ne résiste à l'hiver que dans les parties sud de la Colombie-Britannique. Même dans le sud-ouest de l'Ontario la graine semée tard donne des résultats peu certains. C'est donc la pratique générale de semer le trèfle incarnat de bonne heure au printemps et de rentrer la récolte la même saison. Bien qu'il préfère un climat chaud et qu'il fasse une pousse surprenante sur un sol sablonneux, le trèfle incarnat ne peut résister aux longues sécheresses. Il vient très bien dans les sols légers que l'on peut irriguer pendant la saison de végétation.

Valeur agricole: Le trèfle incarnat est très nutritif et peut être employé en foin ou en pâturage. Comme plante fourragère il est très utile dans les assolements de courte durée ou dans les vergers car il produit beaucoup de fourrage vert et les racines s'enfoncent profondément dans le sol.

Fourrage: Tous les bestiaux sont friands du foin de trèfle incarnat et l'on prétend qu'il convient tout particulièrement aux animaux dont on exige un pénible travail. Comme tous les autres trèfles c'est au moment où il est en fleurs qu'il possède sa plus grande valeur nutritive, et quand on veut en faire du foin on ne devrait pas attendre que la floraison soit passée. Les boutons ont un grand nombre de poils assez longs et qui sont doux et inoffensifs tant que les fleurs ne se sont pas ouvertes. A la floraison, ces poils deviennent raides et peuvent causer de graves indigestions. On a donc appris par l'expérience qu'il vaut mieux couper le trèfle incarnat un peu avant que toutes les fleurs se soient ouvertes. On le récolte de la même façon que le trèfle rouge.

Pâturage: Dans les régions où l'hiver est assez doux pour que l'on 'puisse semer le trèfle incarnat vers la fin de l'été, on peut le faire paître le même automne et de nouveau le printemps suivant. Lorsque 



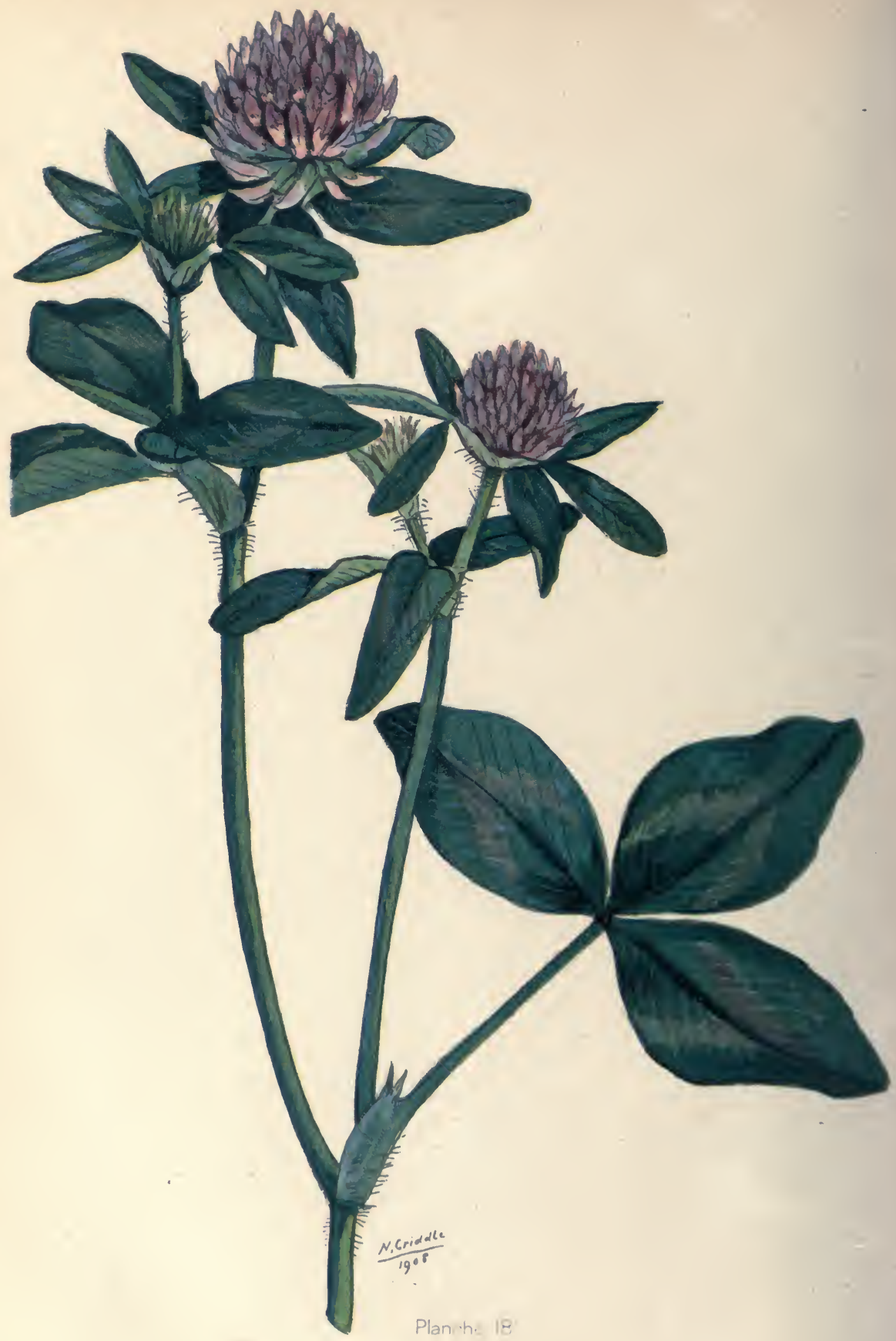

TRËFLE ROUGE

(Trifolium pratense, 
le climat, comme dans le sud-ouest de l'Ontario, exige que l'on sème au printemps, le pâturage doit être limité à une saison. Dans les parties de l'Ontario où l'on cultive le trèfle incarnat on l'emploie en pâture pour les porcs. On met de quinze à vingt livres de graine à l'acre.

Graine: Les plantes de trèfle incarnat produisent un grand nombre de capitules qui contiennent de nombreuses fleurs. Il se forme donc généralement une grande quantité de semence. Mais il faut que la température soit favorable pendant la récolte car la qualité de la graine est affectée par la pluie. Les graines tombent facilement et il est bon de couper lorsque les capitules sont humides de rosée, de manier la récolte avec le plus grand soin et de la battre aussitôt que possible. La récolte moyenne est de quatre à cinq boisseaux à l'acre.

Qualité de la graine: La graine du trèfle incarnat est beaucoup plus grosse que celle du trèfle rouge. Elle est ovoïde, bien nourrie, brun jaunâtre luisant, à teinte orangée. Le poids légal est de soixante livres au boisseau.

\section{TRËFLE ROUGE. (Trifolium pratense L.)}

Planche 18; graine, planche 27, fig. 30.

Autres noms français: Trèfle des prés, petit trèfle rouge, trèfle rouge ordinaire.

Nom anglais: Red clover.

Description botanique: Le trèfle rouge est principalement bisannuel. L'année des semis il ne se produit que des feuilles et des tiges courtes, mais pas de fleurs. La deuxième année, des fleurs se développent, la graine se forme, puis la plante meurt après avoir mûri. De même que dans la plupart des plantes bisannuelles la racine est pivotante, c'est-à-dire que l'unique racine principale s'allonge en descendant et produit de nombreuses branches latérales. C'est sur ces branches que se développent les petites nodosités rondes, en forme d'œufs, qui contiennent les bactéries nécessaires au développement de la plante. A partir de l'extrémité supérieure de la 
racine, qui est généralement élargie et que l'on appelle le collet, se forment des bourgeons plus ou moins nombreux d'où naissent des tiges feuillées. Ces tiges ont en général de six pouces à deux pieds de hauteur, elles sont nettement dressées ou ascendantes et viennent d'une base couchée; cette dernière forme est la croissance normale des tiges qui se développent de la marge extérieure du collet. Les tiges sont généralement rameuses au-dessus du milieu et les feuilles sont simples à chaque épi. Les trois folioles dont chaque feuille se compose sont oblongues ou ovoïdes et généralement marquées d'une tache blanche de forme et de grosseur variables. Les stipules, (voir page 17) attachées à la base de la queue des feuilles sont triangulaires à la base et se resserrent soudainement en une pointe alénée. Cette forme particulière est une caractéristique qui permet de distinguer facilement le trèfle rouge du trèfle intermédiaire ou trèfle Zigzag* (Trifolium medium L.) auquel il ressemble beaucoup et avec lequel on le confond assez souvent. Les stipules du trèfle Zigzag sont étroites dans toute leur longueur. Les fleurs du trèfle rouge sont en capitule épais d'environ un pouce de diamètre à son entier développement. Elles varient de rouge vif à pourpre mais sont parfois blanches.

Biologie de la fleur: $\mathrm{Si}$ on isole le trèfle rouge au moment de la floraison de façon à ce que les insectes ne puissent avoir accès aux fleurs, aucune graine ne se formera car le pollen ne peut être transporté d'une plante à l'autre que par les insectes. Les bourdons qui visitent les fleurs pour en obtenir le nectar montrent beaucoup d'activité dans ce transport du pollen. Les fleurs de trèfle rouge sont très sensibles au toucher; lorsqu'un bourdon en quête de miel introduit sa langue jusqu'au fond d'une fleur entièrement développée il touche les étamines et le pistil qui, à ce contact, se projettent brusquement au dehors de la fleur, comme mus par un ressort, et frappent le corps de l'insecte que les étamines saupoudrent de leur pollen. Notons ici que le pistil se projette un peu plus loin que les étamines et frappe l'insecte avant elles. Ce fait, en apparence insignifiant, a son importance, car il faut, pour que le pistil d'une fleur de trèfle rouge soit fécondé, qu'il reçoive le pollen d'une autre fleur. En d'autres termes, le trèfle rouge est auto-stérile. Le pollen d'une fleur est incapable de féconder le pistil de sa propre fleur. Mais en touchant l'insecte le premier, le pistil se recouvre du pollen que l'insecte a déjà recueilli sur d'autres plantes, et ainsi la fécondation croisée est assurée.

\footnotetext{
- Le trèfle Zigzag ainsi appelé a cause de la courbure en zigzag de ses tiges a des feuilles beaucoup plus étroites que le trèfle rouge. C'est une plante vivace, commune en Europe où elle pousse le long des bois et dans les clairieres. Le trefle rouge vivace de Simpson de l'Ile du Prince-Edouard et le treffle ehiendent des Provinces Maritimes appartiennent à cette espèce.
} 
Règle générale, l'insecte porte assez de pollen recueilli sur les différentes plantes pour que le pistil puisse en recevoir. Mais il arrive parfois qu'une seule visite ne suffit pas. Aussi, pour donner aux fleurs toutes les occasions d'être fécondées, la nature a fait en sorte que chaque fleur de trèfle rouge soit visitée à maintes reprises par des insectes. Dans la luzerne chaque fleur n'a qu'une chance d'être fécondée (voir page 126), car les étamines et le pistil, après l'explosion de la fleur, ne retournent pas à leur position primitive. Mais une fleur de trèfle rouge a beaucoup d'occasions, car le pistil et les étamines ne se lèvent qu'un instant, après quoi ils retournent à leur position primitive. Ils ne perdent pas leur sensibilité après la première visite d'un insecte. Une deuxième visite aura le même effet. Et tant que le pistil reste en état de recevoir le pollen, il a des chances d'être convenablement fécondé.

A l'exception de certains papillons, les bourdons sont les seuls insectes dont la langue soit assez longue pour atteindre le nectar au fond du tube de la fleur. Les apiculteurs n'ignorent pas que l'abeille ordinaire ne peut récolter le miel du trèfle rouge parce que sa langue est beaucoup trop courte. Cependant elle est également très utile dans la fécondation, car, si elle ne peut atteindre le miel, elle peut arriver au pollen qu'elle ramasse pour en faire du pain pour les abeilles. En ce faisant elle vient en contact avec le pistil et contribue ainsi à la fécondation.

Après que la fleur est fécondée, il se forme une petite gousse droite qui contient une graine. Quand cette graine est bien mûre, la partie supérieure de la gousse, qui sert de couvercle, tombe et la graine peut sortir.

Le trèfle rouge et toutes les autres espèces du genre Trifolium se comportent d'une manière assez singulière après avoir fleuri. Leurs fleurs ne tombent pas, mais elles restent fanées sur le capitule pendant toute la saison, ce qui donne aux capitules mûrs leur aspect brun caractéristique. Cette singularité fait qu'il est facile de distinguer le genre Trifolium du genre Medicago, les fleurs de ce dernier n'étant pas persistantes. Les gousses de la luzerne et d'autres espèces de Medicago sont visibles pendant la maturation tandis que celles du trèfle rouge et d'autres espèces de Trifolium ne sont pas visibles.

Distribution géographique: Le trèfle rouge est propre à l'Europe, au sud-ouest de l'Asie, à certaines parties de la Sibérie et au nord de l'Afrique.

Historique: Il a été introduit dans la culture à une date relativement tardive. On le cultivait aux quinzième et seizième siècles 
en Italie et en Espagne. Il a été introduit d'Espagne en Hollande au seizième siècle et de là s'est propagé en Angleterre pendant la première moitié du dix-septième siècle. Le nom anglais est dérivé du mot hollandais "Klafver". Il a été introduit dans l'Amérique du nord pendant la dernière décade du dix-huitième siècle.

Culture: Venant de la zône tempérée le trèfle rouge convient surtout pour les régions où les étés ne sont pas trop chauds ni les hivers trop rigoureux. Malgré ses racines assez profondes, la plante souffre des sécheresses longues et persistantes. Il lui faut assez de pluie pendant la saison de végétation pour qu'elle puisse fleurir toute la saison. Comme le trèfle rouge est assez cosmopolite on en a tiré un grand nombre d'espèces adaptées aux différents climats. Pour un climat du nord comme celui du Canada, la rusticité est le facteur le plus important. Le trèfle rouge du Chili et les autres variétés qui ont pris naissance dans les pays à climat doux sont invariablement détruites par l'hiver au Canada, même dans le sud de notre pays. Il importe donc de se procurer de la graine qui vienne du nord. On devrait toujours donner la préférence à la graine cultivée en Canada, parce que la graine produite au pays donne en général les meilleurs résultats.

Sol: Le trèfle rouge peut être cultivé avec succès sur bien des sols, mais particulièrement sur les sols gras, argilo-sableux, qui contiennent une certaine quantité de chaux et beaucoup d'humus. Les terres sablo-argileuses lui conviennent également, surtout celles qui ont un fond de chaux, mais en règle générale il préfère les sols plus lourds. Il vient même sur les argiles lourdes pourvu que le sous-sol soit ouvert. De même que pour la luzerne, le développement du trèfle rouge dépend, dans une large mesure, de la nature du sous-sol. Il faut que celui-ci soit ouvert et bien égoutté. L'eau stagnante à la surface ou près de la surface lui nuit beaucoup. Les terres saturées d'eau ne renferment pas assez d'air pour entretenir la respiration des racines et sont en mauvais état physique pour affronter les gels et les dégels alternatifs du printemps. On sait que l'eau augmente de volume en se changeant en glace; or les sols qui sont saturés d'eau à la surface se soulèvent en gelant, entraînant avec eux les parties supérieures des plantes. Or, comme les racines inférieures sont ancrées dans le sous-sol et qu'elles ne peuvent prendre part à ce soulèvement, elles s'allongent et parfois se rompent. Les effets désastreux des gels et des dégels alternatifs montrent bien qu'un des facteurs les plus essentiels au succès dans la culture du trèfle est le bon égouttement du sol. 
Végétation: Le trèfle rouge, qui est bisannuel, consacre sa première saison de végétation à développer ses racines et à se fortifier pour affronter l'hiver. Il produit donc une forte racine pivotante qui, dans des conditions favorables de sol et de température, pénètre à une profondeur considérable. Les parties aériennes de la plante se composent d'abord de quelques tiges courtes et droites qui portent des feuilles mais pas de fleurs. Plus tard dans la saison il se produit des rejetons courts et feuillés, généralement couchés à plat sur le sol, et que l'on appelle les touffes d'hiver. En même temps la racine pivotante se met à se contracter jusqu'à ce que sa longueur originale soit réduite d'au moins dix pour cent. Comme l'extrémité de la racine est fermement ancrée dans le sol il en résulte que les parties aériennes de la plante sont tirées en bas. Ce procédé, que l'on observe dans d'autres plantes, comme les carottes et les panais, a évidemment pour but de mettre les tiges et les feuilles en contact étroit avec le sol où elles sont mieux protégés contre la gelée et les vents. Au commencement du printemps de la deuxième année, les branches des touffes d'hiver forment des tiges florifères, lesquelles, si elles ne sont pas coupées ou broutées, produisent des graines et meurent vers la fin de l'automne. Ainsi la grande majorité des plantes de trèfle sont bisannuelles. Il existe cependant des types de trèfle rouge qui montrent une tendance manifeste à vivre plus longtemps, surtout si on empêche les plantes de monter à graine par des fauchages répétés ou un pâturage continuel. Le mieux connu de ces types vivaces est le trèfle Mammouth ou grand trèfle rouge.

Valeur agricole: Aucune plante fourragère n'est aussi précieuse à l'agriculture que le trèfle rouge. Il le doit, non seulement à sa valeur nutritive, presque sans égale, mais aussi à sa valeur fertilisante. On sait en effet qu'il engraisse les sols et en améliore la texture. Nulle autre plante fourragère légumineuse ne l'égale sous ce rapport.

Fourrage: C'est quand il est en fleur que le trèfle rouge a le plus de valeur nutritive. Il devrait être fauché au moment où les capitules commencent à brunir. Coupé tard, les tiges sont coriaces, elles ont perdu leur succulence et la valeur du foin est bien diminuée. La qualité du foin dépend dans une grande mesure du mode de fanage adopté. Des manutentions peu soigneuses causent une perte de feuilles. L'exposition à la pluie ou aux fortes rosées décolore le foin, lui enlève son arome et réduit sa valeur nutritive. De même, une exposition trop prolongée au grand soleil le rend moins nourrissant. Dans le 
fanagc du trèfle rouge on devrait employer des méthodes dans lesquelles le séchage se fait autant que possible par les vents.

Pâturage: Il est peu de légumineuses qui surpassent le trèfle rouge comme plante à pâturage. Tous les animaux de la ferme en sont friands. Comme les jeunes plantes de trèfle sont très délicates et qu'il est nécessaire qu'elles soient vigoureuses au commencement de l'hiver on fera bien, dans les provinces des prairies, de ne pas faire paître le trèfle rouge l'année où il a été semé. Dans certaines parties de l'Ontario où la végétation peut être un peu trop forte vers la fin de la première année, on fait généralement pâturer les champs. On se règle pour cela sur les conditions. Un pâturage trop prolongé en automne ou trop au ras du sol par des moutons, peut affecter la récolte suivante. La deuxième année on peut commencer à faire pâturer au début du printemps et continuer jusqu'à la fin de l'automne. Quand on met des bovins et des moutons dans un champ de trèfle rouge la météorisation est à craindre, surtout si l'herbe est humide de rosée et que les animaux se mettent à paître avec l'estomac vide.

Semis pour prairies et pâturages: Dans l'Ontario on sème toujours le trèfle rouge sur sol ombragé, c'est-à-dire avec une planteabri. Dans les provinces des prairies la plante-abri fait plus de mal que de bien et l'on ne doit pas s'en servir. Tel est, du moins, le résultat des essais faits aux fermes expérimentales du Manitoba et de la Saskatchewan, particulièrement à Indian-Head. Dans un climat sec ou sur des sols secs la plante-abri, par l'eau qu'elle enlève au sol, dépouille les plantes plutôt qu'elle ne les protège. Il en résulte que les plantes de trèfle sont faibles au commencement de l'hiver et plus sujettes à être détruites par la gelée. Quand on sème le trèfle rouge seul on met de dix à douze livres de graine à l'acre.

Graine: Il se produit très peu de graine de trèfle rouge au Canada à l'exception du sud-ouest de l'Ontario. La question de savoir si un champ de trèfle rouge peut être utilisé pour la production de la graine dépend des conditions. Si celles-ci sont favorables on laisse généralement monter à graine le champ entier ou certaines parties du champ. Le succès dépend de bien des facteurs. Si, vers la fin de l'été, lorsque la maturation s'opère, le temps est pluvieux, il est beaucoup plus difficile d'obteni: de la bonne graine que lorsque le temps est chaud et sec. Les sols froids et lourds qui donnent une végétation luxuriante conviennent mal en général pour la production de la graine. Les meilleurs sont les sols sablo-argileux qui contiennent une abondance 
de chaux et une bonne provision de potasse et de phosphate. Une bonne levée de trèfle améliore la qualité du foin mais ne produit qu'une faible quantité de graine. Une récolte relativement claire donne un rendement plus élevé et la graine produite est de meilleure qualité. Pour la production de la graine on doit donc semer plus clair que lorsque l'on veut établir un pré à faucher ou un pâturage.

La première coupe de trèfle rouge donne un faible rendement de graine parce que les bourdons qui fécondent les fleurs sont moins nombreux au commencement de l'été que vers la fin. Pour cette raison on coupe la première pousse pour la convertir en foin ou on la fait pâturer, et on laisse la deuxième pousse produire de la graine. En coupant ou en faisant paître la première pousse on arrête la végétation des mauvaises herbes et l'on obtient un regain plus propre.

Pour la production de la graine on coupe le trèfle rouge lorsque la plupart des capitules ont bruni et que les tiges commencent de sécher. A ce moment la majorité des graines sont mûres et ont une couleur foncée. Si l'on coupait plus tôt, on aurait une plus forte proportion de graines non mûres et retraites, la vitalité serait faible et la couleur et l'aspect généralement inférieurs. On peut couper à la faucheuse ordinaire. Les capitules et les fleurs se détachent facilement au cours des manutentions peu soignées, surtout si le temps est sec et chaud. Le battage doit se faire par un temps froid et sec car il est difficile de séparer les graines des gousses lorsqu'il fait humide.

Qualité de la graine: La graine de trèfle rouge ordinaire de commerce a une couleur variée, quelques graines sont jaunes, d'autres pourpres, d'autres claires à un bout et foncées à l'autre. Dans quelques échantillons c'est la couleur claire qui domine; dans d'autres, au contraire, les graines foncées sont les plus communes. C'est une croyance populaire que les graines de couleur claire ne sont pas bien mûres et que les échantillons qui renferment une forte proportion de ces graines sont de qualité inférieure. C'est là une erreur. Toutes les graines provenant d'une même plante ont la même couleur générale. Dans quelques plantes toutes les graines sont jaunes, dans d'autres elles sont violet-foncé et dans d'autres encore de deux couleurs uniformes. Les graines d'un jaune clair sont tout aussi mûres que les graines d'un pourpre-foncé. Le mélange des couleurs dans un échantillon commercial n'indique donc pas que les graines ne sont pas uniformément mûres; il indique simplement que l'échantillon se compose de graines de différents types et que chaque type 28550-13 
a sa propre couleur. Quoique les graines plus foncées soient plus recherchées, il ne faudrait pas juger de la valeur d'après la couleur car les types de plantes les plus désirables peuvent avoir des graines d'un jaune pâle.

La bonne graine de trèfle rouge devrait être lustrée et ne contenir qu'une faible proportion de graines retraites, ou imparfaitement développées. Le poids légal est de soixante livres au boisseau.

Impuretés: La graine de trèfle rouge contient trop souvent des graines de mauvaises herbes dangereuses. Les plus à craindre sont l'herbe à poux, le silène noctiflore, le silène enflé, le chardon du Canada, la bardane et le plantain. D'autres herbes moins dangereuses sont la prêle, le chou-gras, la persicaire, le plantain pâle, la sétaire jaune et l'oseille de brebis.

Maladies: Le trèfle rouge a beaucoup d'ennemis. Les insectes causent parfois de grands dégâts dans les cultures de cette plante. Les charançons et autres insectes se nourrissent de ses feuilles et le ver perceur de la racine attaque les racines. Ce dernier, qui, heureusement, n'est pas très répandu au Canada mange la partie centrale des racines; c'est un fléau sérieux partout où il abonde. La mite $\mathrm{du}$ trèfle qui se nourrit des jeunes graines cause fréquemment de grandes pertes. Partout où elle se trouve en nombre menaçant il faut faire paître le trèfle ou le couper de bonne heure.

Diverses sortes de cuscutes vivent sur le trèfle rouge. Les sortes que l'on trouve dans les graines commerciales varient suivant l'endroit où la semence a été produite. La cuscute la plus fréquemment introduite au Canada est celle qui vient avec la graine de trèfle rouge du Chili. Cette cuscute (dont le nom latin est Cuscuta racemosa Mart. var. chiliana Engelm.) est très différente de la cuscute de la luzerne (voir page 132). Il est vrai que toutes les cuscutes se ressemblent mais leurs effets sur les plantes sont très différents. La cuscute du Chili, par exemple, très répandue dans les districts producteurs de graines de l'Ontario, semble incapable de s'établir et ne fait donc presque pas de mal. Mais d'autres espèces de cuscutes peuvent s'introduire et nuire à la culture du trèfle.

Fauchez votre foin au bon moment, surtout n'attendez pas qu'il soit trop tard. Fauchez avant que la graine ne soit mare.-Caton. 95-46 A.J.C. 


\section{VARIÉTÉS DE TRĖFLE ROUGE.}

Le trèfle rouge se compose de milliers de types différents aux points de vue botanique, biologique et pratique. Au point de vue botanique on distingue deux types principaux que l'on pourrait appeler le type américain et le type européen. Le dernier a des tiges lisses tandis que les tiges du premier portent des poils raides et hérissés. Ces poils sont une cause d'infériorité pour le type américain car ils rendent le foin poussiéreux. Chaque type compte de nombreuses variétés qui diffèrent l'une de l'autre à certains points de vue pratiques tels que le rendement, la rusticité, la maturité, le tallage après la coupe et la durée. La plupart des variétés sont simplement des variétés géographiques. Les noms différents - trè̀le du Chili, d'Angleterre, de Suède, de France, du sud et du nord de la Russie,- - ne signifient pas que les variétés sont distinctes au point de vue botanique; ils se rapportent seulement à l'origine de la graine ou à l'adaptation des espèces à certains pays.

Le grand trèfle rouge ou trèfle Mammouth appelé "Cow grass" en Angleterre est une des meilleures variétés connues. On l'a souvent appelée Trifolium medium L. mais ceci n'est pas exact. Le Trifolium medium $\mathrm{L}$. est une espèce differente que l'on distingue facilement du trèfle Mammouth par de nombreux traits essentiels (voir page I08). Comme son nom l'indique, le trèfle Mammouth est une grande variété de trèfle rouge. Il est plus vivace et c'est pourquoi il a été appelé Trifolium pratense var. perenne c'est-à-dire trèfle rouge vivace. Les tiges sont longues, grossières, généralement étalées. Les feuilles n'ont pas la tache blanche caractéristique du trèfle rouge ordinaire. Ce n'est pas là cependant un trait distinctif car on voit souvent des pieds de ce dernier qui n'ont pas de tache. Le grand trèfle rouge est beaucoup plus tardif, il fleurit de dix jours à trois semaines plus tard que le petit trèfle rouge, et ne peut généralement être coupé qu'une fois par saison. Il exige le même sol et le même climat que le petit trèfle rouge mais à cause de ses racines plus fortes et de sa tendance à être vivace il est plus apte à pousser dans des conditions adverses. Son foin, plus grossier, est moins apprécié des bestiaux; d'autre part sa pousse plus vigoureuse en fait une meilleure plante pour le sol.

Il est impossible de séparer la graine du grand trèfle rouge de celle du trèfle rouge ordinaire. 


\section{TRÈFLE BLANC (Trifolium repens L.).}

Planche 19; graine, planche 27, fig. 3 I.

Autre nom français: Trèfle rampant.

Noms anglais: Dutch Clover, White Clover.

Description botanique: Le trèfle blanc est une plante vivace qui pousse en plaques à l'état naturel. Les tiges sont rampantes et émettent des racines aux noeuds. Une seule plante peut ainsi se répandre sur une grande superficie. Les tiges rampent à la surface du sol et émettent des feuilles droites et des capitules de fleurs, tous deux munis de longues queues. Le jour et dans des conditions normales les trois folioles de chaque fleur s'étalent au même niveau et donnent à la plante sa nuance vert foncé bien connue. Mais vers le soir la plante prend un aspect tout différent. Les feuilles à trois découpures semblent avoir disparu et sont remplacées par des feuilles plus petites, d'une couleur gris bleuâtre. Quand le soleil se couche, les deux folioles latérales se replient vers la tige principale, se tournant en même temps de façon à se trouver finalement face à face. La foliole du centre se courbe, tournant sa face inférieure gris bleuâtre vers le haut de façon à couvrir les deux folioles latérales. Lorsque les folioles latérales sont face à face et que la foliole du centre les recouvre on dit que la feuille dort. On observe des mouvement semblables dans toutes les espèces communes de trèfle et dans un grand nombre d'autres plantes. Ils sont surtout visibles dans le Shamrock.

Les capitules du trèfle blanc naissent des parties basses de la tige, ceux du trèfle d'alsike des parties supérieures. Les capitules du trèfle blanc sont plus petits que ceux du trèfle d'alsike et les fleurs sont d'un blanc pur. De même que dans d'autres espèces, les fleurs commencent à s'ouvrir à la base du capitule. Comme les fleurs brunissent et restent brunes sans tomber, le même capitule peut être blanc à sa partie supérieure et brun vers le bas.

Biologie de la fleur: Le trèfle blanc est fécondé par des insectes qui portent le pollen d'une fleur à l'autre. Les graines ne se développent que lorsque les fleurs ont été fécondées par le pollen d'une autre plante. Les fleurs sont riches en miel et comme ce miel ne peut être atteint que par les abeilles ordinaires, le trèfle blanc est précieux aux apiculteurs. Les gousses mûres sont oblongues et renferment je trois à cinq graines. 


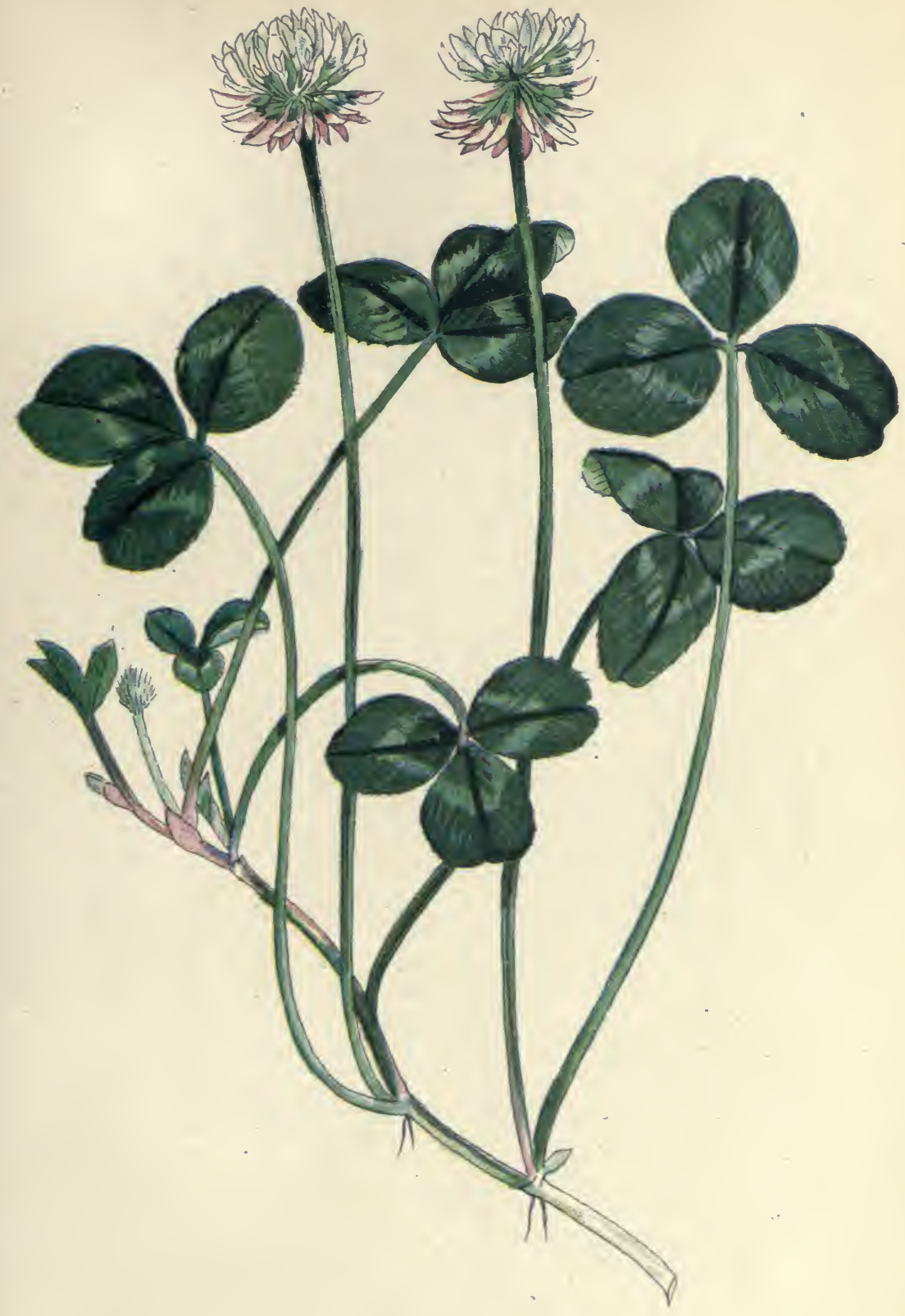

Planche 19

TRĖFLE BLANC

(Trifolium repens $L$.) 

Distribution géographique: Le trèfle blanc est propre à toute l'Europe, au sud-ouest de l'Asie, à la Sibérie et au nord de l'Afrique. On prétend également qu'il est indigène au Canada et aux EtatsUnis mais ceci est douteux. Les localités où il se rencontre naturellement au Canada sont celles dans lesquelles il a échappé à la culture et le fait que toutes les espèces indigènes de Trifolium ne se rencontrent qu'en Colombie-Britannique et qu'elles forment un groupe très différent des espèces européennes indique que le trèfle blanc n'est pas propre à ce pays mais qu'il a été introduit par les premiers colons.

Historique: Le trè̀le blanc a d'abord été cultivé en Hollande, d'où son nom anglais "Dutch Clover"; on le cultive actuellement dans toute l'Europe et pratiquement dans toute l'Amérique du nord.

Culture: Il croît spontanément dans les prairies et les pâturages, le long des chemins et des cours d'eau, dans les vallées et les montagnes. Il vient sur une grande variété de terres sableuses, graveleuses, franches et argileuses. Il prefère les sols frais, bien égouttés, qui renferment de la chaux et de l'humus. Il redoute les sols trop lourds ou qui contiennent de l'eau stagnante.

Climat: Le trèfle blanc se plaît particulièrement dans les climats plutôt humides et modérément chauds.Il résiste aux froids excessifs pourvu que les autres conditions soient favorables. Il peut également supporter une longue sécheresse à condition que le sol rontienne assez d'humidité. Il est vrai que la plupart des racines sont assez superficielles et la végétation est donc affectée par la sécheresse mais la racine principale pénètre à une profondeur considérable et les plantes survivent lorque le sous-sol contient assez d'eau. Au Canada c'est dans les Provinces maritimes, dans les régions des Grands Lacs et sur la pente du Pacifique que le trèfle blanc réussit le mieux. Il est peu adapté aux provinces des prairies.

Végétation: Une fois qu'il a pris racine le trèfle blanc couvre rapidement le sol. Les tiges rampantes émettent beaucoup de rejetons et de racines et forment promptement de grandes plaques. Les capitules produisent de nombreuses graines qui tombent à terre et conservent longtemps leur vitalité. Quelques graines tombées sur le sol peuvent en peu d'années faire des plaques de trèfle d'une grandeur surprenante. Cette facilité de propagation explique la présence du trèfle blanc dans des endroits où il n'a pas été planté et fait voir également pourquoi on le considère comme étant indigène au Canada. 
Le développement est lent en temps sec mais la plante se remet promptement à pousser lorsque la pluie tombe. Les plantes qui germent de bonne heure au printemps fleurissent généralement l'automne de la même année. La deuxième année et les années suivantes elle produisent beaucoup de capitules, qui, lorsque les plantes sont tenues en échec par le pâturage, continuent à reparaître presque toute la saison.

Valeur agricole: Le trèfle blanc ne peut guère être utilisé comme plante à foin à cause de sa végétation basse. Il convient principalement pour les pâturages, soit seul, soit comme herbe de pied dans les mélanges. Il a une valeur toute spéciale pour ce dernier but. Il recouvre promptement le sol et fournit un pacage très nourrissant, recherché de toutes les sortes de bétail. Il résiste bien au piétinement et le pâturage au ras du sol, loin de lui faire du mal, provoque une nouvelle végétation. Lorsque l'humidité est suffisante il donne un bon pacage du printemps à la fin de l'été. Conıme il est vivace il convient tout particulièrement pour les pacages permanents et les pelouses. On sème de six à huit livres de graine à l'acre.

Graine: La graine du trèfle blanc se vend plus cher que celle de la plupart des autres espèces de trèfle et dans les saisons raisonnablement humides sa production est une industrie très avantageuse mais dans les sécheresses prolongées la récolte est faible. La production moyenne est quatre à cinq boisseaux à l'acre.

Cultivé pour la graine on le fait pâturer généralement au printemps; parfois on fait une coupe de foin au commencement de la saison pour encourager la production des capitules. Comme la floraison s'étend sur une longue période, la maturation est parfois inégale. La récolte de graine devrait être coupée lorsque la plupart des capitules sont devenus foncés. Les manutentions exigent le plus grand soin.

Qualité de la graine: La graine du trèfle blanc est d'aspect rouge jaunâtre; tout échantillon se compose d'un mélange de graines rouges et jaunes. On croit généralement que la bonne graine est d'un jaune clair et que la semence rouge est vieille et a une faible faculté germinative, mais des essais de graines rouges et jaunes, prises dans des échantillons commerciaux, ont fait voir qu'il n'existe pas de différence essentielle dans la germination, pourvu que les graines rouges soient bien nourries et que leur développement soit normal.

Le poids légal de la graine est de soixante livres au boisseau. 



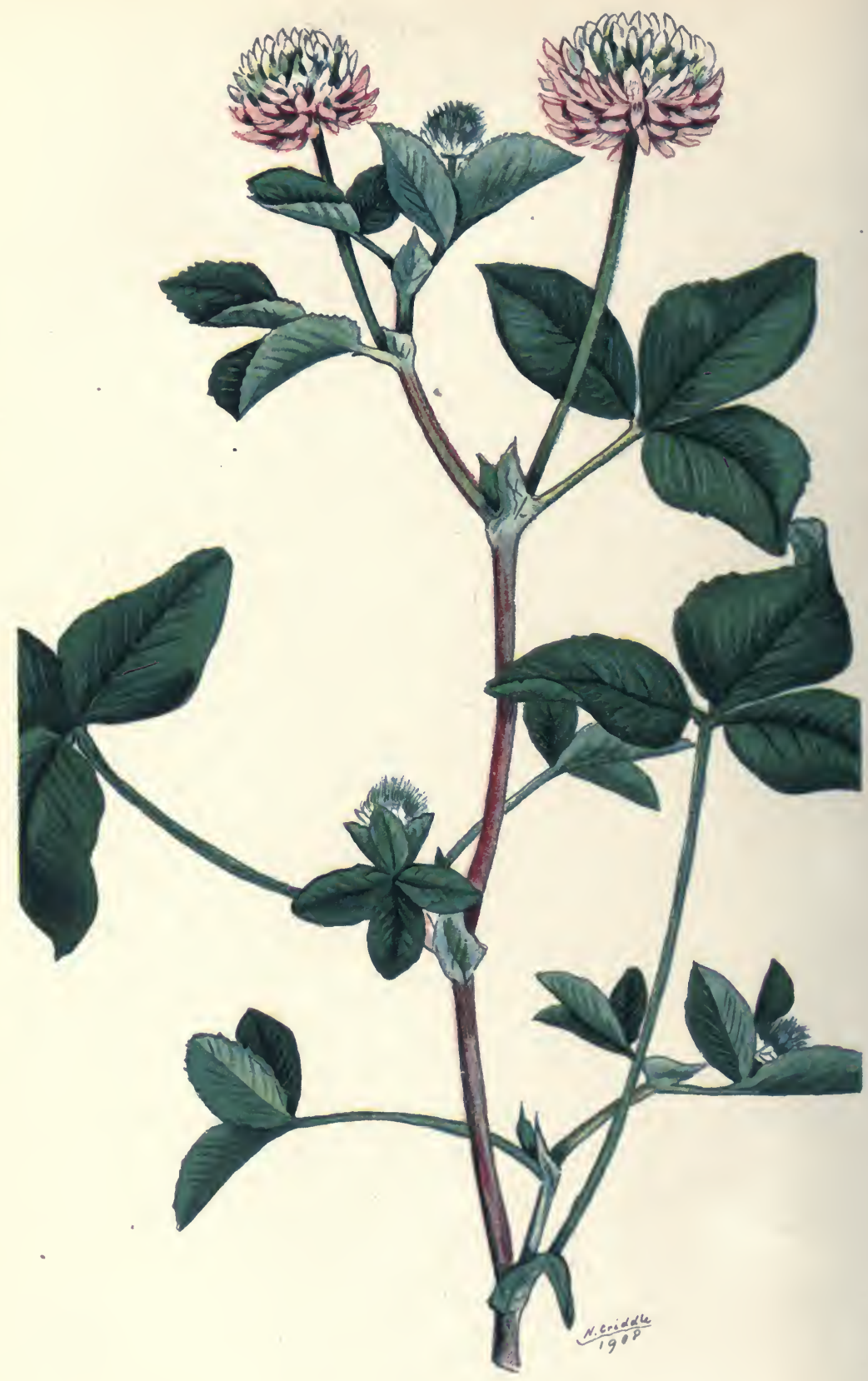

Planche 20

TRĖFLE D'ALSIKE

(Trifolium hybridum L.) 


\section{TRÈFLE D'ALSIKE (Trifolium hybridum L.)}

Planche 20; graine, planche 27, fig. 32.

Autre nom français: Trèfle hybride.

Nom anglais: Alsike clover.

Description botanique: Le trèfle d'alsike est vivace, à tiges dressées ou généralement ascendantes qui ne s'enracinent pas à la base comme celles du trè̀le blanc. Elles atteignent généralement une hauteur de un à deux pieds et sont rameuses dans leur partie supérieure. La plante entière est sans poils, et, pour cette raison, de couleur plus claire que le trèfle rouge. Les folioles sont plus courtes et relativement plus larges. Les capitules ont des queues beaucoup plus longues que ceux du trèfle rouge et ressemblent plus à ceux du trèfle blanc. Ils en diffèrent cependant par ce fait qu'ils naissent aux parties supérieures des tiges, généralement près du sommet des branches. En couleur les fleurs tiennent le milieu entre le trèfle rouge et le trèfle blanc. Il ne faudrait pas en conclure cependant que le trèfle d'alsike est un hybride, comme son nom latin le donnerait à penser; cette conclusion serait tout à fait erronée. La couleur des fleurs varie généralement de blanc à rose dans le même capitule car elles sont blanches lorsqu'elles sont jeunes et deviennent graduellement roses. Dans toutes les espèces de trèfle, les fleurs commencent à se développer à la base du capitule. Comme les fleurs de trèfle d'alsike sont roses lorsqu'elles sont entièrement développées et blanches lorsqu'elles sont jeunes, il est facile de comprendre pourquoi le capitule est généralement rose au bas et blanc au sommet.

Biologie de la fleur: De même que tous les autres trèfles, le trèfle d'alsike est fécondé par les insectes, mais il livre son nectar aussi bien à l'abeille ordinaire qu'au bourdon. La première a donc plus d'importance dans la fécondation de ce trèfle que dans celle du trètle rouge. Une fleur visitée par une abeille se comporte de la même façon que celle du trèfle rouge; comme celle-ci elle reste stérile lorsqu'elle ne reçoit pas le pollen d'un autre fleur. La fécondation croisée est essentielle entre les différentes plantes.

Après s'être ouvertes les fleurs persistantes brunissent et s'inclinent plus ou moins. Elles tombent facilement, surtout lorsque les capitules sont secs. Dans le trèfle rouge les fleurs mûres sont plus solidement attachées aux capitules. La gousse du trèfle d'alsike est 
plus longue que celle du trèfle rouge; elle fait un peu saillie au-dessus du sommet de la fleur et contient de deux à quatre graines.

Distribution géographique: Le trèfle d'alsike est propre à l'ancien monde, il se rencontre dans le nord de l'Italie, au nord de la Suède, dans le centre de la France, au sud et au centre de la Russie. On le trouve également dans le sud-ouest de l'Asie et dans certaines régions du nord de l'Amérique. Il croît spontanément le long des chemins et des cours d'eau, dans les prairies fraîches, sur les pentes des montagnes.

Historique: Le trèfle d'alsike tire son nom d'une petite paroisse du centre de la Suède, appelée Alsike où on a commencé à le cultiver il y a environ cent ans. Son emploi s'est généralisé en Europe vers le milieu du dix-neuvième siècle et sa culture est maintenant répandue dans tous les pays européens, à l'exception des pays les plus au sud. Au Canada il n'est guère cultivé d'une façon appréciable que dans les provinces de l'Est. On le trouve souvent échappé à la culture dans les provinces maritimes, Québec, Ontario et la ColombieBritannique.

Culture: Le trèfle d'alsike préfère les localités où l'humidité est abondante dans l'air et dans le sol. Les sols qui lui conviennent le mieux sont les sablo-argileux frais ou les argiles. De même que le trèfle rouge il se développe lentement. Le centre de l'Ontario renferme des terres argileuses et graveleuses, riches en chaux, qui lui conviennent tout particulièrement. Il vient bien dans des terrains trop humides pour le trèfle rouge ordinaire. Sur des terres mal drainées où ce dernier échouerait sûrement, le trèfle d'alsike réussit pourvu que les autres conditions soient favorables.

Climat: Le trèfle d'alsike n'a pas été cultivé avec succès au Manitoba et en Saskatchewan. La cause peut en être l'hiver rigoureux ou la saison sèche ou encore les deux. Il semble, d'après l'expérience acquise en Europe, que cet échec dans les provinces des prairies doit être attribué principalement à la sécheresse de l'été. La sécheresse prolongée empêche les jeunes plantes de se développer convenablement avant l'arrivée des froids et les expose à être détruites par l'hiver. Même les vieilles plantes bien établies souffrent plus de la sécheresse que le trèfle rouge. La cause doit évidemment en être attribuée au manque de profondeur des racines du trèfle d'alsike qui ne lui permet pas d'aller chercher de l'eau dans le sous-sol; dans les district secs et bien irrigués on obtient de bons rendements. 
Végétation: Dans des conditions favorables le trèfle d'alsike fleurit vers la fin de l'automne de l'année où il a été semé. Généralement cependant, à l'arrivée de l'hiver, les plantes sont aussi développées que celles du trèfle rouge. Le printemps suivant la pousse reprend relativement tard et les plantes ne fleurissent pas aussi tôt que celles du trèfle rouge ordinaire. Généralement elles sont de deux semaines en retard sur ce dernier et fleurissent à peu près en même temps que celles du grand trèfle rouge. Quand on fauche pour le foin le regain se développe rapidement mais il est rare que l'on puisse faire une deuxième coupe.

Valeur agricole: Dans les prés à foin on cultive généralement le trèfle d'alsike en mélange avec le trèfle rouge et certaines graminées comme le mil et l'agrostide; cultivé seul le trèfle d'alsike est sujet à verser à cause de sa végétation étalée. En mélange les herbes et les trèfles plus forts soutiennent l'alsike et le foin produit est de meilleure qualité. Le mélange généralement employé dans l'Ontario se compose de deux livres de trèfle d'alsike, huit de trèfle rouge et quatre de mil à l'acre. Règle générale, on emploie le petit trèfle rouge ordinaire quoiqu'il soit plus hâtif que les deux autres. Si on coupe le mélange pour en faire du foin au moment où le trèfle rouge est à l'état voulu, le trèfle d'alsike et le mil ne sont pas prêts. D'autre part, si l'on attend que ces derniers soient prêts, alors le trèfle rouge a perdu de sa valeur nutritive. Pour le foin le trèfle d'alsike devrait être fauché quand il est en pleine floraison ou un peu plus tard, c'està-dire lorsque les premières fleurs ont commencé à brunir. Le fauchage tardif n'affecte pas la valeur du foin d'alsike autant que celle du trèfle rouge car les plantes d'alsike restent plus longtemps vertes et succulentes. Généralement on ne peut enlever en une saison qu'une récolte de foin. La récolte se fait de la même façon que pour le trèfle rouge. Règle générale cependant le fanage est plus difficile, surtout si le trèfle d'alsike est cultivé en mélange avec des herbes.

Pâturage: Quoique les animaux ne soient pas aussi friands du trèfle d'alsike que du trèfle rouge, le premier cependant est très estimé pour les pâturages à cause de sa grande valeur nutritive. On peut commencer à le faire paître dès que les plantes sont bien établies et on ne devrait jamais attendre qu'elles aient fleuri. Par une température favorable un champ de trèfle d'alsike pâturé de bonne heure durera beaucoup plus longtemps que si on laissait les plantes atteindre un plus fort développement. Comme il est vivace il $28550-14$ 
convient mieux que le trèfle rouge pour les pâturages permanents. Cultivé seul on doit semer de six à huit livres de graine à l'acre.

Production de la graine: Dans des conditions favorables la production de la graine de trèfle d'alsike est une industrie avantageuse. Les plantes produisent un grand nombre de capitules et les fleurs sont à peu près sûres d'être fécondées car les bourdons et les abeilles s'y mettent tous les deux. Le sol doit contenir une quantité raisonnable de chaux, de potasse et de phosphate et pas trop d'humidité. L'excès d'humidité produit une végétation luxuriante et les plantes sont exposées à verser. Il en résulte que la production de la graine est relativement faible et de mauvaise qualité. On obtient généralement la graine de la première récolte. Parfois on fauche les champs ou on les fait paître au commencement de la saison. La maturation en est retardée mais les plantes sont mieux fournies et produisent une plus grande quantité de graine. Pour prévenir la verse qui affecte la production de la graine on sème parfois jusqu'à six livres à l'acre et l'on obtient de bons résultats. Ces semis épais sont recommandés par les grainetiers pour les sols lourds et relativement frais.

Le trèfle d'alsike destiné à la production de la graine est prêt à être coupé lorsque la plupart des capitules sont bruns et que les fleurs s'enlèvent aisément. Comme les fleurs tombent séparément lorsque la graine est mûre, la récolte doit se faire avec grand soin. Le danger est d'autant plus grand que les capitules sont plus secs et il est donc bon de couper lorsque les plantes sont humides de rosée. Pour les mêmes raisons les autres manutentions de la récolte doivent être faites avec soin. On bat de préférence en temps sec. Règle générale on ne prend qu'une récolte de graine sur un champ de trèfle. Parfois cependant on peut faire deux ou même trois récoltes de graine en autant d'années.

Qualité de la graine: La graine de commerce est jaunâtre ou verdâtre ou parfois très foncée, en masse. Chaque échantillon renferme des graines de différentes sortes. Les unes sont d'un vert jaunâtre, d'autres presque noires, mais la masse à une couleur qui tient le milieu entre ces deux extrêmes. De même que dans le trèfle rouge ces couleurs différentes n'indiquent pas que certaines graines soient plus mûres que d'autres. Le trèfle d'alsike, de même que le trèfle rouge, se compose d'un grand nombre de types qui ne diffèrent pas seulement par l'aspect général des plantes mais aussi par la couleur de la graine. Si l'on ramasse toutes les graines d'une seule plante, on trouve qu'elles sont toutes de la même couleur, quel que soit leur âge. 
Dans certaines plantes les graines sont d'un vert jaunâtre, dans d'autres elles sont noires et dans d'autres encore vertes à une extrémité et d'un noir verdâtre à l'autre. La couleur mélangée d'un échantillon ordinaire ne vient donc pas de ce que la graine a été recueillie à différentes phases de son développement mais du fait que différents types ont été mélangés ensemble.

Le poids légal du boisseau est de soixante livres.

Impuretés: Règle générale, le trèfle d'alsike contient plus de graines de mauvaises herbes que le trèfle rouge; c'est parce que la graine du trèfle d'alsike est prise dans la première récolte tandis que celle du trèfle rouge provient de la deuxième. Les graines de mauvaises herbes dangereuses que l'on rencontre dans l'alsike sont le silène noctiflore, le silène enflé, le chardon du Canada, la cameline, la bardane et le plantain. D'autres mauvaises herbes également communes sont la prêle, le chou-gras, l'oseille de brebis, la camomille puante et le mouron. On y trouve aussi le lupin noir qui ne peut être considéré comme une mauvaise herbe dangereuse mais qui, en grande quantité, est une impureté peu désirable.

\section{MÉLILOT BLANC OU TRÈFLE D'ODEUR (Melilotus alba Desr.)}

\section{Graine, planche 27, fig. 33 .}

Autre nom français: Mélilot de Sibérie.

Noms anglais: White sweet clover, Bokhara Clover.

Description botanique: Le trèfle d'odeur est bisannuel, à forte racine pivotante et à nombreuses tiges feuillées. Ces dernières, généralement très rameuses et très étalées, atteignent une hauteur de deux à cinq pieds. Lorsque les plantes sont jeunes elles ressemblent à celles de la luzerne mais on les en distingue facilement par leur arome particulier. Les feuilles sont semblables à celles de la luzerne mais ne sont pas aussi nombreuses. Quand les plantes sont en fleurs on les reconnaît facilement par leurs fleurs blanches, en longues grappes étroites semblables à des épis. Les fleurs sont petites, de construction plus simple que celles du trèfle rouge et de la luzerne; elles sont plus ouvertes et par conséquent plus accessibles aux insectes qui ramassent le pollen. La gousse est brune quand elle est mûre et ne renferme généralement qu'une ou deux graines. Comme celle de la minette elle tombe sans se rompre.

La plante a un parfum caractéristique d'où le nom de trèfle d'odeur-ce parfum se remarque surtout lorsque l'on fait faner la tige et les feuilles pour en faire du foin. 
Distribution géographique: Le trètle d'odeur est propre à l'ancien monde où il se rencontre à peu près dans toute la zone tempérée. Il est probable qu'il a été introduit au Canada avec les premiers colons car il est maintenant répandu sur toute l'étendue du continent. Il est commun partout au Canada, surtout dans les provinces de l'Est.

Gulture: Le trèfle d'odeur se trouve généralement dans les endroits incultes le long des chemins, des voies ferrées, sur le bord des rivières et dans les champs cultivés. Il pousse aisément sur presque tous les sols et se maintient là où aucune autre plante ne pourrait s'établir. Cette aptitude à pousser presque partout et sa faculté de se resemer de lui-même en font une mauvaise herbe partout où il n'est pas désiré. Il vient bien dans la plupart des climats et se maintient dans des conditions très adverses.

Valeur agricole: Dans la première phase de sa végétation, le trèfle d'odeur est succulent, mais dès qu'il fleurit les tiges deviennent ligneuses et perdent leur succulence. Son odeur repousse le bétail qui ne le mange que lorsqu'il ne peut se procurer autre chose. Le lait et le beurre produits par des vaches qui se nourrissent de trèfle d'odeur vert ont un goût particulier que n'aiment pas la plupart des gens. En outre, comme la production de foin n'est pas élevée, le trèfle d'odeur fait une pauvre plante fourragère. Sa valeur principale réside dans le fait qu'il enrichit le sol et améliore son état physique. De même que les autres plantes légumineuses, il porte, sur ses racines, des nodosités remplies de bactéries qui ramassent l'azote. On dit que ces bactéries sont semblables à celles de la luzerne et il semble y avoir des preuves concluantes à l'appui de cette assertion. Quoi qu'il en soit, elles se comportent de la même manière que ces dernières et peuvent être employées pour l'inoculation des luzernières. Six à huit livres de graine à l'acre suffisent.

Graine: Les graines de trèfle d'odeur sont communes dans les échantillons commerciaux de trèfle rouge et de luzerne. On les rencontre parfois dans le trèfle d'alsike. Elles sont jaunes, ternes, très semblables à celle du lupin jaune desquelles elles se distinguent par leur plus forte grosseur et une marque légère en "V" partant de la ccatrice.

Rappelez-vous toujours que l'agriculture est une science de localités; que ce qui convient ici ne convient pas là; qu'il n'y a rien d'absolu, que tout est relatif. Souvenez-vous queles principes changent ou se modifient de commune à commune, de ferme à ferme, et d'un champ à un autre. N'oubliez pas que les préceptes varient par le sol, comme avec la température, et les usages établis par le commerce.Bujault. 



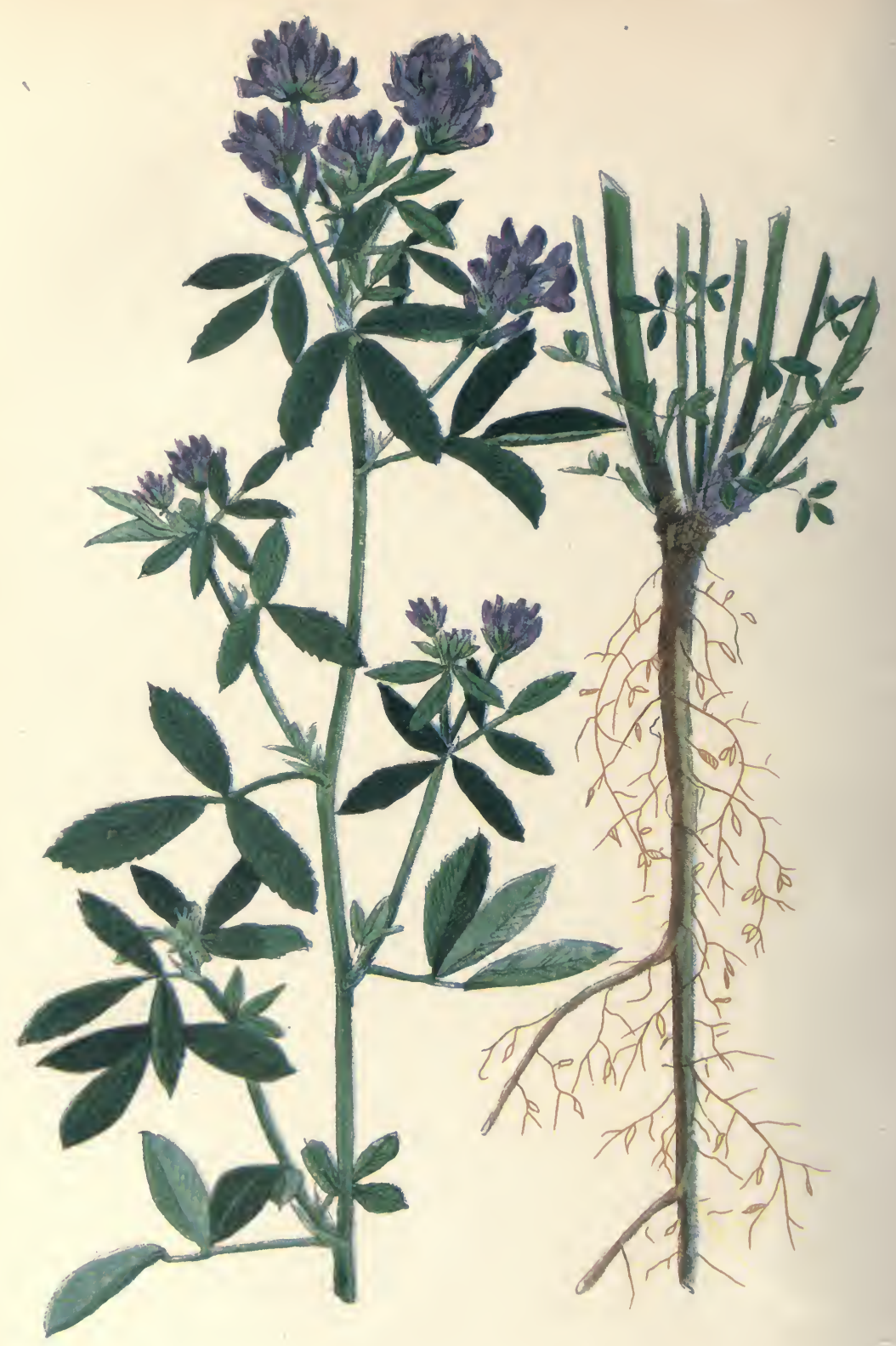

Planche 21

LUZERNE

(Medicago sativa L.) 


\section{LUZERNE (Medicago sativa L.).}

Planche 2 I; graine, planche 27, fig. 34 .

Autre nom français: Luzerne commune.

Noms anglais: Alfalfa, Lucerne.

Description botanique: La luzerne cultivée est une plante très vivace qui, dans des conditions favorables, peut se maintenir trente ans ou plus. Elle a une racine pivotante typique, c'est-à-dire que le système radiculaire se compose d'une forte racine principale d'où naissent des racines secondaires latérales. Comme il n'y a pas de rhizomes ou de racines rampantes, toutes les branches aériennes partent de la partie supérieure de la racine qui, généralement, fait saillie au-dessus du sol et que l'on appelle le collet. En vieillissant le collet se fend en deux branches ou plus dont les extrémités supérieures sont libres et forment une sorte de touffe, parfois d'une circonférence étendue. La racine principale qui, dans une vieille plante de luzerne, a un pouce ou plus d'épaisseur et est assez ligneuse, descend jusqu'à une profondeur considérable si le sol le permet. Sur la racine se trouvent les nodosités typiques des plantes légumineuses. Elles se rencontrent sur les ramifications plus fines et sont groupées ensemble en masses irrégulières. Les tiges, extrêmement nombreuses dans les vieilles plantes, ont généralement de deux à trois pieds de hauteur au moment de la floraison. Règle générale elles sont peu rameuses, surtout lorsque la récolte est serrée. Elles sont arrondies au-dessous, plus ou moins anguleuses au sommet et généralement lisses. Les feuilles qui sont alternes (c'est-à-dire solitaires à chaque nœud et éparpillées le long de la tige) se composent de trois folioles comme celles du trèfle rouge. Les folioles sont assez étroites, deux ou trois fois plus longues que larges, et nettement dentées dans leur partie supérieure. Celle du milieu a une queue courte qui n'existe pas dans les espèces cultivées de Trifolium. Parfois on trouve des feuilles à quatre ou cinq folioles mais pas aussi souvent que dans le trèfle rouge.

Biologie de la fleur: Les fleurs sont rassemblées en grappe courte et parfois unilatérale. Chaque grappe contient de dix à vingt fleurs pourpres de la forme ordinaire aux légumineuses, décrite page I6. Elles sont fécondées par les insectes et surtout par certaines espèces d'abeilles. Dans toutes les plantes légumineuses fécondées 
de cette manière, les étamines viennent en contact direct avec le corps de l'insecte. Prenons, par exemple, un bourdon qui visite une fleur de luzerne. Comme le nectar est au fond de la fleur il lui faut plonger sa langue jusqu'au fond du tube. Quand il arrive en contact avec la partie inférieure de la fleur, ce contact fait l'effet d'une pression exercée sur la détente d'un fusil. La grappe des étamines est disposée comme un ressort qui se détend brusquement à la moindre touche, projetant en haut la partie supérieure des étamines et du pistil. Ainsi, un insecte assis sur une fleur sera frappé et son corps saupoudré de pollen. Quand il visite une autre fleur, la même chose se produit, cette fois le pistil vient en contact avec le pollen qui recouvre le corps de l'insecte, ce pistil est donc fécondé et l'insecte reçoit une nouvelle couche de pollen. Il est évident que la fécondation croisée doit se produire fréquemment. Mais comme un insecte visite probablement beaucoup de fleurs d'une même plante et voyage d'une plante à l'autre, une plante peut être fécondée par son propre pollen aussi bien que par le pollen d'un autre. On n'a pas encore démontré quel mode de fécondation est le plus avantageux, si c'est l'auto-fécondation ou la fécondation croisée. Si l'insecte qui visite la fleur n'était pas assez fort pour l'ouvrir, cette fleur ne serait pas fécondée car elle ne peut se détendre d'elle-même.

La production de la graine dépend donc largement des insectes. La température est également un facteur, les fleurs sont presque insensibles par un temps froid et pluvieux, tandis qu'elles répondent promptement à la plus légère irritation par un temps ensoleillé. Dans le trèfle rouge ordinaire les étamines et le pistil reprennent graduellement leur position originale et comme leur élasticité n'est pas affectée par une ou deux visites, il y a toujours une chance que la fécondation soit bien faite. Dans la luzerne il n'y a qu'une occasion; si la première visite de l'insecte n'a pas d'effet, les fleurs ne produisent pas de graines. Après que le pollen a été déchargé, le pistil ne revient pas à sa position originale, son extrémité supérieure reste fermement appliquée à l'étendard de la fleur. Il se développe donc en un fruit courbe, bien qu'il soit parfaitement droit tant qu'il reste enfermé dans la fleur. Sa courbure, qui commence avec l'explosion de la fleur, augmente avec son développement, et lorsque le fruit est mâr, il a la forme d'une coquille tordue.

Distribution géographique et historique: La luzerne est originaire de l'Asie, probablement des régions sud-ouest de ce pays; on la cultive en Perse depuis un temps immémorial. C'est peut-être 
la plante fourragère la plus ancienne de l'univers. Son fourrage est très estimé pour les chevaux; le nom qu'elle porte en Perse veut dire fourrage à chevaux. De la Perse elle fut introduite en Grèce vers l'an 500 avant J.-C. d'où elle se répandit en Italie. Elle fut importée de l'ouest de l'Europe par voie de l'Afrique du nord. Les Arabes apportèrent la plante avec eux en Espagne au dix-septième siècle; de l'Espagne elle s'introduisit en France. On la cultive actuellement dans tous les pays européens à l'exception des pays septentrionaux. Elle a été introduite par les Espagnols au Mexique d'où elle s'est répandue aux Etats-Unis de l'Ouest et à l'Amérique du Sud, et par les Anglais et autres coloniaux aux parties est de l'Amérique du Nord. Elle est actuellement cultivée sur toute l'étendue des Etats-Unis. Au Canada on ne la propage que dans de petites superficies. Le sud de l'Ontario et le sud de l'Alberta sont les deux seuls districts où elle soit l'objet d'une grande culture.

Origine du nom: Le mot alfalfa, sous lequel la luzerne est connue en Amérique, est une version espagnole de l'arabe "Alfacfacah" qui veut dire "La meilleure sorte de fourrage." On a cru que ce mot venait de l'arabe "Al-chelfa" qui veut dire "Ce qui pousse après quelque chose autre." On l'applique généralement aux plantes qui poussent après que la végétation du printemps a disparu. Ce dernier mot indique l'aptitude de la plante à pousser pendant les chaleurs de l'été et se rapporte peut-être à sa faculté de produire plusieurs coupes pendant la même saison. Cependant, la première dérivation est probablement la bonne, l'espagnol "alfalfa" ayant été trouvé identique au mot arabe "Alfacfacah" au quinzième siècle par Fray Pedro de Alcala, un spécialiste distingué en langue arabe.

En Europe cette plante est toujours appelée luzerne. L'origine de ce mot est douteuse, il ne se rapporte nullement à l'Etat qui porte ce nom en Suisse car il était employé avant que la plante fut connu en Suisse. Il est peu probable qu'il soit dérivé de la vallée de Lucerna dans le nord de l'Italie comme le supposent généralement certains auteurs américains. Le vieux nom espagnol de la plante est "Userdas" qui peut être identique au mot "Louzerdo" employé dans le sud de la France. Il est plus probable que le mot luzerne vient de "lucerno" un vieux mot provençal.

Variétés: Il existe dans le commerce un grand nombre de variétés de luzerne dont quelques-unes sont de vraies variétés botaniques, c'est-d̀-dire qu'elles se distinguent des autres par des caractéristiques botaniques fixes. Par exemple, la luzerne du Turkestan a des folioles 
courtes, rondes et des graines ternes, d'autres ne sont que des variétés géographiques, leur nom signifie simplement que la graine a été produite dans un certain pays. D'autres encore font nettement preuve de qualités pratiques, telles que la rusticité, la résistance à la sécheresse et aux maladies, l'aptitude à taller, à produire de la graine, etc. Pour le Canada il n'y a que les variétés rustiques qui soient importantes. Par exemple, la luzerne arabe ou du Pérou est détruite par l'hiver, et généralement toutes les variétés qui viennent du sud, souffrent. Le cultivateur qui achète de la semence fera donc bien de s'assurer que la variété qu'on lui offre est adaptée au climat. Il est toujours bon de choisir une variété provenant d'un pays jouissant d'un climat semblable à celui où l'on se propose de cultiver la plante.

Culture: Le développement de la luzerne dépend en grande partie du sol. On peut la cultiver sur bien des sortes de sol, depuis les terrains sableux,sablo argileux jusqu'aux argiles lourdes. C'est dans les terres franches, profondes, à sous-sol ouvert et poreux, où rien ne gêne le développement des racines pivotantes, qu'elle vient le mieux. Comme la racine pénètre à une profondeur considérable la qualité du sous-sol est un facteur très important. Un sous-sol compact et impénétrable constitue un obstacle sérieux à la végétation de la luzerne. Pour la même raison, il est bien difficile d'obtenir une bonne levée sur sol plat, à sous-sol de roc, à moins que les racines puissent s'introduire au travers des fentes du roc. La luzerne résiste à une certaine quantité d'alcali dans le sol, mais il vaut mieux faire disparaître l'alcali de la surface avant de semer la graine et le tenir à cinq ou six pieds au-dessous de la surface par l'irrigation. L'acidité exerce toujours un mauvais effet. Dans les sols acides une application de chaux est avantageuse.

Climat: Comme les racines de la luzerne descendent à une grande profondeur la luzerne, quoique arrêtée dans sa croissance, n'est pas gravement affectée par une forte sécheresse. Elle aime une quantité raisonnable d'humidité mais elle redoute l'excès. Si le sous-sol est imperméable et que l'eau de surface ne puisse s'égoutter rapidement après une forte pluie, l'accumulation d'eau qui en résultera sera désastreuse ou du moins réduira la vitalité des plantes. Il faut donc tenir le sol bien égoutté surtout au commencement du printemps. Dans les champs mal égouttés la luzerne souffre; elle meurt parfois dans les fonds ou l'eau s'est accumulée. Tout excès d'eau dans le sol retarde la végétation des plantes et les empêche de se mettre à pousser de bonne heure. Dans un sol mal égoutté,les gels et dégels 
alternatifs font plus de mal que dans une terre bien drainée car le soulèvement du sol abime les racines. Souvent ce soulèvement est tel que la racine pivotante se casse et que la plante meurt.

Inoculation: De même que chez les autres plantes légumineuses, le développement de la luzerne est provoqué par des bactéries qui se logent dans des tubercules sur ses racines. Ces bactéries sont proches parentes de celles du trèfle d'odeur; il peut même se faire qu'elles soient les mêmes; quoi qu'il en soit, la luzerne vient bien dans les sols où le trèfle d'odeur a été cultivé.

Habitudes: On sème généralement la luzerne au printemps, les jeunes plantes sont délicates et redoutent la concurrence. On devrait donc s'attacher à enlever toutes les mauvaises herbes. Comme les plantes sont assez tendres la première année il vaut mieux leur donner toutes les occasions de devenir aussi fortes que possible afin qu'elles puissent résister à l'hiver. Il n'est pas à recommander de faucher la luzerne ou de la faire pâturer la première saison. Pendant la deuxième année ou les années suivantes la végétation parait de bonne heure et se maintient jusqu'à une époque avancée de l'automne, de nouveaux rejetons naissant du collet de la racine. Dáns des conditions favorables la luzerne dure très longtemps et produit abondamment.

Valeur agricole: La valeur nutritive de la luzerne était connue en Perse avant l'ère chrétienne. Elle était très estimée des Arabes. Aucune plante fourragère à l'heure actuelle ne l'égale au point de vue de la valeur nutritive et de l'utilité générale. Tous les bestiaux en raffolent, chevaux, moutons, bovins et porcs. Elle est même plus nourrissante que le trèfle rouge car elle renfeı me plus de protéine. C'est dans l'alimentation des vaches laitières qu'elle est le plus utile, mais elle est également précieuse dans l'engraissement de tous les animaux de la ferme et surtout des moutons et des porcs.

Fourrage: Les cultivateurs disent parfois que la luzerne ne fait pas de bon foin; c'est tout simplement parce qu'on ne l'a pas coupée au bon moment. Sa valeur comme plante à foin dépend de sa qualité nutritive et de son aptitude à produire un certain nombre de récoltes pendant la saison. De même que chez la plupart des plantes fourragères, la qualité du fourrage se détériore rapidement après que la plante a commencé à fleurir. Les tiges perdent leur succulence, deviennent très ligneuses, et les feuilles tombent facilement. 
Lorsque la plante a formé ses fleurs, de nouvelles tiges secondaires naissent des bourgeons situés au collet. C'est cette végétation secondaire qui doit former la deuxième coupe; par conséquent, la première coupe devrait être faite avant que ces rejetons se soient suffisamment développés pour être attrapés par la faucheuse. Il est donc bon de couper un peu tôt, même au risque d'avoir un peu moins de nourriture et un peu moins de foin. En fauchant au commencement de la période de floraison la première récolte sera un peu moins forte mais la deuxième se développera plus vite et sera plus considérable. Le fauchage hâtif donne donc, au total, plus de foin et du foin plus nutritif que le fauchage tardif. Lorsque la saison est longue et la température favorable on peut obtenir jusqu'à cinq ou six coupes par an. Dans les pays du nord, comme le Canada, on peut compter sur deux ou trois coupes. Dans les districts irrigués ou dans les endroits où la fenaison se fait par un temps sec, il n'est pas difficile d'obtenir un foin de luzerne vert clair et d'excellente qualité. Mais lorsque les pluies sont fréquentes ou les rosées abondantes après le fauchage, le foin est sujet à devenir brun ou jaune. Il perd beaucoup de sa valeur nutritive et de sa succulence. On fane généralement de la même manière que pour le trèfle rouge. Cependant la luzerne demande à être traitée avec plus de soin, car les feuilles tombent facilement et leur perte diminue beaucoup la quantité de fourrage.

Pâturage: La luzerne est peu cultivée en pâturage au Canada. L'essentiel, quand on désire obtenir un pâturage de cette plante, est de laisser les plantes bien prendre racine avant d'y mettre les animaux. Il n'est jamais bon de faire pâturer la luzerne avant la troisième année de croissance. Même dans les vieilles luzernières, des soins sont nécessaires si l'on veut empêcher que la plante ne meure par endroits. La luzerne n'a qu'une seule racine pivotante dont le collet fait généralement saillie un peu au-dessus de la terre. Elle est donc facilement abîmée par le piétinement, surtout lorsque le sol est ramolli par de fortes pluies. Comme les nouvelles tiges naissent de la couronne, la luzerne peut beaucoup souffrir du pâturage fait par les moutons. Il n'est pas à recommander de la faire paître tard en automne car le collet de la plante serait exposé et pourrait être détruit par l'hiver.

De même que ceux de trèfle rouge, les pâturages de luzerne peuvent causer la météorisation surtout chez les bovins et les moutons, si les animaux ne s'y habituent pas graduellement. Le danger est'surtout grand dans les journćes pluvieuses, lorsque les plantes sont humides de rosée. 
La luzerne donne un pâturage très estimé pour les porcs. Si le champ est divisé en deux ou trois parties pâturées à tour de rôle, la plante a le temps de reprendre et l'on peut nourrir un grand nombre de porcs sans courir le risque d'abîmer le champ.

Semis pour prairie ou pâturage: On peut semer la luzerne avec ou sans plante-abri selon les conditions de sol et de climat. Dans l'Ontario on emploie généralement l'orge comme plante-abri. Des essais effectués à la ferme expérimentale d'Indian Head, Sask., ont indiqué qu'il vaut mieux ne pas se servir de plante-abri dans les provinces des Prairies, afin que la luzerne puisse avoir à sa disposition toute l'humidité qui se trouve dans le sol. La quantité de graine à semeı dépend de sa qualité et de la nature du sol. Une quantité raisonnable est de vingt à vingt-cinq livres à l'acre. On obtient une récolte bien fournie en employant une plus petite quantité de graine, mais les semis épais produisent du foin et du pâturage de meilleure qualité.

Graine: Il se produit actuellement peu de graine de luzerne au Canada. Cette culture exige par dessus tout une température favorable pendant la floraison et la maturation. Si l'humidité est abondante, les organes végétatifs de la plante sedéveloppent fortement, la quantité de graine est insignifiante et celle-ci est mauvaise. On obtient la plus forte production lorsque le sol a juste assez d'humidité pour permettre à la graine de mûrir complètement. Les plantes exigent beaucoup de lumière et beaucoup de place, et, pour cette raison, il faut semer moins de graine que lorsqu'on veut avoir du foin et du pâturage; dix à douze livres de bonne graine donnent les meilleurs résultats. On peut employer, pour la production de la graine, n'importe laquelle des coupes de la saison mais il y a certaines objections à l'emploi de la première coupe dans ce but. Les insectes qui fécondent les fleurs sont moins nombreux à cette époque que plus tard, et la récolte de graine serait donc légère. De même, la floraison de la première récolte est relativement inégale et la qualité de la graine inférieure. Il y a une perte de foin lorsqu'on laisse la première récolte monter à graine car la luzerne qui a produit de la graine ne donne plus qu'une petite quantité de foin ou d'herbe. Pour ces raisons il est bon de couper la première récolte pour en faire du foin. Quant à savoir laquelle des autres récoltes devrait être utilisée pour la graine, cela dépend de la longueur de la saison et de la température. Au Canada c'est la deuxième coupe qui donne les meilleurs résultats.

Cultivée pour la graine, la luzerne est prête à être coupée lorsque la moitié des gousses sont brunes et que l'on peut facilement en 
faire sortir les graines en frottant. En la laissant trop se développer, on s'expose à perdre quelques-unes des graines les plus promptes à mûrir et par conséquent les plus précieuses.

On peut récolter de la niême façon que pour la graine de trèfle rouge mais il faut manier la récolte avec le plus grand soin pour éviter la perte de graine. Pour le battage on peut employer une batteuse ordinaire mais une batteuse à trèfle est préférable.

Qualité de la graine: Les graines sont réniformes et d'un brun jaunâtre, ̀̀ peu près deux fois plus longues que larges. Dans la luzerne ordinaire leur surface est luisante; dans la variété Turkestan à cause du revêtement de substance cireuse que l'on peut aisément faire disparaittre en frottant, la surface est terne. Le poids régulier est de soixante livres au boisseau.

Impuretés: Les jeunes plantes de luzerne sont très délicates et facilement étouffées par les mauvaises herbes. On détruit ces mauvaises herbes et on fortifie la jeune luzerne en passant la faucheuse sur le champ plusieurs fois la première saison. Dans les vieilles luzernières les plantes, quoique généralement très vigoureuses, sont parfois étouffées en certains endroits par des herbes agressives. Pour éviter ces accidents il faut choisir de la graine aussi propre que possible. Les graines de mauvaises herbes les plus communes dans la luzerne de commerce sont la sétaire verte, le plantain, l'herbe à poux, le chougras, la chicorée, la sétaire jaune et la persicaire. D'autres mauvaises herbes dangereuses, mais dont les graines sont moins communes, sont la bardane, la moutarde, le silène noctiflore, le silène enflé, la cameline et le chardon du Canada.

Maladies: La luzerne est moins sujette aux maladies que le trèfle rouge. La cuscute est son pire ennemi. La cuscute de la luzerne, qui est généralement appelée Cuscuta Epithymum Murr., est un parasite jaunâtre, sans feuilles, composé d'une masse de fils ténus d'où naissent de nombreuses racines appelées suçoirs. Ces suçoirs pénètrent dans les tiges de la luzerne où ils absorbent la nourriture prête pour l'usage de la plante-hôte. Les fleurs sont blanches et serrées, en grappes rondes. La cuscute paraît d'abord en plaques insignifiantes, éparpillées sur toute l'étendue du champ, mais ces plaques augmentent graduellement et infestent tellement la récolte qu'au bout de quelques années celle-ci ne produit plus rien. Le meilleur moyen d'éviter ce fléau est d'acheter de la graine qui soit absolument propre. Mais si la cuscute s'était établie dans une luzer- 
nière, il faudrait détruire immédiatement les plantes infestées. Le fauchage ne fait qu'enlever la cuscute sur les parties supérieures de la graine et n'affecte pas celle qui se trouve sur le collet ou près du collet, où elle vit pendant l'hiver.

C'est là peut être l'explication de la propagation désastreuse de la cuscute. Il est également possible que ces parties, qui n'ont pas été enlevées par la faux peuvent former des graines qui donnent naissance à de nouvelles plantes l'année suivante. On ne sait pas encore exactement si c'est de cette fason ou de l'autre que la cuscute du trèfle se répand au Canada.

\section{LUZERNE FAUGILLE (Medicago falcata L.).}

Nom anglais: Yellow Lucerne.

Description botanique: La luzerne faucille est proche parente de la luzerne cultivée. C'est une plante très vivace, à racine pivotante, profonde, et à nombreuses tiges. Celles-ci sont très différentes de celles de la luzerne ordinaire. Elles sont rarement tout à fait dressées mais ascendantes et souvent même couchées à la base. Elles sont plus grêles que les tiges de la luzerne, plus ligneuses, surtout à la base. Les feuilles sont semblables mais généralement ont des folioles plus étroites. Les fleurs un peu plus petites que celles de luzerne, et jaune clair, sont en grappe, comme dans la luzerne, mais cette grappe est généralement plus courte et renferme un plus petit nombre de fleurs. Le fruit n'est pas tordu comme celui de la luzerne mais seulement un peu courbé, comme une faucille.

Distribution géographique: La luzerne faucille est propre à l'ancien monde où elle est assez répandue. Elle se rencontre en Angleterre, dans l'ouest et le centre de l'Europe, dans l'ouest et le centre de la Scandinavie, la Russie et dans presque toute la région au nord de l'Himalaya.

Habitat et culture: Elle se rencontre généralement dans les sols pauvres, sableux ou graveleux; elle résiste mieux que la luzerne ordinaire à la sécheresse et aux grands froids. Elle convient donc mieux que celle-ci pour les climats rigoureux et les sols pauvres.

Valeur agricole: Elle n'aura jamais l'importance de la luzerne ordinaire à cause de ses tiges couchées et même rampantes et de ses 
rendements relativement faibles. Règle générale, elle produit peu de graine et la petite quantité de celles qui se forment est encore bien réduite au cours des manutentions.

La luzerne faucille se compose d'un grand nombre de types différents, à végétation très diverse, et par conséquent de valeur agricole variable. Tous, cependant, offrent, à des degrés variables, les mêmes inconvénients et aucun d'eux, autant que l'on sache à l'heure actuelle, ne peut faire concurrence à la luzerne ordinaire. Cependant, la luzerne faucille joue un rôle important en agriculture, comme on pourra s'en rendre compte en lisant la description ci-jointe de la luzerne rustique ou moyenne.

LUZERNE RUSTIQUE (Medicago falcata L. $\mathrm{x}$ sativa L.).

Autres noms latins: Medicago media Pers., M. silvestris Fr.

Autre nom français: Luzerne moyenne.

Noms anglais: Variegated alfalfa, Sand lucerne.

Description botanique: La luzerne faucille, comme nous disions plus haut, est proche parente de la luzerne commune; de fait les deux luzernes ont été prises pour une seule espèce par certains auteurs, surtout à cause des types intermédiaires qui existent entre les deux et qui rendent la distinction difficile ou même impossible. On peut comprendre tous ces types sous le même nom: luzerne rustique ou moyenne. Toutefois il n'y a pas à douter que la luzerne faucille et la luzerne commune sont deux espèces distinctes et qui peuvent être facilement distinguées l'une de l'autre par la couleur de leurs feuilles et la forme de leurs fruits. La luzerne rustique ou luzerne moyenne, qui paraît faire exception, n'est pas une variété de l'unè ou de l'autre, mais un croisement des deux espèces, comme le mulet est un produit du croisement du cheval et de l'âne. 
A cause de son origine hybride la luzerne rustique ressemble, sous certains rapports, à la luzerne ordinaire, et sous d'autres, à la luzerne faucille. On la distingue généralement par ses fleurs. Comme c'est un croisement entre une espèce jaune et une espèce pourpre ses fleurs sont un mélange de jaune et de pourpre. Ce mélange a pour résultat une couleur jaunâtre sale, singulière caractéristique générale du premier hybride, c'est-à-dire du produit obtenu en croisant la luzerne commune pure avec la luzerne faucille pure. Toutefois, dans un champ de luzerne rustique on trouve des fleurs de toutes les couleurs, de jaune à pourpre verdâtre foncé. La couleur dépend, jusqu'à un certain point, de la phase du développement: la même fleur change généralement de couleur avec l'âge c'est-à-dire que toutes les nuances peuvent être présentes dans une même plante mais la cause principale de la variation est la manière dont les fleurs sont fécondées. La luzerne rustique est différente de la plupart des autres plantes hybrides en ce qu'elle est fertile, c'est-à-dire qu'elle peut produire une abondance de graine de bonne qualité. Les fleurs d'une plante du premier hybride peuvent être fécondées par les fleurs d'un autre hybride primaire, ou encore par les fleurs de la luzerne pure, s'il s'en trouve dans le voisinage. Dans chaque cas le résultat est un mélange ou une nouvelle combinaison des couleurs originales.

Habitat: La luzerne rustique se rencontre naturellement dans les endroits où la luzerne commune et la luzerne faucille poussent ensemble.

Culture: Elle n'a de valeur agricole que dans les régions où le climat est trop rigoureux, où le sol est trop pauvre pour la luzerne commune, car elle hérite d'une partie de la rusticité de la luzerne faucille. Le nom "Luzerne des sables" sous lequel elle est connue en Europe, indique qu'elle est adaptée aux sols pauvres et secs.

Climat: Elle doit sa réputation au fait qu'elle résiste mieux aux grands froids que la luzerne commune, ce qui la rend spécialement intéressante au Canada.

Valeur agricole: Pour la production du fourrage l'hybride primaire n'a pas autant de valeur que la luzerne commune, la production est plus faible, la qualité nutritive n'est pas aussi bonne, le mode de végétation couchée qu'elle hérite de la luzerne faucille affecte le rendement et la qualité, le danger de verse est plus grand qu'avec la 
luzerne commune, surtout lorsque la végétation est épaisse. Enfin sa végétation rampante la rend plus difficile à couper, la faucheuse ne pouvant atteindre au-dessous des tiges couchées.

Variétés: La luzerne rustique varie énormément à cause de son origine hybride. Il existe bien des "variétés" commerciales, d'une valeur agricole assez différente. La plus fameuse, et, à l'heure actuelle, la plus importante, incontestablement, est la luzerne Grimm qui est rustique pour les districts du Canada où l'on cultive la luzerne, et le nord des Etats-Unis. De même, la luzerne canadienne rustique, qui, d'après les essais faits par le professeur C. A. Zavitz au collège d'agriculture de Guelph, Ontario, est égale à la luzerne Grimm et beaucoup plus rustique que la variété ordinaire, offre un intérêt spécial pour le Canada.

La luzerne Grimm et la luzerne rustique canadienne, de même que toutes les autres variétés de luzerne rustique, ne sont nullement uniformes mais comprennent des plantes de valeur très différente. Les unes ressemblent à la luzerne ordinaire au point de vue de la végétation et du rendement, les autres sont comme la luzerne faucille. A cause de cette variation il est très possible d'obtenir par la sélection des variétés très productives qui joignent aux qualités désirables de la vraie luzerne la rusticité de la luzerne faucille.

La luzerne est la merveille du mesnage des champs.-Olivier de Serres, Thêâtre d'agriculture eı mesnage des champs, 1600.

Les qualités alimentaires de la luzerne diminuent à mesure qu'elle s'éloigne du midi; mais malgré cela aucun fourrage ne peut lui être comparé pour la qualité, aucun n'entretient les animaux dans une aussi bonne graisse, n'augmente autant l'abondance-de lait dans les vaches et autres femelles qui nourrissent.-Abbé Rozier, Cours d'A griculture, 1734-1793.

Bonhomme, me disait-ii, sang bétail on ne fait rien qui vaille, on n'a ni grain, ni foin, ni paille. Encore faut-il savoir nourrir, rester à la grange et ne pas courir. Je dis partout; petite brassée et souvent, on arrive au bout de i'an; - et le bon nourrisseur vaut le bon laboureur.-Souviens-toi de ca, mon petit.-Bujault.

J'en vins à considérer les actions admirables que la Providence fait accompiir a la nature, et entr'autres choses, mes yeux se fixalent sur les branches des vignes, des pois et des courges qui paraissaient avoir quelque sentiment de la faiblesse de leur nature; incapables de se soutenir ellesmémes, ces plantes émettent de petits bras, comme des fils, qui, dès qu'ils rencontrent une petite branche ou un rameau, s'y attachent pour ne plus s'en séparer. Bernard Palissy. Jardin délectable. I 508-89. 



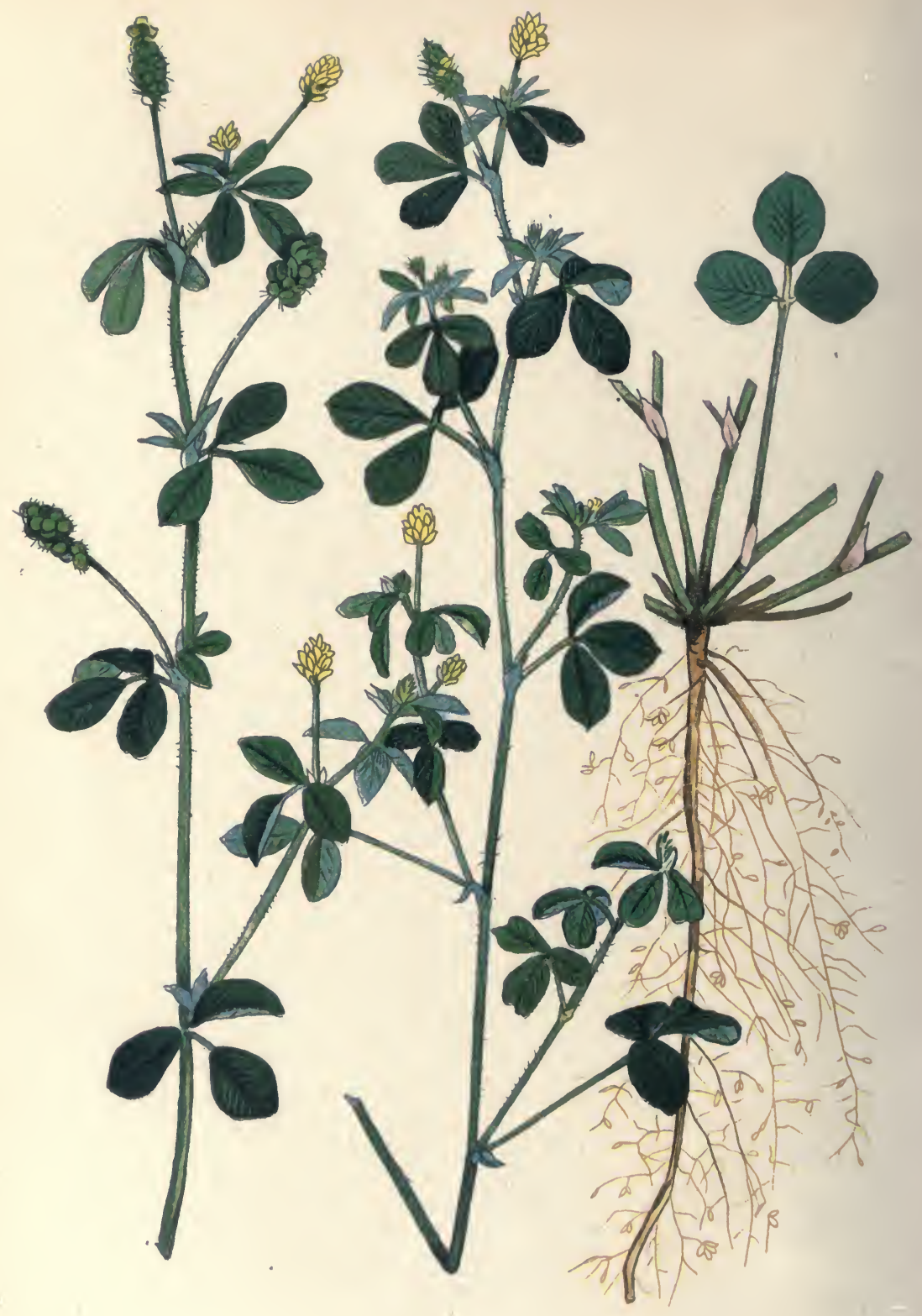

Planche 22 


\section{MINETTE ou LUPULINE (Medicago lupulina L.)}

Planche 22 ; graine, planche 27 , fig. 35 .

Autres noms français: Mignonette, trèfle jaune (à cause de ses fleurs), trèfle noir (à cause de ses fruits), Bujoline, luzerne houblonnée.

Noms anglais: Yellow Trefoil, Black Medick.

Description botanique: La minette ou lupuline est proche parente des luzernes mais elle dure moins longtemps. Quoique bisannuelle elle ne reste parfois qu'une seule année sur le terrain. Elle a une racine pivotante qui s'enfonce généralement à une profondeur d'un pied et ne porte que peu de branches. Les tiges, qui naissent en grand nombre du collet de la racine, sont couchées à la base ou ascendantes. Elles rampent souvent à la surface du sol mais ne produisent pas de racines secondaires. Les plantes sont donc étalées, d'autant plus que les tiges sont généralement très rameuses. Les feuilles se composent de trois folioles dont chacune a une courte queue. Les fleurs sont jaunes, beaucoup plus petites que celles des autres espèces de Medicago mentionnées, et en capitules courts et cylindriques. La minette ressemble beaucoup à certaines espèces de trèfle à fleurs jaunes (Trifolium), mais il est facile de l'en distinguer. Dans les vrais trèfles les fleurs ne tombent pas lorsque la floraison est terminée, elles se forment et restent sur la plante, cachant les gousses et brunissant les capitules, jusqu'à ce que le fruit soit mûr. Dans la minette au contraire, les fleurs tombent dès que la floraison est terminée et les gousses sont donc visibles en mûrissant. Ces gousses sont petites, réniformes, noires, à angles rudes, d'où le nom anglais de "Black Medick". Elle tombent sans se rompre. Il n'y a qu'une graine par gousse.

Distribution géographique: La minette est propre à toute l'Europe, sauf aux parties les plus reculées vers le nord, au nord de l'Afrique et à l'Ouest de l'Asie. Elle n'est pas indigène à l'Amérique du nord mais est assez répandue sur toute l'étendue du continent où elle croît généralement comme une mauvaise herbe.

Historique: On la cultive en Angleterre depuis environ cent cinquante ans. Dans l'Europe centrale ses qualités fourragères sont appréciées depuis le commencement du dix-neuvième siècle. Elle est peu cultivée au Canada. On la trouve généralement dans le trèfle 28550-16 
rouge et le trèfle d'alsike ou elle est une impureté. On doit alors la considérer comme une mauvaise herbe car elle est trop mûre lorsque les trèfles sont prêts à être fauchés.

Culture: A cause de ses racines peu profondes la minette est moins difficile sur le choix du sous-sol que les luzernes et la plupart des trèfles. Elle vient relativement bien sur un terrain assez pauvre mais réussit mieux sur ceux qui ne sont ni trop fermes ni trop frais. La chaux est nécessaire à son développement et une quantité généreuse de potasse et d'acide phosphorique est avantageuse.

Climat: Quoique ses racines ne soient pas très profondes, la minette endure une certaine somme de sécheresse sans en souffrir beaucoup. Elle résiste modérément au froid. Elle préfère un climat tempéré et donne une végétation superbe lorsque l'humidité est abondante dans l'air et dans le sol.

Valeur agricole: La minette est surtout désignée pour les rotations courtes à cause de son caractère bisannuel ou même annuel. Ses tiges semi-couchées, sa végétation étalée, la rendent peu avantageuse pour le foin, car la plupart de la plante échappe à la faux. C'est en pâturage qu'elle est plus utile. Elle se met à pousser plus tôt que la plupart des herbes de pacage, se développe rapidement et produit un fourrage vert, très précieux. Elle résiste remarquablement bien au pâturage au ras du sol, et, pour cette raison, convient tout particulièrement aux moutons.

Sa valeur nutritive et sa productivité étant inférieures à celles de la luzerne ou du trèfle rouge, on doit lui préférer ces dernières plantes partout où elles peuvent être cultivées. On ne doit pas l'employer seule dans les fourrages et seulement en quantité limitée dans les mélanges. Une proportion trop forte de cette plante pourrait nuire à la végétation des autres herbes. Malgré son peu de durée, elle produit beaucoup de graine et finirait par étouffer les autres plantes de pâturage. Vingt livres de bonne graine à l'acre suffisent.

Graine: La graine de la minette est relativement bon marché; on l'emploie parfois pour falsifier le trèfle rouge. On la trouve souvent dans les échantillons commerciaux de trèfle rouge, de trèfle d'alsike ou de luzerne commune. Elle a la même couleur que celle de la luzerne avec laquelle on la confond parfois. Les graines de la minẹtte sont plus épaisses, plus courtes et ovoïdes, tandis que celles de la luzerne sont réniformes ou parfois irrégulièrement anguleuses. La graine pèse soixante livres au boisseau. 
ANTHYLLIDE VULNÉRAIRE (Anthyllis Vulneraria L.).

Graine, planche 27, fig. 36 .

Autres noms français: Trèfle jaune des sables, Vulnéraire.

Nom anglais: Kidney Vetch.

Description botanique: L'anthyllide est une plante vivace à souche courte, d'où naissent de nombreuses tiges aériennes de six pouces à un pied de hauteur. Les feuilles sont nombreuses, elles se composent d'un certain nombre de folioles en paires et d'une foliole séparée, beaucoup plus grosse que les autres. Les fleurs sont en capitules serrés, généralement en paires. Elles sont jaunes ordinairement, mais parfois blanches ou rouges. C'est une plante mellifère, souvent visitée par des insectes qui transportent le pollen d'une fleur à l'autre. Mais lorsqu'on isole les plantes de façon à empêcher les insectes de les visiter, les fleurs se fécondent automatiquement par leur propre pollen.

Distribution géographique: Elle est propre à toute l'Europe, sauf aux parties les plus septentrionales, au sud-ouest de l'Asie et au nord de l'Afrique.

Habitat: Elle croît spontanément dans les pacages secs, le long des chemins et des sentiers, sur les collines et les montagnes et dans les bois ouverts.

Culture: L'anthyllide vulnéraire donne une bonne récolte sur les sols sableux ou graveleux, trop pauvres pour la plupart des plantes légumineuses. Elle atteint son plein développement sur les terres riches en chaux. Elle supporte les grandes sécheresses sans souffrir et les gels et les dégels alternatifs l'affectent peu.

Valeur agricole: Dans certaines parties de l'Europe où la terre est trop pauvre pour le trèfle rouge, l'anthyllide est très utile. On s'en sert comme plante à foin ou à fourrage. Elle est appréciée du bétail. Elle produit assez peu cependant. On ignore si elle est adaptée au Canada. 


\section{SAINFOIN (Onobrychis sativa Lam.).}

Planche 23; graine, planche 27, fig. 37 .

Autre nom français: Esparcette commune.

Noms anglais: Sainfoin, Esparsette.

Description botanique: Le sainfoin est une plante vivace, à racine pivotante vigoureuse, qui devient assez ligneuse et très ramifiéc. Les tiges sont nombreuses, dressées ou ascendantes, et atteignent une hauteur de un à deux pieds. Les feuilles sont composées et consistent généralement en un grand nombre de folioles lesquelles, à l'exception de la foliole terminale qui est seule, se trouvent en paires le long de la nervure médiane. L'inflorescence riche, en forme d'épi, s'allonge et se dégarnit graduellement. Les fleurs, très voyantes, sont roses, à veines plus foncées. Elles sont riches en miel et permettent aux abeilles ordinaires et à d'autres insectes d'atteindre le fond du tube où le nectar est enmagasiné.

Distribution géographique: Le sainfoin est propre aux régions tempérées de l'Europe, de la Baltique au nord jusqu'à la Méditerrannée au sud, et de l'Atlantique à l'ouest jusqu'à la frontière de l'Asie à l'est. Il est également propre au sud de l'Asie.

Historique: On cultıve le sainfoin en France depuis plus de quatre cents ans et sa culture s'est répandue à tous les autres pays européens particulièrement l'Italie, l'Angleterre, la Suisse, et l'Allemagne, où on le considère également comme une plante fourragère importante. Il a été introduit aux Etats-Unis il y a près de quatrevingt-dix ans. Les essais de culture faits en ces quinze dernières années à la ferme expérimentale centrale d'Ottawa, permettent d'en recommander l'adoption dans certaines parties de l'est du Canada.

Culture: Dans l'Europe centrale, le sainfoin croît spontanément sur les côtes sèches et ensoleillées, sur les bords des bois, etc, partout où le sol est riche en chaux. La chaux est nécessaire à son développement et il vient bien à peu près partout où se trouve une quantité suffisante de cet élément. Il devrait réussir dans bien des districts à base de chaux de l'est du Canada. Sa végétation est excellente sur les terres franches, bien égouttées, profondes, poreuses et contenant une bonne proportion de chaux. Les argiles lourdes lui conviennent 


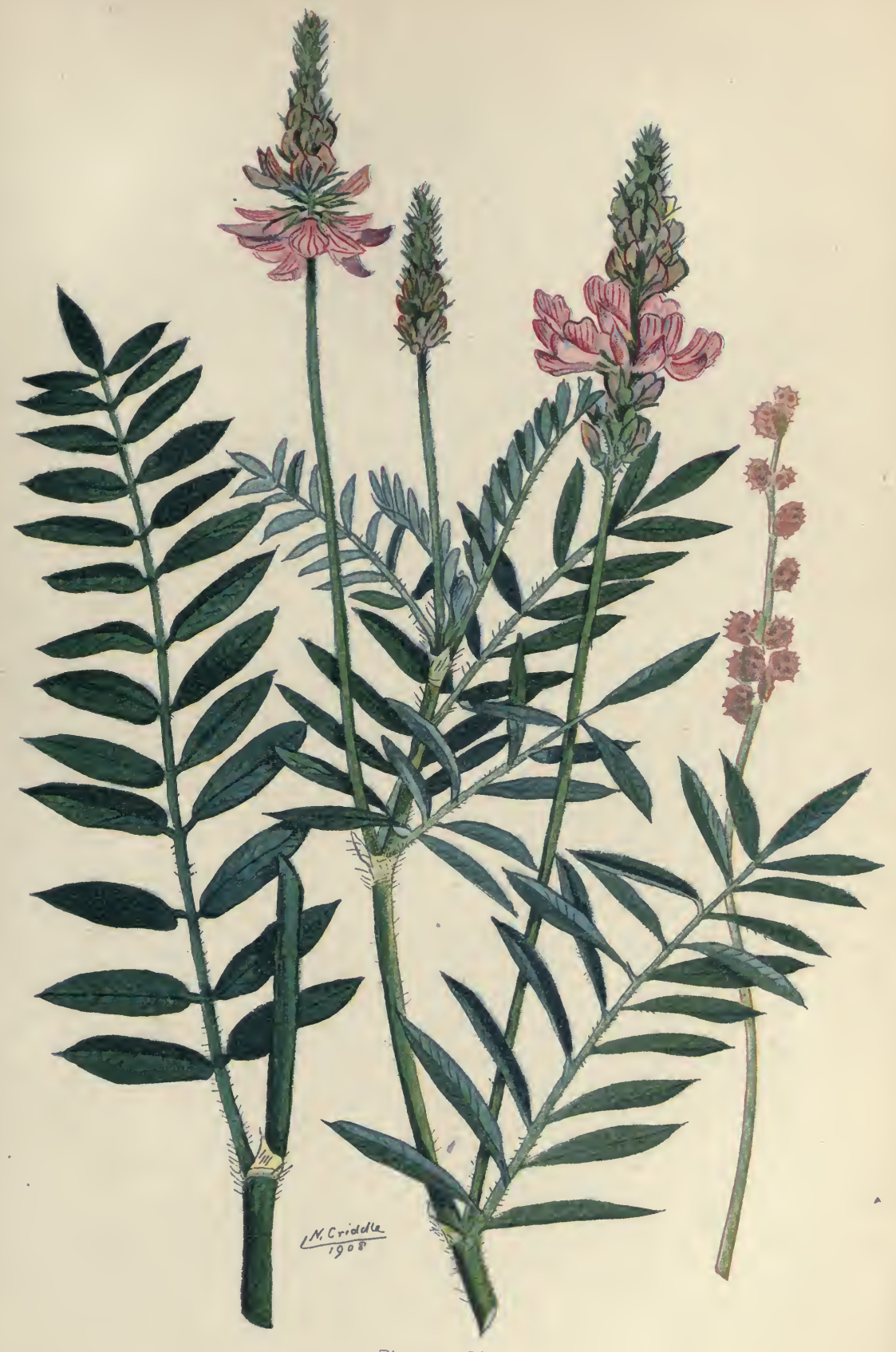

Planche 23

SAINFOIN

(Onobrychis sativa 

moins car sa végétation y est généralement trop lente et les terres basses, inondées pendant une partie de la saison, ne lui conviennent pas plus que les terres marécageuses ou celles dont le sous-sol est humide.

Climat: Le sainfoin est particulièrement adapté aux climats tempérés, à humidité moyenne. Ses racines profondes le rendent très résistant à la sécheresse et il donne une récolte étonnante là où la plupart des autres plantes fourragères souffriraient du manque d'humidité. Une fois établi, il résiste assez bien aux hivers de la vallée de l'Ottawa.

Végétation: Il est parfois difficile d'obtenir une bonne levée de sainfoin. La graine est souvent pauvre et ne donne qu'un faible pourcentage de plantes fortes et vigoureuses. Les mauvaises herbes l'étouffent souvent quand il est jeune et il est essentiel que la terre soit tenue propre. Une fois qu'il a bien pris racine sur un sol convenable il produit de nombreuses récoltes. En Allemagne il n'est pas rare de voir des champs de vingt ans. Cependant les essais à la ferme expérimentale centrale d'Ottawa montrent qu'il n'y a pas d'avantage à le laisser plus de trois ans sur le sol.

Valeur agricole: Il mérite d'être comparé à la luzerne mais il a l'avantage sur celle-ci de faire une meilleure pousse sur terrain pauvre. Son nom, qui est d'origine française, s'écrivait autrefois "Sain foin," nom que l'on a parfois ortographié par erreur "Saint foin".

Fourrage: Quand on veut en faire du foin, le sainfoin doit être coupé quand il est en fleurs. Après la floraison les tiges deviennent coriaces et le foin moins bon. Parfois on peut en tirer deux récoltes la deuxième année. Après la dernière coupe les plantes devraient avoir le temps de produire une végétation raisonable pour se protéger en hiver.

Pâturage: Le sainfoin pousse de très bonne heure et peut être pâturé aussitôt que la luzerne. Il donne un excellent pâturage, dont les moutons sont particulièrement friands. Il ne cause pas la météorisation et convient donc mieux que la luzerne pour les vaches et les moutons. Cultivé comme plante à foin ou à pâturage, on doit mettre de trois à cinq boisseaux de graine à l'arre. 
Production de la graine: Les vieux champs de sainfoin qui donnent une récol te de foin relativement faible produisent la meilleure récol te de graine. La récolte est prête à être coupée lorsque les gousses sont d'un brun clair. Une coupe tardive entraîne des pertes considérables car les gousses tombent facilement, même avec les manutentions les plus soigneuses.

Qualité de la graine: La graine que l'on trouve dans le commerce est presque toujours non décortiquée, c'est-à-dire qu'elle est encore dans sa gousse. Les gousses sont presque semi-circulaires, un peu aplaties, d'environ un-huitième de pouce de long, et un peu moins large. Leur surface est couverte d'un filet-réseau, faisant saillie, qui est souvent armé d'épines. Le bord extérieur du demicercle est aplati en une bordure bien accusée, munie de dents fortes et pointues. Les gousses bien mûres sont d'un brun rougeâtre et ont un lustre métallique caractéristique, surtout quand elles ne sont pas trop vieilles. La graine enveloppée de sa gousse pèse environ vingt-six livres au boisseau. Les vraies graines sont réniformes et d'un brunolive à marron. Il n'y en a qu'une par gousse.

\section{VESCE COMMUNE (Vicia sativa L.)}

Planche 24; graine, planche 27, fig. 38 .

Noms anglais: Common Vetch, Tare, Spring Vetch.

Description botanique: La vesce commune est une plante annuelle, proche parente du pois. Les tiges, généralement rameuses près de la base, sont hautes de deux à trois pieds, anguleuses et plus ou moins velues. Les feuilles, nombreuses et composées, consistent en un certain nombre de folioles séparées, arrangées en paires le long d'une nervure centrale. A la partie supérieure il n'y a que la nervure centrale des folioles qui soit développée. Ces nervures se transforment en feuilles sensibles, appelées vrilles, qui s'entourent autour de tous les objets avec lesquels elles viennent en contact et aident ainsi à soutenir les tiges faibles de la plante. Les folioles sont oblongues, carrées aux extrémités, à pointe minuscule et étroite. Les fleurs sont en. paires à la base des feuilles. Elles sont sans queues ou ont des queues très courtes. Elles sont généralement de pourpre à rose et 


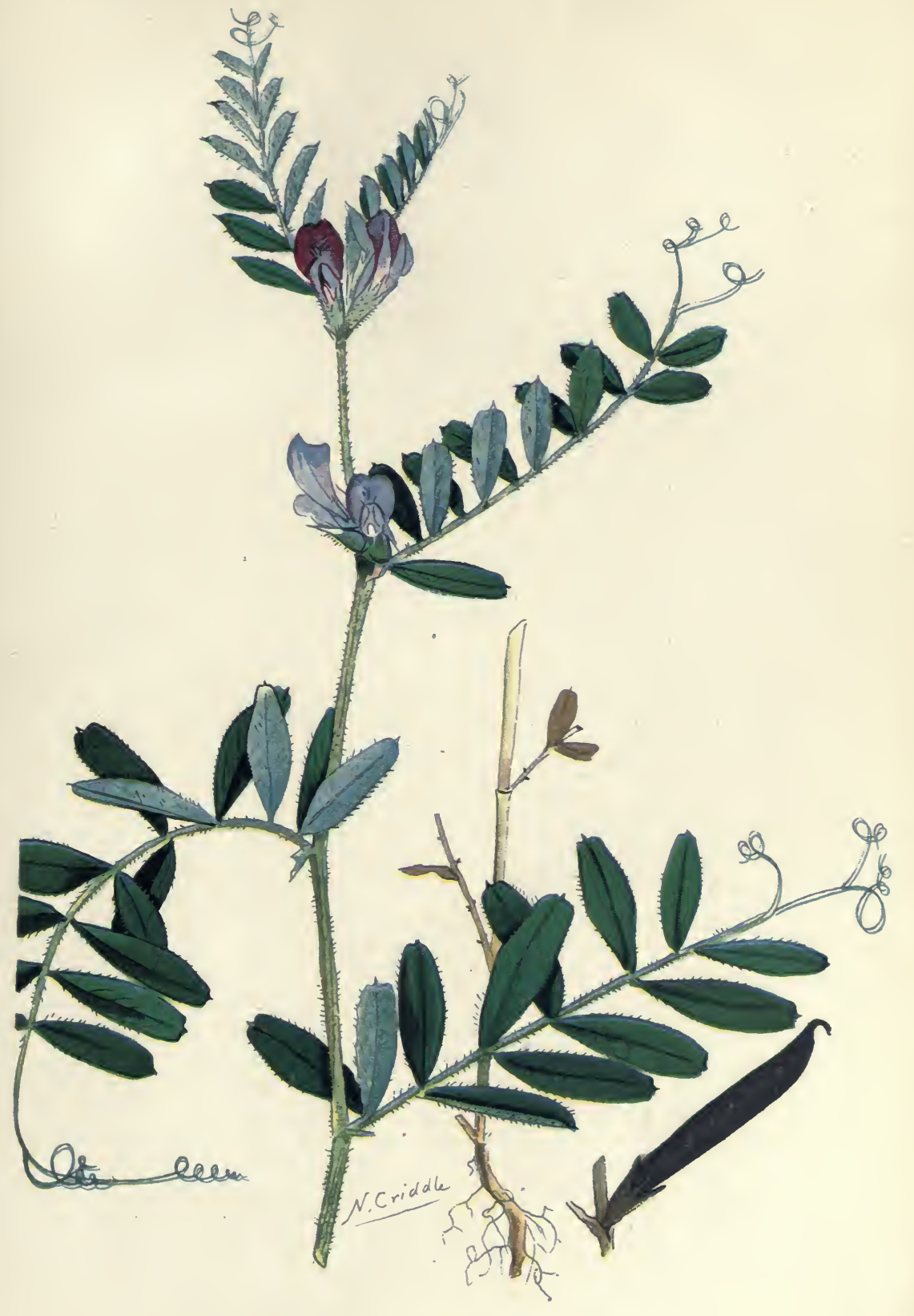

Planche 24

Vesce COMMUNe Cultivée (Vicia sativa L.) 

parfois entièrement blanches. La fécondation, comme celle des fleurs du pois, se fait sans l'aide des insectes. Le pistil d'une fleur est fécondé par le pollen de la même fleur. Il est rare que la fécondation soit croisée.

Distribution géographique: La vesce commune est propre à l'Europe où elle abonde, sauf dans les parties les plus septentrionales. Elle est répandue également dans certaines parties du nord de l'Afrique et du sud-ouest de l'Asie. Elle n'est pas indigène à l'Amérique du nord mais a été importée d'Europe. Les anciens Romains connaissaient sa valeur fourragère. Elle est aujourd'hui cultivée dans toute l'Europe. Au Canada sa culture est limitée principalement au sud-ouest de l'Ontario.

Végétation: Quoique les pois et les vesces soient proches parents leur développement est essentiellement différent. Dans le pois, la tige principale pousse pendant toute la durée de la plante mais les branches sont moins vigoureuses. Dans la vesce, la tige principale cesse bientôt de croître et de fortes branches latérales naissent de la basse. Sous ce rapport la vesce agit comme ces plantes qui se mettent à pousser tard dans la saison, se reposent pendant l'hiver et complètent leur développement l'année suivante. Ce sont des annuelles d'hiver et qui exigent une période de repos afin de se développer convenablement. Chez la vesce commune, que l'on sème au printemps à cause de son peu de rusticité, la période de repos est très courte. Toutefois, lorsque la première partie de l'été est fraîche, la plante peut rester dans un état stationaire pendant bien des semaines, ce qui prouve sa parenté aux annuelles d'hiver.

Variétés: Comme les pois la vesce commune comprend un grand nombre de variétés qui diffèrent l'une de l'autre au point de vue du développement, de l'époque de la floraison, de la couleur des fleurs et de la graine, etc.

Valeur agricole: Comme fourrage pour les vaches laitières, la vesce commune verte est très estimée pour sa valeur nutritive et son aptitude à augmenter la production du lait. On a longtemps cru qu'elle arrêtait la production du lait quand on la donnait mûre mais il a été prouvé par des essais récents qu'elle n'exerce aucun mauvais effet sur la quantité ou la qualité du lait produit.

Graine: Les graines sont rondes et aplaties, noires dans la plupart des variétés, mais grises, blanches, ou rougeâtres dans d'autres. 
VESCE VELUE (Vicia villosa Roth.)

Planche 25; graine, planche 27 , fig. 39.

Autre nom français: Vesce de Russie.

Noms anglais: Hairy Vetch, Winter Vetch.

Description botanique: Comme son nom l'indique cette plante est une annuelle d'hiver. (Voir glossaire). Elle atteint de deux à quatre pieds de hauteur, s'enroule et s'étend dans toutes les direciions comme la vesce commune, dont on la distingue facilement, même avant que les fleurs soient produites, par les poils qui la recouvrent. Toute la plante est en effet recouverte de poils longs, mous, étalés, qui lui donnent parfois un aspect blanc laineux. Les feuilles sont composées, comme celles de la vesce commune, mais les folioles s'amincissent graduellement vers l'extrémité et les vrilles sont plus branchues Les fleurs sont disposées en grappes riches, à longues queues, plus petites que celles de la vesce commune et de couleur pourpre à bleu pâle.

Distribution géographique: La vesce velue est propre à l'Europe et au sud-ouest de l'Asie. On a commencé à la cultiver en Europe vers le millieu du dix-neuvième siècle. C'est à peu près à cette époque qu'elle a été introduite dans l'Amérique du nord. On la cultive peu au Canada, et presque exclusivement dans les districts fruitiers de la province d'Ontario.

Culture: La vesce velue n'exige pas un sol aussi bon que la vesce commune. On peut cultiver sur les sols pauvres et sablonneux mais bien entendu elle produit plus sur les terres riches et bien préparées. Elle est beaucoup plus rustique que la vesce commune et supporte mieux les hivers du sud de l'Ontario.

Végétation: On la sème généralement en été, l'époque exacte des semis dépend du climat. Le but principal est d'obtenir une bonne levée avant l'arrivée des froids. Dans le sud de l'Ontario les plantes ne sont pas détruites par l'hiver et la pousse recommence au printemps.

Valeur agricole: Dans l'Ontario on la cultive presque exclusivement comme plante-abri et engrais vert, dans les vergers. Elle 


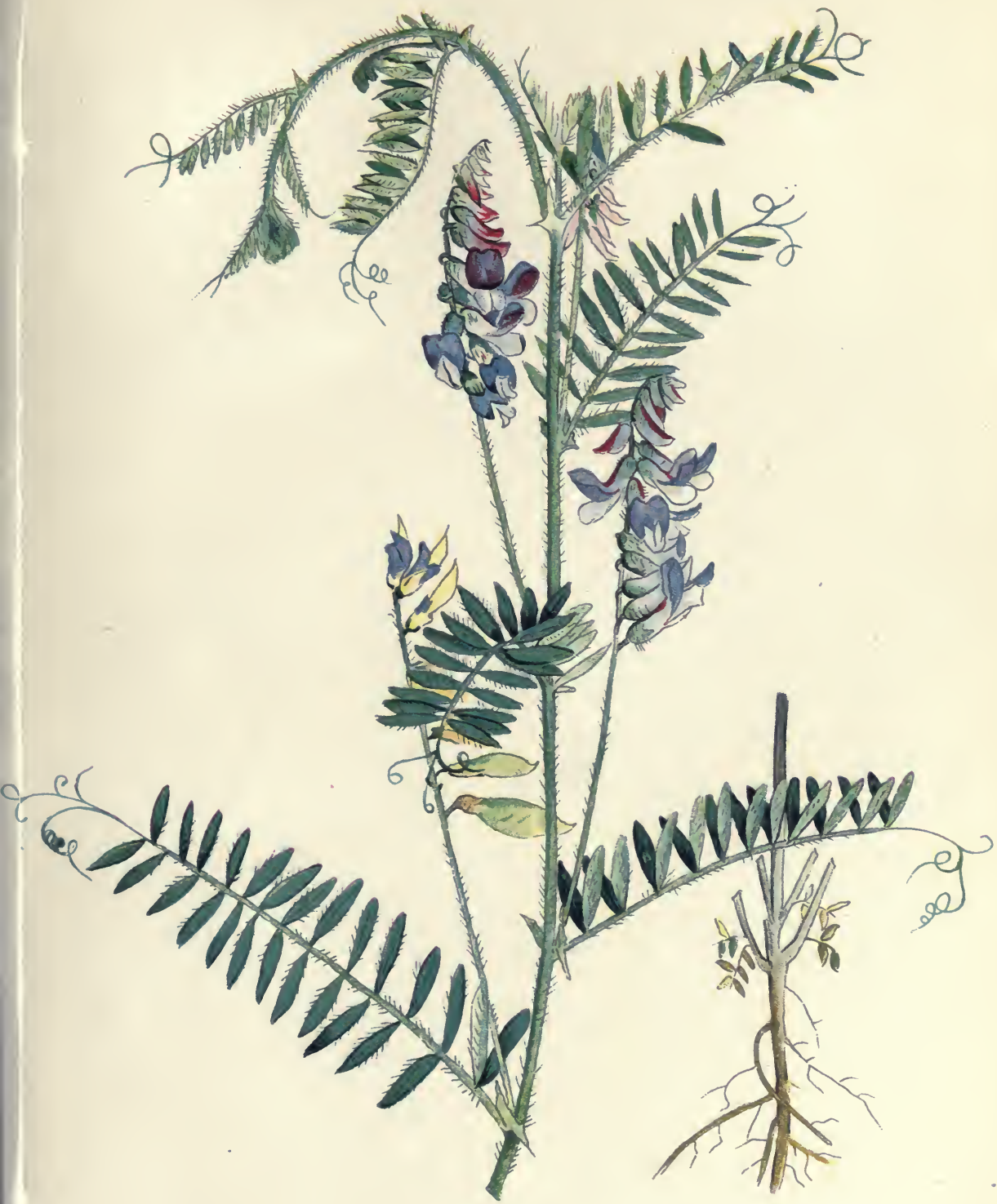

Planche 25

VESCE VELUE

(Vicia villosa Roth.) 

produit une grande quantité de matière végétale et souvent la pousse est trop forte pour qu'on puisse facilement l'enfouir à la charrue. Il suffit d'un demi-boisseau de bonne graine à l'acre pour obtenir une bonne levée. Cultivée pour sa graine, on la sème seule ou avec du seigle d'hiver qui soutient les tiges et retarde la fente des gousses mûres, qui réduit toujours beaucoup le rendement de semence. Le prix élevé de la graine dans le commerce est un obstacle à l'emploi de la vesce velue comme plante fourragère.

Graine: Les graines sont un peu plus petites que celles de la vesce commune, rondes, non aplaties, et leur couleur varie de brun foncé à noir grisâtre.

\section{FÈVE A CHEVAL (Faba vulgaris Moench.).}

Autre nom français: Féverole, fève commune.

Nom anglais: Horse Bean.

Description botanique: La fève à cheval est une plante annuelle qui atteint généralement une hauteur de deux à trois pieds. Elle pousse tout à fait droite. Elle ne s'enroule pas comme les fèves et ne grimpe pas comme les pois et les vesces. Les feuilles se composent d'une à trois paires de grosses et larges folioles. Elles n'ont pas de vrilles. Les fleurs sont portées en grappes de deux à cinq fleurs. Elles sont grosses et voyantes, blanches avec deux grosses taches noires ou d'un pourpre foncé. Les gousses, qui ont parfois jusqu'à cinq pouces de longueur, renferment cinq à six grosses semences séparées l'une de l'autre par un tissu mou et spongieux.

Historique: La fève à cheval est une ancienne plante en agriculture et son origine n'est pas connue. On la dit originaire de la Perse mais les preuves ne sont pas concluantes. On la cultivait dans l'Europe centrale des milliers d'années avant l'ère chrétienne et on en a trouvé de grandes quantités de graines dans les fouilles de Troie.

Sa culture est encore assez importante dans le sud et le centre de l'Europe, l'Angleterre et l'Egypte, mais elle est graduellement remplacée par d'autres légumineuses.

Variétés: Il existe un certain nombre de variétés qui se distinguent les unes des autres principalement par la dimension des graines. 
Valeur agricole: La fève à cheval était cultivée par les Grecs et les Romains et les graines étaient employées pour faire du pain, des gâteaux et du gruau. Dans ces parties de l'Europe où elle est l'objet d'une culture assez importante on l'emploie toujours pour l'alimentation de l'homme aussi bien que pour la production des fourrages. Au Canada elle est surtout utile comme culture intercalaire, ou "planteabri" dans les jeunes vergers où, semée en été, elle absorbe l'humidité du sol, arrête la végétation tardive du bois des arbres fruitiers et force la végétation du printemps à murir avant que les gelées soient à craindre. Elle enrichit le sol par l'azote qu'elle accumule et ses tiges, quoique détruites par les gelées d'automne, aident à retenir la neige.

Graine: Dans certaines variétés de la fève à cheval les graines ont près de trois pouces de long et un demi pouce de large. Elles sont plates avec une cicatrice profonde à une extrémité. Elles sont généralement d'un brun-rougeâtre.

\section{GESSE GULTIVÉE (Lathyrus sativus L.)}

Graine, planche 25, fig. 40 .

Autres noms français: Lentille d'Espagne, Lentille Suisse, Pois carré, Pois Breton, Pois gras, Pois de brebis.

Nom anglais: Grass pea.

Description botanique: La gesse cultivée est annuelle. Les tiges sont aplaties, plus grêles que celles du pois commun, et atteignent une hauteur de deux à trois pieds. Les feuilles se composent d'une à deux paires de folioles étroites, herbeuses et ont des vrilles rameuses. Les fleurs sont blanches et solitaires. Les gousses ont environ un pouce de long et un-demi pouce de large: elles sont plates et contiennent de trois à quatre graines. Ces dernières sont nettement anguleuses généralement jaune à vert jaunâtre.

Distribution géographique: La gesse cultivée est originaire du centre de l'Europe. On la cultive un peu dans le sud de l'Ontario.

Culture: Elle preffère une terre franche, pas trop lourde et se plait dans des terrains qui ne sont pas trop humides. 
Valeur agricole: Dans certains endroits elle est très employée comme plante fourragère car elle donne une nourriture saine qui n'a jamais fait de mal au bétail. Elle résiste à la bruche du pois et est donc précieuse lorsque le pois ordinaire ne peut être cultivé à cause de cet insecte.

\section{GESSE DES BOIS (Lathyrus silvestris L).}

Autre nom français: Gesse sauvage.

Nom anglais: Flat pea.

Description botanique: La gesse des bois est vivace, à racine vigoureuse, consistant en une souche forte qui émet de nombreuses branches latérales et des racines secondaires. Les tiges, qui atteignent une hauteur de deux à six pieds, sont semi-couchées ou ascendantes, grimpantes, et s'enroulent dans toutes les directions. Elles sont très rameuses, anguleuses, et munies de deux larges ailes. Chaque feuille se compose d'une paire de folioles et d'un groupe de vrilles au bout de la nervure médiane, allongée, de la feuille. Les fleurs sont voyantes, d'un rose foncé, et un peu plus petites que celles des pois ordinaires.

Distribution géographique: La gesse des bois est indigène à l'Europe. Elle croît spontanément dans les terrains boisés, sur les collines rocheuses, parmi les arbustes, sur les bords des bois, dans les fourrés, etc.

Culture: La gesse des bois n'exige pas un sol de qualité exceptionnelle; elle vient tout aussi bien sur les terrains pauvres. Ce sont les terrains sablo-argileux qui donnent les plus fortes récoltes mais on peut aussi obtenir de bons rendements sur les argiles lourdes et les sables. Etant donnée la profondeur qu'atteignent les racines, la nature du sous-sol est encore plus importante que celle de la surface du sol. Le bon égouttement du sous-sol est essentiel au bon développement des plantes et l'eau stagnante exerce toujours un mauvais effet. Les racines pénètrent si profondément que la gesse des bois est très résistante à la sécheresse.

Valeur agricole: La plante sauvage a un goût amer et les bestiaux n'en sont pas friands. On prétend également qu'elle peut 
occasionner des désordres sérieux. La variété cultivée n'a pas ce goût amer, elle est plus riche en éléments nutritifs et sa valeur nutritive est beaucoup plus grande.

La valeur agricole de la gesse des bois est assez disputée. On prétend que c'est une plante fourragère précieuse surtout en mélange, pour le foin, mais les résultats donnés par les expériences ne nous encouragent pas à en recommander la culture sur une grande échelle. On ne peut la recommander dans les assolements courts.

Fourrage: Cultivée pour son foin la gesse des bois doit être coupée dès que les fleurs commencent à apparaître ou dès que les tiges commencent à verser. Comme la végétation débute de bonne heure au printemps et se maintient toute la saison on peut en tirer deux ou trois coupes dès à partir de la première année.

Production de la graine: Ce sont les sols légers et sablonneux qui donnent les meilleurs résultats. La récolte atteint son développement maximum à la troisième ou à la quatrième année. L'engrangement est difficile, car les gousses mûrissent inégalement et se rompent pendant les chaleurs, éparpillant la semence. On les cueille généralement à la main dès qu'elles sont mûres.

Qualité de la graine: La graine a à peu près la même grosseur et la même forme que celle des vesces; elle est brune, sa surface est rendue rugueuse par un réseau de veines courbes et rameuses.

L'agriculture inspire du courage à l'homme puisque les campagnes qui le nourrissent ne sont point défendues par des forteresses. Elle est, dans tous les gouvernements, la plus honorée des professions, parce qu'elle donne à l'Etat les citoyens les plus vertueux et les mieux intentionnés.-Xénophon, l'Economique, 434-355 A.J.C.

Aux approches du printemps, lorsque la glace descend en eau des monts encore tout blancs de neige et que la neige amollie se détend au souffle du zéphyr, il est temps que le taureau commence à gémir sous le joug de la charrue enfoncée profondément dans le sol et que le soc reluise sous le frottement du sillon. Seule elle ne trompe pas l'espérance du laboureur toujours avide, la terre ensemencée qui aura senti par deux fois le soleil et par deux fois le froid; seule elle remplira ses greniers a les faire craquer sous le poids d'une récoite sans pareille.-Virgile, les Géorgiques traduction d'Henri Lantoine. 


\section{SOJA OU SOYA (Glycine hispida Maxim.)}

Autre nom français: Pois chinois.

Noms anglais: Soy ou Soja bean.

Description botanique: Le soja est annuel; il ressemble à la fève commune des champs mais s'en distingue aisément par les poils plus ou moins nombreux dont il est couvert. Les tiges, rameuses à la base, atteignent une hauteur de un à quatre pieds; elles portent beaucoup de grandes feuilles dont chacune se compose de trois folioles de la même grosseur et de la même forme que celle des fèves ordinaires. Les fleurs qui sont en grappes épaisses sont du type ordinaire des légumineuses. Elles sont blanchâtre à pourpre. Les gousses sont couvertes de poils courts et raides et contiennent généralement de deux à trois graines.

Distribution géographique: Le soja n'est pas connu à l'état sauvage; il est probable qu'il descend de la Glycine Soja Sieb. et Zucc., une espèce étroitement apparentée qui pousse à l'état sauvage dans la Mandchourie, la Chine et la Cochinchine. Sa culture est très ancienne en Chine et au Japon; le grand nombre de variétés produites en ce pays l'indiquent. On le cultive sur une petite échelle depuis une centaine d'années environ dans le sud de l'Europe et il a été introduit tout récemment aux Etats-Unis et au Canada.

Valeur agricole: En Chine et au Japon le soja est employé principalement comme nourriture. On fait rôtir et moudre les fèves pour en faire du pain.

Culture: Le soja préfère les terres riches en matière organique, bien égouttées et non acides. Il faut inoculer au sol les bactéries convenables. Les plantes ne sont pas très sensibles à la sécheresse.

Variétés: Les variétés, qui sont nombreuses, diffèrent l'une de l'autre au point de vue du développement, de la durée de la végétation, de la couleur des fleurs et des graines et de leur adaptation au climat. La Medium Green, une variété productrice d'un grand mérite pour la production du foin ainsi que pour la production de la graine, est la mieux adaptée au Canada.

La plante tire son nom de Soy, un produit que l'on obtient au moyen d'une fermentation longue et compliquée d'un mélange de 
fèves Soy cuites, de blé moulu, de riz ou d'orge passés à la vapeur auxquels on ajoute plus tard de l'eau et du sel. Le Soy est l'élément principal des sauces Worcester et autres sauces à goût piquant.

Fourrage: Le soja ne saurait faire concurrence au trèfle rouge pour la production du foin dans les rotations régulières de la ferme, mais on peut y avoir recours lorsqu'une récolte de printemps a manqué. Coupé à l'époque convenable, le foin est très nourrissant; on fauche au moment où les gousses commencent à se développer. Plus le développement de celles-ci est avancé, plus le foin est coriace et moins il est savoureux et les feuilles, qui constituent la partie la plus nutritive, sont en grande partie perdues. On sème de un et demi à deux boisseaux de graine à l'acre quand on veut faire du foin.

Production de la semence: Bien cultivé le soja donne une production avantageuse de graine. Comme les gousses s'ouvrent quand elles sont tout à fait mûres la coupe tardive cause des pertes, surtout avec la variété Medium Green où les graines s'éparpillent beaucoup. Dans la production de la graine on fauche quand les gousses commencent à jaunir. On sème un boisseau de graine à l'acre.

Qualité de la graine: Dans certaines variétés les graines ont la grosseur et la forme du pois; dans d'autres elles sont deux fois plus larges et dans d'autres encore elles ressemblent à de petites fèves. La couleur est jaune, blanche, verte, brune ou noire. La graine est riche en protéine et en huile et peut être utilisée de la même manière que les autres éléments concentrés qui renferment de l'huile et de la protéine.

\section{NAVETTE (Brassica Napus L.)}

Nom anglais: Rape.

Description botanique: La navette, surtout quand elle est jeune, ressemble à certaines variétés de rutabagas ou navets de Suède. Sa racine n'est pas aussi charnue que celle de ce dernier, elle ressemble plutôt à la racine du chou et s'enfonce dans le sol jusqu'à une profondeur considérable. Les feuilles sont nombreuses, fortes, étalées, d'un vert bleuâtre, douces, succulentes et tendres. 
Les fleurs forment une inflorescence forte, large, jaune brillante et elles ont environ un demi-pouce de large quand elles ont atteint leur plein développement. Elles sont fécondées par les insectes.

Distribution géographique: La navette sauvage est propre au nord de l'Europe où elle se rencontre particulièrement sur le rivage de la mer. On la cultive dans presque toute l'Europe, le nord de l'Asie, les Etats-Unis et l'est du Canada.

Culture: La navette exige un sol riche, bien cultivé et assez frais. Les sols argilo-sablonneux qui contiennent une bonne proportion de matière organique donnent les meilleurs résultats. Les rendements sont généralement faibles sur les sols légers et sablonneux ou sur les argiles lourdes. Elle préfère le climat frais, mais elle vient assez bien dans les régions chaudes et sèches, pourvu que le sol soit assez humide et assez riche.

Variétés: La navette est annuelle ou bisannuelle. On cultive généralement les variétés annuelles pour leur graine; on les appelle 'navette d'été'. La navette d'hiver, telle que la Dwarf Essex, est bisannuelle. Seules les variétés bisannuelles ont de l'importance comme plantes fourragères au Canada.

Végétation: La variété Dwarf Essex et les autres variétés fourragères ressemblent assez aux navets dans leur mode de végétation. On sème la même quantité de graine par acre, de deux à quatre livres, et à peu près en même temps, soit en rangs soit à la volée. Le feuillage est prêt à être pâturé en automne. Si on les protège contre les froids rigoureux de l'hiver, les tiges qui restent donnent de la graine l'année suivante.

Valeur agricole: La navette est très nourrissante pour les moutons, les porcs, les bêtes de rente et d'engrais. Comme elle est très succulente-c'est-à-dire comme elle contient une forte proportion d'eau-il est difficile d'en faire du foin, du reste son foin a relativement peu de valeur car les feuilles s'émiettent en poussière. On s'en sert principalement pour les pâturages, et jusqu'à un certain point comme fourrage vert. Elle est peu employée pour l'ensilage. 



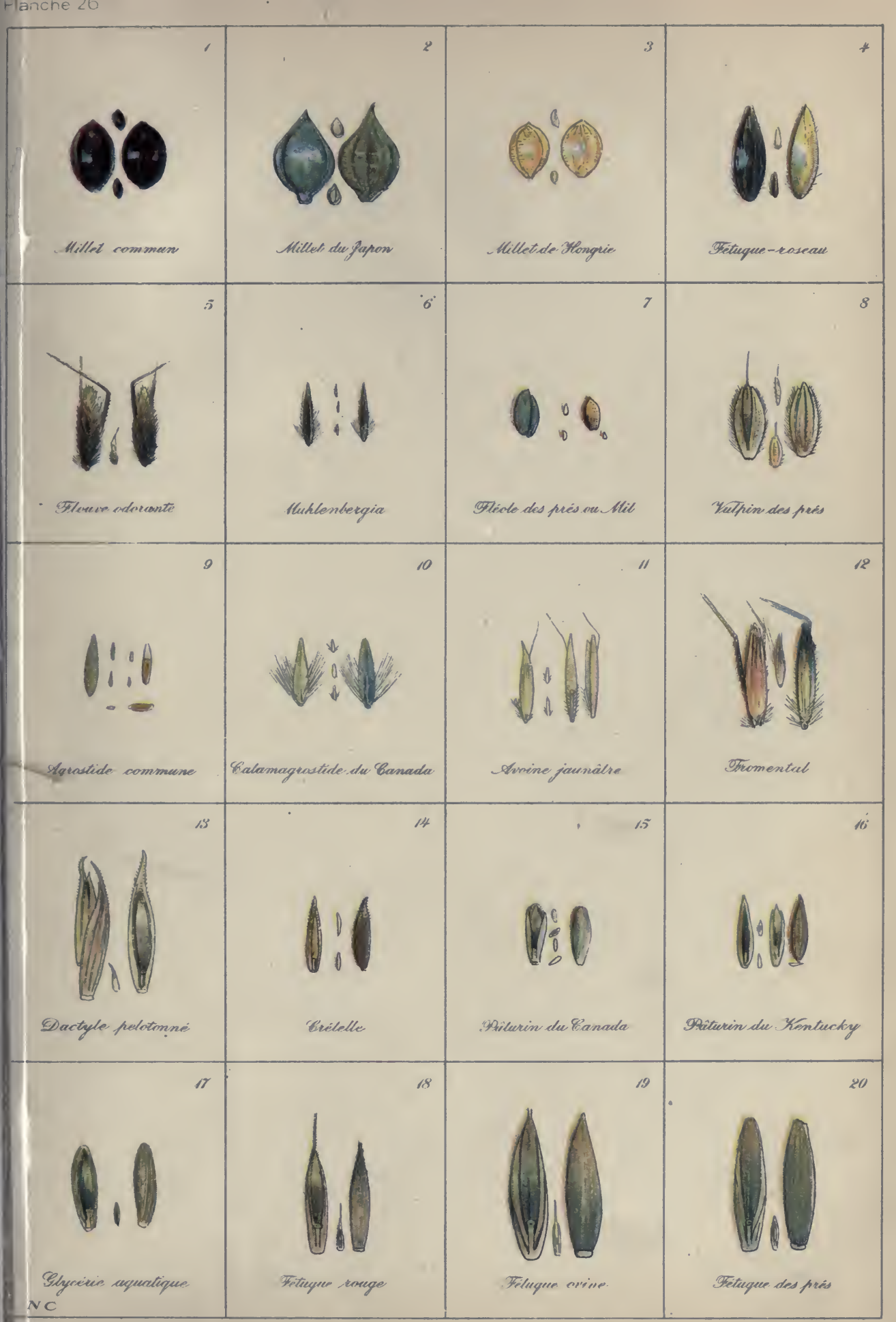





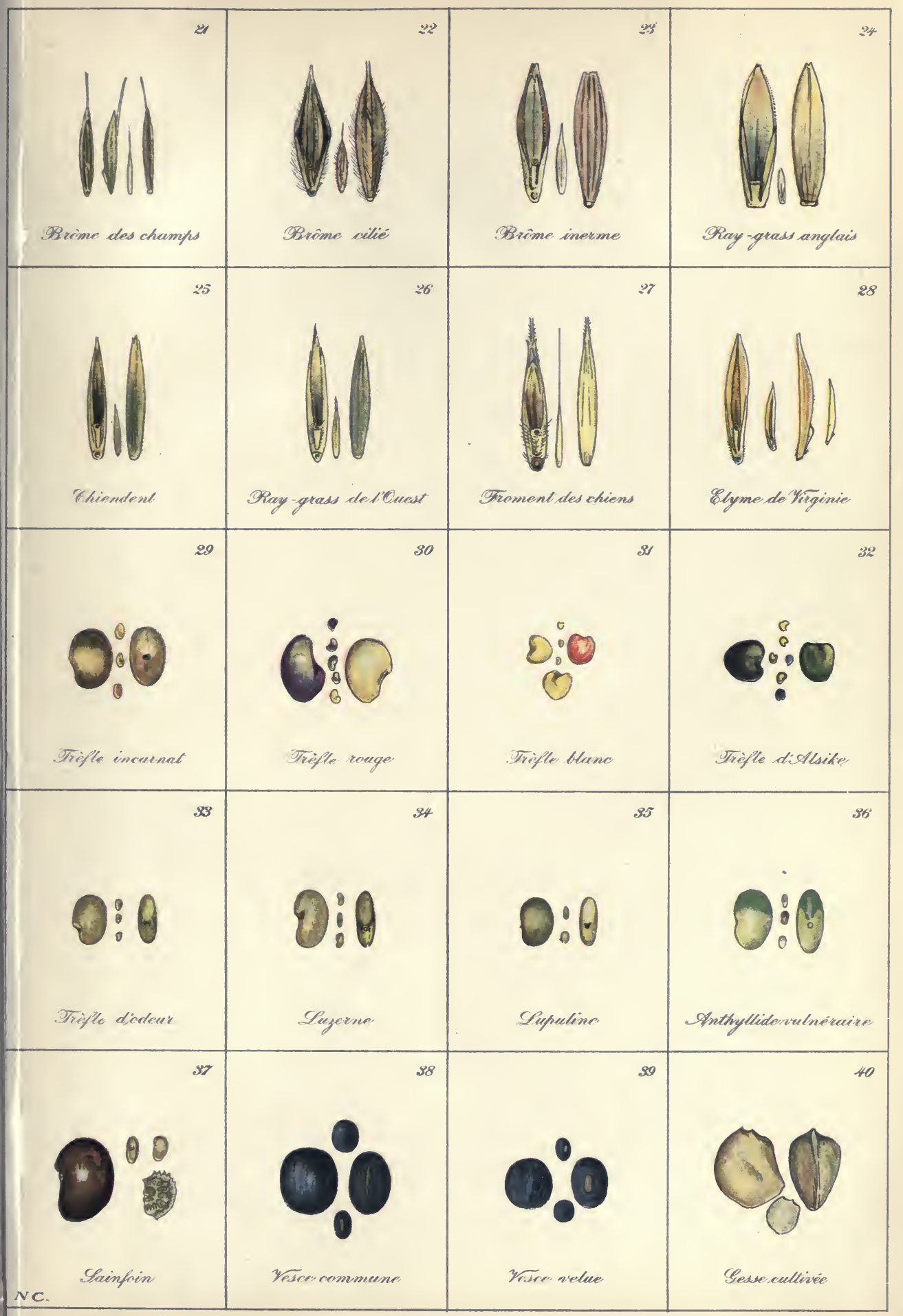





\section{Glossaire des termes techniques.}

Aisselle- Angle que fait la feuille avec la tige ou la branche qui la porte. C'est à l'aisselle des feuilles que naissent ordinairement les bourgeons.

Alterne- $\quad$ Se dit des feuilles ou des fleurs qui croissent des deux côtés de la tige et des branches mais qui ne sont pas en face les unes des autres.

AnNuelle- Qui ne dure qu'un an. Annuelle d'hiver, plante qui germe en automne, fleurit le printemps suivant, produit ses fruits puis meurt.

Auto-FÉconda- La fécondation d'une plante par son propre polTION- len.

AUTO-STÉRILE-Plante qui est incapable de produire des graines à moins d'être fécondée par une autre plante.

BACTÉRIE- Sorte de plantes extrêmement petites qui ne peuvent être vues qu'au moyen du microscope.

BASALE- Qui est lié ou qui appartient à la base.

BASE- L'extrémité d'une feuille ou d'un fruit par laquelle ils sont attachés à leur soutien.

BisAnNuElle - Qui vit deux ans. Une plante bisannuelle met deux saisons à terminer sa croissance. Elle passe la première à rassembler et à emmagasiner de la nourriture et la deuxième à produire des fleurs et des graines, après quoi elle meurt.

Bulbe- Bourgeon écailleux, charnu, qui occupe le bas de la tige; ordinairement souterrain.

CARÈne- Nom donné aux pétales inférieurs des fleurs papilionacées, qui simulent la carène d'un vaisseau, voir page 18.

Composé- Composé de deux ou de plusieurs parties semblables réunies en une seule. Feuille composée, feuille divisée en folioles séparées.

CoMprimé- Aplati.

Cotylédon: Parties charnues de la graine qui, lors de la germination, formeront les premières feuilles. Voir pages 8 et I4.

DÉcombant - Se dit des parties des végétaux, et particulièrement des tiges, qui se dirigent en bas et en dehors, en se couchant à cause de leur faiblesse et de leur flexibilité.

DÉCORTIQUER-Dépouiller de sa première enveloppe, en parlant d'un fruit, d'une graine.

DresséE- Droite.

ETAMine- Organe sexuel mâle des végétaux à fleurs; voir page II. 
ESPÈCE- Une catégorie de plantes qui possèdent les mêmes formes et les mêmes propriétés qu'elles transmettent à leur progéniture.

ETENDARD- Pétale supérieur de la corolle d'une légumineuse dressé et étalé à la façon d'un étendard; voir page I 8.

FÉcondation- Procédé par lequel le pollen rend l'ovaire fertile; production du fruit.

FÉcondation Fécondation d'une plante par le pollen d'une autre CROISÉE- plante.

FEUILLÉ-

FEUILlUQui a des, feuilles.

Foliole-

GenOuIlléQui a beaucoup de feuilles.

GENRE-

Se dit de chaque division du limbe d'une feuille composée, ou encore des sépales et des pétales.

Glabre-

GlauQue-

GlumeCourbé de façon à former un angle.

Un groupement d'espèces qui possèdent certaines caractéristiques communes.

Groupe-

Lisse, sans poil.

D'un vert marin ou d'une couleur bleu grisâtre.

Une des bractées verdâtres qui enveloppent extérieurement un épillet de graminée. Voir page II.

Groupe de fleurs dans lequel un nombre de fleurs à queues courtes et égales sont disposées sur un axe commun et délié comme dans la groseille rouge ou blanche.

Hybride- Un animal ou une plante qui provient du croisement de deux espèces différentes.

INDIGÈNE- Propre à un pays ou à un district.

INFLORESCENCE-Mode de groupement des fleurs d'une plante ou ensemble des fleurs ainsi groupées.

INOCULER- Fournir au sol certaines sortes de bactéries.

LATÉral- Venant de côté.

LEGUME-

$\begin{array}{ll}\text { Lemma- } & \text { Glumelle infér } \\ \text { Ligule - } & \text { Voir page Io. }\end{array}$ Une gousse dans laquelle les graines sont attachées d'un côté seulement.

LIMBE-

Nervure-

NERVURE MÉDIANENoDosite-

NoEUD-

OvaIRE-

Palea-

Panicule-

Partie principale, élargie et étalée de la feuille.

Faisceaux de fibres vasculaires formant la charpente des feuilles.

Pétale-

La nervure centrale ou principale d'une feuille.

Renflement qui se forme sur les racines de certaines plantes, surtout des légumineuses.

La jointure d'une tige ou la partie où se trouvent attachées une feuille ou plusieurs feuilles.

Partie du pistil dans laquelle se forment les graines. Glumelle supérieure des graminées. Voir page II.

Groupe de fleurs, à tige principale, portant des rameaux eux-mêmes ramifiés mais moins longs.

Nom de chacune des pièces foliacées de la corolle. 
Prstil- Organe femelle de la fleur composé de l'ovaire, du style et du stigmate; voir page 18 .

Pollen- Poussière fécondante contenue dans les sacs de l'anthère.

RAMPANT- Courant à la surface ou près de la surface du sol, et souterraine.

RACINE PIVo- Racine généralement forte et profonde par laquelle TANTE- la tige principale se prolonge dans la terre.

REJETON- Nouvelle pousse produite par un végétal, dans le voisinage immédiat du pied de la tige ou du collet de la racine.

Rhizome- Tige souterraine oblique ou horizontale, dont les bourgeons s'élèvent au dehors en tiges aériennes.

SÉPale- Nom scientifique des folioles du calice.

Souche- Partie souterraine de la tige des plantes vivaces, souvent synonyme de rhizome.

Spathe- Grande bractée membraneuse qui enveloppe les fleurs.

Stabulation- Séjour et entretien temporaire ou permanent des animaux dans une étable.

STÉRILE-

Stigmate-

STIPUle- $\quad$ Nom des petites dépendances de la feu

Sans fruit, incapable de produire des semences. Sommet glanduleux de l'ovaire ou du style.

Stolon- Rejet rampant sur terre, ou sous terre produisant des racines ou de nouvelles tiges.

Tige Ascen- Celle qui se dresse vers le ciel après s'être dirigée DANTE- horizontalement, comme dans la véronique en épis, le trèfle des prés, etc.

TRIFOLIÉ- Ayant trois folioles.

TUBERCULE- Renflement cellulaire que présente la partie souterraine de certaines plantes.

VivacePlantes vivaces, plantes qui vivent plusieurs années.

VRILleSorte de filament qui croft sur certaines plantes et qui s'enroule en spirale sur les corps voisins. 



\section{INDEX.}

PAGE

PAGE

Agropyre à barbe............... 102

Agropyre de l'ouest.............. I0I

Agropyron occidentale............ ror

Agropyron repens............... 102

Agropyron Richardsonii........... 102

Agropyron Smithii.............. 101

Agropyron tenerum.............. 98

Agrostide commune............. 57

Agrostide stolonifère............ 55

Agrostide traçante............... 55

Agrostis alba................. 55

Agrostis stolonifera ............. 55

Agrostis vulgaris.............. 57

Alfalfa..................... 125

Alkali grass.................. I0I

Alopecurus pratensis............. 53

Alpiste des canaries.............. 46

Alsike clover.................. I 19

Anthoxanthum odoratum........... 47

Anthyllide vulnéraire............. I39

Anthyllis vulneraria............ I39

Arrhénantère............... 62

Arrhenatherum elatius............ 62

Austrian brome grass............ 90

Austrian brome hay............. 90

Avenat.................... 62

Avoine élevée ou fromental........ 62

jaunâtre.................. 60

Awned wheat grass.............. 102

Awnless brome grass............. 90

Bald rye grass................ 103

Bald wheat grass............... 98

Barnyard grass................. 45

Barnyard millet................ 45

Blé d'Espagne................. 34

Blé de Turquie............... 34

Blé d'Inde.................. 34

à gousse........................ 36

coché................ 37

mou................. 37

soufflé................ 37

sucré................. 37

sucré féculeux.............. $3^{8}$

vitreux.............. 37

Bird grass.................. 70

Black Medick................ 137

Blue grass.................. 70

Blue joint................. ror

Blue joint grass................ 58

Bokhara clover................ 123

Brassica Napus................. 150

Bromus arvensis.............. 92

Bromus ciliatus................ 93

Brome cilié................. 93

Brome des champs.............. 92

Brome grass.................. 90

Brome inerme................... 90

Bromus inermis............... 90

Bujoline.................. 137

Calamagrostide du Canada......... 58

Calamagrostis canadensis..................... $5^{8}$

Canada bent-grass................ $5^{8}$

Canada blue grass............... 68

Canadian blue grass.............. 68

Chiendent.................. 102

Chiendent queue de renard......... 53

Cocksfoot.................... 64

Colorado blue stem.............. I0I

Common Darnel............... 94

Common meadow grass.......... 70

Common millet................ 41

Common vetch................. I42

Couch grass................... I02

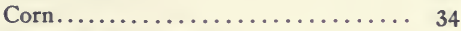

Creeping bent-grass............ 55

Crimson clover.................. I05

Creeping fescue................. 79

Creeping Poa.................. 68

Crested dog's tail................ 66

Crételle....................... 66

Cuscuta epithymum........... 132

Cuscuta racemosa................ I14

Cynosurus cristatus.............. 66

Dactyle pelotonné............. 64

Dactylis glomerata............. 64

Deyeuxia canadensis.............. $5^{8}$ 
Dog's tail grass................ 66

Dutch clover.................. II6

Echinochloa Crus-galli........... 45

Elyme de Virginie............... I03

Elymus virginicus............... I03

English blue.grass.............. 68

English grass................. 70

English Rye or Ray grass........... 94

Epillets de graminées.............. I I

Esparsette commune............. I40

Esparcette................... 140

Etablissement des prairies et pâturages....................... 2 I

Evergreen grass............... 85

Faba vulgaris................ I45

False oat grass............... 62

False red top................... 76

Faux froment................. 62

Faux seigle................. 62

Fécondation des graminées.......... II

Fécondation des légumineuses........ I 8

Fenasse pain-vin............... 62

Festuca arundinacea.............. 88

Festuca elatior................. 85

Festuca heterophylla............. 84

Festuca ovina................. 8 8 I

Festuca ovina, var. duriuscula....... 83

Festuca ovina, var. tenuifolia....... 83

Festuca pratensis............... 85

Festuca rubra................. 79

Fétuque d̀ feuilles diverses.......... 84

Fétuque des prés............... 85

Fétuque durette................ 83

Fétuque élevée............... $85^{-8}$

Fétuque ovine............. $8 \mathrm{I}$

Fétuque ovine à feuilles fines........ 83

Fétuque roseau................. 88

Fétuque rouge................. 79

Feuilles des graminées............ I0

Feuilles des légumineuses.......... I6

Fève à cheval................. I45

Féverole.................... 145

Fine leaved Sheep's Fescue......... 83

Fiorin grass................. 55

Flat pea................... 147

Fléole des prés............... 49

Fleurs des légumineuses............ I7
Flouve odorante............... 47

Fowl meadow grass............. 76

Foxtail millet................. 43

Fromental.................... 62

Fruit des graminées............. 12

Fruit des légumineuses........... I9

German clover................ 105

Germination des graminées......... 8

Germination des légumineuses....... 15

Gesse cultivée................... 146

Gesse des bois................. 147

Glyceria aquatica................ 77

Glycérie aquatique............. 77

Glycine hispida............... I49

Golden oat grass.............. 60

Graine des graminées............. 8

Graine des légumineuses........... I4

Graminées.................... 8

Grand trèfle rouge.............. II5

Grass pea................... 146

Green grass.................... 70

Gros millet.................. 34

Hairy vetch.................. 144

Hard fescue................. 83

Herd's grass.................. 49

Horse bean................. 145

Hungarian brome grass........... 90

Hungarian fodder grass.......... 90

Inflorescence des graminées......... I0

Inflorescence des légumineuses....... 17

Indian corn .................. 34

Italian clover................. 105

Ivraie vivace................ 94

Ixophorus itálicus............. 43

June grass................... 70

Kentucky blue grass............. 70

Kidney vetch................. 139

Knot root grass............... 48

Lathyrus sativus............. 146

Lathyrus silvestris............. 147

Légumineuses................. 14

Lentille d'Espagne............. 146

Lentille Suisse................ 146

7 Lolium multiflorum.............. 96 
Lolium perenne................ 94

Lucerne.................... I25

Lupuline................... 137

Luzerne commune............... 125

Luzerne cultivée................ I25

Luzerne faucille............... I33

Luzerne houblonnée.............. 137

Luzerne moyenne................ 134

Luzerne rustique.............. I34

Maiss.

Maize......................... 34

Mauvaises herbes, leur effet......... 30

Meadow cat's tail............... 49

Meadow fescue................. 85

Meadow foxtail............... 53

Meadow oat grass............. $\mathbf{6}_{2}$

Medicago falcata.............. I33

Medicago falcata $x$ sativa.......... I34

Medicago lupulina............... 137

Medicago media................ I 134

Medicago sativa................ 125

Medicago silvestris............. I34

Melilotus alba................. I23

Mélilot blanc.................. 123

Mignonette.................. I37

Mil....................... 49

Millets...................... 40

à grappe............... 43

commun............... $4 \mathrm{I}$

des oiseaux.............. 43

d'Italie................. 43

long.................... 46

patte de coq.............. 45

perlé.................... 44

Minette.................. 137

Muhlenberge.................. 48

Muhlenbergia glomerata.......... 48

Muhlenbergia racemosa......... 48

Navette................... ${ }^{150}$

Oat grass................... 62

Oplismenus Crus-galli.............. 45

Onobrychis sativa.............. I40

Orchard grass.................. 64

Panic d'Italie.................. 43

Panicum Crus-galli.............. 45

Panicum miliaceum............... 41
Pâturages.................... 2I

Pâturin bleu du Canada............. 68

bleu du Kentucky.......... 70

commun................ 73

comprimé.............. 68

des bois.............. 75

des marais.............. 76

des prés................ 70

Pearl millet.................. 44

Petit trèfle rouge............... I07

Pennisetum typhoideum........... 44

Perennial rye grass............... 94

Phalaris arundinacea............. 46

Phleum pratense............... 49

Plantes-abris................. 22

Plantes légumineuses.............. I4

Poa compressa................. 68

Poa flava..................... 76

Poa nemoralis............... 75

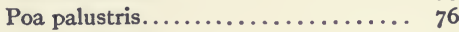

Poa pratensis.................. 70

Poa serotina................... 76

Poa triflora................... 76

Poa trivialis................... 73

Pois breton................... 146

Pois carré.................... 146

Pois chinois................ 149

Pois de brebis................ 146

Pois gras.................... 146

Prairie, développement............ 26 protection en hiver.......... 27

Racines des graminées............ 9

Racines des légumineuses.......... I5

Randall grass................. 85

Rape....................... 150

Ray-grass anglais.............. 94

Ray-grass de France............ 62

Ray-grass de l'Ouest............. 98

Ray-grass d'Italie.............. 96

Ray-grass vivace............... 94

Red clover................... I07

Red fescue.................. 79

Red top................... 55

Reed canary grass.............. 46

Reed fescue................. 88

Rough stalked meadow grass........ 73

Sainfoin..................... 140 
Sand grass.

Sand lucerne.................. I34

Scarlet clover................. ro5

Schedonurus inermis............. 90

Setaria italica................. 43

Sheep's fescue................. $8 \mathrm{r}$

Smaller blue grass............... 68

Slender wheat grass............... 98

Small reed grass........, . . . . . . $5^{8}$

Smooth brome grass.............. 90

Smooth stalked meadow grass........ 70

Soja........................ 149

Soy ou Soja bean................ 149

Soya......................... 149

Spear grass................... 70

Spring vetch................ I42

Sweet vernal grass............... 47

Tall fescue....................85-88

Tall meadow oat grass............ 62

Tall oat grass................. 62

Tare........................ $\mathbf{1 4 2}^{2}$

Terrel grass.................... I03

Tiges des graminées............. 9

Tiges des légumineuses............ I5

Timothy..................... 49

Trainasse..................... 55

Trèfle blanc..................... I16

Trèfle d'Alsike................... I I9

Trèfle de Roussillon............... I05

Trètle des prés................. I07

Trètle d'odeur.................. I23

Trètle farouche................. I05

Trèfle hybride.................. II9

Trètle incarnat................ I05

Trèfle jaune................. 137

Trètle jaune des sables........... 139

Trèfle Mammouth............... II5

Trèfle noir................... 137

Trè̀le rouge................ I07

Trèfle rouge ordinaire............ 107

Trèfle Zigzag.................. 108

Trifolium hybridum............ 119

Trifolium incarnatum............. 105

Trifolium medium............... 115

Trifolium pratense.............. 107

Trifolium pratense var. perenne...... I08

Trifolium repens................ 116

Trisetum flavescens.............. 60

Valeur agricole des graminées........ I2

Valeur agricole des légumineuses..... 19

Variegated alfalfa............... I34

Various-leaved fescue............ 84

Vesce commune............... 142

Vesce de Russie................ 144

Vesce velue................... 144

Vicia sativa................. 142

Vicia villosa.................. I44

Virginia blue grass............... 68

Virginia Lyme grass............. I0 3

Vulnéraire.................... I39

Vulpin des prés............... 53

Water meadow grass. . . . . . . 77

Western rye grass................ 98

Western wheat grass............. I01

Wheat grass.................. 10 3

White Bent-grass............. 55

White clover................... I 6

White sweet clover............. I23

Winter vetch................ I44

Wire grass................... 68

Wood meadow grass............. 75

Yellow false oats............... 60

Yellow lucerne................ 133

Yellow oat grass............... 60

Yellow trefoil............... 137

Zea Mays................... 34

Zigzag clover.............. 108 
Date Due

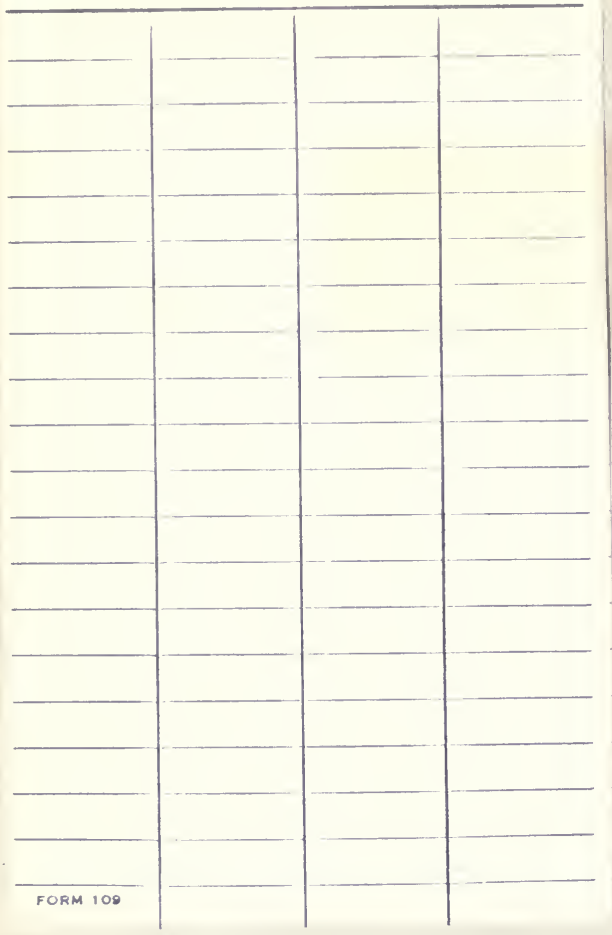




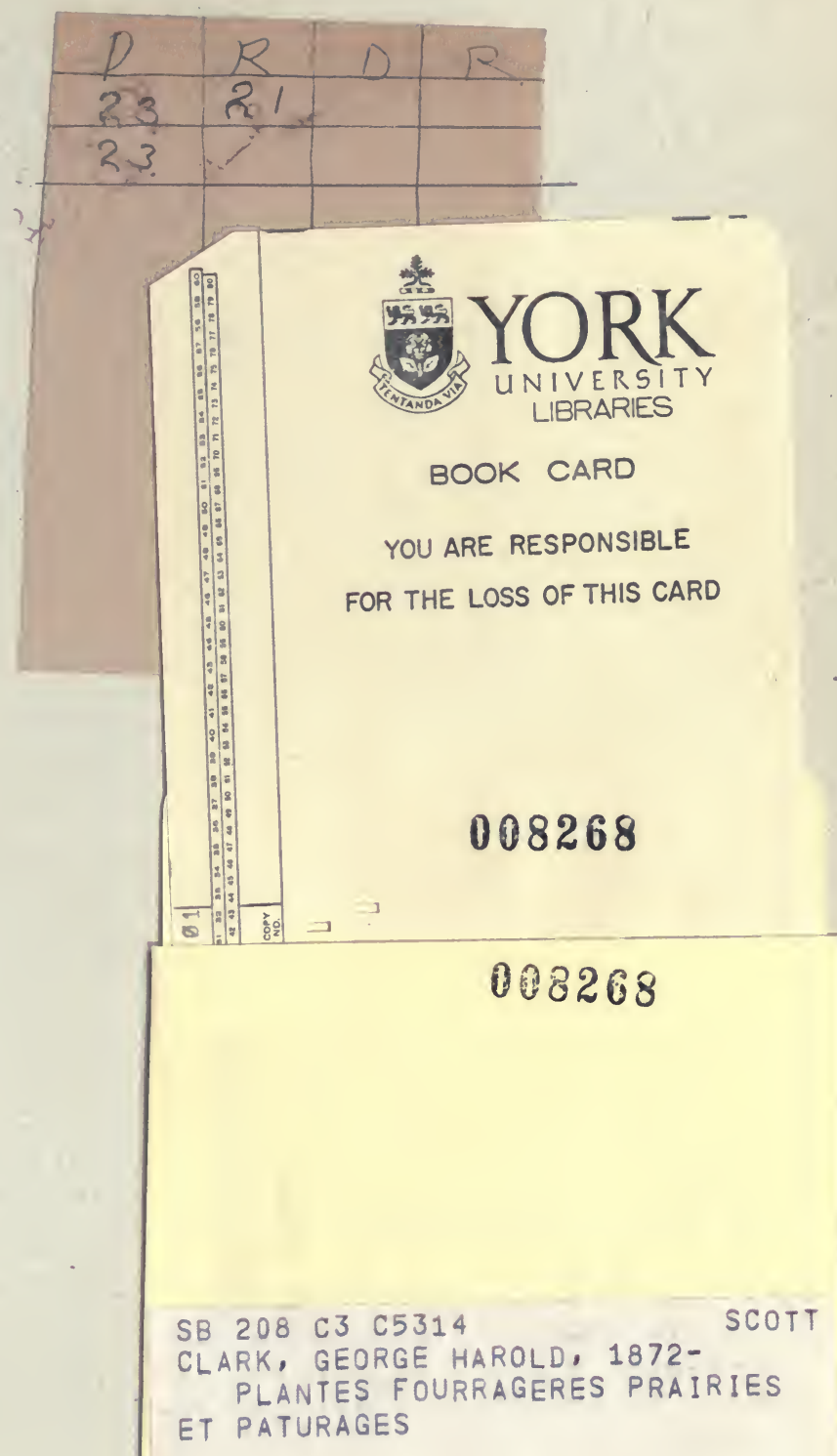


